\title{
Glutamine metabolism : effects of disease and glutamine supplementation
}

Citation for published version (APA):

Hulsewé, K. W. E. (2007). Glutamine metabolism : effects of disease and glutamine supplementation. [Doctoral Thesis, Maastricht University]. Universiteit Maastricht. https://doi.org/10.26481/dis.20070608kh

Document status and date:

Published: 01/01/2007

DOI:

10.26481/dis.20070608kh

Document Version:

Publisher's PDF, also known as Version of record

\section{Please check the document version of this publication:}

- A submitted manuscript is the version of the article upon submission and before peer-review. There can be important differences between the submitted version and the official published version of record.

People interested in the research are advised to contact the author for the final version of the publication, or visit the DOI to the publisher's website.

- The final author version and the galley proof are versions of the publication after peer review.

- The final published version features the final layout of the paper including the volume, issue and page numbers.

Link to publication

\footnotetext{
General rights rights.

- You may freely distribute the URL identifying the publication in the public portal. please follow below link for the End User Agreement:

www.umlib.nl/taverne-license

Take down policy

If you believe that this document breaches copyright please contact us at:

repository@maastrichtuniversity.nl

providing details and we will investigate your claim.
}

Copyright and moral rights for the publications made accessible in the public portal are retained by the authors and/or other copyright owners and it is a condition of accessing publications that users recognise and abide by the legal requirements associated with these

- Users may download and print one copy of any publication from the public portal for the purpose of private study or research.

- You may not further distribute the material or use it for any profit-making activity or commercial gain

If the publication is distributed under the terms of Article $25 \mathrm{fa}$ of the Dutch Copyright Act, indicated by the "Taverne" license above, 


\section{GLUTAMINE METABOLISM: Effects of Disease and Glutamine Supplementation}

Karel Willem Ewoud Hulsewé 


\title{
GLUTAMINE METABOLISM: Effects of Disease and Glutamine Supplementation
}

\author{
PROEFSCHRIFT \\ ter verkrijging van de graad van doctor \\ aan de Universiteit Maastricht, \\ op gezag van de Rector Magnificus, \\ Prof. mr.G.P.M.F. Mols \\ volgens het besluit van het College van Decanen, \\ in het openbaar te verdedigen op \\ vrijdag 8 juni 2007 om 12.00 uur
}

door

Karel Willem Ewoud Hulsewé

5 Juni 1968, Rheden 


\section{Promotores}

Prof. dr. P.B. Soeters

Prof. dr. M.E. von Meyenfeldt

\section{Beoordelingscommissie}

Prof. dr. R.W. Stockbrügger, voorzitter

Prof. dr. M.J.H.M. van Dieijen - Visser

Prof. dr. P.J.E.H.M. Kitslaar

Prof. dr. P.A.M. van Leeuwen, VU medisch centrum Amsterdam

Prof. dr. K.M.L. Leunissen 


\section{Vormgeving en Layout:}

D\&L graphics

\section{Illustraties:}

John Derwall en Silvie Meijers, D\&L graphics

www.dlgraphics.nl

info@dlgraphics.nl

\section{Druk:}

Schrijen-Lippertz-Huntjens

ISBN-10: 90-8590-018-2

ISBN-13: 978-90-8590-018-4

The studies presented in this thesis were performed at the Nutrition and Toxicology Research Institute Maastricht (NUTRIM).

The research described by this thesis was supported by grants from the Nederlandse Organisatie voor Wetenschappelijk Onderzoek (NWO) and Pharmacia \& Upjohn. 


\section{CONTENTS}

\section{Introduction}

Chapter 1. Introduction 9

II. Effects of trauma and sepsis on glutamine and glutathione metabolism

Chapter 2. Glutamine appearance rate in plasma is not increased after gastrointestinal surgery in humans

Chapter 3. Effects of surgical trauma on interorgan glutamine and amino acid metabolism and organ protein kinetics

Chapter 4. Surgical trauma decreases glutathione synthesis rates in the porcine gut

Chapter 5. Pulmonary glutamine production: effects of sepsis and pulmonary infiltrates

Chapter 6. Inflammation rather than depletion affects glutamine concentrations and intestinal permeability

III. Effects of glutamine supplementation on glutamine and glutathione metabolism

Chapter 7. Does glutamine-enriched parenteral nutrition really affect intestinal morphology and gut permeability?

Chapter 8. Response of glutamine metabolism to glutamine supplemented parenteral nutrition

Chapter 9. Effects of glutamine enriched total parenteral nutrition on 139 interorgan amino acid and gut mucosal glutathione metabolism

IV. Discussion

Chapter 10. Review of glutamine interorgan metabolism in health, 

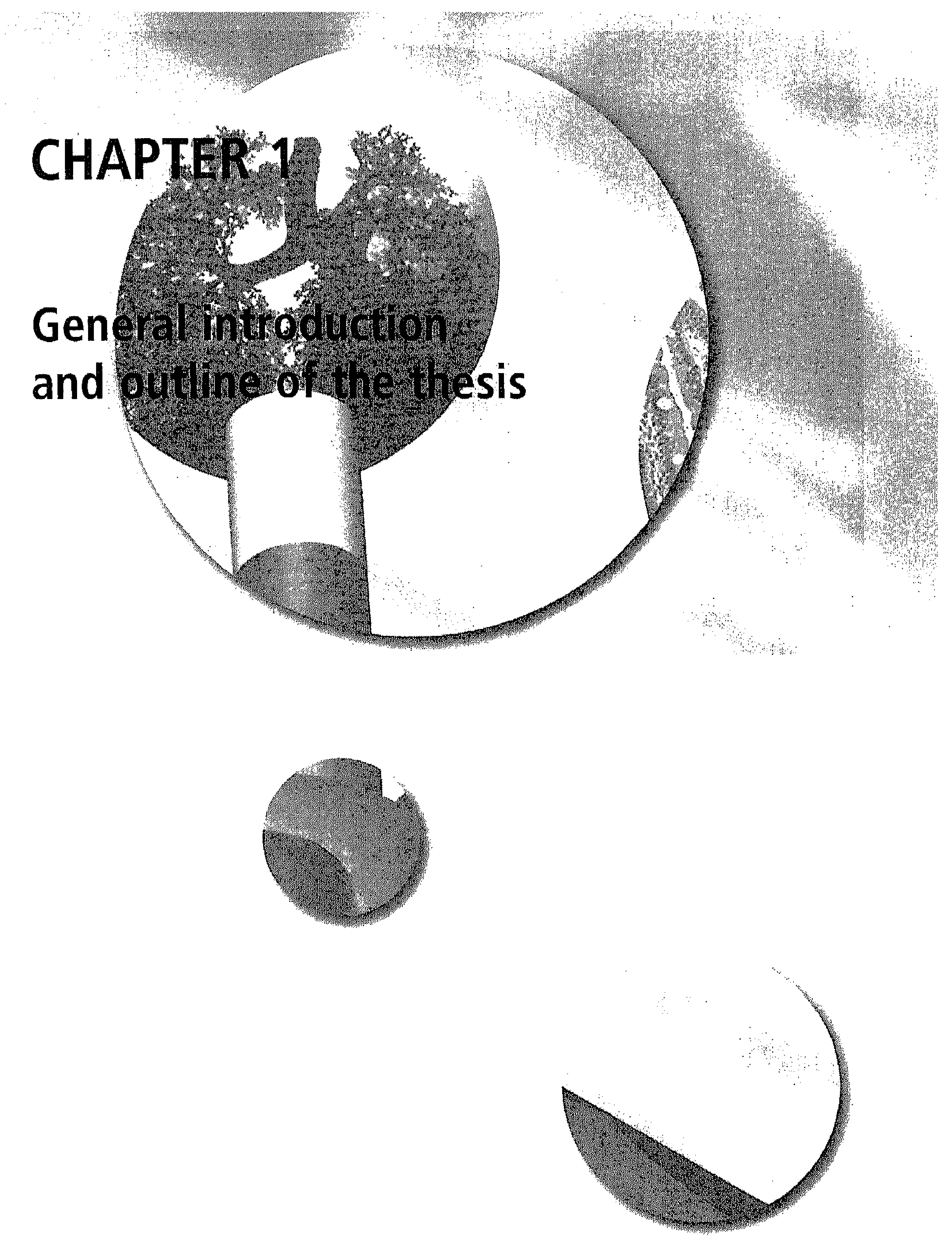


\section{INTRODUCTION}

Following injury, profound changes in metabolism occur in order to support defensive and reparative processes. Changes occur simultaneously in energy, protein, carbohydrate and lipid metabolism, and are orchestrated by a complex system of interacting neurological, endocrine and locally active mediators. Some of the characteristic changes in intermediary metabolism are summarized in table 1.

Table $1 \quad$ Metabolic responses to injury

\section{Energy metabolism}

Basal metabolic rate $\uparrow$

Protein metabolism

Urinary nitrogen loss $\uparrow$

Negative nitrogen balance

Whole body protein turnover $\uparrow$

Nitrogen transfer from muscle to visceral tissues

Acute phase protein synthesis $\uparrow$

Carbohydrate metabolism

Gluconeogenesis $\uparrow$

Glycogenolysis $\uparrow$

Insulin resistance $\uparrow$

Lipid metabolism

Fat oxidation $\uparrow$

Lipolysis $\uparrow$

De novo fatty acid synthesis $\uparrow$

Almost a century ago Whipple contributed significantly to the foundation of current knowledge regarding the effects of injury on nitrogen metabolism! He described the rapid breakdown of protein mass after trauma and (infectious) disease. In addition, he demonstrated that after substantial loss of protein mass due to injury or protein deficient diets, experimentally animals could not adequately respond to injury anymore. Moreover, he described that in dogs different proteins had, after ingestion, varying effects on the resynthesis of proteins. He defined the ability of a food protein, after its ingestion, digestion and absorption to be re-utilized for protein synthesis as the biological value of food protein 2 .

In the 1940-ies Cuthbertson further investigated the catabolic response to injury in humans. He particularly studied patients with femoral fractures. He found that irreversible protein breakdown (measured as urinary nitrogen excretion) not only started immediately after injury, but also that it was rather protracted. Additionally, he demonstrated that protein supplementation was not successful and resulted in increased excretion of nitrogen. 
Later Sir David Cuthbertson identified muscle tissue as the prime source of the increased nitrogen losses he observed in the post-injury state (Cuthbertson, 1979). In the 1980'ies Clowes et al. demonstrated in patients, that muscle is a major source of amino acids which are taken up primarily by the splanchnic bed, in particular the liver ${ }^{2}$. Moreover, he showed that the ability of the splanchnic bed to take up amino acids was inversely correlated to mortality.4.

Another important observation was made by Studley in 1936. In a series of 50 consecutive patients undergoing elective surgery for peptic ulcer disease he found that a preoperative weight loss of over $20 \%$ was associated with an operative mortality of $33 \%$, while mortality in patients who had suffered less than $20 \%$ weight loss amounted to $3.5 \%$. Other factors such as age, impaired cardiopulmonary function, type of surgery, duration of the surgical procedure and the surgeon performing the operation were not associated with outcome. From his observations he found "reason to believe that more patients will be saved, provided efforts are concentrated on the preoperative preparation of those who have lost a good deal of weight"5. Almost 60 years later Giner et al. confirmed the relation between nutritional depletion and surgical trauma 6 .

Depletion is associated with changes in body composition. Depending on the causes decrements in lean body mass and fat mass are found. Usually both compartments are affected. It is very important, however, to realise that in contrast to glucose and fat, no inert protein stores exist. This implicates that loss of protein mass (i.e. lean body mass) leads to organ function even . Indeed it has been observed that nutritional depletion causes loss of in fasted subjects, in whom nofructural changes can be detected. In malnourished but also force frequency characteristics and recovery from composition could be found, changes in are observed 7 . Similarly, functions of other from fatigue by the adductor pollicis muscle and the immune system function ore other organs such as respiratory muscle, heart, gut exact underlying mechanisms have not beemised as a consequence of depletion 8.11 . The

The most important hypothesis underlying the research in this thesis is that depleted patients who become ill or traumatized are incapable to produce sufficient substrate that is obviously of benefit to depleted patients, but doubt has the trauma. Nutritional support is of the nutritional regimen should resemble ordinary raised whether the composition adaptations further improve outcome. A breathrinary daily food or whether specific come from expanding knowledge regarding methough in our thinking in this regard has last decades. In depleted humans not subjected to stress in disease or after trauma in the body protein loss is low, because the amino acids derived turnover is low, and whole almost completely re-utilized for protein synthesis. In the protein breakdown are turnover is increased, but a substantial proportion protein degradation are irreversibly broken pattern produced and consequently also down to yield other substrates. The amino acid crucially different compared to the starving not by the stressed organism is therefore example of an amino acid produced during diset stressed organism. The most striking glutamine. A more detailed hypothesis is therease in excess of its presence in protein is 
lower protein mass cannot produce sufficient quantities of glutamine ${ }^{12}$ and that supplementation of the diet with glutamine might benefit depleted and diseased individuals. This hypothesis underlies part of the research described in the second part of this thesis.

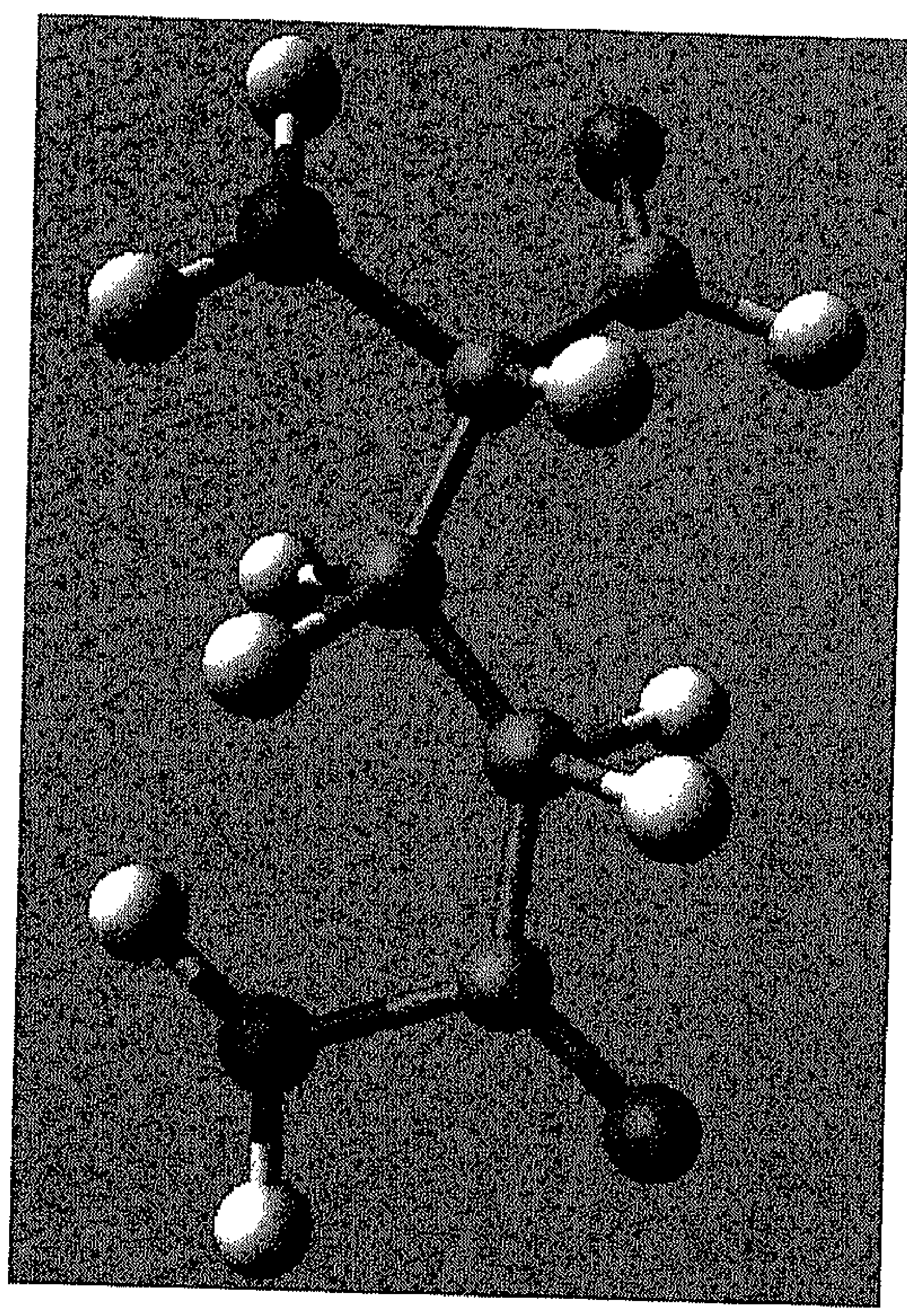

Figure 1 Chemical structure of glutamine

Glutamine constitutes the largest free amino acid pool in the body. Its chemical structure is depicted in figure 1. After a catabolic insult rapid and profound changes occur in plasma and intracellular glutamine concentrations. Decreases of up to $85 \%$ of the free muscle glutamine pool have been described ${ }^{13}$. Glutamine is not an inert metabolite but exerts a multitude of functions. Among others, intracellular glutamine provides substrate for oxidation to yield energy, for synthesis of proteins, but also for synthesis of purines, pyrimidines and glutathione which are essential for DNA and RNA synthesis and anti-oxidant defenses respectively. Through osmotic regulation of cell volume and phosphorylation of proteins glutamine affects protein metabolism ${ }^{14}$. Moreover, glutamine is a major gluconeogenic precursor. As a substrate for ammoniagenesis, glutamine also is involved in acid-base regulation. Finally, glutamine plays an important role in interorgan metabolism. It is a vehicle for transport of carbon skeletons, but
glutamine plays a central role in interorgan megen transported through plasma. Therefore, glutamine plays a central role in interorgan metabolism.

Although glutamine (interorgan) metabolism has been reasonably well characterized in healthy conditions, many gaps remain in our understanding of the effects of trauma or disease. Moreover, only limited data are available describing the consequences of glutamine supplementation on endogenous glutamine metabolism. This thesis intents to fill some of these gaps. In figure 2, the focuses of the studies presented in this thesis are indicated.

Glutamine is produced endogenously in large amounts and has therefore been classified as a non essential amino acid. Glutamine is produced mainly by muscle tissues but under stress the liver and lung also can contribute to glutamine production. The profound changes in glutamine metabolism during catabolic stress on the one hand and positive effects of led to the upplementation in some studies involving catabolic patients on the other, have led to the use of the term conditionally essential for glutamine ${ }^{15}$. If this is true the next 
step is to verify under which conditions endogenous glutamine production becomes deficient. and to determine the optimal dose for glutamine supplementation.

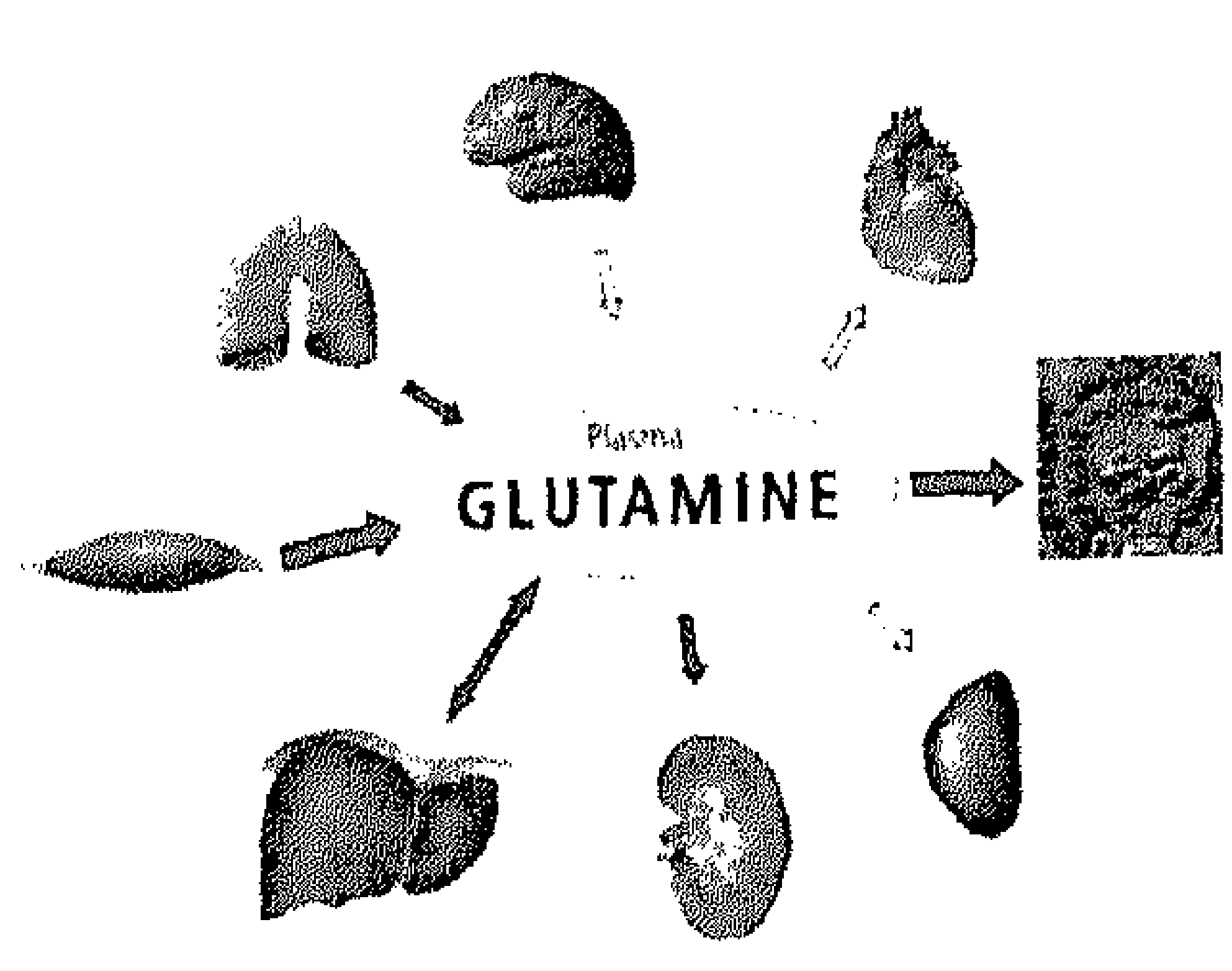

After trauma or during disease

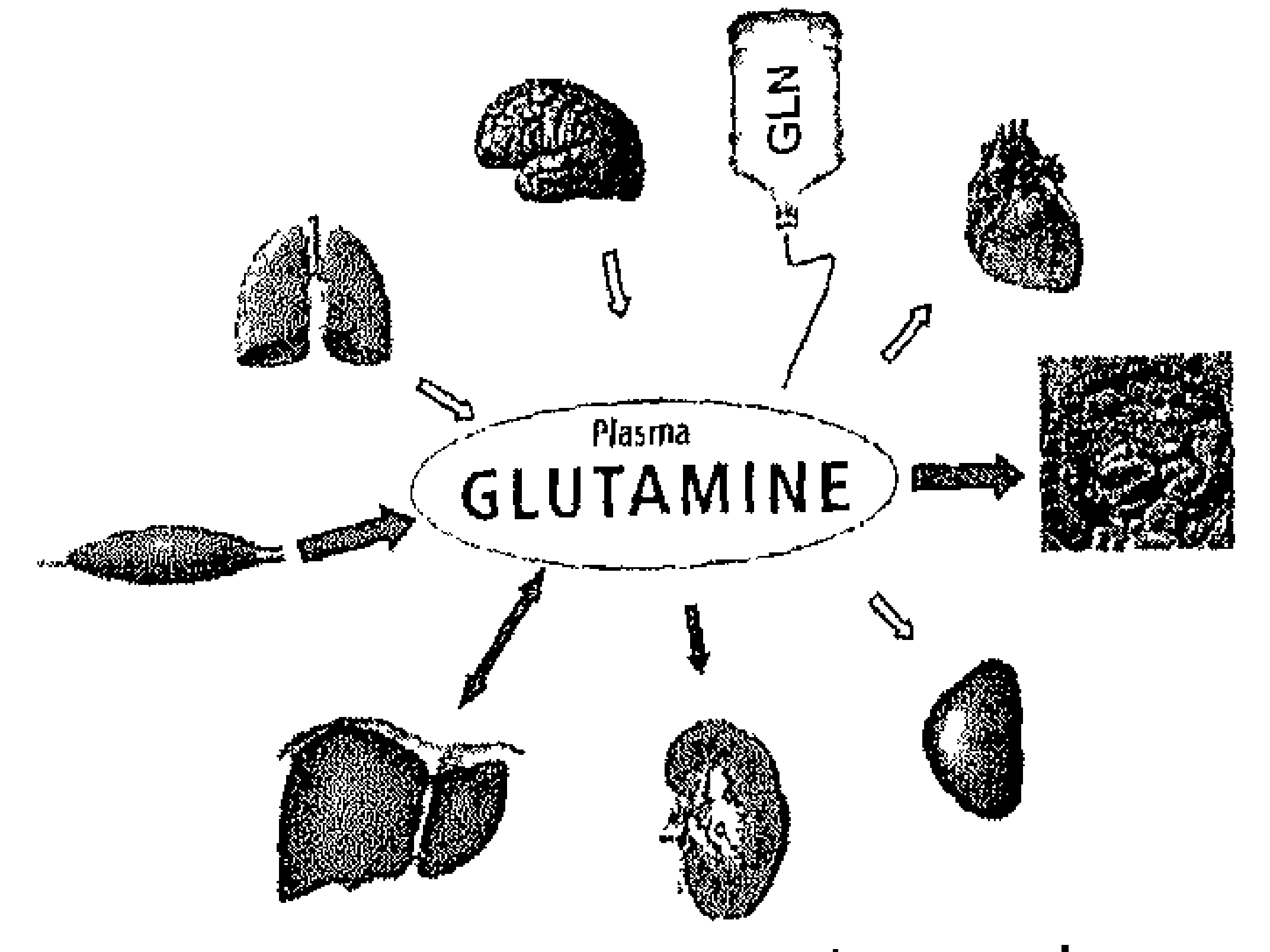

During glutamine supplementation

Figure 2 shematic representation of the contributions of different organs to glutamine interorgan met abolism. The direction of the arrows indicate the net release (arrow towards plasma pool) an mer uptake from the plasma pool larrow directed towards organ). The solid arrows indicate the processes studied in this thesis, whereas the open arrows indicate glutamine fluxes across orath not described in the subsequent chapters. GLN indicates glutamine supplementation.

One of the simplest ways to establish efficacy and simultaneously safety ranges of drugs or food components is to establish dose-response curves. This is problematic because glutamine is produced endogenously by the organism, and the production rates vary according to the circumstances. Consequently it is nearly impossible to provide safe and optimal dose recommendations for all relevant clinical conditions. Alternatively, it has been hypothesized that glutamine can have beneficial effects in those conditions during which the body is unable to furnish adequate amounts of glutamine. Starting with the concept of glutamine as an conditionally essential amino acid, glutamine dosage depends on the difference between a normal endogenous glutamine production and the insufficient production rate in the specific patient. By studying glutamine production in patients in a variety of conditions normal responses in glutarnine metabolism can be defined for relevant clinical conditions without the risk of any possible adverse effects of (high dosed) glutamine administration.

Furthermore, insight in the effects of glutamine supplementation on glutamine endogenous metabolism is essential in order to detect possible adverse effects, to further define indications and estimate glutamine needs. The fact that efficacy of glutamine administration has led to equivocal results without clear explications, underscores this point.

If the optimal amount of glutamine to be given depends on the possible shortage of endogenous glutamine production, it is necessary to determine endogenous production rates both in health and in disease. In contrast to plasma or tissue glutamine concentrations which are static parameters that do not provide any information with regard to production rates, the flux or appearance rate of glutamine is the parameter of interest. Measurement of these prucesses is complicated because many tissues can produce as well as consume glutamine 
at the same time. The hypothesis that glutamine is a conditionaly essential amino acid includes that the production of e.g. muscle falls short for big consumers such as the gut and the liver. Therefore the rate at which glutamine is secreted into the plasma (and taken up under steady state conditions) is the first parameter of interest. This (net) endogenous production rate is also called the whole body production rate or appearance (into the plasma compartment) and employs (stable) isotope technology for its measurement. A second measure is the net flu across organs. This implies the difference between production and disposal of glutamine in the organ of interest, and relies on arteriovenous (AV) balance techniques, in which flux is measured by multiplying $\mathrm{AV}$ concentration differences across an organ of interest times blood flow across that organ. These methodologies will be discussed in more detail in Chapter 10 of this thesis. In the studies presented here, we have made use of (combinations) of these 2 different technologies to assess glutamine metabolism.

By combining results of AV-balance techniques and isotope dilution it is possible to gain substantial more insight in changes taking place in glutamine metabolism under different conditions. Barret and Gelfand introduced a 2 pool model to calculate protein kinetics across an organ 16 . By measurements of substrate concentrations and tracer enrichments in both arterial and venous plasma in combination with plasma tlow across an organ the rate of appearance ( $R a$ ) and disappearance (Rd) of the particular substrate across that organ can be calculated. Figure 3 shows the model they described. Later more complex models were introduced. Biolo et al. described a 3-pool model17 (see figure 3), in which
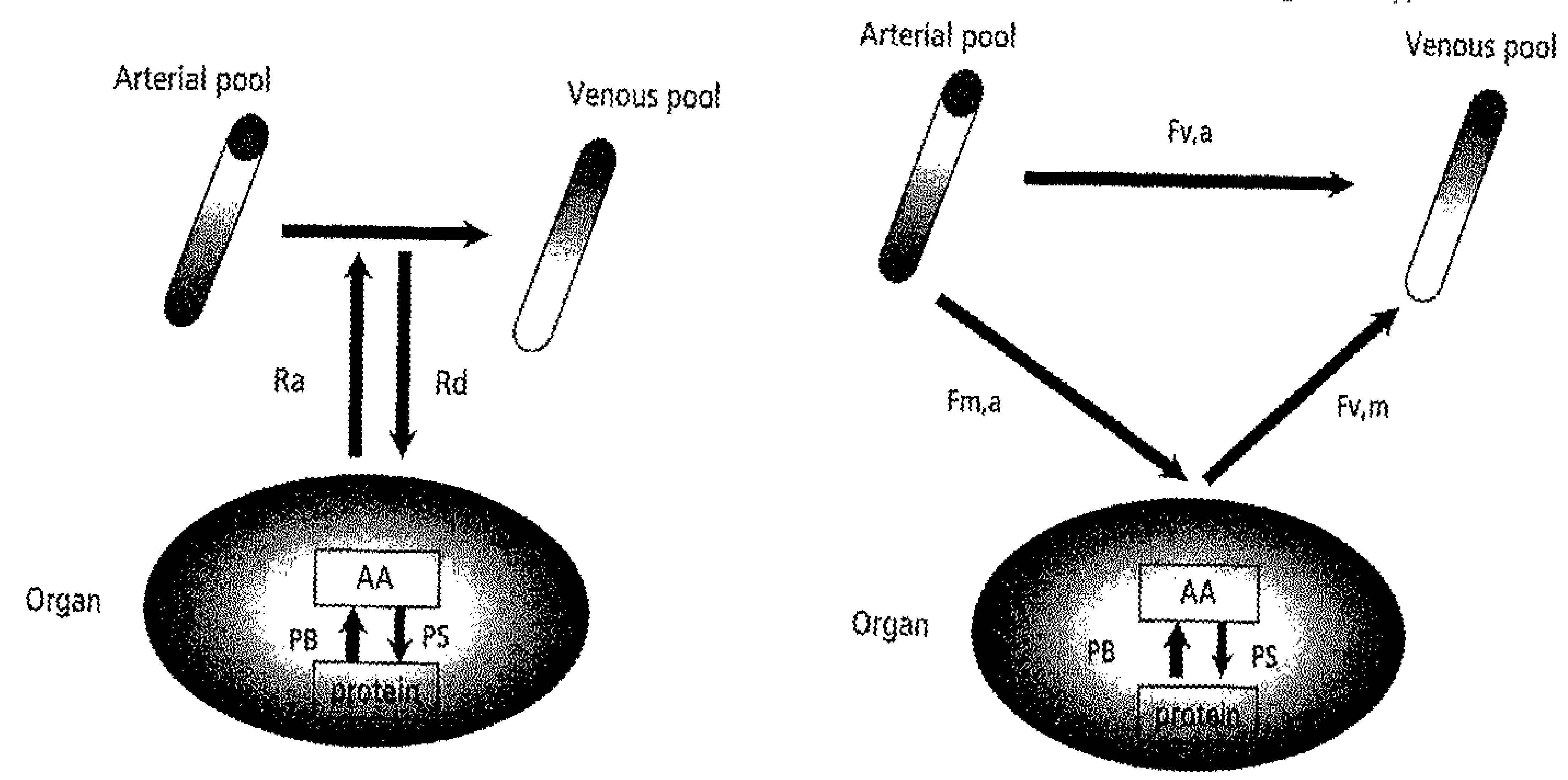

Figure 3 The two-and three pool model for calculation of amino acid kinetics. By measuming arterial (pooli and venous (pooli anrichments, concentrations and flow across an organ, the rate at Which the tracee appears from \{Ra\} or dissappears into (Rol that specific organ can be calculated with the 2 pool model. By adding apprioprate tracers (see text) the contribution of protein synthesis (PS) and protein breakdown (PB) rates can be calculated. AA indicates the intracellular free amino actd pool. Additionaly, in the 3 pool model, the flux of tracee dissapearing from the arterial pool into the intracelutar free amino acid pool / Fm, al, the fux of tracee being released from this atnino acid pool into the venous pool $[\mathrm{K}, \mathrm{m}$ ) and the amount of tracee bypassing the intraceliular compartment (Fv, a) can be calculated. 
meatirements of intracellular enrichments and concentrations of the tracee are incurporated. but even more extensive models have been used. In the 3-pool model calculations can be made to quantify net amino acid movement from artery to the intramusular compartment acid and from muscle compartment to vein, respectively but also th the amount of amino acid that is "shunted" across the organ. In both models, addition of an isotopically labeled essential amino acid (e.g. phenylalanine) provides information on the contribution of protein breakdown and possibly protein synthesis iprovided that the specific amino acid is not oxidized within that organ) to Ra and Rd respectively. However, with an increasing complexity of the models used, the number of assumptions needed to validate the model increase, and the kinetic values become less clear from a physiological point of view. Therefore, in some of the studies presented in this thesis we used only the 2pool model.

Cilutamine metabolism has been studied mostly in animal models, although important differences exist in this regard between humans and especially rodents. We performed the studies described in this thesis in humans when possible, and used a porcine model, which from a metabolic view is better comparable to the human situation than a rodent model, when the invasiveness of some measurements precluded its execution in humans.

The purpose of this thesis was 1. to define the effects of trauma and disease on glutamine metabolism, 2. to address the hypothesis that glutamine is conditionally essential in depleted patients and 3 . to define the consequences of glutamine supplementation on its

In the first part of this thesis we describe 4 studies which were designed to gain more insight into the effects of (surgical) trauma and disease on glutamine metabolism using AVbalance techniques and stable isotope technology. We first describe a study testing the measuring whole body glutamine flux in patients after in the postoperative phase by trauma were studied in more detail in a porcine model trauma on glutamine interorgan metabolism and model. We report the effects of surgical role of the lung in glutamine metabolism in critically il on the gut specifically. Then the describe the individual effects of depletion and inflammation patients is examined. Finally we on glutamine metabolism.

The second part of the thesis concerns the effects of glutamine supplementation. glutamine becomes essential. In depleted patients is one of the conditions during which effects of glutamine supplemented total parenteral nutrition. Then clinical and metabolic supplementation on whole body glutamine flux are reported. Then the effects of glutamine supplementation on interorgan glutamine and gut glutathione mety, the effects of glutamine more detail in a porcine model.

In the discussion, the available knowledge concerning (inter)organ glutamine metabolism is summarized, incorporating the data obtained in the studies described above. 


\section{REFERENCES}

1. Whipple GH. Protein production exchange in the body including hemoglobin, plasma protein and ceil protein. Am I Med Sci. 1938; 196:609

2. Rhoads JE, Dudrick SJ. History of intravenous nutrition. In: Rombeau JL, Caldwell MD, eds. Parenteral nutrition: Saunders, 1993:1-10.

3. Clowes GHA, Randall HT, Cha C-J. Amino acid and energy metabolism in septic and traumatized patients. I Parenter Enteral Nutr 1980; 4:195-205.

4. Clowes GHA, McDermott WV, Williams LF, Loda M, Menzoian JO, Pearl R. Amino acid clearance and prognosis in surgical patients with cirhosis. Surgery 1984; 96:675.085.

5. Studley HO. Percentage weight loss, a basic indicator of surgical risk in patients with chronic peptic ulcer. JAMA 1936; 106:458.460.

6. Giner M, Laviano A, Meguid MM, Gleason JR. In 1995 a correlation between malnutrition and poor outcome in critically ill patients still exists. Nutrition 1996; 12:23-29.

7. Jeejeebhoy KN, Detsky AS, Baker JP. Assessment of nutritional status. JPEN J Parenter Enteral Nutr: $1990 ; 14: 1935-196 \mathrm{~S}$.

8. Schols AM, Mostert R, Soeters PB, Wouters EF, Body composition and exercise performance in patients with chronic obstructive pulmonary disease. Thorax 1991; 46:695.9.

9. Abel RM, Fischer JE, Buckley MJ. Malnutrition in cardiac patients: results of a prospective randomised evaluation of early postoperative total parenteral nutrition. Acta chir Scand 1976; 460:77.

10. van der Hulst RR, von Meyenfeldt MF, van Kreel BK, Thunnissen FB, Brummer RJ, Arends JW, Soeters PB. Gut permeability, intestinal morphology, and nutritional depletion. Nutrition 1998; 14:1-0.

11. Rikimaru T, Taniguchi K, Yartey JE, Kennedy DO, Nkrumah FK. Humoral and cell-mediated immunity in malnourished children in Chana. Eur J Clin Nutr 1998; 52:344-50.

12. Soeters PB. Glutamine: the link between depletion and diminished gut function? I Am Coll Nutr $1990 ; 15: 195 \cdot 190$.

13. Roth E, Zoch G, Schulz F, Karner J, Muhbacher F, Hamilton G, Mauritz W, Sporn P, Funovics J. Amino acid concentrations in plasma and skeletal muscle of patients with acute hemorrhagic necrotizing pancreatitis. Clin Chem 1985; 31:1305-9.

14. Haussinger D, Roth E, lang F; Gerok W. Cellular hydration state: an important determinant of protein catabolism in health and disease. The lancet 1903; 341:1330-1332.

15. Lacey JM, Wilmore DW. Is glutamine a conditionally essential amino acid? Nutr Rev. 1090; 48:297309.

16. Barrett EJ, Revkin JH, Young LH, Zaret BL, Jacob R, Gelfand RA. An isotopic method for measurement of muscle protein synthesis and degradation in vivo. Biochem J 1987; 245:223-8.

17. Biolo $G$, Fleming RYD, Maggi SP, Wolfe RR. Transmembrane transport and intracelullar kinetics of amino acids in human skeletal muscle. Am J Physiol. 1995; 268:E75-E84. 


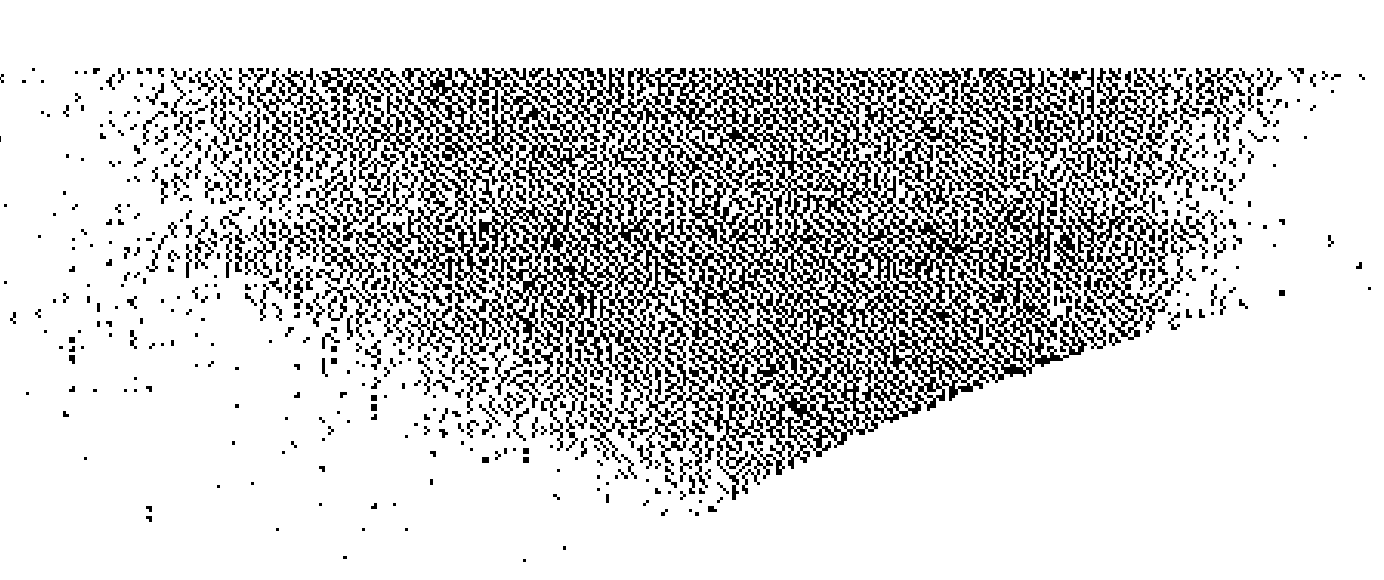

\section{CHAPTER 2}
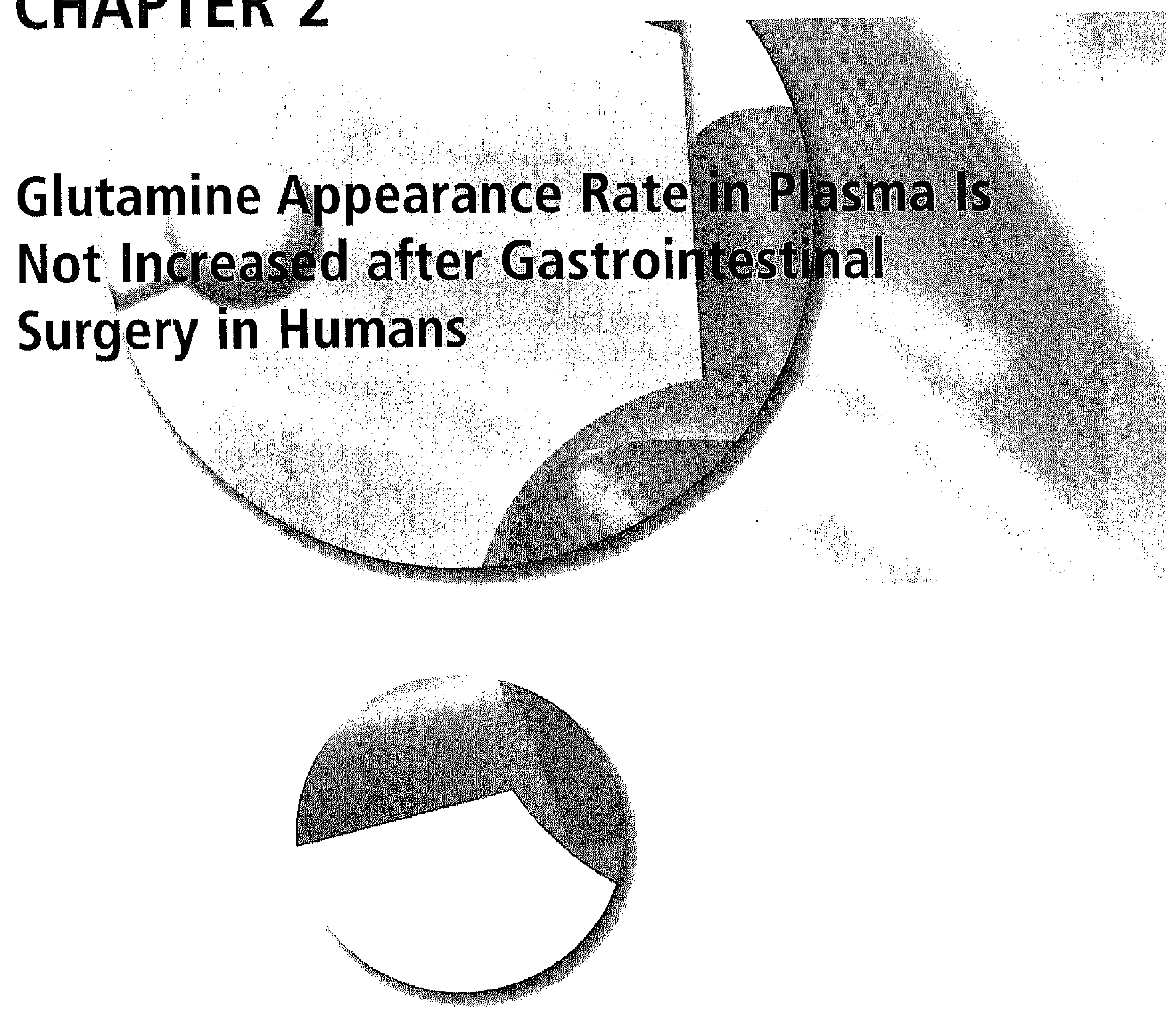

Bernadette A. C. van Acker, Karel W. E. Hulsewé , Anton J. M. Wagenmakers, ${ }^{*}$ Peter B. Soeters and Maarten F. von Meyenfeldt

Department of Surgery, University Hospital Maastricht, Maastricht, The Netherlands; *and Department of Human Biology, Maastricht University, Maastricht, The Netherlands Published in J Nutr 130: 1566-1571, 2000 


\section{ABSTRACT}

The metabolic response to surgical stress is characterized by muscle protein breakdown and mobilization of amino acids and has been postulated to furnish glutamine and other amino acids to the immune system, gut and liver. The present study was undertaken to investigate whether the whole body appearance rate $\left(R_{\mathrm{a}}\right)$ of glutamine in plasma is increased after major elective surgery. Fourteen patients ( 8 males, 6 females) were measured prior to laparotomy and on the second postoperative day. Patients received a primed continuous 6 -hour infusion of $\mathrm{L} \cdot[5.15 \mathrm{~N}] \mathrm{glutamine}$ and $\mathrm{L}-[1.13 \mathrm{C}]$ leucine, and arterial blood samples and muscle biopsies were taken for concentration and enrichment measurements. As expected, the metabolic response to surgery was characterized by a rise in whole body protein breakdown ( $n=14$, $P<0.001)$ and a decreased concentration of glutamine in plasma $(n=14, P<0.001)$ and muscle $(n=8, P<0.01)$. However, these catabolic changes were not reflected by an increase in the plasma Ra of glutamine: $246 \pm 8 \mu \mathrm{mol} / \mathrm{kg} / \mathrm{h}$ before surgery vs. $241 \pm 10 \mu \mathrm{mol} / \mathrm{h}$ on the second postoperative day. We conclude that the whole body $R_{\mathrm{a}}$ of glutamine in plasma is not increased 2 days after elective gastrointestinal surgery. Further studies are warranted to establish whether the lack of an increase in plasma glutamine $R_{\mathrm{a}}$ provides a rationale for glutamine supplementation. 


\section{INTRODUCTION}

The metabolic response to surgical trauma is characterized by increased peripheral protein breakdown. Amino acids are mobilized and taken up by visceral organs for gluconeogenesis and synthesis of acute phase proteins ${ }^{1-4}$. It has been postulated that these processes serve the purpose of supplying fuel for energy generation and building blocks for the synthesis of specific proteins involved in the immunological response and in the restoration of damaged tissue.

Glutamine has a pivotal role as major gluconeogenic precursor and vehicle for interorgan carbon and nitrogen transport 5 . Among the many other functions of glutamine are its role as metabolic substrate for cells with a high turnover rate such as immune cells, enterocytes and renal tubular cells 0,7 , its function as a precursor for purines, pyrimidines and glutathione 8,9 , and its central role in the regulation of acid-base homeostasis 10 . In pigs, we observed an increased muscle glutamine production and a simultaneous rise in glutamine consumption by liver and spleen 1-2 days after surgery 2 . Likewise, in human studies arteriovenous difference measurements across arm or leg have shown a two-fold increase in the net release of glutamine during sepsis or trauma 11.14 , indicating a significant rise in muscle glutamine production. Data in humans regarding the effect of sepsis or trauma on glutamine production from organs and tissues other than muscle are lacking, due to the invasive character of such measurements. We hypothesized that during the metabolic stress of major elective surgery the appearance rate $\left(R_{\mathrm{a}}\right)$ of glutamine at the whole body level is enhanced to meet the increased requirements of the organism for glutamine.

Patients undergoing elective abdominal surgery were measured before operation and on the second postoperative day. Glutamine $R_{\mathrm{a}}$ was determined with the traditional tracer dilution method, using a primed continuous infusion of stable isotope labeled glutamine. Tracer methodology was also used to assess whole body protein turnover in order to relate changes in glutamine $R_{\mathrm{a}}$ to surgery-induced alterations in whole body protein breakdown.

\section{Patients and Methods}

Patients. Fourteen patients (aged 58-78 y, 8 men and 6 women) admitted to the hospital for elective abdominal surgery participated in the study. Underlying diseases were colorectal cancer $(n=6)$, gastric cancer $(n=1)$, pancreatic cancer $(n=1)$, villous adenoma $(n=2)$, diverticular disease $(n=3)$ and Crohn's disease $(n=1)$. No clinical signs of inflammatory activity were present. Body weight averaged $73 \pm 4 \mathrm{~kg}$ and was stable in all subjects, except in three patients who had suffered a recent weight loss of $; 10 \%$ over the last 6 months. Body mass index was $25.9 \pm 1.1 \mathrm{~kg} / \mathrm{m}^{2}$. Written informed consent was obtained from all patients, and the protocol was approved by the Medical Ethics Committee of the University Hospital Maastricht.

Study design. The measurements were performed 1 or 2 days before surgery and on the second postoperative day. Preoperatively, the patients were fasted overnight and remained fasted until the study was completed. In the period between surgery and the postoperative measurement, fluid balance was maintained with normal saline and glucose 
$50 \mathrm{~g} / \mathrm{L}(2-3 \mathrm{~L} /$ day, $850-1250 \mathrm{~kJ}$ per 24 hours $)$ according to standard protocol. Patients had a zero nitrogen intake during this period.

The patients were operated in epidural analgesia with local anesthetics and opiates (bupivacaine and sufentanyl) in addition to general anesthesia. The epidural catheter was left in place during 1-4 days after surgery. In 9 patients the epidural infusion of local anesthetics and opiates was continued during the postoperative measurements. In the other subjects the measurements were carried out after the epidural block had been withdrawn.

In the morning of each study, patients received a catheter in an antecubital vein for isotope infusion and another in the radial artery for blood sampling. The arterial catheter was kept patent by a slow saline infusion. Glucose infusion was stopped during the experiments. At $0830 \mathrm{~h}$ a primed constant intravenous infusion of $L / 5-15 \mathrm{~N} /$ glutamine $10.68 \mu \mathrm{mol} / \mathrm{kg} / \mathrm{h}$; prime $0.68 \mu \mathrm{mol} / \mathrm{kg})$ and $\mathrm{L}\left[1.13 \mathrm{C}\right.$ ]leucine $\left(7.63 \mu \mathrm{mol} / \mathrm{kg}^{-1} / \mathrm{h}^{-1}\right.$ prime $\left.7.63 \mu \mathrm{mol} / \mathrm{kg}\right)$ was given for $o$ hours. The tracers were purchased from Cambridge Isotope Laboratories (Woburn, MA). Blood samples were drawn in chilled-on-ice heparinized tubes before the start of the tracer infusion for measurement of baseline enrichment and at 2, 3, 4, 5 and 6 hours after onset of the infusion. Plasma was obtained by centrifugation of whole blood at $2.200 \mathrm{x}$ g at $4^{\circ} \mathrm{C}$ for $5 \mathrm{~min}$. For the determination of plasma glutamine concentration, plasma was deproteinized with sulfosalicylic acid ${ }^{15}$, mixed with a vortex mixer, frozen in liquid nitrogen and stored at $-80^{\circ} \mathrm{C}$. For tracer enrichment measurements, plasma was frozen and stored at $-80^{\circ} \mathrm{C}$ until analysis. In 8 patients, percutaneous muscle biopsies were taken from the anterior tibial muscle once or twice during each tracer infusion to measure glutamine enrichment and concentration in the intracellular free glutamine pool in muscle. Biopsies were taken using the conchotome technique ${ }^{16}$. Blood, visible fat and connective tissue were quickly removed from the specimen, and the tissue was immediately frozen in liquid nitrogen and subsequently stored at $-80^{\circ} \mathrm{C}$ for later analysis. To limit the number of biopsies in each patient, baseline enrichment in muscle was assumed to be equal to the enrichment in plasma. Support for this assumption comes from a previous study in a similar group of preoperative patients. By extrapolating back to time zero the glutamine enrichment data in successive muscle biopsies taken in the course of 11 -hours glutamine tracer infusion studies, it was shown that glutamine baseline enrichment in muscle equaled that of plasma 17.

Analytical methods. The biopsies were freeze-dried and further freed from adherent blood and connective tissues. The water content of the biopsies was calculated from the weight difference before and after freeze-drying and used for conversion from dry to wet weight. The muscle specimens were powdered and deproteinized using a Mini-Beadbeater (Biospeck Products, Bartlesville, OK). Of the pulverized tissue, $\sim 30 \mathrm{mg}$ was added to 400 $\mu \mathrm{L}$ sulfosalicylic acid $50 \mathrm{~g} / \mathrm{L}$ and $0.1 \mathrm{~g}$ glass beads (diameter $1 \mathrm{~mm}$; Biospeck Products) and beaten for $50 \mathrm{~s}$. The supernatant was frozen in liquid nitrogen and stored at $-80^{\circ} \mathrm{C}$ until later analysis. The concentration of glutamine in supernatant, plasma and tracer infusate was determined by fully automated HPLC ${ }^{18}$. Muscle intracellular glutamine concentration was expressed per liter intracellular water 10,20 .

The is enrichment of plasma and muscle glutamine was determined using a tertbutyldimethy]silyl derivative and a gas chromatographic combustion isotope ratio mass spectrometry (Finnigan MAT 252, Bremen, Germany), as previously described ${ }^{17}$. Plasma enrichments of a ketoisocaproate $(\mathrm{KIC})$ were measured using a quinoxalinoltrimethylsilyl 
derivative and a gas chromatographic-mass spectrometric system (Finnigan Incos XL, San Jose, CA), in a similar manner to that described previously ${ }^{21}$. Final values for KIC determinations were corrected using calibration curves.

Calculations. The $R_{\mathrm{a}}$ of glutamine into plasma $\left(R_{\mathrm{a}, \mathrm{gn}}\right.$, in $\left.\mu \mathrm{mol} / \mathrm{kg} / \mathrm{h}\right)$ was calculated as:

$$
R_{\mathrm{a}, \mathrm{gln}}=\mathrm{i}_{\mathrm{l}} 15_{\mathrm{N} \mid \mathrm{gln}} \mathrm{X}\left[\left(\mathrm{E}_{\mathrm{i}, \mathrm{gln}} / \mathrm{E}_{\mathrm{p}, \mathrm{gln}}\right)-1\right]
$$

where $\mathrm{i}_{1}{ }^{15} \mathrm{Ngghn}$ is the tracer infusion rate in $\mu \mathrm{mol} / \mathrm{kg} / \mathrm{h}, \mathrm{E}_{\mathrm{i}, \mathrm{gn}}$ is the glutamine enrichment in the tracer infusate, expressed in mole percentage excess (MPE), and $E_{p, g n}$ is the mean plasma glutamine enrichment between 2 and 6 hours of tracer infusion. As demonstrated before, the rate of glutamine appearance obtained in this way overestimates the true appearance rate of glutamine in plasma by at least $20 \%$ because of slow equilibration of the glutamine tracer with the large muscle glutamine pool (van Acker et al. 1998). Proteolysis was measured using the whole body $R_{\mathrm{a}}$ of leucine $\left(R_{\mathrm{a} \text { leu }}\right.$ in $\left.\mu \mathrm{mol} / \mathrm{kg} / \mathrm{h}\right) . R_{\mathrm{a}}$ leu was calculated using plasma KIC enrichments 22:

$$
R_{\mathrm{a} \text { leu }}=\mathrm{i}_{\mathrm{l}} 13_{\text {Clleu }} \mathrm{x}\left[\left(\mathrm{E}_{\mathrm{i}, \text { leu }} / \mathrm{E}_{\mathrm{p}, \mathrm{kic}}\right)-1\right]
$$

where $\mathrm{i}_{1} 1_{\mathrm{Clleu}}$ is the tracer infusion rate in $\mu \mathrm{mol} / \mathrm{kg} / \mathrm{h}, \mathrm{E}_{\mathrm{i}, \text { leu }}$ is the leucine enrichment (MPE) in the tracer infusate and $E_{p, k i c}$ is the average plasma KIC enrichment from 2 to 6 hours of tracer infusion. In the absence of exogenous amino acids, the calculated $R_{\mathrm{a}}$ by definition equals the endogenous $R_{\mathrm{a}}$.

Glutamine arising from protein degradation $\left(P D_{\mathrm{gln}}\right)$ was calculated as:

$$
P D_{\text {gln }}=R_{\text {aleu }} \times(4.4 \times 131) /(8 \times 146)
$$

where 4.4 and 8 are the assumed glutamine and leucine contents of body protein ( $g$ per $100 \mathrm{~g}$ of protein), respectively, and 146 and 131 are glutamine and leucine molecular weights $(\mathrm{g} / \mathrm{mol})$, respectively 23 .

The intracellular concentration of glutamine [GIn]i was calculated by subtracting the free extracellular part from the total amount $\left(G n_{m}\right)$, as previously described ${ }^{19}$ :

$$
[\mathrm{Gln}]_{\mathrm{i}}=\left(1.000 \times \mathrm{Gln}_{\mathrm{m}}-\mathrm{H}_{2} \mathrm{O}_{\mathrm{e}} \times[\mathrm{Gln}]_{\mathrm{p}}\right) / \mathrm{H}_{2} \mathrm{O}_{\mathrm{i}}
$$

assuming the plasma concentration $[G \ln ]_{\mathrm{p}}$ to be equal to the concentration in the interstitial fluid. Regarding the preoperative measurements, the extracellular $\left(\mathrm{H}_{2} \mathrm{O}_{\mathrm{e}}\right)$ and intracellular $\left(\mathrm{H}_{2} \mathrm{O}_{\mathrm{i}}\right)$ water content (mL/kg muscle) were assumed to amount to 13 and $87 \%$ of the total water content of muscle tissue ${ }^{19}$. Concerning the increased extracellular and decreased intracellular water content observed after surgical trauma 20 , values of $18 \%$ $\left(\mathrm{H}_{2} \mathrm{O}_{\mathrm{e}}\right)$ and $82 \%\left(\mathrm{H}_{2} \mathrm{O}_{\mathrm{i}}\right)$ were used for the measurements performed on the second postoperative day. Values of intracellular enrichment were calculated in a similar manner.

Statistics. Data are given as means \pm SEM, unless stated otherwise. Comparisons between pre- and postoperative values were made using the Wilcoxon matched pairs signed rank sum test. The Mann-Whitney $U$ test was used for comparing data from different 
patient groups (i.e., patients with and without cancer, patients with and without epidural anesthesia). Regarding the time course of glutamine and $\mathrm{KIC}$ enrichment in plasma, a repeated measures ANOVA was performed to detect effects of time and surgical treatment. A $P$-value of $<0.05$ was considered significant.

\section{RESULTS}

Plasma [1.5 N/glutamine and $[1.13 \mathrm{C}] \mathrm{KIC}$ enrichments before and after surgery are shown in Figure 1. A plateau in enrichment was achieved for KIC but not for glutamine. Plasma glutamine enrichment increased slowly but significantly with tracer infusion time $(P<0.05)$, indicating that isotopic steady state had not been reached. Similar values were obtained for preand postoperative glutamine enrichment in plasma. On the other hand, plasma KIC enrichment decreased after surgery $(P<0.001)$, reflecting increased dilution of the leucine tracer by endogenous amino acids. When calculating whole body protein breakdown rates, individual patients showed higher values on the second postoperative day than during the preoperative study. On average, leucine $R_{a}$ increased by $26 \pm 5 \%$, from $91 \pm 3 \mu \mathrm{mol} / \mathrm{kg} / \mathrm{h}$ before operation to $115 \pm 6 \mu \mathrm{mol} / \mathrm{kg} / \mathrm{h} 2$ days after surgery (Fig. $2, P<0.001$ ). Despite the rise in whole body protein breakdown, no increase was observed in the $R_{a}$ of glutamine in plasma (Fig. 3). Whole body glutamine $R_{a}$ in plasma averaged $240 \pm 8$ and $241 \pm 10 \mu \mathrm{mol} / \mathrm{kg} / \mathrm{h}$ during the pre- and postoperative measurement, respectively, with some patients showing no change, some showing an increase and some a decrease in glutamine $R_{\mathrm{a}}$. The calculated amount of glutamine $R_{a}$ arising from proteolysis increased in each patient, and averaged $45 \pm 1 \mu \mathrm{mol} / \mathrm{kg}^{-1} / \mathrm{h}^{-1}$ before and $57 \pm 3 \mu \mathrm{mol} / \mathrm{kg} / \mathrm{h}$ on day 2 after surgery $(P<0.001)$.

The rates of protein breakdown and glutamine $R_{\mathrm{a}}$ were not different between patients with cancer and those without, either before or after the surgical trauma (Table 1). Also, similar values were obtained in patients with recent weight loss and patients with a stable

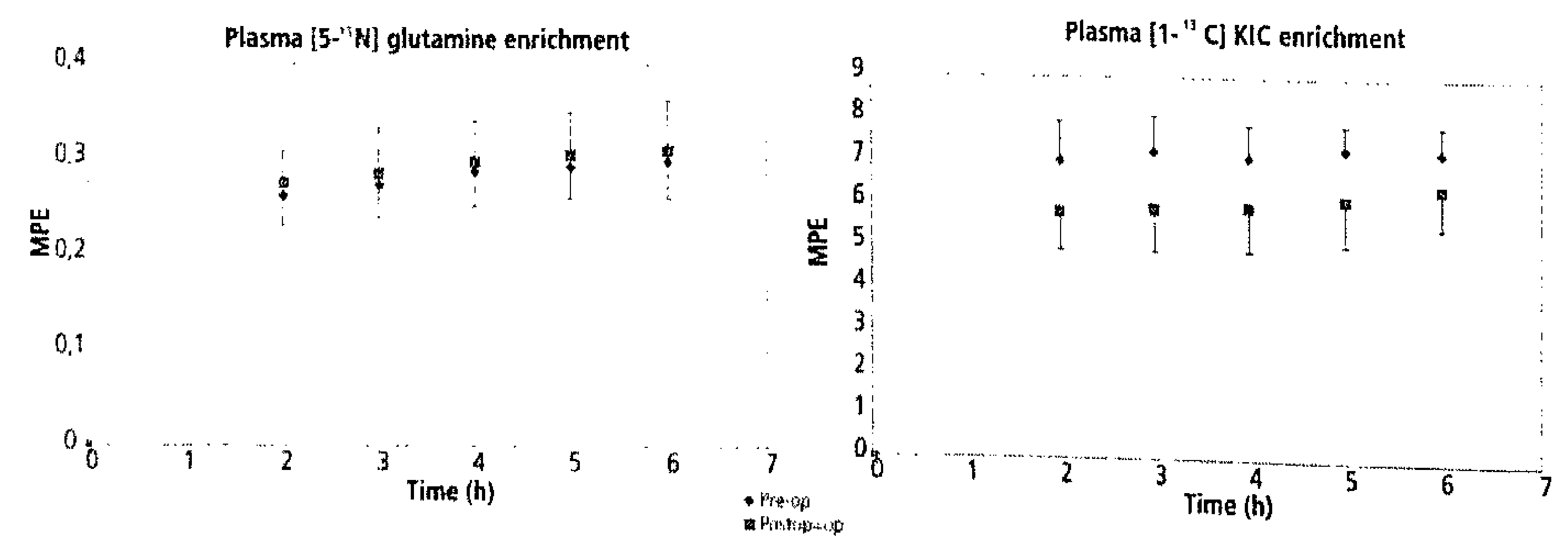

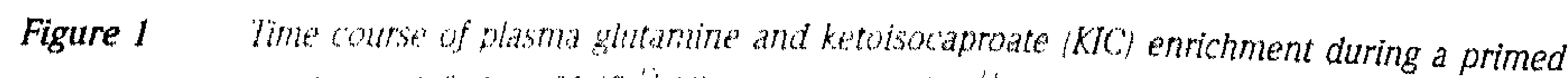

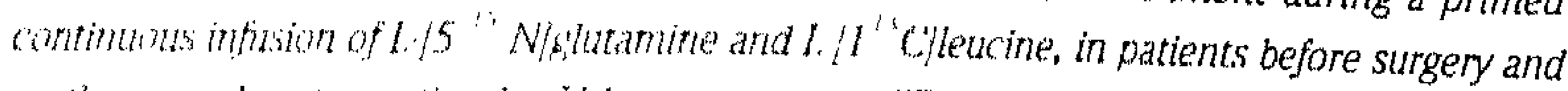

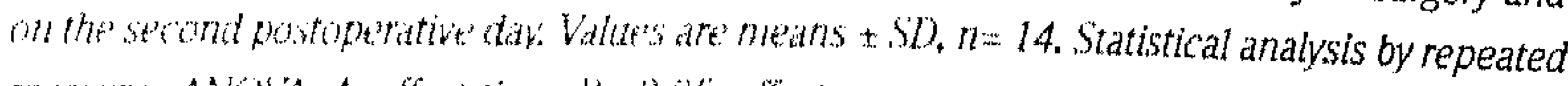

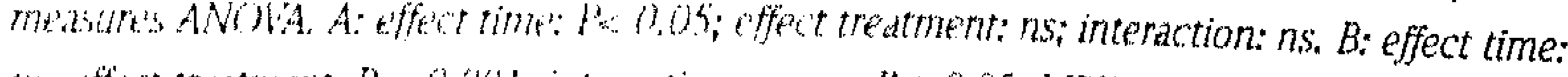

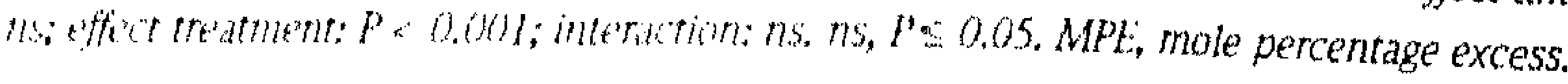


weight. The presence of epidural anesthesia did not affect the postoperative changes in leucine and glutamine $R_{\mathrm{a}}$ : leucine $R_{\mathrm{a}}$ increased by $30 \pm 6 \%$ and $20 \pm 6 \%$ in the patients with and without epidural anesthesia, respectively, whereas glutamine $R_{\mathrm{a}}$ did not change in either group $(-4 \pm 6 \%$ and $-1 \pm 3 \%)$.

Leucine Ra

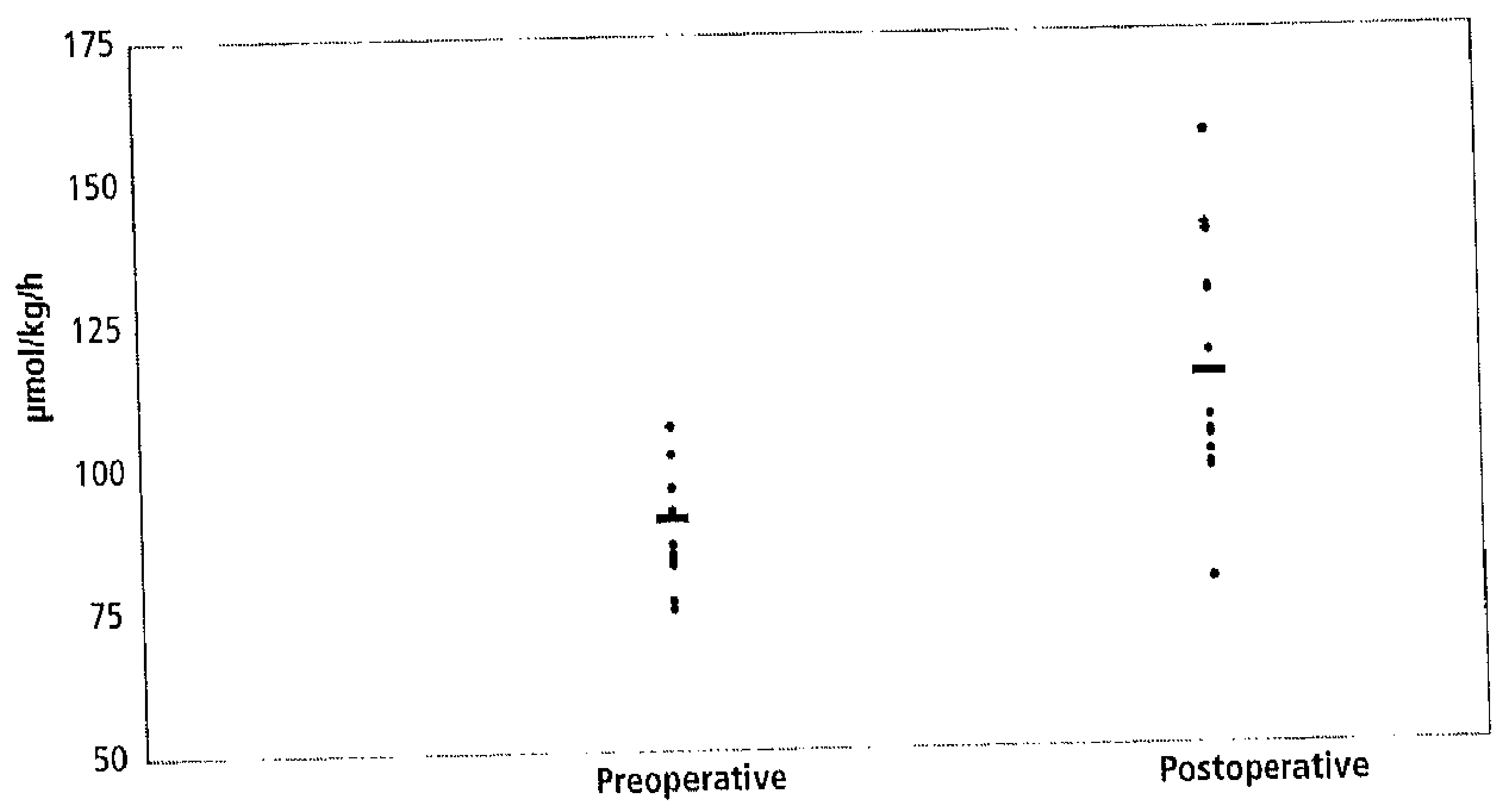

Figure 2 Whole body protein breakdown rates of patients, measured by the appearance rate $\left(R_{a}\right)$ of leucine before and 2 days after surgery. Individual data are given before surgery and on the second postoperative day. Mean values are represented by a horizontal line, $n=14$. Wilcoxon signed ranks test, preoperative vs. postoperative: $P<0.001$.

Glutamine Ra

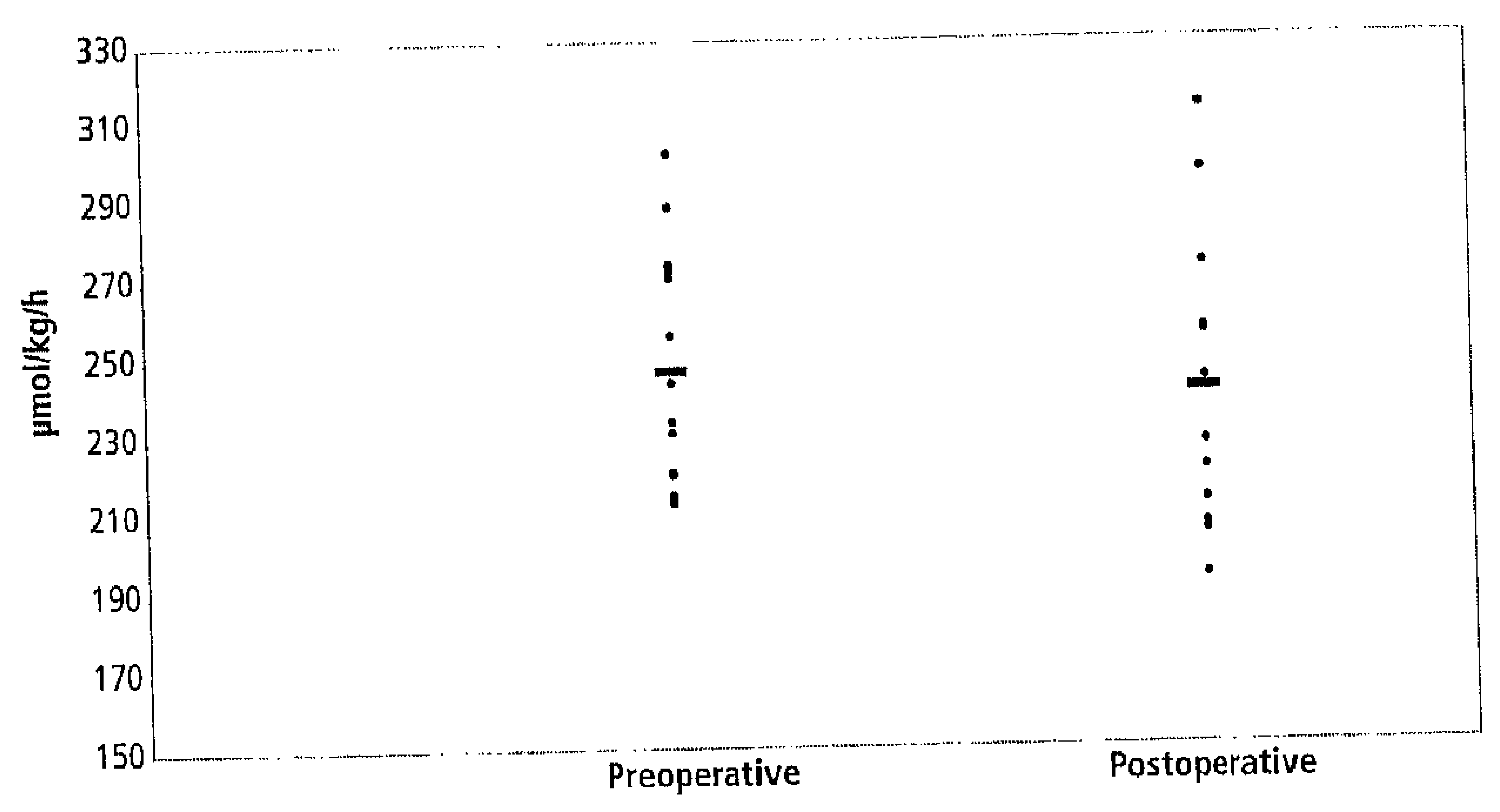

Figure 3 Whole body glutamine appearance rate $\left(R_{a}\right)$ of patients before and 2 days after surgery. Individual data are given before surgery and on the second postoperative day. Mean values are represented by a horizontal line, $n=14$. Wilcoxon signed ranks test, preoperative vs. postoperative: $P \leq 0.05$. 
Table 1 Whole body glutamine appearance rate and whole body protein breakdown before and 2 days after surgery in patients with and without gastrointestinal cancer'

Preoperative

Postoperative

\begin{tabular}{lcccc}
\hline & Cancer & Noncancer & Cancer & Noncancer \\
\hline Glutamine $R_{a}$ & \multicolumn{5}{c}{$\mu$ mol/kg/h } \\
Leucine $R_{a}$ & $248 \pm 12$ & $244 \pm 14$ & $240 \pm 17$ & $242 \pm 11$ \\
\hline
\end{tabular}

1 Means \pm SEM, $n=8$ (cancer) or 6 (noncancer). $R_{a}=$ appearance rate. Statistical analysis by Mann-Whitney $U$ test and Wilcoxon signed ranks test. Preoperative cancer vs. noncancer; Glutamine $R_{a}$, ns; Leucine $R_{a}$, ns; Postoperative vs. preoperative, cancer: Glutamine $R_{a}$ ns; Leucine $R_{a}, P<0.05$. Postoperative vs, preoperative, noncancer: Glutamine $R_{a}$, ns; Leucine $R_{a}, P<0,05$, ns, $P \leq 0.05$.

After surgery, the concentration of glutamine in plasma decreased in every patient. On the second postoperative day, the average decrease in plasma glutamine concentration was $31 \pm 3 \%$, from $625 \pm 22 \mu \mathrm{mol} / \mathrm{L}$ to $431 \pm 17 \mu \mathrm{mol} / \mathrm{L}$ ( Fig. $4, P<0.001$ ). Hematocrit decreased by $12 \pm 2 \%$, from $0.40 \pm 0.01$ to $0.35 \pm 0.01(P<0.001)$, indicating that part of the reduction in plasma glutamine may be attributed to postsurgical hemodilution. $\mathrm{A}$ decrease also occurred in the concentration of intramuscular free glutamine: from $10.82 \pm$ 0.75 to $7.69 \pm 0.59 \mathrm{mmol} / \mathrm{L}$ intracellular water, a decline of $27 \pm 6 \%$ (Fig. $5, P<0.01$ ). No change was observed in the total water content in muscle tissue: $759 \pm 19 \mathrm{~mL} / \mathrm{kg}$ muscle before surgery vs. $756 \pm 16 \mathrm{~mL} / \mathrm{kg}$ muscle 2 days after surgery.

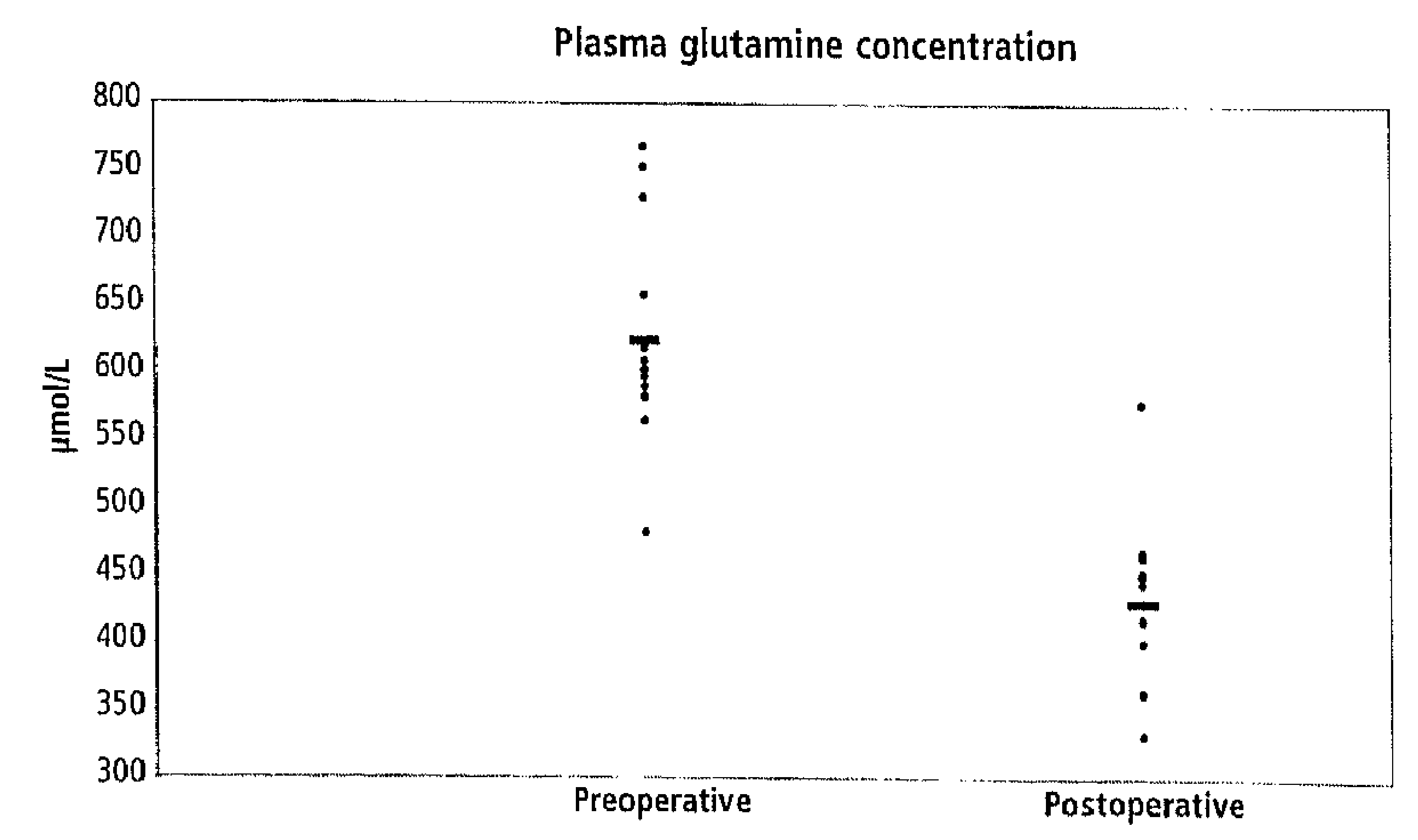

Figure 4 Glutamine concentration in plasma of patients before and 2 days after surgery. Individual data are given. Mean values are represented by a horizontal line, $n=14$. Wilcoxon signed ranks test, preoperative vs. postoperative: $P<0.001$. 


\section{Intracellular glutamine concentration}

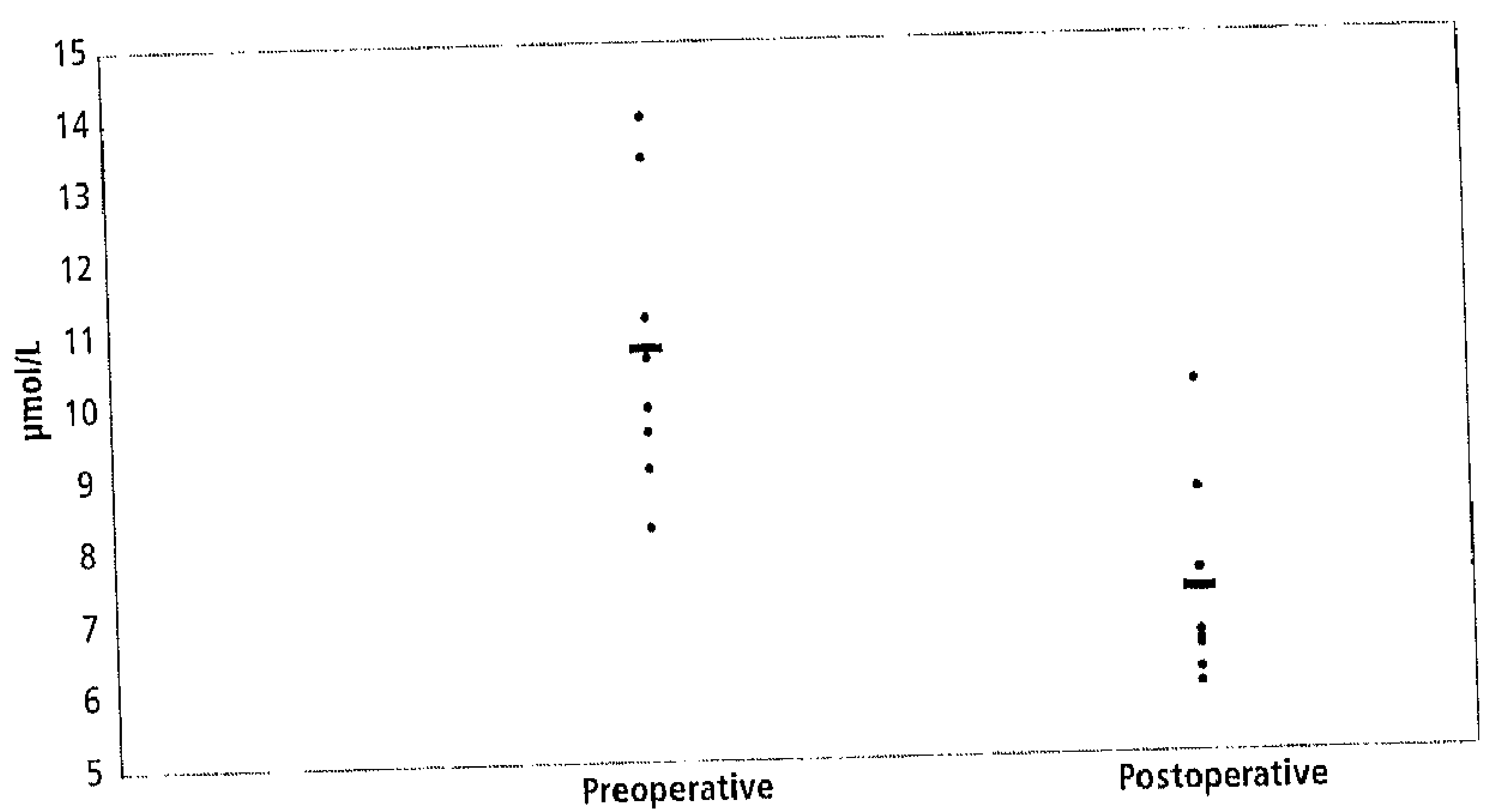

Figure 5

Glutamine concentration in the intramuscular free glutamine pool of patients before and 2 days after surgery. Individual data are given and expressed in mmol/L intracellular water. Mean values are represented by a horizontal line, $n=8$. Wilcoxon signed ranks test, preoperative vs. postoperative: $P<0.01$.

As a result of the reduced free glutamine pool in muscle, on the second postoperative day higher levels of intramuscular glutamine enrichment were observed toward the end of each tracer infusion: $0.055 \pm 0.006$ vs. $0.038 \pm 0.005 \mathrm{MPE}$ preoperatively (Fig. 6, $P<0.05$ ). When the postoperative reduction in the size of the intramuscular free glutamine pool was taken into account, similar amounts of labeled glutamine were found in muscle after 6 hours of tracer infusion: $4.0 \pm 0.6$ and $4.1 \pm 0.5 \mu \mathrm{mol} / \mathrm{L}$ intracellular water during pre- and postoperative measurements, respectively. As shown in Figure 6, in four postoperative patients an additional biopsy was taken $\sim 3$ hours after start of tracer infusion. The rate of rise of labeled glutamine in muscle calculated from the consecutive biopsies was $0.56 \pm 0.05 \mu \mathrm{mol} / \mathrm{L}$

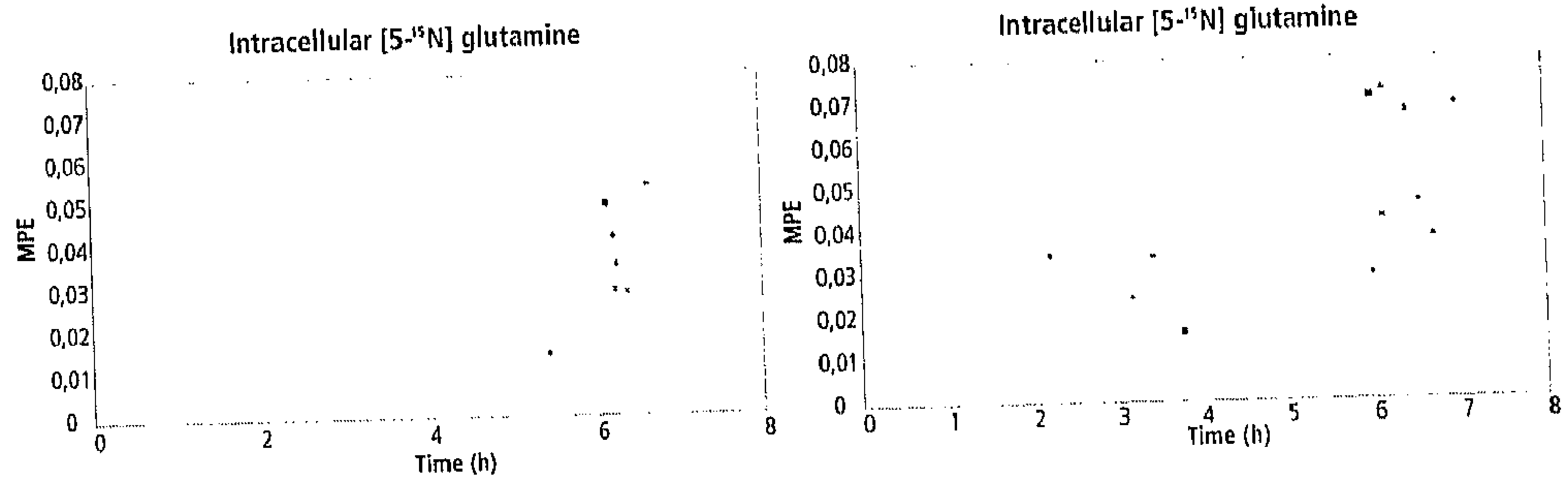

Figure 6 Intramuscular glutamine enrichment in patients during a primed continuous infusion of $L-15$ ${ }^{15}$ Nglutamine. Individual data are given and expressed in mole percentage excess (MPE), $n=8$. Wilcoxon signed ranks test, $(A)$ preoperative $v s$. (B) postoperative enrichment at $t-6$ hours: $P<0.01$. 
intracellular water $h$ between 3 and 6 hours of tracer infusion. When, in these patients, the rate of rise of labeled glutamine in muscle was calculated on the basis of a single biopsy at 6 hours assuming equal entichment in plasma and muscle at baseline, a similar value was obtained $(0.58 \pm 0.09 \mu \mathrm{mol} / \mathrm{L}$ intracellular water $\mathrm{h})$, suggesting that the assumption was valid.

\section{Discussion}

The changes in protein breakdown and in plasma and intramuscular glutamine concentration observed in this study are consistent with the literature concerning the metabolic effects of elective abdominal surgery 11,20 . The catabolic stress response was not arrested by continuous epidural infusion of local anesthetics, presumably as a result of the $60-70 \%$ lower dose of bupivacaine used in the present study as compared to previous investigations on the effect of epidural anesthesia 24 . Proteolysis of skeletal muscle is a major contributor to the increase in whole body protein breakdown observed 2 days after surgery ${ }^{1}$. The decrease in the concentration of glutamine in skeletal muscle is a highly consistent finding after trauma 20.25. Quantitatively, this is a considerable reduction, because muscle glutamine represents more than half of the total free amino acid pool in the human body. Essén et al. observed a $21 \%$ decline in the concentration of glutamine in muscle during the first 24 hours after surgery 25 . This would indicate that the losses of glutamine from the intracellular free glutamine pool occur mainly during the first postoperative day, and to a smaller extent during the following days.

The rise in protein breakdown and the shrinking plasma and intracellular glutamine pools 48 hours after laparotomy are consistent with the concept of an increased metabolic demand of glutamine in the postoperative period. Nevertheless, this remains coniectural because it is not fully known how intracellular glutamine levels are maintained and how this process is regulated. Other possibilities may include an impairment of membrane integrity, a loss of the $\mathrm{Na}^{+}$electrochemical gradient and/or changes in the activity of the glutamine transporter by trauma 26,27 . Evidence exists that tissues involved in the immune system, such as liver and spleen, take up increased amounts of glutamine in response to trauma ${ }^{2}$. Previous studies in humans have shown an increased net release of glutamine from muscle during periods of elevated metabolic stress, such as during sepsis ${ }^{12}, 3$ to 5 days after major trauma ${ }^{12}$, immediately after elective cholecystectomy ${ }^{14}, 4$ days after hysterectomy ${ }^{11}$ and on the day 2 after elective gastrointestinal surgery in a group of patients similar as the present study group 13. On the other hand, utilizing tracer kinetic techniques, we did not observe a rise in the $R_{\mathrm{a}}$ of glutamine in plasma 2 days after surgery. Our findings are in agreement with a recent study in critically ill patients, showing an unaltered plasma glutamine $R_{\mathrm{a}}$ when compared to matched healthy controls, despite a major increase in proteolysis and decrease in plasma glutamine concentration 28 . A discrepancy between the $R_{\mathrm{a}}$ of glutamine in plasma and the net release of glutamine from muscle has previously been observed in our laboratory: in rats, the surgery-induced rise in the net release of muscle glutamine was not matched by an increased plasma $R_{\mathrm{a}}{ }^{29}$. 
In the absence of exogenous glutamine, the sources that contribute to the $R_{\mathrm{a}}$ of glutamine are proteolysis, de novo synthesis and glutamine losses from the free intracellular pool. Because glutamine derived from proteolysis was increased after surgery, a decline in the rate of glutamine de novosynthesis and/or a reduction in the loss from the free intracellular pool are factors that might explain the absence of any change in the whole body glutamine $R_{\mathrm{a}}$. Hankard et al. recently observed an $\sim 11 \%$ decline in whole-body glutamine $R_{\mathrm{a}}$ between 18 and 42 hours of fasting, entirely accounted for by a drop in the estimated rates of glutamine de novo synthesis ${ }^{30}$. In the present study, the preoperative measurements were conducted after an overnight fast, whereas during the postsurgical measurements patients were in a near-fasted state for about 80 hours. Only a limited amount of glucose was provided in the immediate postoperative days, which is the postoperative clinical routine in patients undergoing gastrointestinal surgery. In theory, therefore, this period of fasting may have played a role in our observation that the plasma $R_{\mathrm{a}}$ of glutamine was not increased on the second postoperative day.

Although skeletal muscle is the main glutamine-producing tissue lin humans $>60 \%$ of endogenous glutamine $R_{\mathrm{a}}$ is released by muscle 5,31 , other sources such as liver, brain, lung and adipose tissue also contribute to the $R_{\mathrm{a}}$ of glutamine in plasma measured by tracer dilution techniques. An alternative explanation for the unchanged whole body glutamine $R_{\mathrm{a}}$ after laparotomy, therefore, is that any surgery-induced rise in muscle glutamine release might have been offset by a decreased release from sources other than muscle, such as liver. Increased consumption of glutamine produced within the tissue itself, before exchange has occurred with the systemic circulation, may contribute to the diminished release. In a previous study in pigs, we showed that the liver switches from net production of glutamine in the preoperative setting to net consumption of glutamine after surgery 2. Part of the glutamine consumed by the liver may be used for proliferation of Kupfer cells and synthesis of acute phase proteins, another part for giuconeogenesis.

With respect to the muscle data, we assumed similar glutamine baseline enrichment values in muscle and plasma. Support for this assumption comes from the consecutive muscle biopsies taken here and in a previous study ${ }^{17}$. By extrapolating the successive glutamine enrichment data back to time zero, it was shown that the baseline enrichment in muscle approximated that in plasma, both in pre-and postoperative patients. As a result of the slow equilibration of the glutamine tracer with the large muscle glutamine pool, the $R_{\mathrm{a}}$ of glutamine in plasma overestimates the whole-body glutamine flux ${ }^{17}$. In this study, too, isotopic steady-state conditions were not achieved during the 6-h infusion of glutamine tracers, neither before nor after surgery. However, similar amounts of glutamine tracer were retained in the intramuscular free glutamine pool during the two measurements, i.e., before and 2 days after surgery. This indicates that the degree in which the true glutamine flux is overestimated by the measured glutamine $R_{a}$ probably is of the same magnitude for both pre- and postoperative measurements.

In conclusion, plasma and muscle glutamine concentrations are decreased in patients 48 hours after laparotomy. These findings have been suggested to result from increased uptake by the immune system. An increased plasma glutamine $R_{\mathrm{a}}$ was not shown in our patients, despite the fact that previous studies in animal models and humans have shown an increased net release of glutamine from muscle following trauma or infection. One of 
the explanations for the absence of an increase in plasma glutamine $R_{\mathrm{a}}$ may be a decreased release from nonmuscular tissues (e.g., liver) or a decreased de novo synthesis of glutamine in muscle. Further research is warranted to elucidate whether absence of an increase in the plasma glutamine $R_{a}$ of these patients provides a rationale for glutamine supplementation. A reduction in the de novo synthesis of glutamine may reflect shortage of substrate for glutamine synthesis in muscle (e.g., glutamate and muscle protein derived amino acids). In that case it is likely that patients undergoing gastrointestinal surgery will benefit from glutamine supplementation in the first few days after surgery.

\section{ACKNOWLEDGEMENTS}

The authors acknowledge the technical assistance of M. Meers, A. P. Gijsen, F. van de Vegt, A. Rousseau, H. M. H. van Eijk and D. R. Rooyakkers. 


\section{REFERENCES}

1. Clowes GHA, George BC, Villee CA, Saravis CA. Muscle proteolysis induced by a circulating peptide in patients with sepsis or trauma. New England Journal of Medicine. 1983; 308:545-552.

2. Deutz NEP, Reijven PL, Athanasas G, Soeters PB. Post-operative changes in hepatic, intestinal, splenic and muscle fluxes of amino acids and ammonia in pigs. Clin Sci Colch. 1992; 83:607-614.

3. Douglas RG, Shaw JH. Metabolic response to sepsis and trauma. Br J Surg. 1989; 76:115-122.

4. Wannemacher RW, Jr. Key role of various individual amino acids in host response to infection. Am J Clin Nutr. 1977; 30:1209-1280.

5. Nurjhan N, Bucci A, Periello G, et al. Glutamine; a major gluconeogenic precursor and vehicle for interorgan carbon transport in man. J Clin Invest. 1995; 95:272.277.

6. Ardawi MS, Newsholme EA. Glutamine metabolism in lymphocytes of the rat. Biochem J. 1983; 212:835-842.

7. Windmueller HG. Glutamine utilization by the small intestine. Adv Enzymol. 1982:201-237.

8. Cao Y, Feng Z, Hoos A, Klimberg VS. Glutamine enhances gut glutathione production. JPENJ Parenter Enteral Nutr. 1998; 22:224-227.

9. Lacey JM, Wilmore DW. Is glutamine a conditionally essential amino acid? Nutr Rev. 1990; 48:297309.

10. Halperin ML, Ethier JH, Kamel KS. The excretion of ammonium ions and acid base balance. Clin Biochem. 1990; 23:185-188.

11. Carli F, Webster J, Ramachandra V, et al. Aspects of protein metabolism after elective surgery in patients receiving constant nutritional support. Clin Sci (Lond). 1990; 78:621.628.

12. Clowes GHA, Randall HT, Cha C-J. Amino acid and energy metabolism in septic and traumatized patients. J Parenter Enteral Nutr: 1980; 4:195-205.

13. Mjaaland M, Unneberg K, Larsson J, Nilsson L, Revhaug A. Growth hormone after abdominal surgery attenuated forearm glutamine, alanine, 3-methylhistidine, and total amino acid efflux in patients receiving total parenteral nutrition. Ann Surg. 1993; 217:413-422.

14. Stjernstrom $H$, Lund $J$, Wiklund $L$, et al. The influence of abdominal surgical trauma on the exchange of blood-borne amino acids in the human leg. Clin Nutr: 1986; 5:123-131.

15. Van Eijk HM, Dejong CHC, Deutz NEP, Soeters PB. Influence of storage conditions on normal plasma amino acid concentrations. Clin Nutr. 1994; 13:374-380.

16. Dietrichson P, Coakley J, Smith PE, Griffiths RD, Helliwell TR, Edwards RH. Conchotome and needle percutaneous biopsy of skeletal muscle. J Neurol Neurosurg Psychiatry. 1987; 50:1461-1467.

17. Van Acker BAC, Hulsewé KWE, Wagenmakers AJM, et al. Absence of glutamine isotopic steady state: implications for the assessment of whole-body glutamine production rate. Clin Sci (Colch). 1998; 95:339-346.

18. van Eijk HM, Rooyakkers DR, Deutz NE. Rapid routine determination of amino acids in plasma by high- performance liquid chromatography with a 2-3 microns Spherisorb ODS II column. $J$ Chromatogr. 1993; 620:143-148.

19. Bergstrom J, Furst P, Noree LO, Vinnars E. Intracellular free amino acid concentration in human muscle tissue. J Appl Physiol. 1974; 36:693-697.

20. Vinnars E, Bergstrom J, Furst P. Influence of the postoperative state on the intracellular amino acids in human muscle tissue. Ann Surg. 1975; 182:665-671. 
21. Ford GC, Cheng KN, Halliday D. Analysis of (1-13C)leucine and (13C)KIC in plasma by capillary gas chromatography/mass spectrometry in protein turnover studies. Biomed Mass Spectrom. 1985; $12: 432-436$.

22. Horber FF, HorberFeyder CM, Krayer S, Schwenk WF, Haymond MW. Plasma reciprocal pool specific activity predicts that of intracellular free leucine for protein synthesis. Am J Physiol. 1989; 257:E385.399.

23. Kuhn KS, Schuhmann K. Stehle P, Darmaun D, Furst P. Determination of glutamine in muscle protein facilitates accurate assessment of proteolysis and de novo synthesis-derived endogenous glutamine production. Am J Clin Nutr. 1999; 70:484-489.

24. Carli F, Halliday D. Modulation of protein metabolism in the surgical patient. Effect of 48-hour continuous epidural block with local anesthetics on leucine kinetics. Reg Anesth. 1996; 21:430-435.

25. Essen P. Wernerman J, Sonnenfeld T, Thunell S, Vinnars E. Free amino acids in plasma and muscle during 24 hours post-operatively--a descriptive study. Clin Physiol. 1992; 12:163-177.

25. Hundal HS, Rennie MJ, Watt PW. Characteristics of L-glutamine transport in perfused rat skeletal muscle. J Physiol. 1987: 393:283.305.

27. Rennie MI, Hundal HS, Babil P, et al. Characteristics of a glutamine carrier in skeletal muscle have important consequences for nitrogen loss in injury, infection, and chronic disease. Lancet. 1986; 2:1008-1012.

28. Jackson NC, Carroll PV, Russell.Jones DL, Sonksen PH, Treacher DF, Umpleby AM. The metabolic consequences of critical illness: acute effects on glutamine and protein metabolism. Am J Physiol. 1999; 276:E:163.170.

29. Blaauw de I, Deutz NEP, von Meyenfeldt MF. Cancer reduces the metabolic response of muscle to surgical stress in the rat. J Surg Res. 1998; 80.

30. Hankard $\mathrm{RG}$, Haymond $M W$, Darmaun $\mathrm{D}$. Role of glutamine as a glucose precursor in fasting humans. Diabetes. 1997; 40:1535-1541.

31. Stumvoll MW, Perriello $\mathrm{G}$, Nurihan $\mathrm{N}$, et al. Glutamine and alanine metabolism in NIDDM. Diabetes. 1090; 4:863.868. 


\section{CHAPTER 3}

\section{Effects of Surgical Trauma on interorgan}

Glutamine and Amino Acid Metabolism and Organ Protein Kinetics

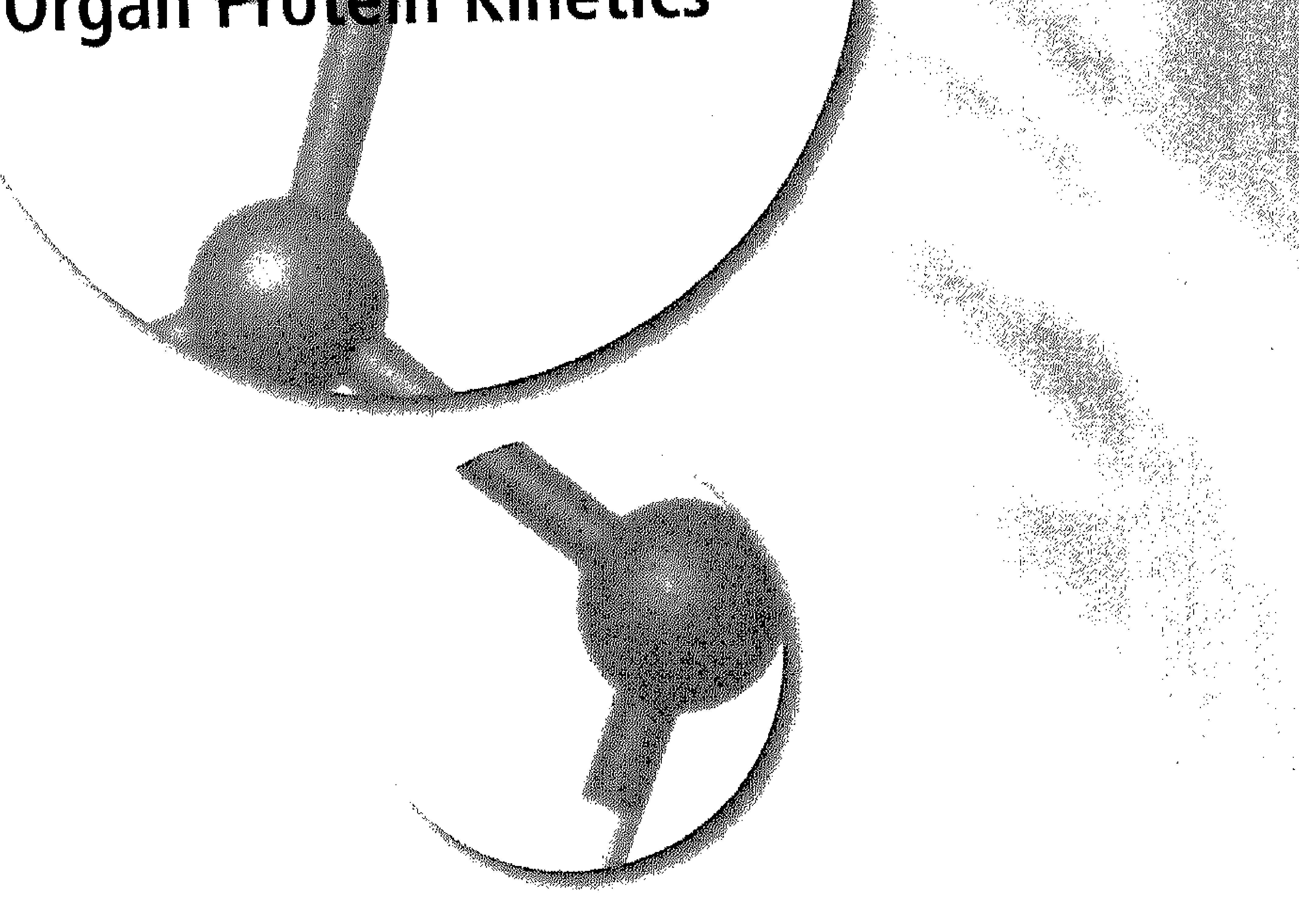

KWE Hulsewé, NEP Deutz 1, AJM Wagenmakers 2, PB Soeters

Department of Surgery, University Hospital Maastricht, The Netherlands, 1 Department of Surgery, Maastricht University, The Netherlands and 2 Department of Human Biology, Maastricht University, The Netherlands 


\section{ABSTRACT}

One of the principal changes occurring in interorgan amino acid metabolism after surgical trauma consists of efflux from glutamine from muscle compartment into the plasma. Since glutamine has a central role in intermediary (aminoacid) metabolism, glutamine supplementation in these circumstances has been tried with differing results. Therefore, we aimed to describe in more detail inter- and intraorgan glutamine kinetics in order to better understand the underlying pathophysiological processes.

In a conscious catheterized pig model we used the arteriovenous (av) balance technique in conjunction with stable isotopes (glutamine, phenylalanine and valine tracers) to determine interorgan amino acid fluxes across the muscle, the portal drained viscera, liver and kidney. Furthermore, using a 2 pool model intraorgan glutamine kinetics were calculated. We compared the postoperative condition (postoperative day 2) with control values obtained after at least 10 days postoperatively.

Compared to control values glutamine plasma concentrations decreased postoperatively. Furthermore, whole body glutamine rate of appearance decreased. Hindquarter glutamine efflux increased although statistically not significant. On the other hand the portal drained viscera took up less glutamine. This was accompanied by a decrease in glutamine disposal and an increase in glutamine production within these organs. The liver changed from glutamine release to glutamine uptake postoperatively, associated with decreased glutamine production and increased glutamine disposal rates. Finally, the kidney increased glutamate uptake after surgery, but glutamine kinetics did not change.

In conclusion, surgical trauma significantly changes glutamine interorgan metabolism. Glutamine uptake by the portal drained viscera decreases and is accompanied by decreased glutamine disposal and increased glutamine production within these organs. On the other hand, the liver consumes glutamine in contrast to control values. The kidney appears not to play an important role in glutamine interorgan metabolism under these conditions. 
Effect of surgical trauma on interorgan glutamine and amino acid metabolism

\section{INTRODUCTION}

The amino acid glutamine plays a pivotal role in amino acid metabolism ${ }^{1}$. Especially under conditions of catabolic stress, glutamine kinetics change significantly. Glutamine is released in large quantities specifically by muscle tissue into the plasma compartment ${ }^{2}$. Plasma and muscle glutamine levels have been shown to decrease in these conditions 3,4 . On the other hand, no changes in whole body glutamine rate of appearance were found after gastrointestinal surgery or in critically ill patients.4,5. This discrepancy possibly can be explained by lower glutamine production rates in other organs, resulting in similar glutamine rates of appearance compared to basal conditions. But what happens with organs which are glutamine consumers? One of the organs most dependent on glutamine as a fuel is the bowel ${ }^{6}$. The gut requires glutamine for a major part of its fuel supply, DNA synthesis, glutathione synthesis and other functions. Previously we have shown that under postoperative conditions plasma glutamine levels decrease and this is accompanied by a decrease in glutamine extraction by the gut in pigs as well as in patients, 7,8 , although increased glutamine concentrations have been reported in intestinal mucosa 3,9 . Clinical deterioration of gut morphology and function has been hypothesized to be the result from glutamine depletion 10 . On the other hand, increased extraction rates by the portal drained viscera have been reported after surgery in dogs ${ }^{11}$. The spleen and the liver also consume glutamine in the postoperative phase $e^{711}$. Since most positive clinical results from glutamine supplementation have been achieved in patient populations enduring severe amounts of stress, e.g. post bone marrow transplantation, after trauma, in critically ill patients etc ${ }^{12-14}$, it is of interest to understand glutamine interorgan metabolism during catabolic stress. Unfortunately, many gaps remain in our understanding of trauma induced changes in interorgan glutamine and amino acid metabolism. For example, the role of the kidney remains largely undefined, as well as changes in glutamine production and consumption and protein breakdown and synthesis rates within different organs. This study was initiated to try to provide more insight in the underlying events in order to explain changes in interorgan amino acid fluxes in the postoperative phase. For this purpose we employed stable isotopes. The main objectives were to quantify glutamine kinetics across the portal drained viscera, the liver and kidneys using a 2 pool model. For reasons explained later, across the hindquarter only arteriovenous balances of glutamine are reported. Furthermore, protein breakdown and synthesis rates in the hindquarter, portal drained viscera and liver were calculated to assess whether the flux of amino acids from muscle tissue is generated by increased protein breakdown or a decrease in synthesis, and simultaneously whether the net uptake of amino acids by the liver is related to increased protein synthesis, decreased protein breakdown or increased oxidation of amino acids, combinations of these metabolic pathways.

\section{MATERIALS AND METHODS}

\section{Animals}

Female crossbred (Yorkshire x Dutch Landrace) piglets aged 10-12 weeks and weighing 18 $22 \mathrm{~kg}$ were used for the study. They were allowed to adapt to the new circumstances for at 
least 1 week in galvanized bar runs ( $2 \times 3$ meter) which were equipped with isolated floors and water nipples. The temperature was held at $22-25^{\circ} \mathrm{C}$ and lights were switched on during 12 hours per day. The pigs were fed with maintenance pellets for adult sows (Landbouwbelang, Roermond, The Netherlands; $16 \%$ raw protein). They had free access to water.

The study was approved of by the Animal Ethics Committee of the Maastricht University.

\section{Surgical procedure}

The evening before surgery an enema (AUV Dierenartsencoöperatie, Cuijk, The Netherlands) was administered to the animals to prevent postoperative ileus. Prior to surgery, the piglets were fasted overnight. Forty-five minutes after intramuscular administration of premedication azoperone (Stresnil ${ }^{\oplus}, 8 \mathrm{mg} / \mathrm{kg}$; Janssen Pharmaceutica, Tilburg, The Netherlands) the piglets were brought under anaesthesia with a mixture of $\mathrm{N} 2 \mathrm{O} / \mathrm{O} 2(1: 2)$ and halothane using a mask. Anaesthesia was maintained using these same volatile anaesthetics. After endotracheal intubation, antibiotic prophylaxis (lincomycin $6.25 \mathrm{mg} / \mathrm{kg}$ and spectinomycin $12.5 \mathrm{mg} / \mathrm{kg}$ ) was given intravenously. Furthermore flunixine (Finadyne, Schering-Plough, Brussels, Belgium) was administered intramuscularly for postoperative analgesia. Fluid resuscitation during surgery consisted of Ringer's lactate (500-1000 ml)

The surgical procedure has been described in part previously 15 and is summarized in figure 1. In short, after a midline laparotomy 8 catheters (Tygon, Norton Co. Akron, OH, USA) were inserted into various blood vessels in order to be able to sample blood across the organs of interest. Two catheters were inserted into the aorta via the left and right deep iliac circumflex arteries. The tip of one catheter was positioned approximately $5 \mathrm{~cm}$

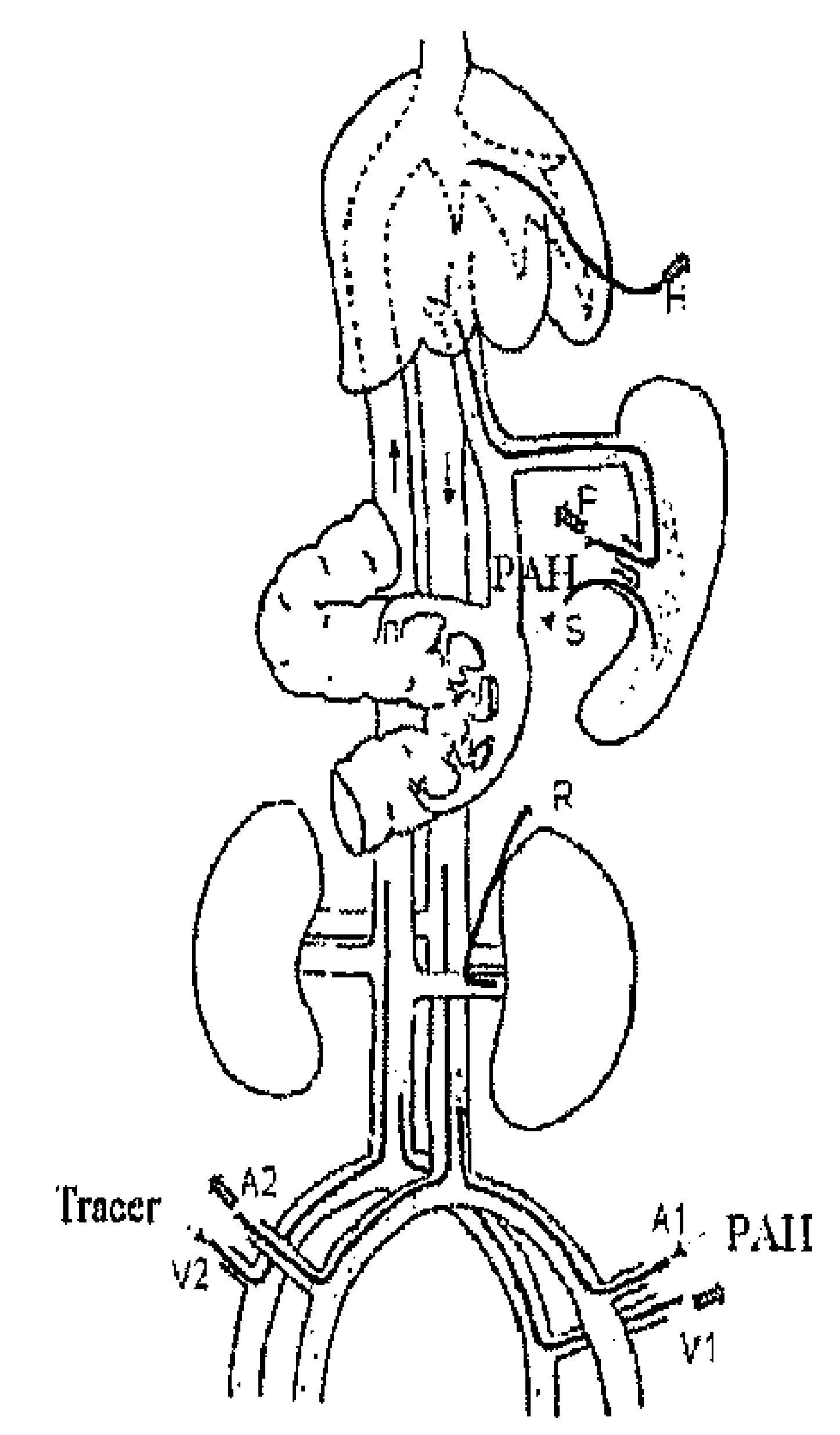

Figure 1 Schematic representation of model used. The arrows indicate centrifugal flow for the arterial system en centripetal flow for venous circulation. PAH represents the sites used for para amino hippuric acid infusion, and tracer indicates site for infusion of the isotopes used. The letters and numbers correspond to the sites as explained in the text. 
Effect of surgical trauma on interorgan glutamine and amino acid metabolism

proximal to the bifurcation (A1), the other above the level of the renal arteries (A2). Similarly two catheters were positioned in the inferior caval vein: one tip lying below (V1) and one above the level of the renal veins (V2). To measure the splanchnic organs, 2 catheters were placed in the splenic vein (S), one of which was positioned with its tip in the portal vein $(\mathrm{P})$ at the liver hilus. One catheter was inserted into the left hepatic vein (H) by transhepatic puncture. Finally after dissection of the left renal hilus, a catheter was positioned into the renal vein (R). After fluoroscopic control of catheter positions, all catheters were secured with sutures and the application of cyanoacrylate. Then a gastrostomy catheter was inserted and secured in place with a purse string suture. All catheters were brought out through a tunnel made through the abdominal wall. The A1 and $S$ catheters were used during the experiments for para amino hippuric acid (PAH) infusion to measure blood flow. The V2 catheter served to infuse stable isotopes during the experiments. The other lines were used for blood sampling.

Finally the abdominal wall was closed in layers. Total operating time was 4-5 hours.

\section{Postoperative care}

After surgery, pigs were allowed to recover in a stable covered with straw. A heating lamp was provided. Each pig wore a canvass harness to protect the catheters and to allow easy handling of the animal. Stomach retention was monitored daily and drained if necessary. Catheters were checked each day for patency, flushed with heparinized saline and filled with a solution containing a mixture of gentamycin and chymotrypsin to prevent infections and obstruction. On the first postoperative day some food was offered. After the first experiment on postoperative day 2 (POD2), the pigs were kept in the runs on straw. They were fed approximately $1 \mathrm{~kg}$ per day of the sow feed. Ten days after surgery the pigs were clinically fully recovered and they were subjected to the same experiment as on POD2.

\section{Tracers}

After baseline blood samples were taken, a primed constant infusion of the following isotopes was started: para-aminohippuric acid (PAH; $25 \mathrm{mM}$, A1422, Sigma Chemical Co.) at a rate of $2 \mathrm{ml} / \mathrm{kg} / \mathrm{h}$ after a 1 hour prime, L-[5.15N] glutamine (prime: $0.78 \mu \mathrm{mol} / \mathrm{kg}$, infusion: $0.78 \mu \mathrm{mol} / \mathrm{kg} / \mathrm{h}$ ), L-[ring ${ }^{2} \mathrm{H} 5$ ] phenylalanine (prime: $3.04 \mu \mathrm{mol} / \mathrm{kg}$, infusion: $3.04 \mu \mathrm{mol} / \mathrm{kg} / \mathrm{h}$ ) and $\mathrm{L}-\left[1-{ }^{13} \mathrm{C}\right]$ valine (prime: $9.6 \mu \mathrm{mol} / \mathrm{kg}$, infusion: $9.6 \mu \mathrm{mol} / \mathrm{kg} / \mathrm{h}$ ). All stable isotopes were purchased from Mass Trace (Woburn, MA, USA) with the exception of the glutamine tracer which was purchased from Cambridge Isotope Laboratories (Woburn, MA, USA).

\section{Experimental protocol}

After an overnight fast, the pigs were transferred to a small movable cage were the experiment was performed under unrestrained, conscious conditions. After sampling of baseline blood samples from the arterial catheter, infusion of PAH was started after administration of a 1-hour bolus through the catheters in the abdominal aorta (A1) and the splenic vein (S). Furthermore, stable isotopes were infused through the catheter V2. After 2 hours when steady state conditions were obtained 16,17 , blood samples were taken from the various catheters. Blood samples for PAH measurements were taken in triplicate. Of all 
blood samples the hematocrit was determined. These experiments were performed on the second postoperative day (POD2) and 10 days after surgery when the animals had recovered from the surgical procedure (CON).

\section{Sample processing}

Immediately after sampling, blood was distributed in heparinized tubes on ice. For determination of PAH $300 \mu \mathrm{L}$ of whole blood was added to $600 \mu \mathrm{L}$ of trichloroacetic acid $(0.7 \mathrm{mmol} / \mathrm{L})$. After thorough mixing and centrifugation, the supernatant was collected for further analysis. Centrifugation was performed at $4^{\circ} \mathrm{C}$ for 5 minutes at $8.500 \mathrm{~g}$ to obtain plasma. For glucose, lactate, ammonia and urea determinations, $900 \mu \mathrm{L}$ plasma was added to $90 \mu \mathrm{L}$ of trichloroacetic acid $(3 \mathrm{mmol} / \mathrm{L})$ to stabilize these metabolites. For amino acid analysis plasma was deproteinized by addition of $500 \mu \mathrm{L}$ plasma to $20 \mathrm{mg}$ dry sulfosalicylic acid. Native plasma was stored for enrichment analysis of the various tracers.

\section{Biochemical analysis}

Glucose, lactate, ammonia, urea and PAH were measured spectrophotometrically on a Cobas Mira S (Roche Diagnostica, Hoffman-La Roche, Basel, Switzerland) by standard enzymatic methods using commercially available kits as described previously 18. Plasma amino acid concentrations were measured on a fully automated High Performance Liquid Chromatography system as described before ${ }^{19,20}$. Isotopic enrichments of phenylalanine and valine were measured on a GC-MS (Finnigan Incos XL, San Jose, CA, USA). Glutamine enrichments were determined on a GC-C-IRMS as described previously ${ }^{21}$. In short, glutamine was extracted from deproteinized plasma samples using an ion-exchange column. The eluted sample was freeze dried and derivatized with $\mathrm{n}$-tert-butyldimethylsilyl-Mmethyl-trifluoracetamide after which the sample was injected into the GC-C-IRMS. Isotopic enrichments are corrected for background (= pre tracer infusion) enrichments and expressed as tracer to tracee ratio's (TTR).

\section{Calculations}

$\alpha$ - Amino nitrogen was calculated as the sum of the individual amino acids measured ${ }^{19}$. Branched chain amino acids were calculated as the sum of leucine, isoleucine and valine concentrations.

Blood flow was measured by measuring the dilution of the indicator PAH ${ }^{15}$.

(1) Flow blood $=\mathrm{I} /([\mathrm{PAH}] \mathrm{v}-[\mathrm{PAH}] \mathrm{a})$

I represents the rate of infusion of $\mathrm{PAH}$. [PAH]v and [PAH]a are the venous and arterial $\mathrm{PAH}$ concentrations respectively.

Blood flow was converted to plasma flow using the following formula:

(2) Flow plasma $=$ flow blood $\times 100 /(100-$ hematocrit $)$

Substrate flux (nmol/ $\mathrm{kg}$ body weight $/ \mathrm{min}$ ) was expressed as the net balance across the specific organ and was calculated by multiplying the plasma flow with the venous $\mathrm{V} / \mathrm{minus}$ 
arterial $[\mathrm{A}]$ substrate concentration (3). A positive flux indicates net release whereas a negative flux reflects net uptake of the substrate across the organ. Splanchnic flux was calculated by multiplying the mean hepatic plasma flow with the hepatic vein - arterial concentration differences. Liver flux was subsequently calculated as the difference of splanchnic minus portal fluxes.

By using tracers protein synthesis and degradation rates in organs can be calculated with the 2-pool model as described by Barrett and Gelfand 22 . The tracer net balance (nb) was calculated in an analogous way to substrate net balance by subtracting the amount of enriched amino acid in the venous compartment from the arterial compartment (4).

(3) $\mathrm{NB}=$ flow * $([\mathrm{V}]-[\mathrm{A}])$

(4) $\mathrm{nb}=$ flow * $\{([\mathrm{A}] *$ TTRa $)-([\mathrm{V}] *$ TTRv $)\}$

$\mathrm{NB}$ and $\mathrm{nb}$ represent the net balance of substrate and tracer respectively. $[\mathrm{V}]$ and $[\mathrm{A}]$ are the venous and arterial substrate concentrations respectively. TTRa refers to the arterial enrichment of the tracee and TTRv to the venous tracee enrichment. The isotopic enrichment in the venous compartment is assumed to represent intracellular enrichment. The rate of disappearance (Rd) reflects loss of the tracer either by incorporation into protein or (oxidative) degradation. Since muscle and intestinal tissues possess little phenylalanine hydroxylase activity, Rd of phenylalanine in these tissues represents protein synthesis. The liver and kidney, however, possess significant phenylalanine hydroxylase activity rendering use of the phenylalanine tracer for protein synthesis determinations unreliable23,24. Valine, on the other hand, appears to be used mainly for protein synthesis in the liver because its degradation rate in the liver is small25. Therefore valine rates of disappearance are used for determination of liver protein synthesis. Because valine is also oxidized by the kidney no calculations of protein synthesis or degradation could be made with regard to the kidney with the tracers used 26 .

(5) $\mathrm{Rd}=\mathrm{nb} / \mathrm{TTRV}$

Since the net balance of substrate across an organ is determined by the difference of production (Ra) and disposal (Rd), the Ra can be calculated using the following formula:

(6) $\mathrm{Ra}=\mathrm{NB}+$ Disposal

Whole body phenylalanine kinetics were calculated as described before by Thompson et al. in formula $(7)^{27}$. Since phenylalanine is an essential amino acid the value obtained represents the whole body rate of protein breakdown.

(7) $\mathrm{Q}=\mathrm{I} / \mathrm{TTRa} 28$

Use of a glutamine tracer gives more insight into glutamine kinetics within the specific organs of interest. The formulas given above can be applied in a similar manner: glutamine Rd represents uptake of glutamine by the organ and glutamine Ra release of glutamine into the venous compartment. Having calculated rates of organ protein synthesis and degradation, 
estimates of glutamine oxidative disposal and de novo glutamine synthesis in the specific organs can be made by subtracting the amounts of glutamine being either incorporated in or released from proteins using the following calculations:

(8) $R d_{\text {gin }} \mathrm{OX}=\mathrm{Rd}_{\mathrm{gln}}-\mathrm{Rd}$ phe/val

(9) $\mathrm{Ra}{ }_{\text {gin }}$ de novo $=\mathrm{Ra}_{\text {gln }}-\mathrm{Ra}$ phe $/$ val

In these formulas, $\mathrm{Rd}$ gin ox represents the amount of glutamine oxidized, $\mathrm{Ra}$ gln $\mathrm{de}$ novo the fraction of glutamine being synthesized de novo. $\mathrm{Rd}_{\mathrm{phe} / \mathrm{val}}$ represents the glutamine used for protein synthesis calculated with phenylalanine tracer (PDV) or valine tracer (liver). Ra phe/val indicates glutamine derived from protein breakdown calculated with phenylalanine (PDV) or valine tracers (liver). For these calculations the glutamine content of protein was estimated to be $6.95 \mathrm{~g} / 100 \mathrm{~g}$ protein 29 . Using the 2 pool model, it is assumed that an isotopic steady state of the tracer within the organ of interest exists. Since it is known that glutamine tracer does not equilibrate readily with the very large muscle glutamine pool, no glutamine kinetics across the hindquarter are calculated ${ }^{21}$.

\section{Statistics}

Results are presented as means \pm standard errors of the mean (SEM). Differences between groups were tested with the Wilcoxon test, if sufficient paired observations were available, or otherwise the Mann Whitney U test, for significance. A p value of $<0.05$ was considered to be significant.

\section{RESULTS}

In total 11 pigs were included in the POD2 group and 11 pigs in the CON group. Due to loss of catheter patency on average 9 observations on amino acid flux per group were available for all organs with the exception of the renal fluxes in the CON group in which there were 6 observations.

Table $1 \quad$ Plasma flows across hindquarter, PDV, liver and kidneys (ml/kg body weight/min)

\begin{tabular}{llll}
\hline & CON $(n=11)$ & POD2 $(n=11)$ & P \\
\hline Hindquarter & $21.4 \pm 2.8$ & $13.9 \pm 1.8$ & NS $(0.07)$ \\
PDV & $36.8 \pm 8.3$ & $29.6 \pm 4.3$ & NS \\
Liver & $41.1 \pm 7.3$ & $45.8 \pm 6.1$ & NS \\
Kidney & $39.8 \pm 13.5$ & $22.2 \pm 2.8$ & NS \\
\hline
\end{tabular}

PDV denotes the portal drained viscera and the table shows plasma flows across the respective organs as means \pm SEM. Values are expressed in $\mathrm{ml} / \mathrm{kg}$ body weight/min.

CON is the Control group and POD2 represents the postoperative group. 
Effect of surgical trauma on interorgan glutamine and amino acid metabolism

\section{Plasma flow}

Plasma flows across the hindquarter, PDV, liver and kidneys are summarized in table 1. No significant difference in flow across any organ was detected.

\section{Arterial concentrations (Table 2)}

Glutamine concentrations in the POD2 group were lower than CON values: $358 \pm 77$ and

Table 2 Arterial amino acid and substrate concentrations ( $\mu$ mol/L)

\begin{tabular}{|c|c|c|c|c|c|c|c|}
\hline \multirow[b]{2}{*}{ Glutamate } & \multicolumn{3}{|c|}{ CON } & \multicolumn{3}{|c|}{ POD2 } & \multirow[t]{2}{*}{$\mathbf{P}$} \\
\hline & 238 & \pm & 35 & 263 & \pm & 43 & \\
\hline Asparagine & 26 & \pm & 5 & 33 & \pm & 4 & \\
\hline Serine & 99 & \pm & 9 & 106 & \pm & 8 & \\
\hline Glutamine & 358 & \pm & 77 & 473 & \pm & 102 & 0.028 \\
\hline Glycine & 422 & \pm & 60 & 675 & \pm & 86 & 0.018 \\
\hline Histidine & 79 & \pm & 14 & 87 & \pm & 15 & \\
\hline Threonine & 48 & \pm & 3 & 53 & \pm & 3 & \\
\hline Citrulline & 55 & \pm & 6 & 53 & \pm & 4 & \\
\hline Alanine & 145 & \pm & 27 & 248 & \pm & 37 & \\
\hline Arginine & 77 & \pm & 8 & 68 & \pm & 7 & \\
\hline Taurine & 65 & & 4 & 72 & \pm & 5 & \\
\hline Alpha-aminobutyric acid & 77 & \pm & 13 & 11 & \pm & 4 & 0.018 \\
\hline Tyrosine & 32 & \pm & 3 & 32 & \pm & 4 & \\
\hline Valine & 243 & \pm & 24 & 198 & \pm & 22 & 0.028 \\
\hline Methionine & 22 & \pm & 4 & 29 & \pm & 6 & 0.018 \\
\hline Isoleucine & 162 & \pm & 9 & 105 & \pm & 6 & 0.018 \\
\hline Phenylalanine & 76 & \pm & 6 & 59 & \pm & 5 & \\
\hline Tryptophane & 12 & \pm & 2 & 15 & \pm & 1 & \\
\hline Leucine & 149 & \pm & 12 & 99 & \pm & 7 & 0.018 \\
\hline Ornithine & 52 & \pm & 5 & 53 & \pm & 8 & \\
\hline Lysine & 105 & \pm & 8 & 94 & \pm & 14 & \\
\hline Branched chain amino acids & 554 & \pm & 35 & 402 & \pm & 26 & 0.018 \\
\hline \multirow[t]{2}{*}{ Sum of all amino acids } & 2542 & \pm & 76 & 2812 & \pm & 204 & \\
\hline & \multicolumn{3}{|c|}{$\overline{\mathrm{CON}}$} & \multicolumn{3}{|c|}{ POD2 } & $P$ \\
\hline Glucose & 4422 & $\overline{ \pm}$ & 125 & 4880 & \pm & 155 & 0.04 \\
\hline Lactate & 615 & \pm & 83 & 642 & \pm & 121 & \\
\hline Ammonia & 80.0 & \pm & 6.2 & 73.1 & \pm & 6.1 & \\
\hline Urea & 2875 & \pm & 704 & 3450 & \pm & 351 & \\
\hline
\end{tabular}

Arterial amino acid and substrate concentrations expressed as means \pm SEM. CON is the control group and POD2 represents the postoperative group,

Values are expressed in $\mu \mathrm{mol} / \mathrm{L}$ 
$473 \pm 102 \mu \mathrm{mol} / \mathrm{L}$ ( $p=0.03$ ) respectively. Furthermore, all branched chain amino acids were significantly lower, individually as well summated. The decrease was $22 \%$ for valine $(\mathrm{p}=0.03$ ) and approximately $50 \%$ for leucine and isoleucine ( $\mathrm{p}=0.02$ and 0.02 respectively). However, the concentration of total $\alpha$ - amino nitrogen did not change significantly.

\section{Whole body appearances}

No changes in whole body appearance rates of phenylalanine and valine were observed. In contrast, glutamine whole body rate of appearance was $5823 \pm 451$ in the CON group and significantly lower in the POD2 group (4732 $\pm 221 \mathrm{nmol} / \mathrm{kg} / \mathrm{min},(\mathrm{p}=0.03)$. These data are summarized in figure 2 .

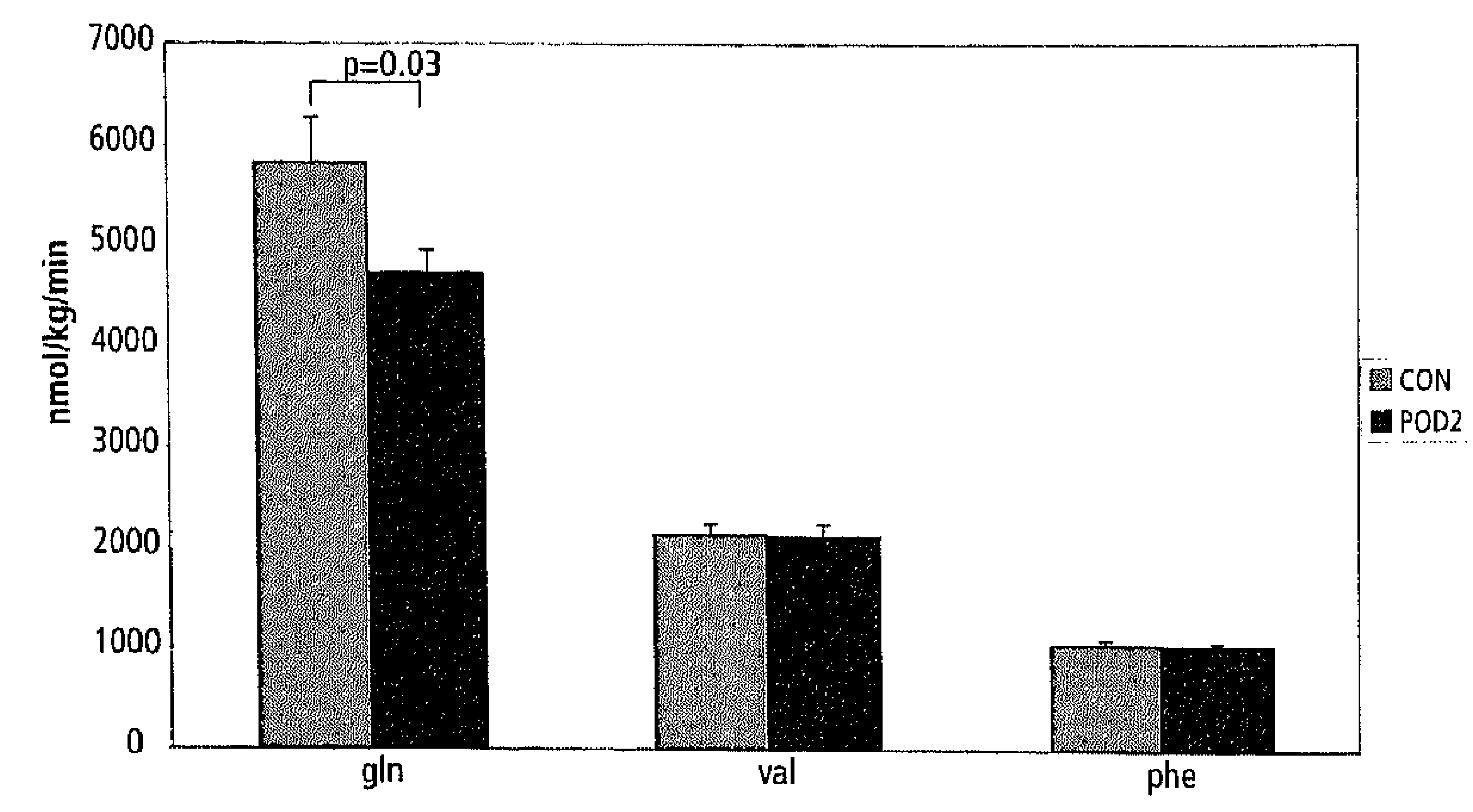

Figure 2 Effects of surgical trauma on whole body rate of appearance (WbRa) of phenylalanine (phe), valine (val) and glutamine (gln). CON is the control group and POD2 represents the postoperative group. Values are expressed in nmol $/ \mathrm{kg} / \mathrm{min}$.

\section{Hindquarter (Table 3)}

Glucose and lactate fluxes across the hindquarter were not affected by the surgery. Glutamine efflux from the hindquarter almost doubled from $1000 \pm 285$ to $1854 \pm 409$ $\mathrm{nmol} / \mathrm{kg} / \mathrm{min}$ but did not reach statistical significance. Alanine efflux increased even more than tenfold $(p=0.007)$. The branched chain amino acids were also released in significantly higher amounts after trauma.

Total $\alpha$ - amino nitrogen efflux from the hindquarter changed from approximately zero balance to $7455 \pm 2249 \mathrm{nmol} / \mathrm{kg} / \mathrm{min}(\mathrm{p}=0.004)$.

Phenylalanine disposal decreased from $241 \pm 57$ to $181 \pm 70 \mathrm{nmol} / \mathrm{kg} / \mathrm{min}$ but this was not significant. Phenylalanine production tended to increase from $336 \pm 62$ to $533 \pm$ $111 \mathrm{nmol} / \mathrm{kg} / \mathrm{min}(\mathrm{p}=0.09$ ) (figure 3).

\section{Portal Drained Viscera (Table 4)}

Glucose and lactate balances across the PDV did not change significantly. Glutamine uptake 
Effect of surgical trauma on interorgan glutamine and amino acid metabolism

Table 3 Muscle amino acid and substrate fluxes in nmol/ $\mathrm{kg} / \mathrm{min}$

\begin{tabular}{|c|c|c|c|c|c|c|c|}
\hline & & $\mathrm{CON}$ & & & OD: & & $\bar{p}$ \\
\hline Glutamate & -1756 & \pm & 251 & -1033 & \pm & 175 & 0.031 \\
\hline Asparagine & 33 & \pm & 28 & 280 & \pm & 90 & 0.014 \\
\hline Serine & -84 & \pm & 113 & 228 & \pm & 115 & 0.031 \\
\hline Glutamine & 1000 & \pm & 285 & 1854 & \pm & 409 & \\
\hline Glycine & -21 & \pm & 269 & 714 & \pm & 197 & 0.059 \\
\hline Histidine & 9 & \pm & 131 & 528 & \pm & 154 & 0.014 \\
\hline Threonine & 95 & \pm & 63 & 221 & \pm & 55 & \\
\hline Citrulline & -60 & \pm & 46 & 1 & \pm & 9 & \\
\hline Alanine & 178 & \pm & 191 & 1855 & \pm & 587 & 0.006 \\
\hline Arginine & 111 & \pm & 133 & 3 & \pm & 12 & \\
\hline Taurine & -18 & & 38 & 296 & \pm & 111 & 0.014 \\
\hline Alpha-aminobutyric acid & 37 & \pm & 29 & 17 & \pm & 14 & \\
\hline Tyrosine & 31 & \pm & 23 & 207 & \pm & 74 & 0.077 \\
\hline Valine & 15 & \pm & 40 & 351 & \pm & 94 & 0.006 \\
\hline Methionine & 103 & \pm & 58 & 184 & \pm & 54 & \\
\hline Isoleucine & 24 & \pm & 50 & 240 & \pm & 81 & 0.027 \\
\hline Phenylalanine & 56 & \pm & 27 & 323 & \pm & 92 & 0.024 \\
\hline Tryptophane & 11 & \pm & 33 & 66 & \pm & 20 & \\
\hline Leucine & 99 & \pm & 94 & 509 & \pm & 130 & 0.014 \\
\hline Ornithine & 18 & \pm & 87 & -17 & \pm & 10 & \\
\hline Lysine & 99 & \pm & 75 & 629 & \pm & 177 & 0.008 \\
\hline Branched chain amino acids & 109 & \pm & 132 & 1100 & \pm & 290 & 0.008 \\
\hline Sum of all amino acids & -34 & \pm & 909 & 7455 & \pm & 2249 & 0.004 \\
\hline
\end{tabular}

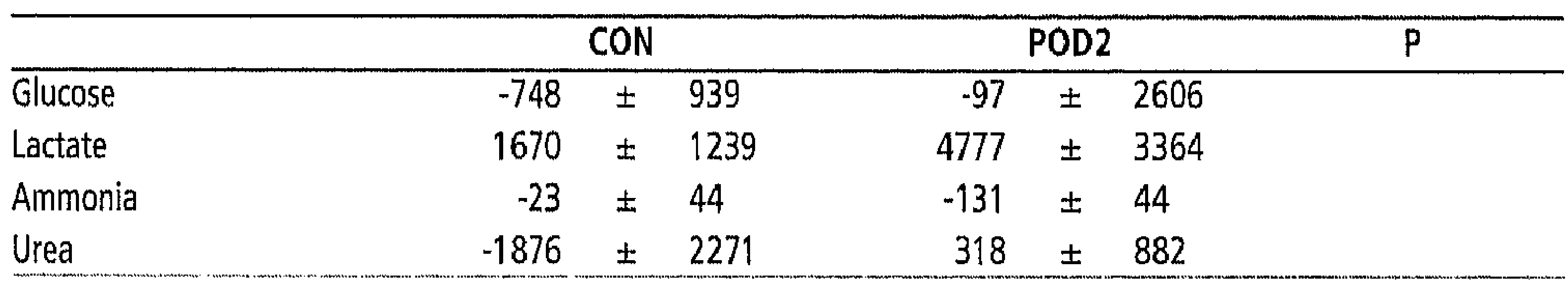

Amino acid and substrate fluxes across the hindquarter expressed as means \pm SEM. CON is the control group and POD2 represents the postoperative group.

Values are expressed in $\mathrm{nmol} / \mathrm{kg} / \mathrm{min}$.

decreased on POD2 in parallel with the decreased arterial glutamine levels but failed to reach statistical significance $(p=0.07)$. Alanine release increased from $12 \pm 151$ to $260 \pm$ $113 \mathrm{nmol} / \mathrm{kg} / \mathrm{min}$. Balances of other amino acids across the PDV were neither substantially nor significantly affected by surgery.

Protein breakdown represented by phenylalanine production tended to decrease on 

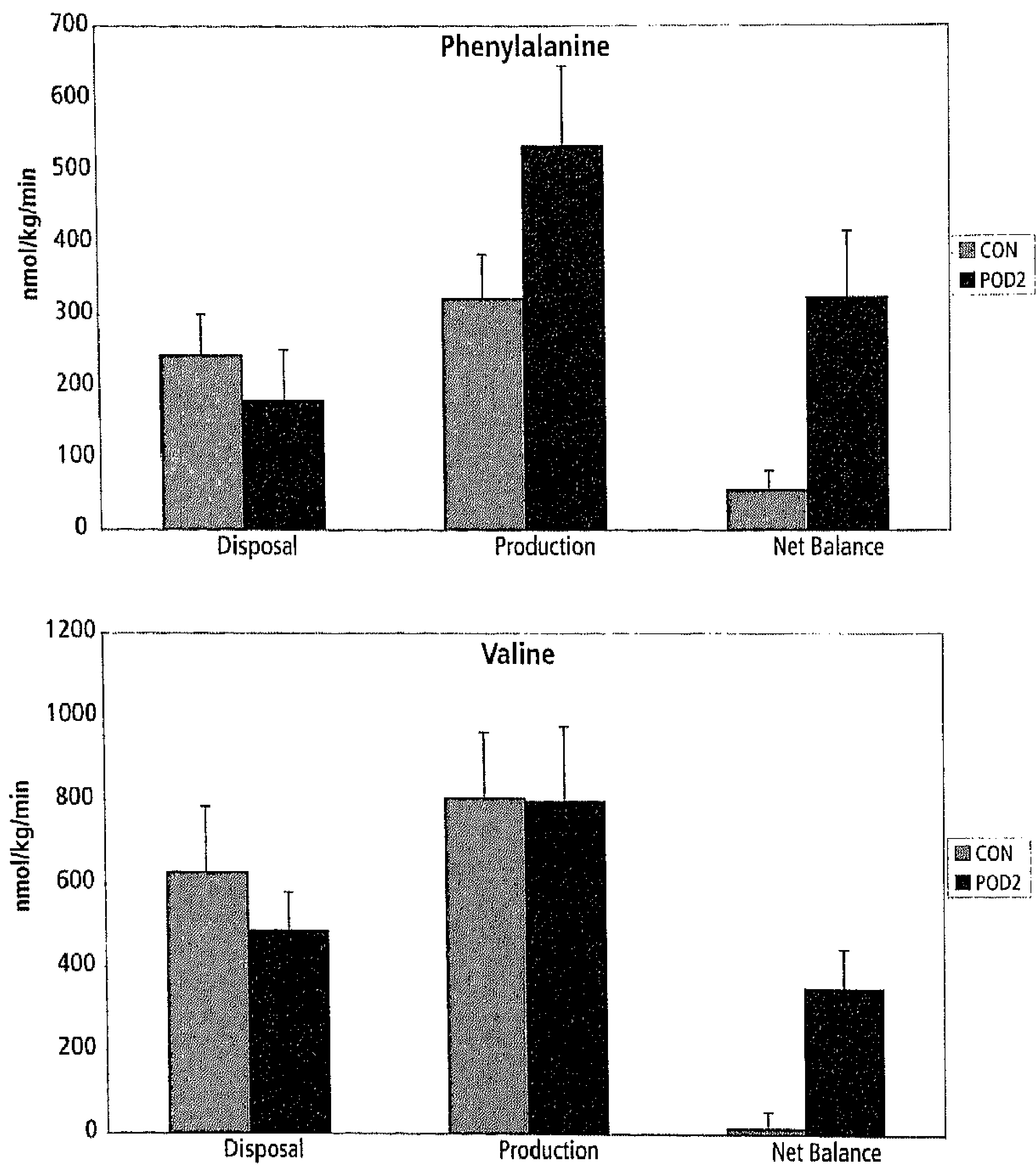

Figure 3 Effects of surgical trauma on whole body rate of appearance (WbRa) of phenylalanine (phe), valine (val) and glutamine (gln). CON is the control group and POD2 represents the postoperative group. Values are expressed in $\mathrm{nmol} / \mathrm{kg} / \mathrm{min}$.

POD2 but failed to reach significance ( $561 \pm 31$ versus $448 \pm 74 \mathrm{nmol} / \mathrm{kg} / \mathrm{min}, \mathrm{p}=0.09$ ). Glutamine production tended to increase $(892 \pm 117$ versus $1193 \pm 222 \mathrm{nmol} / \mathrm{kg} / \mathrm{min}$, $\mathrm{p}=0.07)$. Glutamine disposal in the PDV decreased significantly on POD2 $(3183 \pm 163$ versus $2161 \pm 345 \mathrm{nmol} / \mathrm{kg} / \mathrm{min}, \mathrm{p}=0.021$. Protein and glutamine kinetics are summarized in figure 4. Simultaneously, a significant decrease in glutamine oxidation rates was observed within the portal drained viscera (table 7). Relative to glutamine Rd, however, oxidation rates remained similar. De novo synthesis rates of glutamine, however, increased significantly after surgery. 
Effect of surgical trauma on interorgan glutamine and amino acid metabolism

Table $4 \quad$ PDV fluxes

\begin{tabular}{|c|c|c|c|c|c|c|c|}
\hline \multirow[b]{2}{*}{ Glutamate } & \multicolumn{3}{|c|}{ CON } & \multicolumn{3}{|c|}{ POD2 } & \multirow[t]{2}{*}{$\mathbf{P}$} \\
\hline & -848 & \pm & 316 & -692 & \pm & 259 & \\
\hline Asparagine & 8 & \pm & 44 & .7 & \pm & 76 & \\
\hline Serine & -399 & \pm & 84 & -439 & \pm & 101 & \\
\hline Glutamine & -2190 & \pm & 288 & -1582 & \pm & 472 & 0.07 \\
\hline Glycine & 452 & \pm & 538 & 244 & \pm & 124 & \\
\hline Histidine & -219 & \pm & 232 & -294 & \pm & 110 & \\
\hline Threonine & -55 & \pm & 36 & -30 & \pm & 23 & \\
\hline Citrulline & 238 & \pm & 51 & 370 & \pm & 83 & \\
\hline Alanine & 12 & \pm & 151 & 260 & \pm & 113 & \\
\hline Arginine & 17 & \pm & 75 & 14 & \pm & 25 & \\
\hline Taurine & -53 & \pm & 73 & -60 & \pm & 53 & \\
\hline Alpha-aminobutyric acid & 57 & \pm & 55 & 3 & \pm & 19 & \\
\hline Tyrosine & 1 & \pm & 36 & -55 & \pm & 48 & \\
\hline Valine & -313 & \pm & 219 & -176 & \pm & 92 & \\
\hline Methionine & -40 & \pm & 42 & -73 & \pm & 24 & \\
\hline Isoleucine & -122 & \pm & 75 & -136 & \pm & 63 & \\
\hline Phenylalanine & 40 & \pm & 68 & -19 & \pm & 45 & \\
\hline Tryptophane & -29 & \pm & 18 & -23 & \pm & 25 & \\
\hline Leucine & -99 & \pm & 95 & -162 & \pm & 122 & \\
\hline Ornithine & 153 & \pm & 217 & -178 & \pm & 61 & \\
\hline Lysine & -81 & \pm & 129 & -5 & \pm & 72 & \\
\hline Branched chain amino acids & -531 & \pm & 362 & .474 & \pm & 270 & \\
\hline Sum of all amino acids & -3110 & \pm & 1090 & -2941 & \pm & 1073 & \\
\hline
\end{tabular}

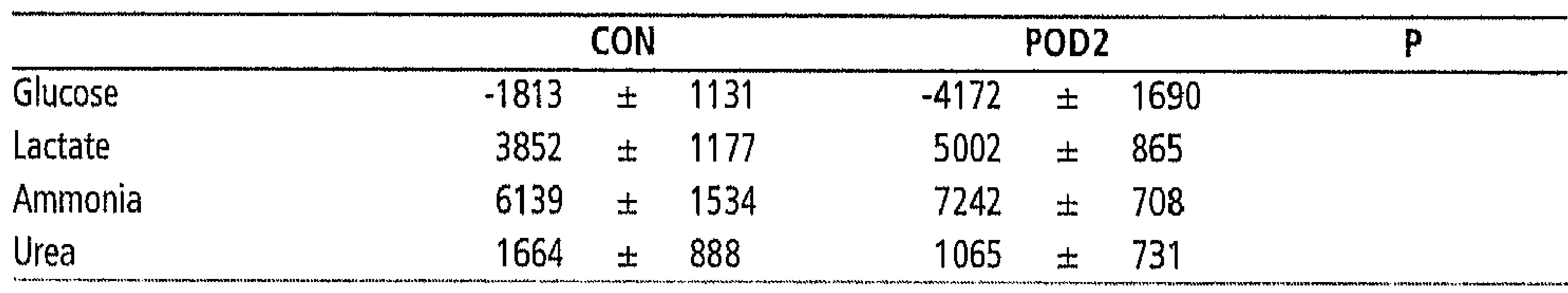

Amino acid and substrate fluxes across the portal drained viscera expressed as means \pm SEM. CON is the control group and POD2 represents the postoperative group.

Values are expressed in nmol/ $\mathrm{kg} / \mathrm{min}$.

\section{Liver (Table 5)}

Fluxes of glucose, lactate, ammonia and urea were not significantly different on POD2. Glutamine flux changed from a net release to uptake (CON $851 \pm 597$ versus POD2 -1923 $\pm 672 \mathrm{nmol} / \mathrm{kg} / \mathrm{min}, \mathrm{p}=0.01$ ). In the control experiment total branched chain amino acid showed slight efflux whereas on POD2 net uptake by the liver was observed. This change 

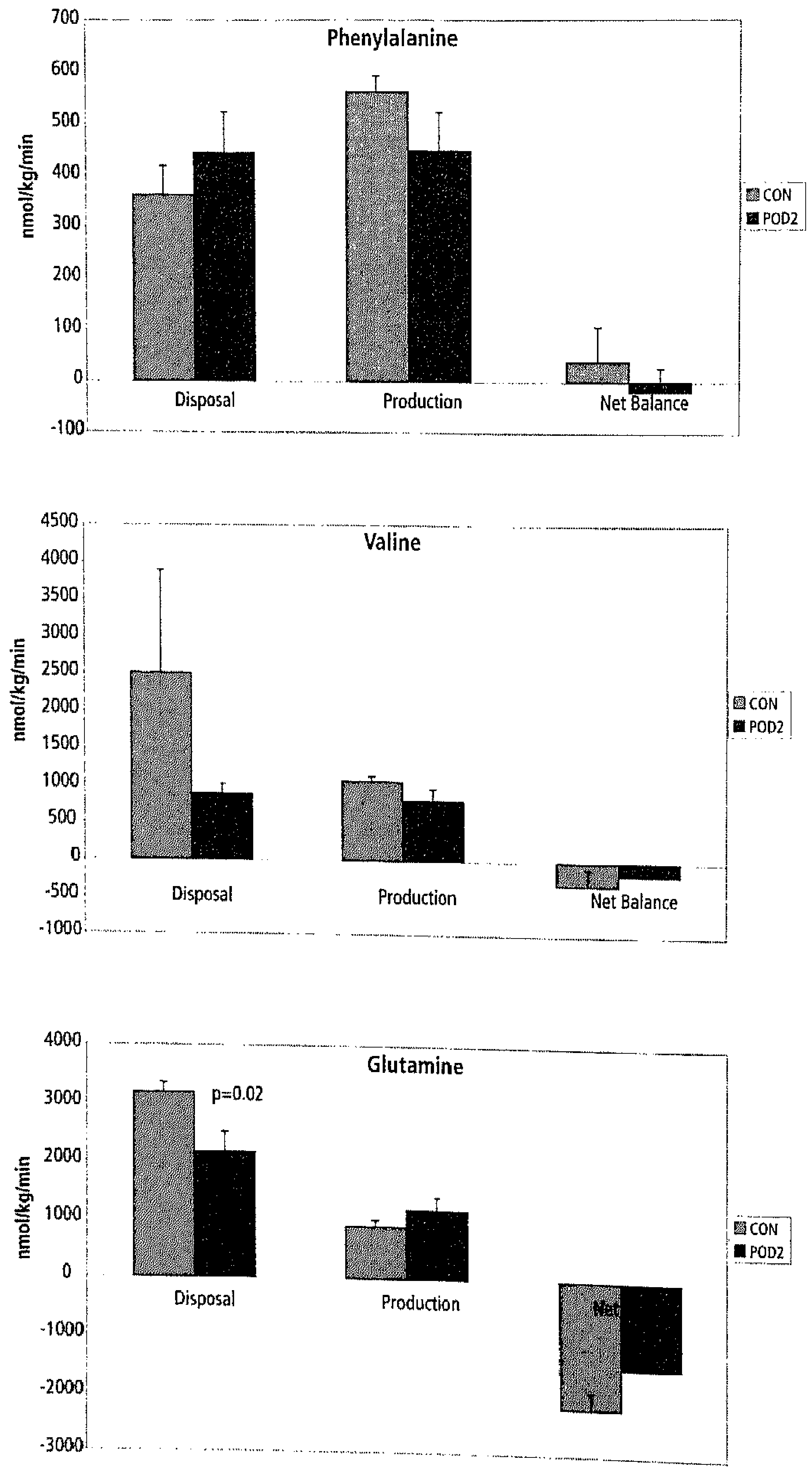

Figure 4 Thenylalanine, valine and glutamine kinetics across portal drained viscera. CON is the control group and POID? represents the postoperative group. Values are expressed in nmol/ $\mathrm{kg} / \mathrm{min}$. 
however did not reach significance $(p=0.09)$. Similarly, total $\alpha$ - amino nitrogen changed from approximately zero balance to net uptake without statistical significance.

Valine kinetics showed no changes. The shift from glutamine production to uptake resulted from a combined increased glutamine disposal rate and a decrease in glutamine production by the liver although these changes separately were not statistically significant The tracer data are summarized in figure 5. Similarly, glutamine de novo synthesis and oxidation rates, both in absolute figures and relative to total glutamine $\mathrm{Ra}$ and $\mathrm{Rd}$, remained unchanged (table 7).
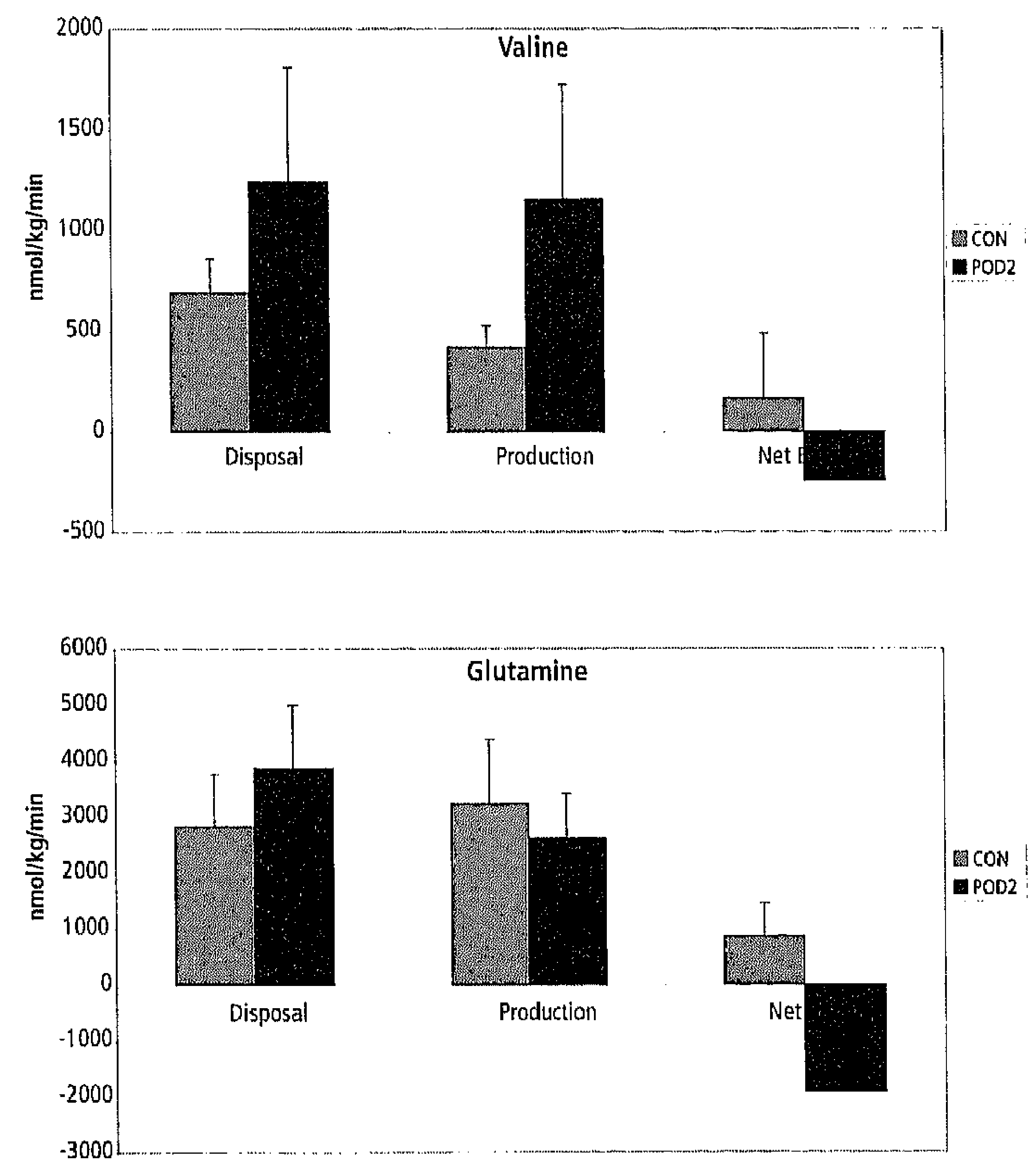

Figure $5 \quad$ Phenylalanine, valine and glutamine kinetics across the liver. CON is the control group and POD2 represents the postoperative group. Values are expressed in $\mathrm{nmol} / \mathrm{kg} / \mathrm{min}$. 
Table 5 Hepatic fluxes

\begin{tabular}{lrlllll}
\hline & \multicolumn{3}{c}{ CON } & \multicolumn{2}{l}{ POD2 } & P \\
\hline Glutamate & 4005 & \pm 1228 & 3962 & \pm 477 & \\
Asparagine & -140 & \pm 58 & -453 & \pm 119 & 0.011 \\
Serine & 403 & \pm 148 & -434 & \pm 195 & 0.002 \\
Glutamine & 851 & \pm 597 & -1923 & \pm 672 & 0.008 \\
Glycine & -1776 & \pm 555 & -3180 & \pm 635 & \\
Histidine & -506 & \pm 507 & -515 & \pm 255 & \\
Threonine & -69 & \pm 75 & -400 & \pm 81 & 0.008 \\
Citrulline & -161 & \pm 103 & -226 & \pm 76 & \\
Alanine & -1294 & \pm 287 & -2916 & \pm 734 & 0.091 \\
Arginine & -372 & \pm 388 & 42 & \pm 24 & \\
Taurine & -81 & \pm 90 & -599 & \pm 199 & 0.004 \\
Alpha-aminobutyric acid & -26 & \pm 63 & 32 & \pm 88 & \\
Tyrosine & -177 & \pm 73 & -409 & \pm 120 & \\
Valine & 164 & \pm 322 & -245 & \pm 145 & \\
Methionine & -26 & \pm 84 & -255 & \pm 149 & \\
Isoleucine & 12 & \pm 118 & -318 & \pm 126 & 0.054 \\
Phenylalanine & -144 & \pm 93 & -395 & \pm 172 & \\
Tryptophane & -39 & \pm 72 & -94 & \pm 35 & \\
Leucine & -51 & \pm 131 & -483 & \pm 156 & 0.050 \\
Ornithine & -174 & \pm 247 & 199 & \pm 73 & \\
Lysine & -97 & \pm 188 & -1037 & \pm 403 & 0.021 \\
Branched chain amino acids & 105 & \pm 550 & -1046 & \pm 391 & 0.050 \\
Sum of all amino acids & 181 & \pm 2408 & -10230 & \pm 4034 & 0.065 \\
\hline
\end{tabular}

\begin{tabular}{|c|c|c|c|c|c|c|c|}
\hline & \multicolumn{3}{|c|}{$\mathrm{CON}$} & \multicolumn{3}{|c|}{ POD2 } & $\bar{P}$ \\
\hline Glucose & 13999 & \pm & 3217 & 16846 & $\overline{ \pm}$ & 10050 & \\
\hline Lactate & 13903 & \pm & 2377 & 10283 & \pm & 5057 & \\
\hline Ammonia & -7674 & \pm & 1590 & -7165 & \pm & 785 & \\
\hline Urea & 912 & \pm & 717 & 312 & \pm & $\$ 113$ & \\
\hline
\end{tabular}

Amino acid and substrate fluxes across the liver expressed as means \pm SEM. CON is the control group and POD2 represents the postoperative group.

Values are expressed in nmol $/ \mathrm{kg} / \mathrm{min}$.

\section{Kidneys (Table 6)}

Data from renal AV measurements were characterized by relatively large variations. Glutamate uptake by the kidneys decreased significantly on POD2 (-982 \pm 300 versus $-4427 \pm 1185$ $\mathrm{nmol} / \mathrm{kg} / \mathrm{min}, \mathrm{p}<0.03$ ). Uptake of glutamine and arginine decreased but not significantly. 
Renal citrulline fluxes remained unchanged. Furthermore, no changes in total $\alpha$ - amino nitrogen flux was observed (Table 6). Glutamine kinetics remained similar (figure 6).

Table 6 Renal fluxes

\begin{tabular}{|c|c|c|c|c|c|c|c|}
\hline \multirow[b]{2}{*}{ Glutamate } & \multicolumn{3}{|c|}{$\mathrm{CON}$} & \multicolumn{3}{|c|}{ POD2 } & \multirow{2}{*}{$\frac{\mathbf{P}}{0.03}$} \\
\hline & -4427 & \pm & 1185 & -982 & \pm & 300 & \\
\hline Asparagine & 19 & \pm & 52 & 102 & \pm & 35 & \\
\hline Serine & 1397 & \pm & 336 & 674 & \pm & 140 & \\
\hline Glutamine & -4543 & \pm & 4214 & -237 & \pm & 421 & \\
\hline Glycine & -683 & \pm & 1326 & 679 & \pm & 209 & \\
\hline Histidine & 1562 & \pm & 1055 & 316 & \pm & 125 & \\
\hline Threonine & 619 & \pm & 538 & 144 & \pm & 47 & \\
\hline Citrulline & -637 & \pm & 208 & -345 & \pm & 59 & \\
\hline Alanine & -276 & \pm & 119 & -38 & \pm & 305 & \\
\hline Arginine & -359 & \pm & 352 & -10 & \pm & 39 & \\
\hline Taurine & 1174 & \pm & 916 & 186 & \pm & 43 & \\
\hline Alpha-aminobutyric acid & 156 & \pm & 227 & -4 & \pm & 50 & \\
\hline Tyrosine & 131 & \pm & 43 & 90 & \pm & 34 & \\
\hline Valine & 2163 & \pm & 2033 & 180 & \pm & 97 & \\
\hline Methionine & 753 & \pm & 759 & 82 & \pm & 33 & \\
\hline Isoleucine & 334 & \pm & 236 & 55 & \pm & 84 & \\
\hline Phenylalanine & 954 & \pm & 776 & 165 & \pm & 79 & \\
\hline Tryptophane & 295 & \pm & 232 & 23 & \pm & 10 & \\
\hline Leucine & 679 & \pm & 466 & 252 & \pm & 91 & \\
\hline Ornithine & 705 & \pm & 396 & 212 & \pm & 63 & \\
\hline Lysine & 1824 & \pm & 1367 & 246 & \pm & 109 & \\
\hline Branched chain amino acids & 2894 & \pm & 2756 & 488 & \pm & 244 & \\
\hline Sum of all amino acids & 1815 & \pm & 3244 & 1789 & \pm & 1263 & \\
\hline
\end{tabular}

Amino acid and substrate fluxes across the kidneys expressed as means \pm SEM. CON is the control group and POD2 represents the postoperative group.

Values are expressed in $\mathrm{nmol} / \mathrm{kg} / \mathrm{min}$. 


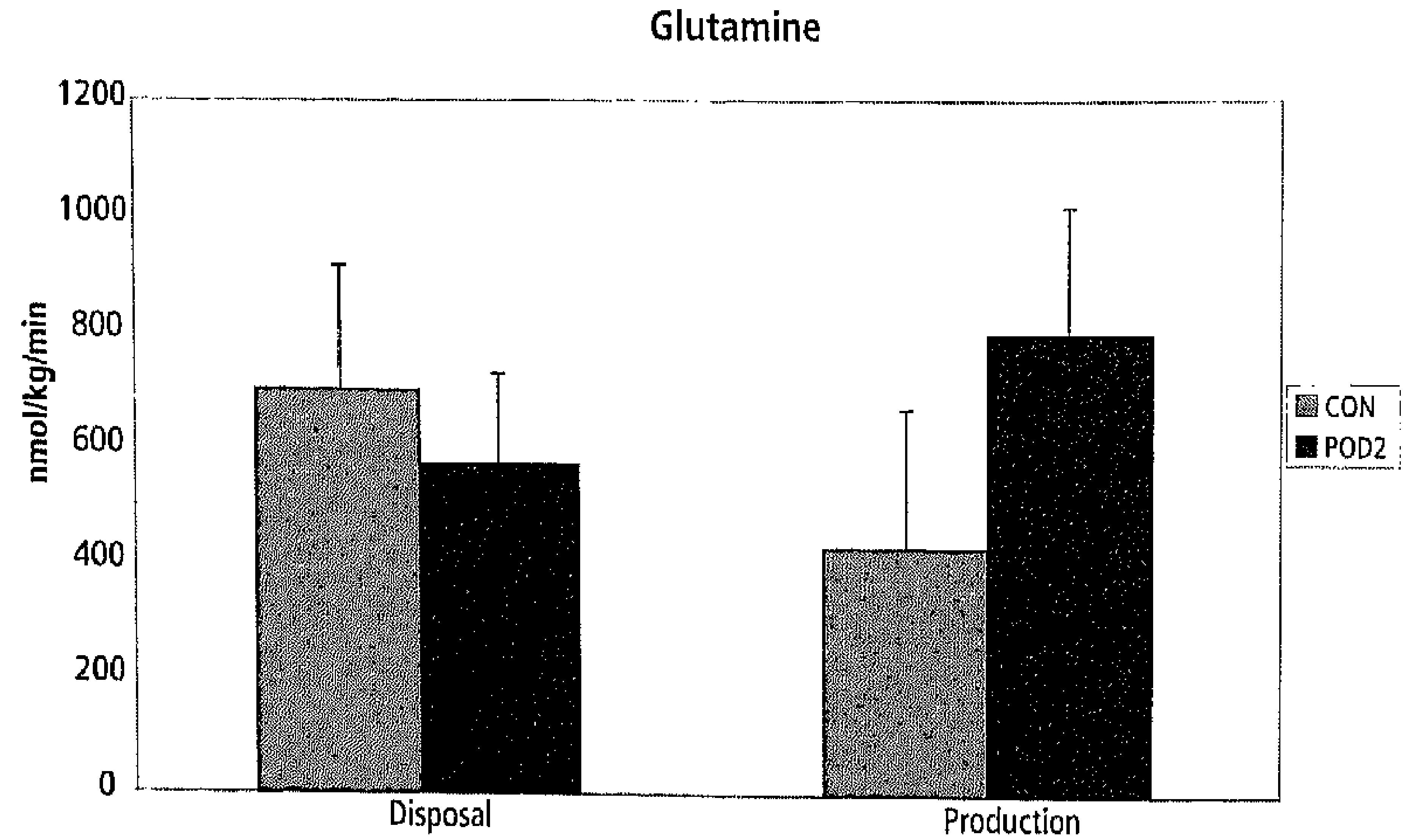

Figure 6 Glutamine kinetics across the kidneys. CON is the control group and POD2 represents the postoperative group. Values are expressed in nmol $/ \mathrm{kg} / \mathrm{min}$.
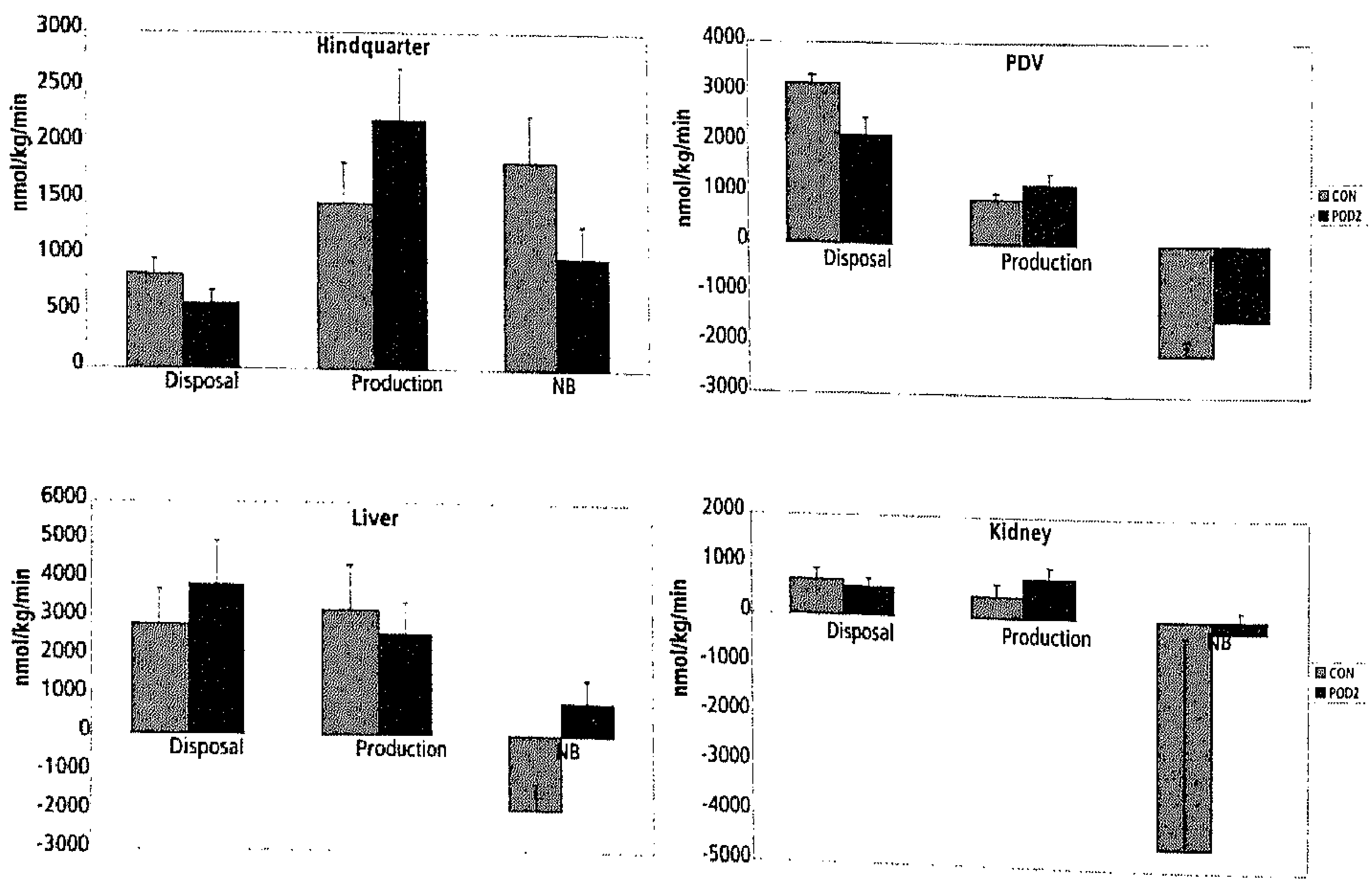

Figure 7 Glutamine disposal, production and (substrate) Net Balance (NB) across the hindquarter, portal drained viscera (FDV), liver and kidney. CON is the control group and POD2 represents the postoperative group. Values are expressed in $\mathrm{nmol} / \mathrm{kg} / \mathrm{min}$. 
Effect of surgical trauma on interorgan glutamine and amino acid metabolism

Table $7 \quad$ Glutamine de novo synthesis and oxidation rates in the PDV and liver

\begin{tabular}{|c|c|c|c|c|}
\hline & & & $\begin{array}{l}\text { De novo } \\
\text { synthesis }\end{array}$ & Oxidation \\
\hline \multirow[t]{4}{*}{$\overline{\mathrm{PDV}}$} & & POD2 & $684 \pm 129$ & $1685 \pm 258$ \\
\hline & $\%$ of $R a / R d$ & & $50 \pm 7$ & $86 \pm 7$ \\
\hline & & $\mathrm{CON}$ & $187 \pm 57$ & $2565 \pm 236$ \\
\hline & $\%$ of $\mathrm{Ra} / \mathrm{Rd}$ & & $18 \pm 5$ & $87 \pm 4$ \\
\hline \multirow[t]{4}{*}{ Liver } & & POD2 & $1604 \pm 891$ & $4912 \pm 2106$ \\
\hline & $\%$ of $\mathrm{Ra} / \mathrm{Rd}$ & & $36 \pm 20$ & $82 \pm 6$ \\
\hline & & CON & $1697 \pm 643$ & $2420 \pm 958$ \\
\hline & $\%$ of $\mathrm{Ra} / \mathrm{Rd}$ & & $66 \pm 13$ & $71 \pm 20$ \\
\hline
\end{tabular}

Glutamine de novo synthesis and oxidation rates in the portal drained viscera and liver expressed as means \pm SEM. CON is the control group and POD2 represents the postoperative group.

Values are expressed in nmol/kg/min or as a percentage of the Ra or Rd respectively.

For an overview organ glutamine and protein kinetics are summarized in figure 7 and 8 respectively.
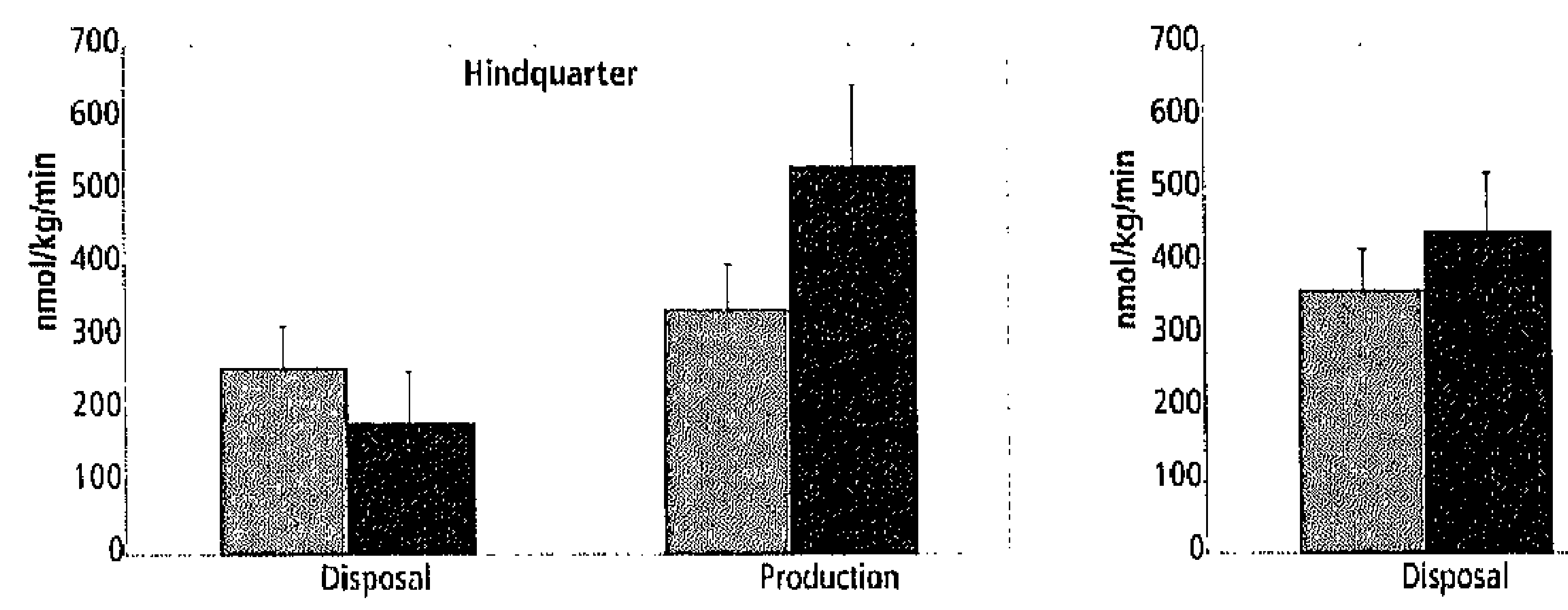

PDV

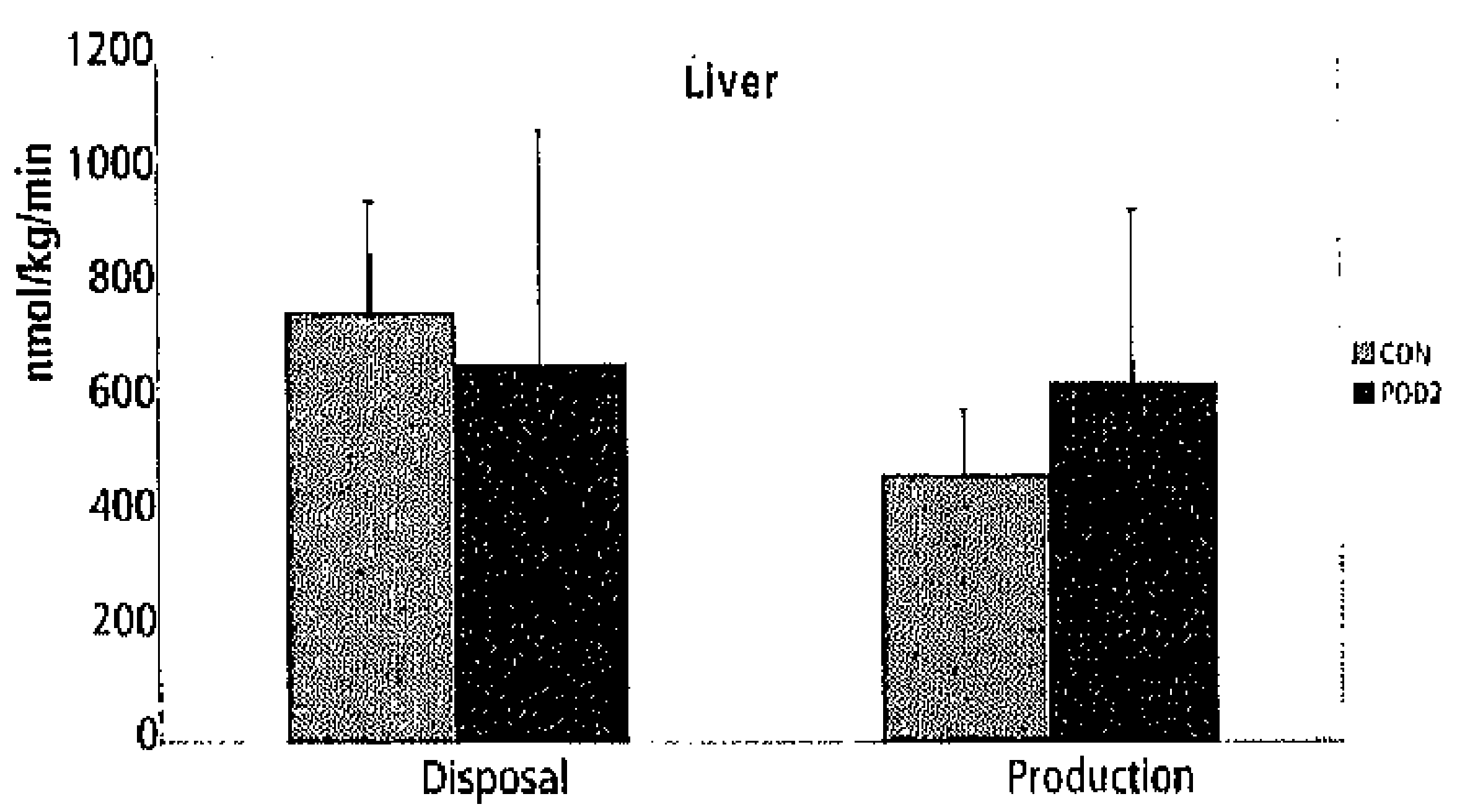

Figure 8 Phenylalanine kinetics across hindquarter and PDV and valine kinetics across the liver. CON is the control group and POD2 represents the postoperative group.

Values are expressed in $\mathrm{nmol} / \mathrm{kg} / \mathrm{min}$. 


\section{DISCUSSION}

It is assumed that 10 days after laparotomy, when clinically the pigs have recovered fully from surgery, amino acid and intermediary metabolism represent unstressed control values. Indeed, arterial glutamine concentrations and glutamine fluxes found in this study are comparable to values obtained in a similar model 2-3 weeks after surgery?. On the other hand, this model of young fast growing pigs prevents much longer recovery times because of concerns of occlusion of catheters and displacement of catheter tips in the growing pig.

The amino acid fluxes across hindquarter, PDV and liver found in this study mirror previous findings by Deutz et al. ${ }^{7}$. This can be characterized by amino acid efflux from the muscle and uptake by the splanchnic (and especially liver) tissues. Similarly, organ protein metabolism within these organs parallels findings by Bruins et al. using a porcine model of hypermetabolic sepsis ${ }^{30}$. Whole body protein turnover rates were, in contrast to what was expected, unchanged by the surgical trauma. Other studies involving healthy volunteers receiving endotoxin, or gastrointestinal cancer patients described increases in whole body protein breakdown rates 5,31 . On the other hand, whole body phenylalanine turnover remained unchanged in the immediate and delayed phase after endotoxin administration in a comparable pig model ${ }^{32}$. Possibly, the down regulating effects of fasting on whole body protein turnover may have counteracted the expected rise in whole body protein turnover after surgery. Perhaps smaller animals are more susceptible to this phenomenon compared to humans. This particularly may have played a role since food intake during the day prior to the experiment was lower directly after surgery compared to the day prior to execution of the control experiments. Since we found substantial changes in amino acid fluxes and phenylalanine production rates across the hindquarters, it is possible that due to starvation, these increases in protein breakdown may have been compensated within other organs, resulting in comparable values for whole body protein breakdown levels.

Glutamine whole body turnover figures have been more subject to discussion. On the basis of methodological issues, primarily concerning the absence of isotopic steady state, the value of the exact figures is questioned ${ }^{21}$. Overall, varying results of whole body glutamine turnover rates during disease have been reported ranging from no significant changes to increased glutamine rates of appearance $4,5,33,34$. In the current study a significant decrease in glutamine appearance rate was found. Physiological explanations include a relatively small trauma or the effects of starvation. Methodologically, the principal concern is the equilibration of the glutamine tracer with the intramuscular glutamine pool. Assuming a decrease in poolsize after trauma, and constant glutamine transport rates across the cell membrane, faster mixing with the muscle pool would occur. The result would be lower glutamine turnover rates on the whole body level. Since no muscle biopsies were taken, however, no definite explanation can be given. Data from human studies, however, support this latter mechanism ${ }^{21}$. The data regarding whole body glutamine flux should therefore be interpreted with caution.

\section{Hindquarter}

With the use of stable isotope techniques we have gained better insight in intra- and interorgan glutamine kinetics by quantification of the specific contributions of protein synthesis, degradation and glutamine synthesis and oxidation rates. 
After surgery, massive release of amino acids from the muscle occurred. Release of glutamine and alanine were quantitatively the most important. These changes can be explained in part by a net increase in muscle protein breakdown, resulting from enhanced breakdown and decreased synthesis rates, although these changes were not statistically significant. Effects of starvation affecting protein synthesis rates cannot be ruled out. The net phenylalanine production (i.e. protein breakdown) increased from 80 to $352 \mathrm{nmol} / \mathrm{kg} / \mathrm{min}$. Assuming that muscle protein contains 4.3 grams phenylalanine per $100 \mathrm{~g}$ protein 35 , this would account for 121 versus $532 \mathrm{nmol} / \mathrm{kg} / \mathrm{min}$ of glutamine production derived solely from net protein breakdown. This suggests that this source of glutamine becomes more important in the postoperative situation, increasing from $12 \%$ to $28 \%$ of the net efflux. Since glucose and lactate fluxes across the hindquarter remained similar, glucose as a precursor for the additionally alanine synthesis appears to be limited.

\section{Portal drained viscera}

The gut is very much dependent on glutamine, not only as its principal fuel, but also for DNA and RNA synthesis and possibly also for glutathione synthesis. Decreased villus height and disturbances in intestinal permeability have been ascribed to glutamine deficiencyo. In parallel with decreased arterial glutamine concentrations, uptake of glutamine by the PDV tended to decrease with $40 \%$. This finding is consistent with previous findings by our group in pigs as well as in patients 7,8 . The changes in glutamine handling by the PDV appear to be an adaptation to, rather than a cause for this decrease in glutamine supply from the plasma compartment. The gut decreases glutamine consumption and increases glutamine synthesis. Interestingly, although absolute oxidation rates decreased, relative to the total rate of disappearance they remained unchanged. The decrease can be explained by decreased glutaminase activity in the gut mucosa, an event demonstrated in starving rats?. Since no changes in glucose and lactate fluxes were detected, this would indicate that the energy consumption of the PDV decreases but that the PDV are not able to switch to glutamine as an energy source under postoperative conditions. De novo production of glutamine requires glutamine synthetase. This enzyme has been found to be active also within the small intestine ${ }^{8}$. The data provided in this study indicate that local de novo synthesis of glutamine becomes a quantitatively important source of glutamine for the gut in post surgical conditions. Possibly, effects of starvation resulting in increased glutamine synthetase activity in jejunal mucosa may also play a role? ${ }^{7}$. Furthermore, whether this glutamine production is only derived from the gut cannot be established with certainty because the small intestinal venous drainage was not sampled selectively.

The relation between arterial glutamine concentrations and glutamine uptake by the portal drained viscera indicates that the transport of glutamine into the gut is a concentration dependent process. Together with the subsequent adaptive changes occurring in glutamine metabolism within the intestine, this could provide a rationale for the provision of exogenous glutamine.

\section{Liver}

The liver is a central organ in maintaining glutamine homeostasis. In contrast to other organs, it can switch between net uptake or release of glutamine depending on the circumstances. Anatomically, the processes of glutamine production and degradation are 
compartmentalised in perivenous and pericentral hepatocytes respectively. In the control situation there was a modest release of glutamine but this changed to a significant uptake of glutamine after surgery. Thus in contrast to the gut, the liver can take up glutamine independent of the arterial concentrations. Although not statistically significant because of large variations, the trend appeared to be that more glutamine was oxidized although relative to total $\mathrm{Rd}$ of valine similar values were found. However, no significant changes in protein synthesis and degradation rates were detected. Absolute glutamine production appeared to remain stable, despite a significant relative decrease. The fate of the extra glutamine taken up can only be speculated about: since no significant changes in glucose fluxes were found gluconeogenesis does not seem to be an important goal although no direct measurements with isotopes were performed. Expected increases in protein synthesis could not be corroborated by increases in valine disposal. Oxidation rates increased suggesting a role for glutamine as a precursor for TCA intermediates. Finally, increased synthesis of oligopeptides such as glutathione may also play a significant role, since this would not affect valine kinetics.

\section{Kidney}

The kidney does not appear to play a major role in interorgan glutamine metabolism after surgical trauma. No significant changes in glutamine fluxes were found and no significant changes occurred in glutamine de novo synthesis and oxidation rates. The most striking finding was a substantial increase in glutamate uptake by the kidney. The fate of the
glutamate is unclear.

In conclusion, (inter)organ glutamine kinetics clearly react to surgical trauma. Glutamine is released in large quantities by muscle tissue, presumably related to increases in protein breakdown rates, although changes in pool size and synthesis rates cannot be discarded as contributing factors. Unfortunately, methodological problems with the use of glutamine tracers in muscle tissue did not allow us to clarify this issue. The PDV, under glutamine uptake after surgery important consumer of glutamine, appears to decrease disappearance rates and an increased dependency on liver switched from net glutamine productiondency on glutamine de novo synthesis. The oxidation rates increased and relative kidney appears to play no significant role in glutamine intes decreased. Finally, the postoperative pig model. 


\section{REFERENCES}

1. van Acker BA, von Meyenfeldt MF, van der Hulst RR, et al. Glutamine: the pivot of our nitrogen economy? JPEN J Parenter Enteral Nutr. 1990; 23:\$45-48.

2. Clowes GHA, Randall HT, Cha C-. Amino acid and energy metabolism in septic and traumatized patients. JPEN J Parenter Enteral Nutr. 1980; 4:195-205.

3. Ahlman B, Ljungqvist $O$, Persson B, Bindslev L, Wernerman J. Intestinal amino acid content in critically ill patients. JPEN J Parenter Enteral Nutr. 1995; 19:272-278.

4. Jackson NC, Carroll PV, Russell-Jones DL, Sonksen PH, Treacher DF, Umpleby AM. The metabolic consequences of critical illness: acute effects on glutamine and protein metabolism. Am J Physiol. 1999; 276:E1 63-170.

5. van Acker BAC, Hulsewé KWE, Wagenmakers AJM, Soeters PB, von Meyenfeldt MF. Glutamine appearance rate in plasma is not increased after gastrointestinal surgery in humans. J Nutr. 2000; 130:1506-1571.

o. Windmueller $\mathrm{HG}$, Spaeth AE. Uptake and metabolism of plasma glutamine by the small intestine. The journal of biological chemistry. 1974; 249:5070-5079.

7. Deutz NF:P, Reijven PL., Athanasas G, Soeters PB. Post-operative changes in hepatic, intestinal, splenic and muscle fluxes of amino acids and ammonia in pigs. Clin Sici Colch. 1992; 83:607-614.

8. van der Hulst RR, von Meyenfeldt MF, Deutz NE, Soeters PB. Glutamine extraction by the gut is reduced in depleted patients with gastrointestinal cancer. Ann Surg. 1997; 225:1 12-121.

9. Alilman B, Andersson K, Ljungqvist $O$, Persson B, Wernerman J. Elective abdominal operations alter the lree amino acid content of the human intestinal mucosa. Eur J Surg. 1995; 161:593-601.

10. Soeters IR. Glutamine: the link between depletion and diminished gut function? I Am Coll Nutr. $1090 ; 15: 195.190$.

11. Souba WW, Wilmure DW. Postoperative alteration of arteriovenous exchange of amino acids across the gastrointestinal tract. Surgery. 1983; 94:342-350.

12. Griffiths RD, Jones (C, Palmer A. Six-month outcome of critically ill patients given glutaminesupplemented parenteral nutrition. Nutrition. 1997; 13:295-302.

13. Ziegler 'T'R, Young LS, Benfell $\mathrm{K}$, et al. Clinical and metabolic efficacy of glutamine-supplemented parenteral nutrition after bone marrow transplantation. A randomized, double-blind, controlled study. Ann Intern Med. 1002; 110;821-828.

14. loudijk APJ, Rijnsburger ER, Jansen J, et al. Randomised trial of glutamine-enriched enteral nutrition on infectious morbidity in patients with multiple trauma. Lancet. 1998; 352:772-776.

15. Have ten (AAM, Bost MCF, Suyck-Wierts CAW, Bogaard van den AEJM, Deutz NEP. Simultaneous measurement of metabolic flux in portally-drained viscera, liver, spleen, kidney and hindquarter in the conscious pig. Laboratory Animals. 1996; 30:347-358.

16. Deutz NE, Bruins MJ, Soeters PB. Infusion of soy and casein protein meals affects interorgan amino acid metabolism and urea kinetics differently in pigs. J Nutr. 1998; 128:2435-2445.

17. van Fijk HM, Rooyakkers DR, Soeters PB, Deutz NE. Determination of amino acid isotope enrichment using liquid chromatography-mass spectrometry. Anal Biochem. 1999; 271:8-17.

18. Heeneman S, Deutz NE. Effects of decreased glutamine supply on gut and liver metabolism in vivo in rats. Clin Sci (Lond). 1993; 85:437-444. 
19. van Eijk HM, Rooyakkers DR, Deutz NE. Rapid routine determination of amino acids in plasma by high- performance liquid chromatography with a 2-3 microns Spherisorb ODS II column. J Chromatogr. $1093 ; 620: 143-148$.

20. van Eijk HM, van der Heilden MA, van Berlo CL, Soeters PB. Fully automated liquid-chromatographic determination of amino acids. Clin Chem. 1988; 34:2510-2513.

21. Van Acker BAC, Hulsewé KWE, Wagenmakers AJM, et al. Absence of glutamine isotopic steady state: implications for the assessment of whole-body glutamine production rate. Clin Sci (Colch). 1998; 95:330-3.40.

22. Barrett EJ, Revkin IH, Young LH, Zaret BL, Jacob R, Gelfand RA. An isotopic method for measurement of muscle protein synthesis and degradation in vivo. Biochem J. 1987; 245:223-228.

23. Moller N, Meek S, Bigelow M, Andrews J, Nair KS. The kidney is an important site for in vivo phenylalanine to tyrosine conversion in adult humans: A metabolic role of the kidney. Proc Natl Acad sci U/ SA. 2000: 07:1242-1240.

24. Tourian A, Coddard I, Puck TT. Phenylalanine hydroxylase activity in mammalian cells. J Cell Physiol. $1064: 73: 150 \cdot 170$.

25. Snimnict: Fl. Harper AE. Branched chain amino acid oxidation by isolated rat tissue preparations. Biwchim Biophys Acta. 1976; 437:477-480.

25. Levillain 0. Valine oxidation in the rat medullary thick ascending limb. Pflugers Arch. 1993; 424:398 402.

27. Thompson $\mathrm{GN}$, Pacy HJ. Merritt H, et al. Rapid measurement of whole body and forearm protein tumover using a 2H5iphenvlalanine model. Am J Physiol. 1989; 256:E631-639.

28. Wolfe RR. Radioactive and stable isotope tracers in biomedicine. 1 ed. New York: Wiley-Liss Inc.; 1992.

20. Hankard RG, Darmaun D. Sager BK. D'Amore D, Parsons WR, Haymond M. Response of glutamine metabohism to exsgenous glutamine in humans. Am J Physiol. 1995; 209:E663-E670.

30. Bruins MI, Dentz NE. Soeters PB. Aspects of organ protein, amino acid and glucose metabolism in a Foricine mndel of hypermetabolic sepsis. Clin Sci (Lond). 2003; 104:127-141.

31. Lone Y, Mathews DF. He W, Marano MA, Moldawer LL, Lowry SF. Whole body and splanchnic Eucine, ghenvialanime, and glucose kinetics during endotoxaemia in humans. Am J Physiol. 1994 $20 \mathrm{~B} \cdot \mathrm{P} 4 \mathrm{19} \cdot \mathrm{T}+25$

32. Bruir. Mi. Detz NEP. Soeters PB. Aspects of organ protein, amino acid and glucose metabolism in a porine nodel of hypermetabolic sepsis. Clin Sci /Colch/. 2003; 104:127-141.

33. Ge Da, Jahoor E. Glutamine kinetics in burn patients, Comparison with hormonally induced stress in whintures. Arch Surg. 1004: 120:1318-1323.

34. Carbonncl $F$. Messing B, Darmaun D, et al. Energy and protein metabolism in malnutrition due to

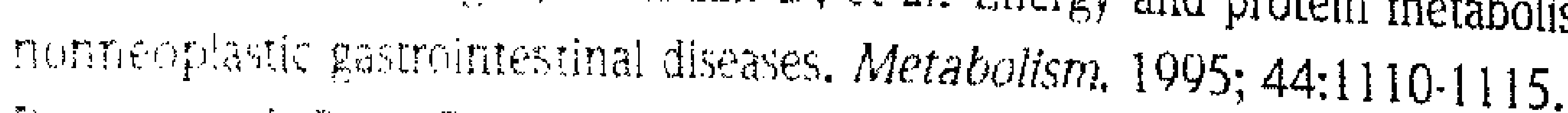

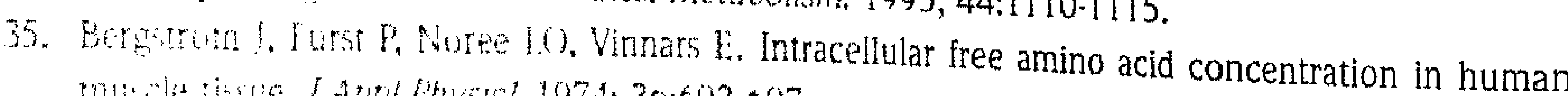

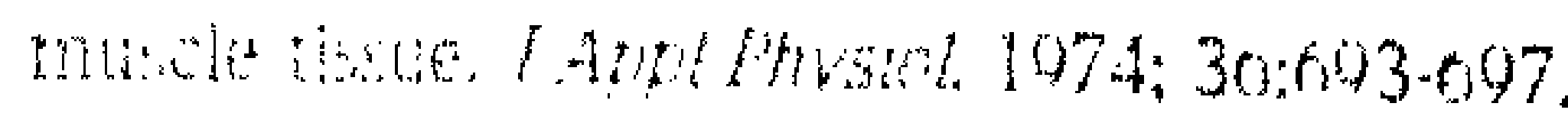

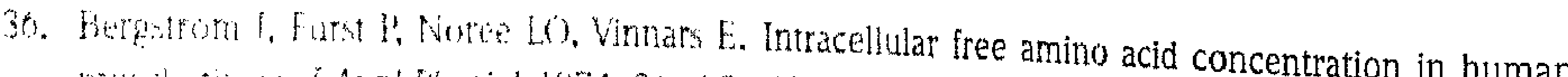

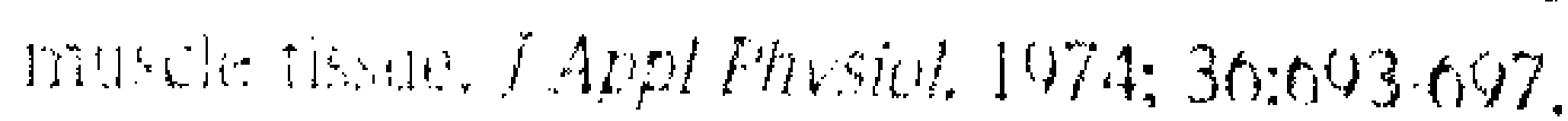

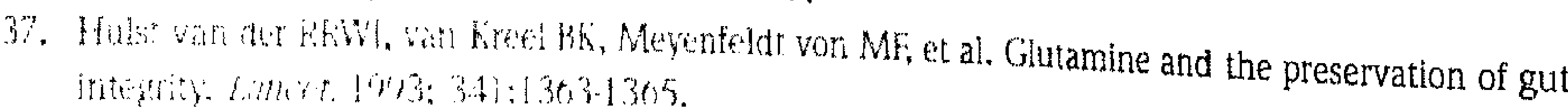

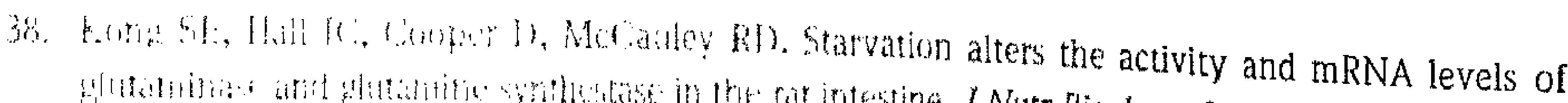

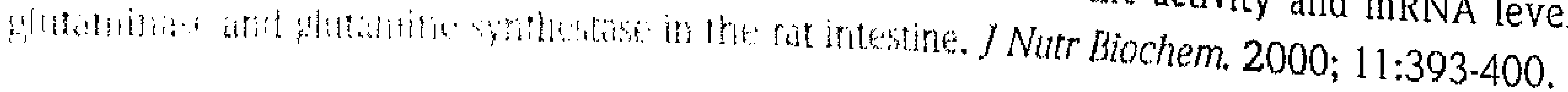


39. James LA, Lunn $P G$, Middleton $S$, Elia $M$. Distribution of glutaminase and glutamine synthetase activities in the human gastrointestinal tract. Clin Sci (Colch). 1998; 94:313-319. 

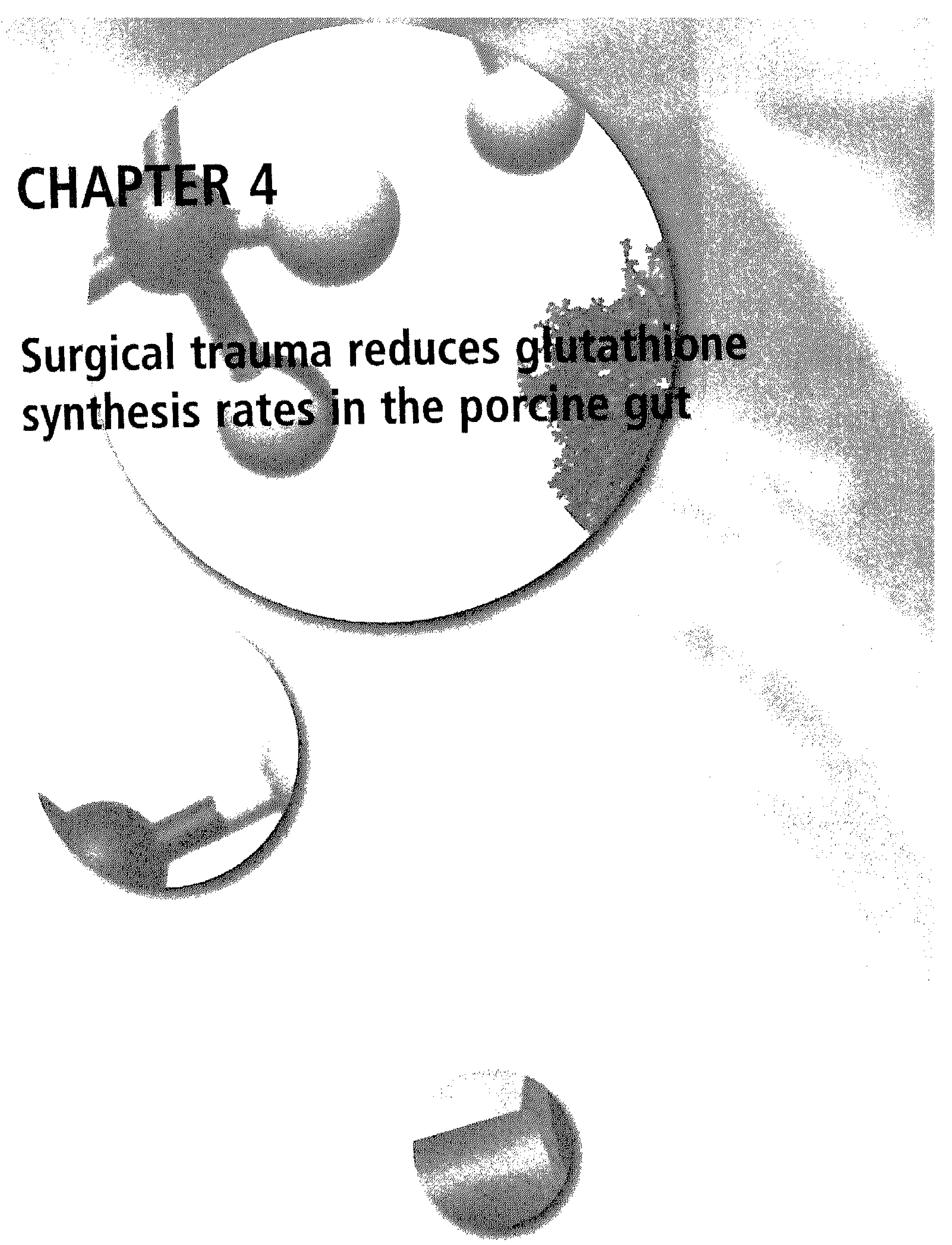

K.W.E. Hulsewé, N.E.P. Deutz', A.J. Meijer², M.F. von Meyenfeldt, P.B.Soeters

Departments of General Surgery, Academic Hospital Maastricht, 'Department of General Surgery, Maastricht University, Maastricht and 'Department of Biochemistry, Academic 
AbStRACt

Glutathione is an important tripeptide involved in the intracellular defense against free radical damage but also in signal transduction, gene expression, apoptosis and cell proliferation. The synthesis rate in the small intestine is high. Through the provision of glutamate, glutamine can affect glutamine synthesis. Since glutamine metabolism by the gut is significantly altered by surgical trauma, we studied the effects of surgery on intestinal glutathione synthesis in relation to glutamine metabolism in a porcine model using stable isotope technology.

Compared to control values we found significant decreases in gut mucosal glutamine concentrations, but no significant changes in mucosal glutathione concentrations. Absolute glutathione synthesis rates, however, decreased with $38 \%$. Although significant correlations between glutathione concentrations and glutamine and glutamate concentrations were found, these were not specific since several other amino acids showed similar correlations.

We conclude that surgical trauma decreases glutamine and glutamate but not glutathione concentrations in gut mucosa. However, absolute glutathione synthesis rates decreased. In steady state conditions this implies that loss of reduced glutathione either by oxidation or export out of the cell is decreased in the postoperative period. 


\section{INTRODUCTION}

Surgical trauma is followed by changes in protein metabolism, including an increase in protein breakdown in muscle leading to enhanced glutamine and alanine efflux from the muscle with subsequent uptake by the splanchnic organs. The splanchnic bed, and particularly the liver, has been shown to increase net protein synthesis during these so called catabolic conditions. Glutamine plays a central role in the altered amino acid interorgan exchange after trauma ${ }^{1}$. Furthermore this molecule is of great interest because of its central role in a wide array of metabolic processes such as nucleotide synthesis, acid base regulation, energy supply for rapidly dividing cells like enterocytes and immune cells, regulation of protein synthesis, interorgan nitrogen transport and gluconeogenesis ${ }^{2}$. Moreover, there is increasing evidence that glutamine is involved in the protection against the damage caused by free radicals ${ }^{3}$. Glutamine can serve as a substrate for glutathione synthesis through its deamidation to glutamate. Glutathione is the most ubiquitous free thiol in mammalian cells and is synthesized intracellularly in most cells from glutamate, cysteine and glycine. The traditional role of glutathione consists of the defense of the cell against oxidative and nitrosative stress and against free radicals. Recently, other roles in signal transduction, gene expression, apoptosis and cell proliferation have been demonstrated 4 . Within the gut all of these processes are of great importance: a large number of immune cells reside within the intestine and produce free radicals. Gene expression and signal transduction are essential functions in all tissues. The gut has a very rapid cell turnover. As a consequence apoptosis and cell proliferation are very important processes in the gut. Furthermore, the intestine is very much dependent on glutathione synthesis to maintain its concentrations. Mice treated with L-buthionine sulphoximine, an inhibitor of $\gamma$-glutamylcysteine synthetase, rapidly show decreases in the gut intracellular GSH concentration to $60 \%$ of control values within 30 minutes 5 . Glutathione, therefore, appears to be a vital compound of intestinal cells.

After surgery substantial decreases in muscle glutamine levels occur which are accompanied by decreases in muscle glutathione levels 6 . In a previous study we showed that glutamine uptake by the gut is dependent on its arterial concentration: a low arterial glutamine concentration is associated with low glutamine (fractional) extraction by the intestine ${ }^{7}$. In a porcine model we found that the postoperative decrease in arterial glutamine levels was accompanied by decreases in glutamine uptake by the gut ${ }^{8}$. However, mucosal glutamine concentrations after moderate surgical trauma and during sepsis have found to be increased in the duodenum and unaltered in the colon in humans ${ }^{9}, 10$.

Major surgery leads to increased intestinal permeability as assessed with sugar probes ${ }^{11}$. Endotoxinaemia has been found to occur during and following cardiac ${ }^{12}$ and aorta surgery 13). The reason for these changes remains unclear, but it has been suggested that under these circumstances ischemia and reperfusion of the gut lead to local damage resulting in a breakdown of the intestinal barrier. Glutathione may play an important defensive role in this process. No information is available however with regard to glutathione synthesis rates and glutathione levels in the intestine after surgical trauma. The goal of this study was to determine the effect of surgery on gut mucosal glutamine and glutathione levels as well as mucosal glutathione synthesis rates. Furthermore the relation between glutamine kinetics across the gut and mucosal glutathione synthesis was studied. 


\section{MATERIALS AND METHODS}

\section{Animals}

Female crossbred (Yorkshire x Dutch Landrace) piglets aged 10-12 weeks and weighing 18 . $22 \mathrm{~kg}$ were used for the study. They were allowed to adapt to the new circumstances for at least 1 week in galvanized bar runs ( $2 \times 3$ meter) which were equipped with isolated floors and water nipples. The temperature was held at $22.25^{\circ} \mathrm{C}$ and lights were switched on during 12 hours per day. The pigs were fed with maintenance pellets for adult sows (Landbouwbelang, Roermond, The Netherlands; $16 \%$ raw protein). They had free access to water.

The study was approved of by the Animal Ethics Committee of the Maastricht University.

\section{Surgical procedure}

The evening before surgery an enema (A.U.V., Cuyk, The Netherlands) was administered to the animals to prevent postoperative ileus. Prior to surgery, the piglets were fasted overnight. Forty-five minutes after intramuscular administration of premedication (azoperone, $8 \mathrm{mg} / \mathrm{kg}$; Stresnil ${ }^{\oplus}$, Janssen Pharmaceutica, Tilburg, The Netherlands) the piglets were brought under anesthesia with a mixture of N2O/O2 (1:2) and halothane using a mask. Anesthesia was maintained using these same volatile anesthetics. After endotracheal intubation, antibiotic prophylaxis (lincomycin $6.25 \mathrm{mg} / \mathrm{kg}$ and spectinomycin $12.5 \mathrm{mg} / \mathrm{kg}$ ) was given intravenously. Furthermore flunixine (Finadyne, Schering-Plough, Brussels, Belgium) was administered intramuscularly for postoperative analgesia. Fluid resuscitation during surgery consisted of Ringer's lactate (500-1000 ml).

The surgical procedure consisted of a laparotomy during which catheters (Tygon, Norton Co. Akron, OH, USA) were inserted into the aorta via the deep iliac circumflex artery and similarly into the caval vein for sampling of blood and infusion of isotopes respectively. To measure the portal drained viscera, 2 catheters were placed in the splenic vein (S), one of which was positioned with its tip in the portal vein $(\mathrm{P})$ at the liver hilus. After fluoroscopic control of catheter positions, all catheters were secured with sutures and the application of cyanoacrylate. Then a gastrostomy catheter was inserted and secured in place with a purse string suture. All catheters were brought out through a tunnel made through the abdominal wall. For determination of mucosal glutathione synthesis, a Bishop Koop (BK) jejunostomy was constructed ${ }^{14}$. A suitable loop of jejunum with sufficient length of the mesenterium was located and transected. After a small hole was created in the lateral part of the abdominal wall, the distal part was exteriorized and fixed to the skin. The proximal part of the bowel was anastomosed in an end to side manner to the distal bowel, approximately $5 \mathrm{~cm}$ from the abdominal wall. A Foley catheter was inserted into the stoma and inflated carefully to prevent leakage of intestinal contents. Finally the abdominal wall was closed in layers. Total operating time was 4-5 hours.

\section{Postoperative care}

After surgery, pigs were allowed to recover in a stable covered with straw. A heating lamp was provided. Each pig wore a canvass harness to protect the catheters and to allow easy handling of the animal. Stomach retention was monitored every day and drained if necessary. Catheters were checked each day for patency, flushed with heparinized saline 
and filled with a solution containing a mixture of gentamycin and chymotrypsin to prevent infections and occlusion. On the first postoperative day food was offered. After the first experiment on postoperative day 2 (POD2), the pigs were kept in the runs on straw. They were fed approximately $1 \mathrm{~kg}$ of the sow feed per day. Ten days after surgery the pigs were clinically fully recovered and they were subjected to the same experiment as on POD2. The values obtained on day 10 reflect basal conditions 8 and were used as control values.

\section{Tracers}

After baseline blood samples were taken, a primed constant infusion of the following tracers was started: para-aminohippuric acid (PAH; $25 \mathrm{mM}, \mathrm{A} 1422$, Sigma Chemical Co.) through the $\mathrm{A} 1$ and the $S$ catheter at a rate of $2 \mathrm{ml} / \mathrm{kg} / \mathrm{h}$ after a 1 hour prime ${ }^{15}$ and ${ }^{2}{ }^{13} \mathrm{C}$ glycine (prime: $50 \mu \mathrm{mol} / \mathrm{kg}$, infusion: $50 \mu \mathrm{mol} / \mathrm{kg} / \mathrm{h}$ ). Stable isotopes were purchased from Mass Trace, Woburn, USA.

\section{Experimental protocol}

After an overnight fast, the pigs were transferred to a small movable cage where the experiment was performed under unrestrained, conscious conditions. After administration of the prime, infusion of the glycine tracer was started through the venous catheter. After steady state conditions were obtained after 2 hours ${ }^{10}$, arterial blood was sampled and a mucosal biopsy was taken through the BK jejunostomy. Infusion of the glycine tracer continued for 4 more hours, after which another biopsy was taken from the gut mucosa. Simultaneously blood samples were taken.

\section{Sample processing}

Immediately after sampling, blood was distributed in heparinized tubes on ice. After thorough mixing and centrifugation, plasma was collected for further analysis. Centrifugation was performed at $4^{\circ} \mathrm{C}$ for 5 minutes at $8.500 \mathrm{~g}$ to obtain plasma. For amino acid and glutathione analysis, plasma was deproteinized by addition of $500 \mu \mathrm{L}$ plasma to $20 \mathrm{mg}$ dry sulfosalicylic acid. Native plasma was stored for enrichment analysis of the tracer. Whole blood was collected for determination of glutathione concentrations.

Mucosal biopsies were rinsed in ice cold normal saline and after removing excess water the biopsies were snap frozen in liquid nitrogen. At the end of the experiment, wet weight of the biopsies was recorded and the biopsies were added to a vial containing $2.5 \%$ sulfosalicylicacid and glass beads. The mucosa was homogenized using a Mini Beadbeater (Biospec products, Bartlesville, USA) and frozen again in liquid nitrogen. Finally all samples were stored at $-80^{\circ} \mathrm{C}$ until analysis.

\section{Biochemical analysis}

Plasma and mucosal amino acid concentrations, including GSH were measured on a fully automated High Performance Liquid Chromatography system as described before ${ }^{17}$. Glycine and glutathione enrichments were measured on a fully automated liquid chromatographymass spectrometry system (LC-MS, Thermoquest LCO, Veenendaal, The Netherlands). Isotopic enrichments are corrected for background (= pre tracer infusion) enrichments and expressed as tracer to tracee ratio's (TTR). 


\section{Calculations}

Alpha-amino nitrogen was calculated as the sum of the individual amino acids measured 18 . Branched chain amino acids were calculated as the sum of leucine, isoleucine and valine.

Glutathione synthesis rates in the intestinal mucosa were determined by measuring the rate at which the glycine tracer is incorporated into the tripeptide. After allowing isotopic steady state to be reached, a baseline mucosal biopsy was taken 2 hours after start of tracer infusion. A second biopsy was taken 4 hours later. The intracellular glycine enrichment is assumed to reflect the enrichment of the precursor pool and was calculated as the mean of the intracellular glycine enrichment at $t=2$ and $t=6$. The fractional synthetic rate (FSR) of GSH, expressed in \% per day is then calculated as:

$$
\text { (1) } \mathrm{FSR}_{\mathrm{gsh}}=\left(\mathrm{TTR}_{0}-\mathrm{TTR}_{2} / / \mathrm{TTR}_{\mathrm{gly}} / 6 / 100 \%\right.
$$

where TTR6 and TTR2 represent enrichments of glutathione at $t=6$ hours and 2 hours respectively. TTR gly is the mean intracellular glycine enrichment. Absolute glutathione

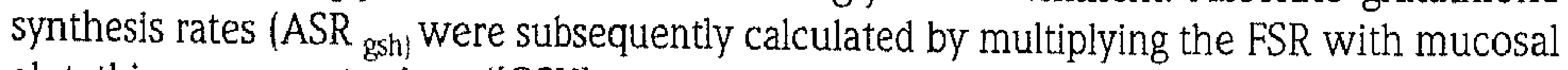
glutathione concentrations ([GSH] mucosa):

(2) ASR $_{g s h}=$ FSR $_{\text {gsh }} / \mathrm{x}[\mathrm{GSH}]_{\text {mucosa }}$

Blood flow was measured by measuring the dilution of the indicator PAH ${ }^{15}$.

(3) Flow blood = I / ([PAH]v - [PAH]a $)$

I represents the rate of infusion of PAH. [PAH]V and [PAH]a are the venous and arterial PAH concentrations respectively.

Blood flow was converted to plasma flow using the following formula:

(4) Flow plasma $=$ flow blood $-(1-$ hematocrit $)$

Substrate flux (nmol/kg bw/min) was expressed as the net balance across the specific organ and was calculated by multiplying the plasma flow with the venous [V] minus arterial [A] substrate ([AA]) concentration (5).

(5) Flux $=([\mathrm{AA}] \mathrm{v}-[\mathrm{AA} \mid \mathrm{a}) /$ plasma flow

A positive flux indicates net release whereas a negative flux reflects net uptake of the substrate across the organ.

\section{Statistics}

Results are presented as means \pm standard errors of the mean (SEM). Differences between groups were tested with the Mann Whitney $U$ test for significance. A p value of $<0.05$ was considered to be significant. Relations between glutamine fluxes across the PDV, glutamine, glutamate and glycine mucosal concentrations, mucosal glutathione concentrations, and 
glutathione synthesis rates were determined by calculating the Spearman correlation coefficient.

\section{RESULTS}

Eleven pigs underwent a laparotomy with placement of catheters and construction of a jejunostomy, and were measured on the $2^{\text {nd }}$ and the $10^{\text {th }}$ postoperative day. Results at the $2^{\text {nd }}$ day reflect the traumatized state due to the surgical procedure. Pigs are assumed to have recovered from the surgical trauma after 10 days which therefore reflects the basal control situation8.

Plasma glutamine concentrations decreased from $473 \pm 102$ to $358 \pm 77 \mu \mathrm{mol} / \mathrm{L}$ $(p=0.03)$. Glutamine concentrations in jejunal mucosa decreased from $291 \pm 23$ to $190 \pm$ $14 \mu \mathrm{mol} / \mathrm{kg}$ wet weight $(p=0.001)$ after surgery. Glutamate, alpha aminobutyric acid, phenylalanine and total amino acid concentrations all decreased significantly (table 1).

Table 1 Mucosal amino acid concentrations ( $\mu$ mol/kg wet weight)

\begin{tabular}{|c|c|c|c|c|c|c|c|}
\hline \multirow[b]{2}{*}{ Glutamate } & \multicolumn{3}{|c|}{ POD2 } & \multicolumn{3}{|l|}{ 6ONT } & \multirow[b]{2}{*}{0.012} \\
\hline & 2719 & \pm & 204 & 3623 & \pm & 229 & \\
\hline Glutathione & 1430 & & 127 & 1757 & & 156 & \\
\hline Asparagine & 98 & \pm & 14 & 102 & \pm & 12 & \\
\hline Serine & 1023 & \pm & 168 & 1233 & \pm & 218 & \\
\hline Glutamine & 190 & \pm & 14 & 291 & \pm & 23 & 0.001 \\
\hline Glycine & 69 & \pm & 7 & 69 & \pm & 6 & \\
\hline Histidine & 1600 & \pm & 157 & 1889 & \pm & 151 & \\
\hline Threonine & 284 & \pm & 25 & 341 & \pm & 28 & \\
\hline Citrulline & 184 & \pm & 24 & 204 & \pm & 34 & \\
\hline Alanine & 96 & \pm & 14 & 144 & \pm & 28 & \\
\hline Arginine & 352 & \pm & 39 & 420 & \pm & 41 & \\
\hline Taurine & 1676 & & 186 & 1911 & \pm & 110 & \\
\hline Alpha-aminobutyric acid & 118 & \pm & 17 & 41 & \pm & 6 & 0.004 \\
\hline Tyrosine & 48 & \pm & 9 & 48 & \pm & 8 & \\
\hline Valine & 257 & \pm & 35 & 194 & \pm & 16 & \\
\hline Methionine & 86 & \pm & 23 & 72 & \pm & 11 & \\
\hline Isoleucine & 414 & \pm & 45 & 334 & \pm & 26 & \\
\hline Phenilalanine & 188 & \pm & 25 & 135 & \pm & 16 & 0.028 \\
\hline Tryptophan & 423 & \pm & 79 & 278 & \pm & 20 & \\
\hline Leucine & 209 & \pm & 87 & 132 & \pm & 16 & \\
\hline Ornithine & 93 & \pm & 45 & 50 & \pm & 5 & \\
\hline Lysine & 93 & \pm & 10 & 82 & \pm & 9 & \\
\hline Sum of all amino acids & 9471 & \pm & 1227 & 13350 & \pm & 896 & 0.054 \\
\hline
\end{tabular}


Mucosal GSH concentrations did not decrease significantly $(1757 \pm 156$ versus $1430 \pm$ $127 \mu \mathrm{mol} / \mathrm{kg}^{-1}$ wet weight). Similarly, whole blood glutathione concentrations remained unchanged: control $86 \pm 11$ versus $79 \pm 16 \mu \mathrm{mol} / \mathrm{L}$ postoperatively. Glutamine uptake by the portal drained viscera decreased but did not reach significance $(p=0.07)$.

Intracellular glycine enrichments were not significantly different between 2 and 6 hours after infusion, reflecting isotopic steady state. The fractional synthesis rate in the control experiment was $190 \pm 25 \% /$ day. This did not differ significantly from the second postoperative day (129 $\pm 38 \%$ /dayl. The absolute GSH synthesis rate, however, decreased significantly from 1557 \pm 221 to $972 \pm 429 \mu \mathrm{mol} / \mathrm{kg}$ wet weight $/$ day $(p<0.05$ ) (figure 1 ). In contrast, the glycine tracer only very slowly mixed with the intracellular erythrocyte pool (data not shown). This resulted in absence of steady state conditions, precluding calculation of glutathione synthesis rates in erythrocytes.

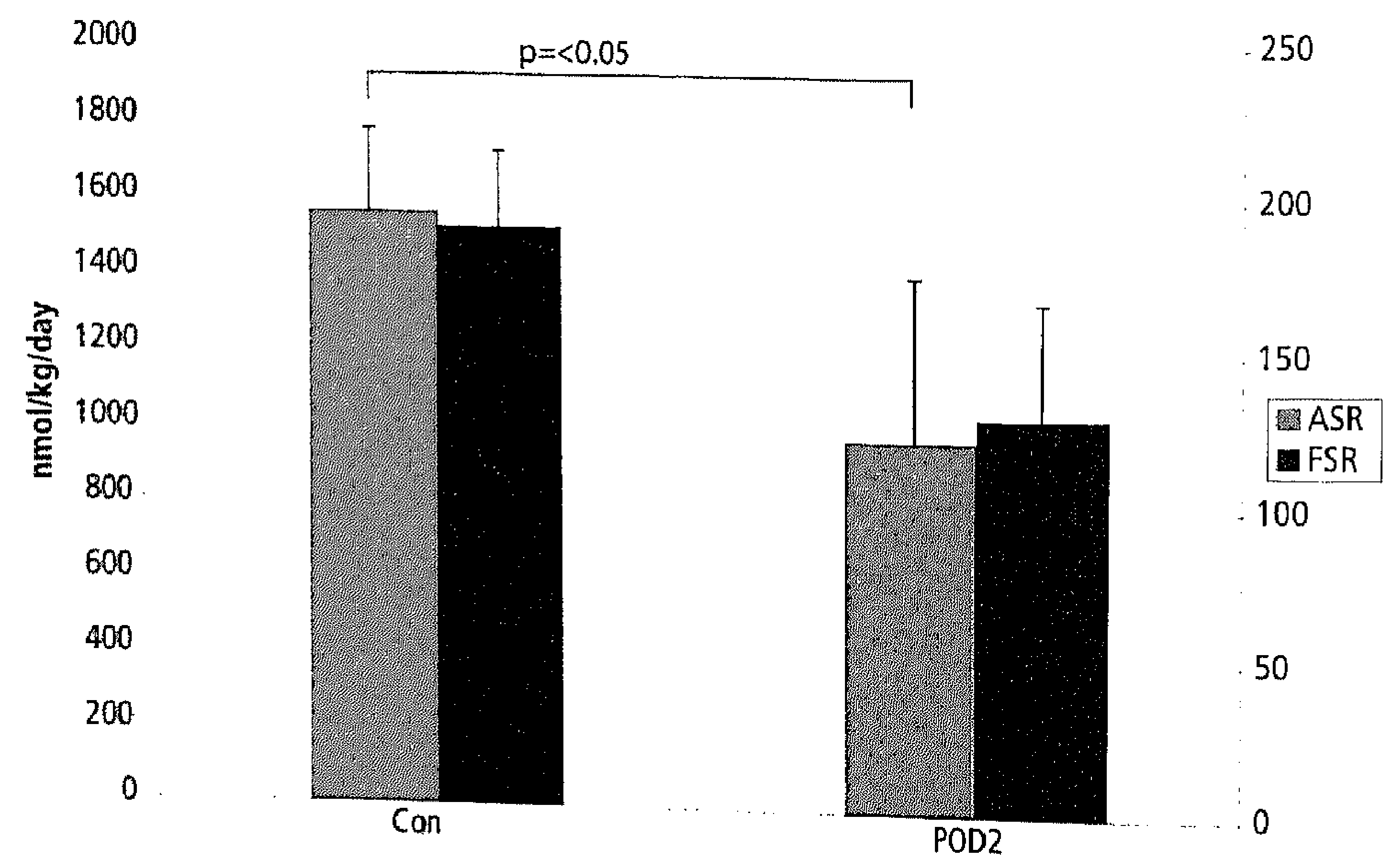

Figure 1 Effects of Surgery on gut fractional and absolute glutathione synthesis rates $\left(\mathrm{nmol} / \mathrm{kg}^{-1} /\right.$ day $\left.{ }^{l}\right)$

There were significant correlations between gut mucosal glutamate, glutamine and givcine concentrations with intestinal glutathione concentrations (fig 2 ). However, not only
these but other mucosal amino acid concentrions a these but other mucosal amino acid concentrations also correlated significantly with arginirie, taurine, tyrosine, methionine, leucine and lysine also correlated citrulline, alanine, mucosal glutathione concentrations. Clutamine concentrations correlated significantly with weakly related to absolute glutathione synthesis rates $\left(\mathrm{r}^{2}=0.12\right.$, $\mathrm{p}=0$ ificantly but only extraction by the gut was not correlated with glutathione synthesis rates. 


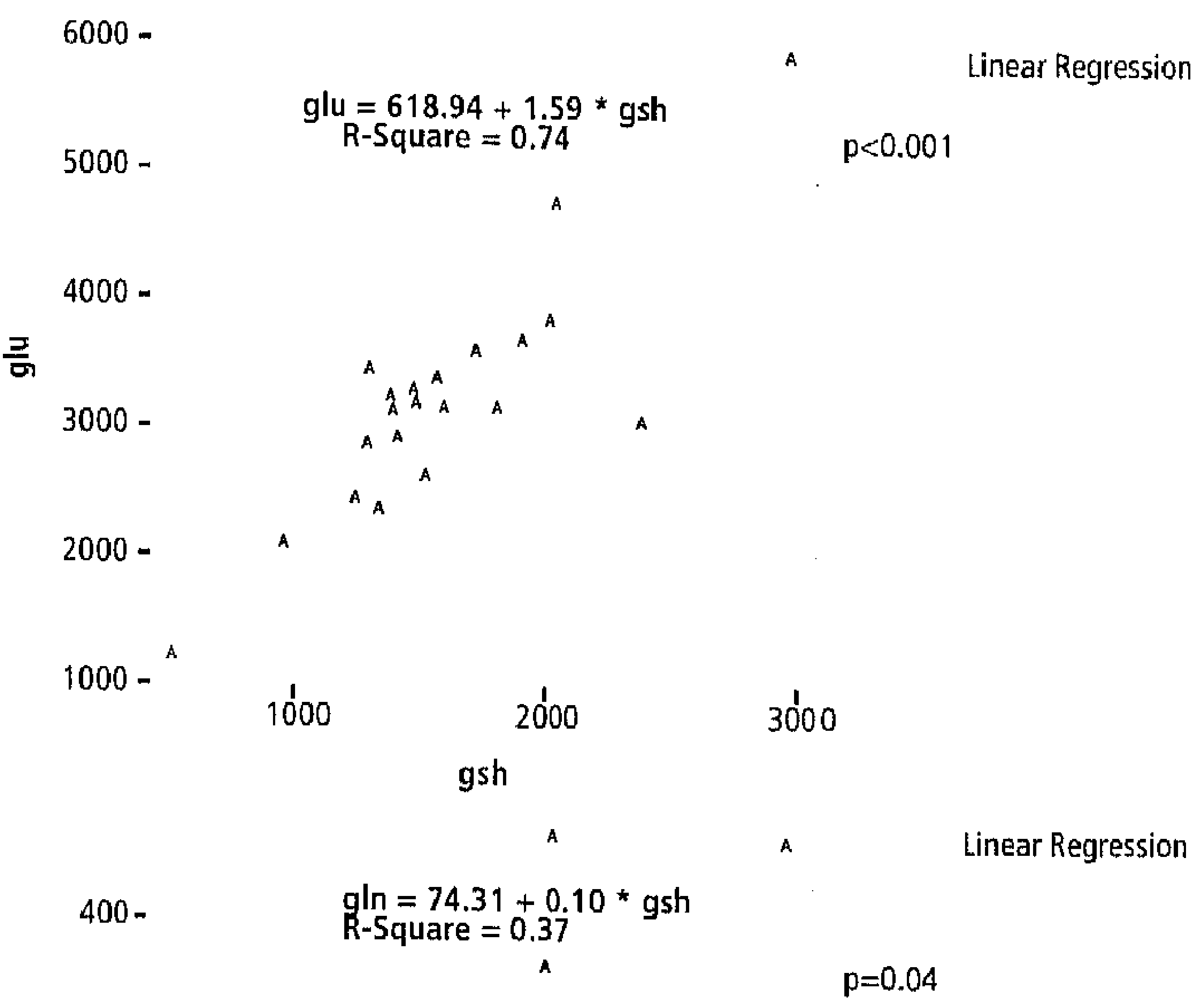

듬

$300-$
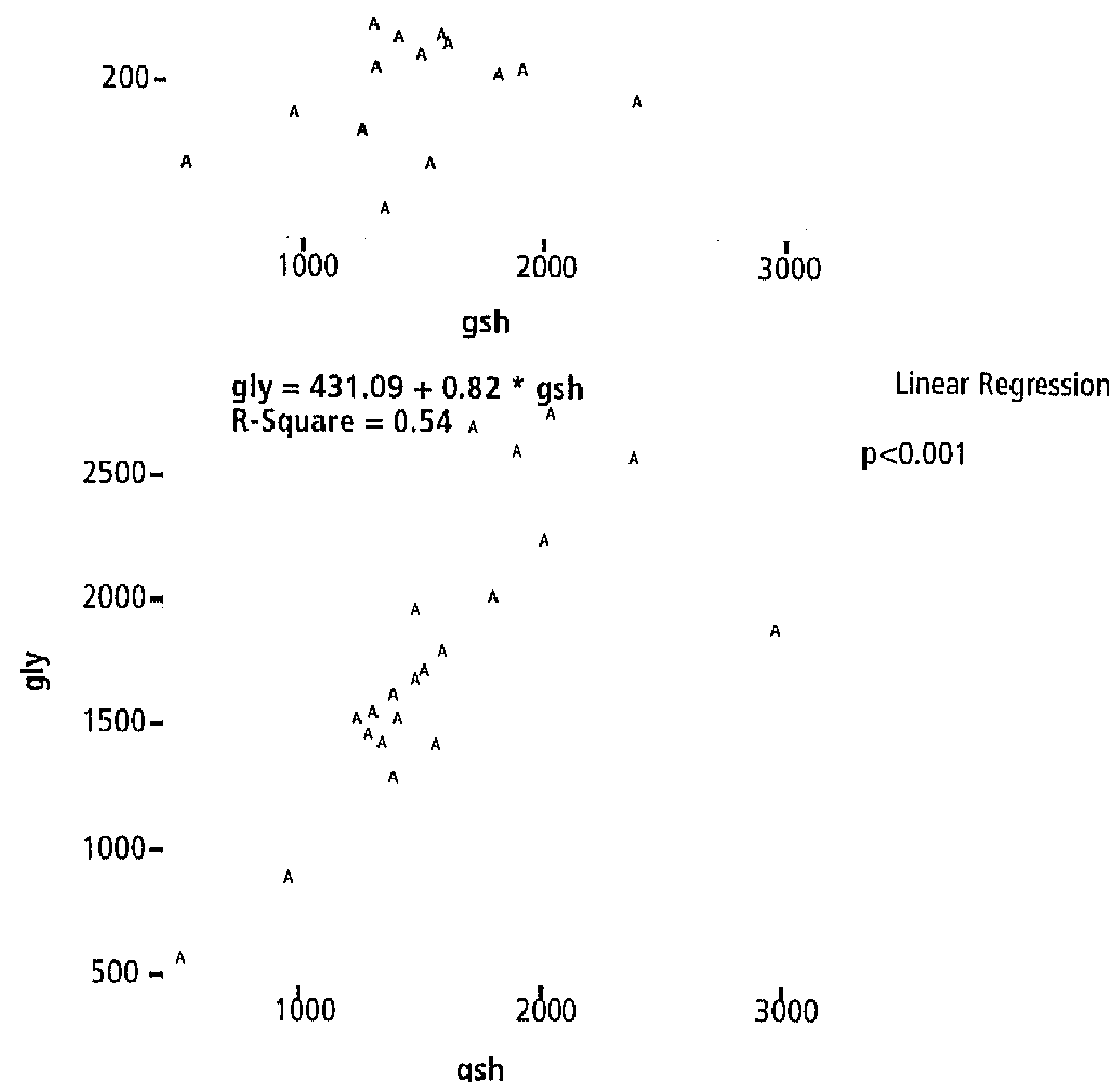

Figure 2 Correlations of mucosal glutamate (glu), glutamine (gln) and glycine (gly) concentrations with gut mucosal glutathione concentrations (gsh). Values are expressed in $\mu \mathrm{mol} / \mathrm{kg}$ wet weight. 


\section{DISCUSSION}

Since surgical trauma can adversely affect intestinal function and alter glutamine and glutamate metabolism we investigated a possible relation of glutamine with glutathione metabolism in the gut. Although glutathione was discovered already in 1888 and has elicited much research, only recently the focus has shifted to clinical aspects ${ }^{4}$. Glutathione has been shown to play a role in AIDS, cancer but also in ulcerative colitis. Moreover, several systemic inflammatory conditions have been shown to affect GSH metabolism 19-23 in different organs.

The primary hypothesis of this study was that lowered plasma glutamine concentrations, which also occur after trauma, would reduce uptake of glutamine by the bowel 7 . This in turn would lead to lower mucosal glutamine concentrations ${ }^{24}$ resulting in a decreased availability of glutamate as a precursor for GSH synthesis. This finally could impair GSH synthesis rates and possibly lower mucosal GSH levels. In muscle this mechanism has been shown to occur 25 . The present study supports the first part of the hypothesis: plasma glutamine concentrations decreased as did mucosal glutamine concentrations. Moreover absolute GSH rates decreased. However, GSH concentrations did not decrease significantly. Assuming steady state conditions, disappearance of GSH (either through oxidation or export out of the cell) must have decreased as well. Since GSH is maintained predominantly in a reduced state by GSSG reductase, GSH loss by export out of the cell is the most likely explaining mechanism ${ }^{26}$. The fact that many other amino acids showed correlations with GSH levels precludes the conclusion that the correlation between GSH and glutamine reflects a causal relationship. This probably indicates that external factors cause a nonspecific decrease in intracellular amino acid and oligopeptide concentrations after trauma. Similarly, general decreases in plasma amino acid concentrations have been demonstrated to occur after trauma and sepsis 27 . It is not likely that differences in food intake prior to the overnight fasting explain the observed effects because fasting in rats resulted in lower GSH concentrations in liver tissue but higher levels in intestinal tissues 28 . Decreased GSH concentrations in skeletal muscle after surgical trauma have been described by Luo et al. ${ }^{20}$. In skeletal muscle decreases were measured of $40 \%$ whereas we only found a modest and not significant decrease of $20 \%$ in jejunal mucosa. The fall in muscle GSH content, described by Luo et al., was related to decreased GSH synthetase levels and activities but not to glutamate or $\gamma$ glutamylcysteine concentrations. These other parameters were not measured in the current study.

Jahoor et al. have investigated GSH synthesis rates in gut mucosa in pigs21. They found GSH concentrations in gut mucosa that were 2-5 times higher compared to our results. The fractional synthesis rates were in the same order of magnitude. The reasons for the differences in concentrations are not clear, but the pigs differed in age, diet, sex and the mechanism of injury (subcutaneous terpentine versus surgical trauma). Furthermore, in the study by Jahoor et al. no values were obtained in healthy conditions. In contrast to other organs, the small intestine has a comparable FSR of GSH with the liver (170\%/day), although higher rates have been reported in rats $(465 \%)^{22}$. Erythrocytes have lower synthesis rates $(42-70 \%)$ and muscle, lung and colon have FSR of approximately $100 \% /$ day 22 .

In the current study the redox potential and specifically the GSSG/GSH ratio were not 
determined, although this ratio is an important indicator of glutathione function. High degrees of oxidative stress and similarly low GSH-reductase activities leading to decreased recycling of GSSG to GSH result in increased ratio's. Moreover, glutamine oxidation has a major impact on the redox potential of the cell through the production of NADH. The question, however, is whether decreased mucosal concentrations of glutamine indeed result in absolute decreases in glutamine oxidation. Windmueller and Spaeth estimated that in rat bowel $55-57 \%$ of the glutamine carbon skeleton was directly incorporated into $\mathrm{CO} 2$ 29,30 . At the whole body level, decreases in glutamine plasma concentrations of $26 \%$ do not result in changes in absolute glutamine oxidation rates ${ }^{31}$. No data exist with regard to the influence of changes in glutamine concentrations on oxidation rates in the bowel. On the other hand, a decreased GSH/GSSG ratio is followed by a preferential excretion of GSSG by the cell after which it is broken down. This in turn stimulates GSH synthesis rates 26 . This outcome would be in contrast to what the hypothesis of this study would predict. Thus the results of this study appear to contradict indirectly major changes in GSH/GSSG ratio occurring in the gut mucosa after surgical trauma. In muscle tissue of postoperative patients a small to no decrease in GSH/GSSG ratio has been observed 20,25 . In plasma and erythrocytes no changes have been reported in redox potential20. No data have been reported regarding gut mucosal tissue.

Although glutamate and glutamine levels rarely limit GSH synthesis, our data do not exclude this possibility. In other instances, glutamine supplementation also has been reported to increase intracellular GSH levels $25,32,33$. The underlying mechanism, however, is not necessarily directly related to the availability as a substrate. High intracellular glutamate concentrations can stimulate the System $X_{c}$ - transporter which exchanges glutamate for cystine, which can be reduced intracellularly to cysteine, thereby becoming available for GSH synthesis 26 . Furthermore, high intracellular glutamate levels can inhibit the negative feedback of GSH on $\gamma$-glutamylcysteine activities, thereby stimulating the rate limiting step in GSH synthesis ${ }^{20}$.

The gut not only obtains precursors for glutathione synthesis from the plasma but also from the luminal side. Glutathione is present in high concentrations in bile and can be taken up after its breakdown to its respective constituents by $\gamma$-glutamyl transferase, which is present in the tips of the villi, by the jejunum. The quantitative importance of this pathway, however, is not clear. For example, in rats this pathway is of minor importance $34,35$.

In conclusion, surgical trauma decreases gut glutathione synthesis rates. This is associated with decreased mucosal glutamate and glutamine concentrations, but these correlations are not specific precluding conclusions regarding causality. Future studies should focus on the potentially detrimental effects of (mild) glutathione depletion in the gut. Furthermore, possible interventions with glutamine supplementation should be further investigated in relation with glutathione synthesis.

\section{ACKNOWLEDGEMENTS}

The authors acknowledge the technical assistance of H.M. van Eijk with the analytical procedures and of G.A.M. ten Have with care of the animals. 


\section{REFERENCES}

1. Souba WW, Austgen TR. Interorgan glutamine flow following surgery and infection. JPEN J Parenter Enteral Nutr 1990; 14:90S-3S.

2. Smith RJ. Glutamine metabolism and its physiologic importance. JPEN J Parenter Enteral Nutr 1990; 14:40S-4S.

3. Mates JM, Perez-Gomez C, Nunez de Castro I, Asenjo M, Marquez J. Glutamine and its relationship with intracellular redox status, oxidative stress and cell proliferation/death. Int J Biochem Cell Biol $2002 ; 34|5|: 439-58$.

4. Sies H. Glutathione and its role in cellular functions. Free Radic Biol Med 1999; 27(9-10):910-21.

5. Martensson J, Jain A, Meister A. Glutathione is required for intestinal function. Proc Natl Acad Sci USA 1900; 87(5):1715-9.

6. Luo JL, Hammarqvist F, Cotgreave IA, Lind C, Andersson K, Wernerman J. Determination of intracellular glutathione in human skeletal muscle by reversed-phase high-performance liquid chromatography. J Chromatogr Biomed Appl 1995; 670(1):29-36.

7. van der Hulst RR, von Meyenfeldt MF, Deutz NE, Soeters PB. Glutamine extraction by the gut is reduced in depleted patients with gastrointestinal cancer. Ann Surg 1997; 225(1):112-21.

8. Deutz NEP, Reijven PL, Athanasas G, Soeters PB. Post-operative changes in hepatic, intestinal, splenic and muscle fluxes of amino acids and ammonia in pigs. Clin Sci Colch 1992; 83(5):607-14.

9. Ahlman B, Andersson K, Ljungqvist $O$, Persson B, Wernerman J. Elective abdominal operations alter the free amino acid content of the human intestinal mucosa. Eur J Surg 1995; 161(8):593-001.

10. Ahlman B, Ljungqvist $O$, Persson B, Bindslev L, Wernerman J. Intestinal amino acid content in critically ill patients. JPEN J Parenter Enteral Nutr 1995; 19(4):272.8.

11. Kanwar S, Windsor AC, Welsh F, Barclay GR, Guillou PJ, Reynolds JV. Lack of correlation between failure of gut barrier function and septic complications after major upper gastrointestinal surgery. Ann Surg 2000; 231(1):88-95.

12. Boelke E, Storck M, Buttenschoen K, Berger D, Hannekum A. Endotoxemia and mediator release during cardiac surgery. Angiology 2000; 51(9):743-9.

13. Lau LL, Halliday MI, Lee B, Hannon RJ, Gardiner KR, Soong CV. Intestinal manipulation during elective aortic aneurysm surgery leads to portal endotoxaemia and mucosal barrier dysfunction. Eur J Vasc Endovasc Surg 2000; 19(6):019-24.

14. Bishop HC, Koop CE. Management of meconium ileus: resection, Roux-en-Y anastomosis and ileostomy irrigation with pancreatic enzymes. Ann Surg 1957; 145:410.

15. Have ten GAM, Bost MCF, Suyck-Wierts CAW, Bogaard van den AEJM, Deutz NEP. Simultaneous measurement of metabolic flux in portally-drained viscera, liver, spleen, kidney and hindquarter in the conscious pig. Laboratory Animals 1996; 30:347-58.

16. Deutz NE, Bruins MJ, Soeters PB. Infusion of soy and casein protein meals affects interorgan amino acid metabolism and urea kinetics differently in pigs. J Nutr 1998; 128(12):2435-45.

17. van Eijk HM, van der Heijden MA, van Berlo CL, Soeters PB. Fully automated liquid-chromatographic determination of amino acids. Clin Chem 1988; 34(12):2510-3.

18. van Eijk HM, Rooyakkers DR, Deutz NE. Rapid routine determination of amino acids in plasma by high- performance liquid chromatography with a 2-3 microns Spherisorb ODS II column. J Chromatogr 1993; 620(1):143-8. 
19. Yu YM, Ryan CM, Fei ZW, et al. Plasma 1.5-oxoproline kinetics and whole blood glutathione synthesis rates in severely burned adult humans. Am J Physiol Endocrinol Metab 2002; 282(2):E247-58.

20. Luo IL, Hammarqvist F, Andersson K, Wernerman J. Surgical trauma decreases glutathione synthetic capacity in human skeletal muscle tissue. Am J Physiol 1998; 275 (2 Pt 1):E359-65.

21. Jahoor F, Wykes LJ, Reeds PJ, Henry JF, Del Rosario MP, Frazer ME. Protein-deficiënt pigs cannot maintain reduced glutathione homeostasis when subjected to the stress of inflammation. J Nutr 1995; $125: 1402-72$

22. Malmezat T, Breuille D, Capitan P, Mirand PP, Obled C. Glutathione turnover is increased during the acute phase of sepsis in rats. J Nutr 2000; 130(5):1239-46.

23. Sido B, Hack V, Hochlehnert A, Lipps $\mathrm{H}$, Herfarth $\mathrm{C}$, Droge W. Impairment of intestinal glutathione synthesis in patients with inflammatory bowel disease. Gut 1998; 42(4):485-92.

24. Hulst van der RRWJ, Deutz NEP, Meyenfeldt von MF, Elbers JMH, Stockbrügger RW, Soeters PB. Nutritional depletion and mucosal glutamine concentration. Clinical Nutrition 1994; 13:228-33.

25. Flaring UB, Rooyackers OE, Wernerman J, Hammarqvist F. Glutamine attenuates post-traumatic glutathione depletion in human muscle. Clin Sci (Lond) 2003; 104(3):275-82.

20. Griffith OW. Biologic and pharmacologic regulation of mammalian glutathione synthesis. Free Radic Biol Med 1990; 2719-101:922-35.

27. Vente JP, von Meyenfeldt MF, van Eijk HM, et al. Plasma-amino acid profiles in sepsis and stress. Ann Surg 1989; 20911:57-62.

28. Siegers CP, Bartels L, Riemann D. Effects of fasting and glutathione depletors on the CSH-dependent enzyme system in the gastrointestinal mucosa of the rat. Pharmacology 1989; 38(2):121-8.

20. Windmueller $\mathrm{HG}$, Spaeth $\mathrm{AE}$. Uptake and metabolism of plasma glutamine by the small intestine. The journal of biological chemistry 1974; 249(10):5070-9.

30. Windmueller $\mathrm{HG}$, Spaeth AE. Respiratory fuels and nitrogen metabolism in vivo in small intestine of rats. The journal of biological chemistry 1980; 255(1):107-12.

31. Darmaun D, Welch S, Rini A, Sager BK, Altomare A, Haymond MW. Phenylbutyrate-induced glutamine depletion in humans: effect on leucine metabolism. Am J Physiol 1998; 274(5 Pt 1):E801-7.

32. Hong RW, Heiton WS, Rounds JD, Wilmore DW. Glutamine-supplemented TPN preservers hepatic glutathione and improves survival following chemotherapy. Surgical Forum 1990; 41:9.11.

33. Harward TRS, Coe D, Souba WW, Klingman N, Seeger JM. Glutamine preserves gut glutathione levels during intestinal ischaemia/reperfusion. J Surg Res 1994; 56:351-5.

34. lantomasi T, Marraccini P, Favilli F, Vincenzini MT, Ferretti P, Tonelli F. Glutathione metabolism in Crohn's disease. Biochem Med Metab Biol 1994; 53(2):87-91.

35. Hagen TM, Jones DP. Transepithelial transport of glutathione in vascularly perfused small intestine of rat. Am J Physiol 1987; 252(5 Pt 1):G607-13. 
Surgical trauma reduces glutathione synthesis rates in the gut 


\section{CHAPTER 5}

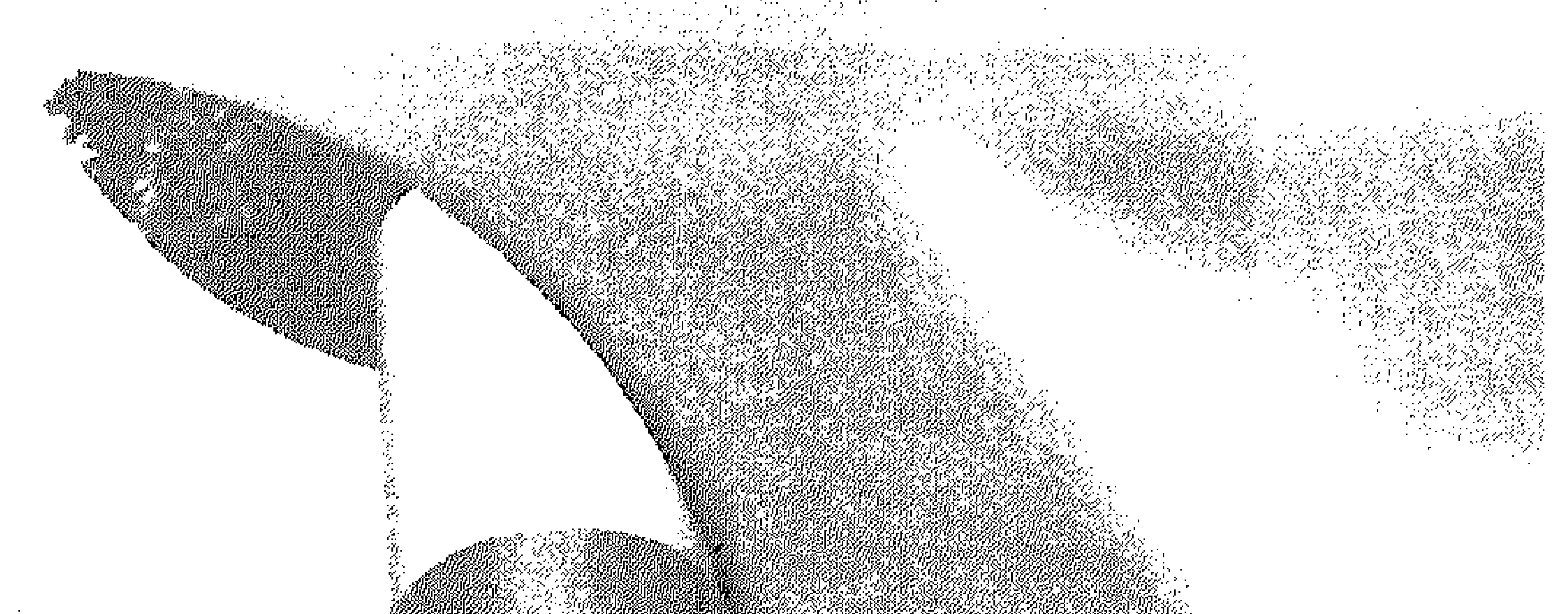

\section{effects of sepsis and pulmonary infiltrates}

Pulmonary glutamine production:

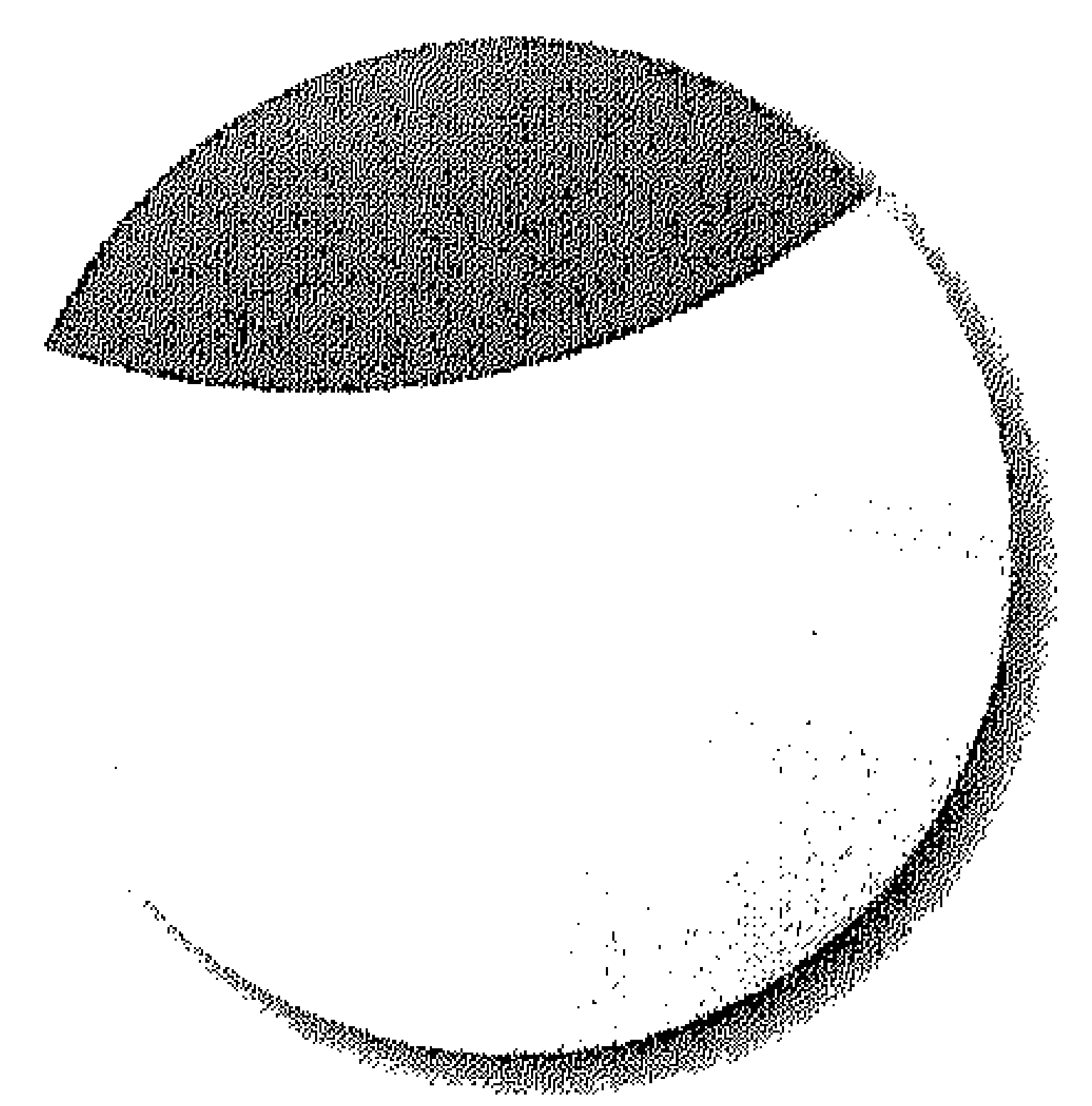

Karel WE Hulsewé MD, IRené RWJ van der Hulst MD PhD, Graham Ramsay MD PhD, ${ }^{2}$ Charles LH van Berlo MD PhD, Nicolaas EP Deutz MD PhD, Peter B Soeters MD PhD

Departments of Surgery, 1 Plastic and Reconstructive surgery, Academic Hospital Maastricht and 2Department of Surgery, Sint Maartens Gasthuis, Venlo, The Netherlands

Published in part in Intensive Care Med. 2003 Oct;29(10):1833-6. 


\section{ABSTRACT}

\section{Objective}

This study was designed to study pulmonary glutamine kinetics in a critically ill popula and to determine the effects of factors such as the presence of pulmonary inflamma. processes and the presence and severity of sepsis.

\section{Design}

Prospective clinical study

\section{Setting}

Single center; interdisciplinary intensive care unit at a university hospital

\section{Patients}

Eleven critically ill patients were compared to a population of 10 patients prior to undergc cardiac bypass surgery.

\section{Measurements and Main results}

Pulmonary arterial and arterial blood samples were taken under postabsorptive conditic Simultaneously pulmonary blood flow was determined with thermodilution. Amino : concentrations were determined in the samples. With these data AV balances and fluxe glutamine and other amino acids were calculated. Chest X-rays and calculations of severity of sepsis and multiple organ failure scores were done on the same day.

Septic patients showed significant higher glutamine efflux from the lungs compared to control population. It is estimated that at least half of this glutamine results from prol breakdown. Severity of illness had no impact on glutamine fluxes in contrast to the prese of pulmonary infiltrates on chest X-rays: in the presence of pulmonary inflammati glutamine efflux did not differ from zero.

\section{Conclusions}

The lungs play quantitatively a significant role in interorgan glutamine metabolism dur sepsis. Pulmonary infiltrates decrease the glutamine efflux from the lung in septic patie]

\section{Keywords}

glutamine, lung, sepsis, critical illness, interorgan metabolism, protein metabolism 


\section{INTRODUCTION}

The interest, that glutamine has aroused in the past decade has been primarily due to its pivotal role in diverse metabolic processes such as interorgan nitrogen transport, acid base homeostasis, DNA and RNA synthesis, regulation of protein and glutathione synthesis ${ }^{1}$. Changes in glutamine metabolism particularly occur during catabolic conditions such as after trauma or during sepsis 2.7 . Muscle, containing more than $90 \%$ of the free glutamine in the body, quickly loses most of this free glutamine under these conditions ${ }^{3}$, whereas other organs such as the immune system appear to consume increased amounts of glutamine. Glutamine supplementation in clinical practice has yielded the most promising results under severely catabolic circumstances ${ }^{9} \cdot 12$. However, the exact mechanism responsible for these clinical effects remains to be elucidated. For this purpose the roles of the different organ systems in glutamine interorgan metabolism in humans need to be clarified. Although muscle has been found to be the major provider of glutamine during stress, the contribution of the lungs under these conditions has not been convincingly defined yet. In rat experiments ample evidence has been provided that the lungs produce significant quantities of glutamine after injury or an inflammatory stimulus ${ }^{13-16}$. Studies in humans are scarce, however, and have yielded equivocal results. Two groups studied the effect of open heart surgery on pulmonary glutamine production. Herskowitz et al. showed net glutamine production rates whereas van Berlo et al. observed no significant glutamine release rates in a similar patient population ${ }^{17,18}$. Only Plumley et al. have provided data in septic patients. In their study glutamine production rates were $850 \%$ higher compared to preoperative controls. However, the inclusion criteria for these septic patients ${ }^{19}$ were solely based on physiological criteria (including a high cardiac output). Less than $50 \%$ of this population was found to have positive blood cultures, and one third of the population consisted of burn patients, who may have a different metabolic response. Lung glutamine production rates in the burn patients were $50 \%$ higher, compared to other "septic" patients, although this was statistically not significant.

Because of the uncertainty regarding the exact role of the lung in the intermediary metabolism of glutamine under catabolic conditions we undertook this study to quantify glutamine uptake and release of the human lung in septic and critically ill patients. In addition, the effects of the presence and severity of sepsis, organ failure and nutritional state were studied. Finally we wanted to define the influence of the presence of inflammation in the lungs on the production or uptake of glutamine. We hypothesized that the influx and presence of activated immune cells in the lung would increase local consumption of glutamine, thereby reducing net glutamine efflux from the lung.

\section{Patients AND MEthods}

\section{Patients}

Two patient groups were considered for entry in the study: the control group consisted of patients admitted for elective coronary artery bypass surgery. Patients were excluded when one of the following factors were present: abnormalities on a preoperative chest X-ray, 
significant recent weight loss, corticosteroid medication, diabetes mellitus, presence of malignant disease and disturbances in renal or hepatic function. The study of these patients took place prior to surgery after an overnight fast. In total 10 patients were included. Part of the data of these patients have been published before ${ }^{18}$. The second group consisted of 11 critically ill patients admitted to the general intensive care unit of our hospital. Among them there were 8 patients who were considered septic according to Bone's criteria ${ }^{20}$. These patients were studied twice during their stay in the ICU under postabsorptive conditions. Temperature, mean arterial pressure, cardiac output and ventilatory parameters such as the ventilatory mode, amount of PEEP and blood gas analysis were recorded on all study days. Of all patients daily chest X-rays were taken, which were analyzed by an independent radiologist who was not involved in the study. Severity of sepsis (SSS)scores and MOF scores were calculated on all study days ${ }^{21}$. The study was executed according to the guidelines of the local Medical Ethical Committee.

\section{Study procedure}

For determination of pulmonary glutamine fluxes arterial and pulmonary artery blood samples were drawn from an arterial catheter and a Swan-Ganz catheter respectively. The position of the central catheter was confirmed after insertion with X-ray and pressure curves. Cardiac output was measured using a thermodilution method as described by Ganz ${ }^{22}$. Pulmonary fluxes are calculated by multiplying the concentration differences of the respective amino acids between arterial and pulmonary arterial blood with cardiac output. These values were indexed to body weight. Negative fluxes indicate uptake and positive fluxes represent net substrate production by the lungs.

\section{Sample processing}

Blood samples were placed on ice immediately after collection. The sample tubes were centrifuged during 5 minutes with $3500 \mathrm{rpm}$ to obtain plasma. This was deproteinized by addition of $5 \%$ sulfosalicylic acid. After mixing these samples they were snap frozen in liquid nitrogen and stored at $-80^{\circ} \mathrm{C}$ until analysis. Amino acid analysis was performed using an automated HPLC technique as described before 23 .

\section{Statistics}

All values are expressed as mean \pm standard error of the mean (SEM).

To assess the effects of sepsis on pulmonary amino acid metabolism we compared the values of all critically ill on the first day measured and all septic patients on the days of their maximal SSS and MOF scores respectively, with the values obtained in preoperative patients. Differences between both groups were tested with the Mann-Whitney U test for significance. To determine possible effects of the severity of sepsis and body mass index (BMI), on glutamine and total amino acid flux across the lungs, data of the first study day of all critically ill patients were tested for significant correlations by calculating Spearman's correlation coefficient. The effects of pulmonary infiltrates on glutamine fluxes in critically ill patients were tested separately against zero with a one sample T-test. A p-value of $<0.05$ was considered to be statistically significant. 


\section{RESULTS}

The control group consisted of 10 patients undergoing cardiac bypass surgery with a mean age of $60.1 \pm 1.5$ years of whom 8 were men. They were all elective cases. Eleven critically ill patients were included in the study. The mean age of these critically ill patients was 62.2 \pm 3.7 years and included 6 males and 5 females. One septic patient was excluded from analysis because of the presence of upper gastrointestinal bleeding, since the blood present in the gut lumen causes significant changes in amino acid metabolism ${ }^{24}$. Data with regard to underlying disease and disease severity are summarized in table 1.

\section{Table 1 patient characteristics}

\begin{tabular}{lllll}
\hline Patient & Sex & Age & Diagnosis & Sepsis \\
\hline A & Male & 71 & Aspiration pneumonia & Yes \\
B & Female & 34 & Eclampsia & No \\
C & Female & 74 & Interstitial lung disease & No \\
D & Male & 65 & Mediastinitis & Yes \\
E & Male & 46 & Poly trauma & Yes \\
F & Female & 66 & duodenal surgery for polyp & Yes \\
G & Female & 68 & Pneumonia & No \\
H & Female & 74 & Cardiac insufficiency & Yes \\
I & Male & 64 & Sepsis eci & Yes \\
J & Male & 60 & urosepsis & Yes \\
\hline
\end{tabular}

Cardiac output, arterial glutamine levels and pulmonary glutamine fluxes were not statistically different between the preoperative patients and the group of all critically ill patients (data not shown). However, the presence of sepsis led to higher figures for cardiac output (CO) in the septic group than in the control group $(93 \pm 11 \mathrm{ml} / \mathrm{kg} / \mathrm{min}$ versus 78 $\pm 7 \mathrm{ml} / \mathrm{kg} / \mathrm{min}$ in septic patients). Arterial glutamine concentrations in septic patients (512 $\pm 59 \mu \mathrm{mol} / \mathrm{L}$ ) were not different from the control group $(519 \pm 38 \mu \mathrm{mol} / \mathrm{L})$, but arteriovenous differences of glutamine across the lungs showed release of glutamine in septic patients $(15 \pm 7 \mu \mathrm{mol})$ in comparison to net uptake in the control populations $(-45$ $\pm 28 \mu \mathrm{mol})$. This difference was borderline significant $(\mathrm{p}=0.055)$. Pulmonary glutamine flux was significantly different between both groups: septic patients produced $1412 \pm 564$ $\mathrm{nmol} / \mathrm{kg} / \mathrm{min}$ glutamine whereas preoperative patients showed an uptake of $4431 \pm 2816$ $\mathrm{nmol} / \mathrm{kg} / \mathrm{min}$. Furthermore, significant increases in pulmonary release of citrulline and total amount of amino acids were found in septic patients (table 2).

Assuming a phenylalanine and glutamine content of lung protein that do not differ from muscle or total mammalian protein ( 4.3 and 4.5 grams/100 gram protein respectively 25 ), glutamine derived from protein breakdown and consequently from de novo synthesis can be deduced from the net phenylalanine flux across the lungs. Using this calculation it is assumed that phenylalanine is not hydroxylated within the lungs ${ }^{26}$. The calculated net 
Table 2 Pulnonamy amino acid fluxes $/$ nmol $/ \mathrm{kg} / \mathrm{min} /$. Data are presented as mean \pm SEM

\begin{tabular}{|c|c|c|c|c|c|c|c|}
\hline \multirow{2}{*}{$\frac{\mathrm{AA}}{\text { Asparagine }}$} & \multicolumn{3}{|c|}{ Control } & \multicolumn{3}{|c|}{ Sepsis } & \\
\hline & 2 & \pm & 190 & 283 & \pm & 138 & \\
\hline Serine & -64 & \pm & 202 & 457 & \pm & 174 & \\
\hline Glutamine & -4431 & \pm & 2816 & 1412 & \pm & 564 & 0.014 \\
\hline Histidine & 17 & \pm & 525 & 202 & \pm & 90 & \\
\hline Ghycine & 1233 & \pm & 979 & 1086 & \pm & 321 & \\
\hline Threonine & 711 & \pm & 641 & 298 & \pm & 119 & \\
\hline Citrulline & 107 & \pm & 270 & 272 & \pm & 204 & 0.033 \\
\hline Arginine & -256 & \pm & 420 & 101 & \pm & 154 & \\
\hline Alanine & 126 & \pm & 824 & 752 & \pm & 518 & \\
\hline Taurine & 211 & \pm & 365 & -4 & \pm & 434 & \\
\hline Alpha-aminobutyric acid & -57 & \pm & 123 & 54 & \pm & 54 & \\
\hline Tyrosine & -169 & \pm & 247 & 209 & \pm & 118 & \\
\hline Valine & -948 & \pm & 1123 & 685 & \pm & 301 & \\
\hline Methionine & $\cdot 17$ & \pm & 129 & 12 & \pm & 61 & \\
\hline Bsoleucine & -408 & \pm & 438 & 116 & \pm & 65 & \\
\hline Phentilatanine & -151 & \pm & 362 & 220 & \pm & 177 & \\
\hline Tryptophan & -76 & \pm & 174 & 0 & \pm & 117 & \\
\hline Leucine & -267 & \pm & 755 & 433 & \pm & 134 & \\
\hline Ornithine & 1368 & \pm & 889 & 800 & \pm & 401 & \\
\hline Lysine & 94 & \pm & 313 & 687 & \pm & 257 & \\
\hline Branched chain amino acids & -1623 & \pm & 2283 & 1234 & \pm & 442 & \\
\hline Sum of all amino acids & 3688 & \pm & 4309 & .8325 & \pm & 2656 & 0.025 \\
\hline
\end{tabular}

protein breakdown rate amounted to 1.13 gram of protein $/ \mathrm{kg} /$ day. This results in an estimated $44 \%$ of glutamine being produced de novo. These results together with similar calculations on the basis of pulmonary leucine efflux are summarized in figure 1.

No significant correlations between the severity of sepsis (expressed as SSS and MOF scores) and body mass index with pulmonary glutamine flux were detected.

On the other hand, we found differences in pulmonary glutamine kinetics depending on the presence of inflammatory processes in the lung: in the absence of positive findings on the chest X-ray glutamine efflux from the lung was significantly different from zero as opposed to the group in whom infiltrative changes were detected (see table 3). Fifty percent of all critically ill patients demonstrated infiltrates on their chest X-ray. Similar significant differences were found for the branched chain amino acids and the sum of all amino acids. 


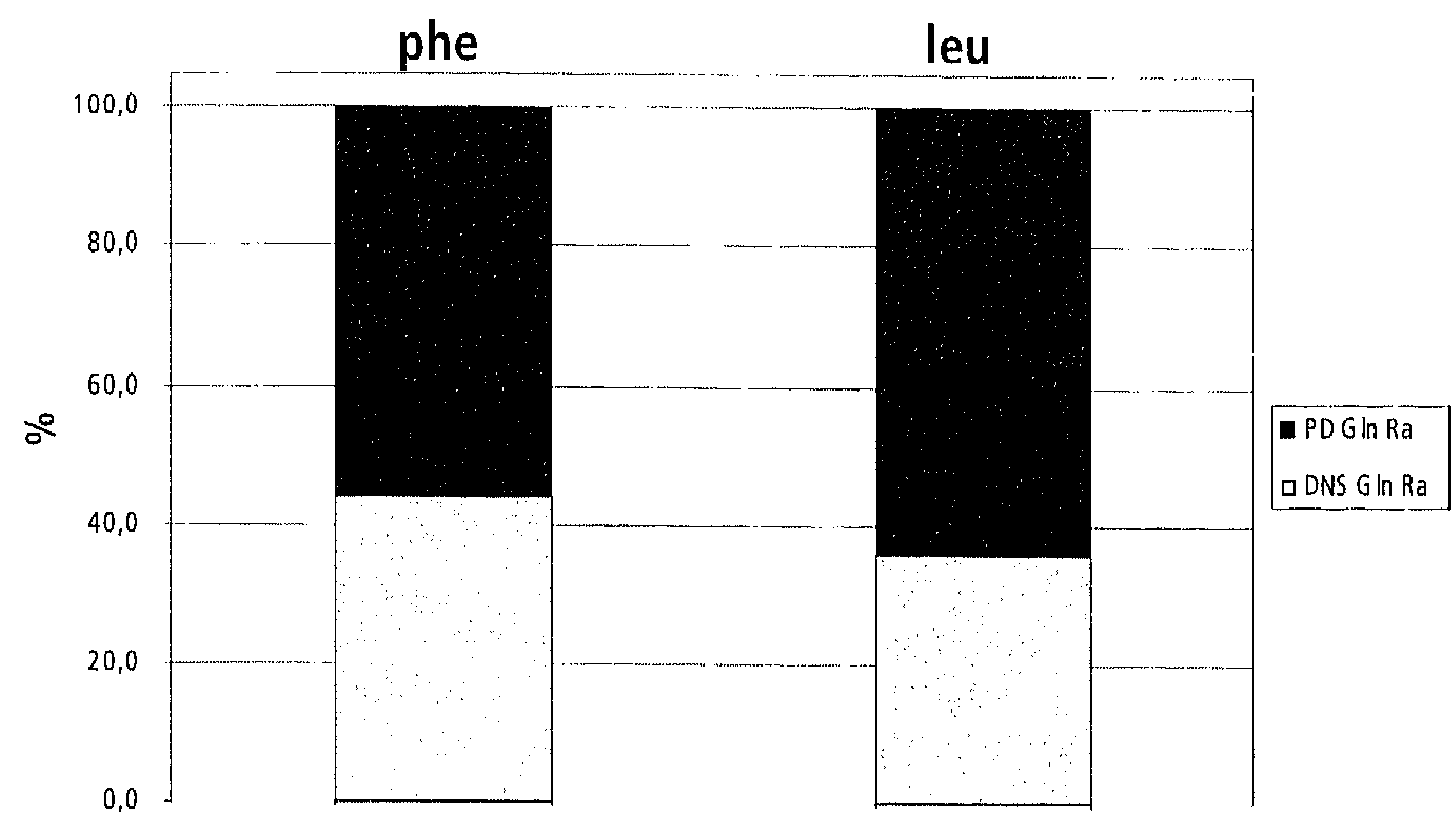

Figure 1 Lung glutamine production and proteolysis in sepsis. The bars represent the total rate of glutamine appearing from the lungs in \%. The solid parts indicate the part of glutamine derived from proteolysis (PD Gln Ra), the open parts the amount of glutamine synthesized de novo (DNS Gln Ra).

The left bar represents the data calculated with the phenylalanine flux, the right bar the data derived from calculations with the leucine flux.

Table 3 Effect of infiltrative changes on chest X-ray on lung glutamine, branched chain amino acid (BCAA) and total amino acid (SumAA) fluxes. Data are expressed as means \pm SEM. Significant differences of the fluxes from zero are represented by the $p$ values.

\begin{tabular}{lllllllll}
\hline & \multicolumn{2}{c}{ Chest X-ray +} & \multicolumn{2}{c}{ p vs 0 } & \multicolumn{2}{c}{ Chest X-ray - } & p vs 0 \\
\hline Glutamine flux & -280 & \pm & 538 & NS & -1925 & \pm & 856 & 0.04 \\
BCAA flux & -357 & \pm & 413 & NS & -1399 & \pm & 528 & 0.02 \\
SumAA flux & 8197 & \pm & 12607 & NS & -9470 & \pm & 3420 & 0.02 \\
\hline
\end{tabular}




\section{DISCUSSION}

This study adds further support to the hypothesis that the lungs play an important role in glutamine homeostasis during sepsis.

The amount of glutamine released by the lungs in septic patients in our study was 1.41 $\mu \mathrm{mol} / \mathrm{kg} / \mathrm{min}$. This is a factor 4.5 lower compared to previously published data in septic patients ${ }^{19}$ and in patients following cardiac surgery ${ }^{17}$. There are a few possible explanations to account for these differences. Whereas the previous studies included only surgical patients, we included critically ill patients from a general ICU. The large (although not significant) difference in pulmonary glutamine efflux in burn patients compared to surgical septic patients as found by Plumley and coworkers suggest that the underlying disease may influence glutamine production rates.

Another more striking difference with the previously mentioned papers is the fact that patients with pulmonary infiltrates on their chest X-ray were included in our study. Indeed, we found that the presence of signs of inflammation in the lungs leads to diminished pulmonary glutamine efflux. It is possible that the decrease in glutamine efflux is the result of an increased use of glutamine by cells involved in the inflammatory response within the lung or a decrease in glutamine synthesizing capacity by the lung. Although this study cannot provide the answer, associated decreases in branched chain amino efflux from the lungs suggest that it is an increased use of amino acids within the lungs rather than a specific decrease in glutamine synthesis capacity which is responsible for the differences found.

In contrast to muscle, lung tissue does not contain a large pool of free glutamine. Instead, under catabolic conditions transcription and translation of glutamine synthetase is significantly increased leading to enhanced glutamine de novo synthesis rates2,14,16. Another route for glutamine production is through protein degradation. Although we did not infuse isotopically labeled amino acids in this study, we can make an estimation of pulmonary protein degradation by assuming that the net release of essential amino acids such as phenylalanine and leucine is the result of protein degradation. The data thus obtained indicate that during sepsis considerable amounts of protein are broken down in the lungs. To our knowledge no comparable calculations have been published for lung glutamine metabolism before. Previously we made similar calculations at the whole body level in patients receiving total parenteral nutrition 27 . In this study approximately $56 \%$ of the rate of glutamine appearance was explained by protein degradation leaving the major part to be derived from de novo synthesis. The present data, therefore, show that a relatively large part of the glutamine produced by the lungs is derived from proteolysis. This could indicate a high turnover rate of protein in pulmonary tissues. In addition, possible transamination of leucine and hydroxylation of phenylalanine are not taken into account. Therefore, the figures represent a lower bound estimate of true protein breakdown rates. In order to determine the relative importance of the lungs in whole body glutamine metabolism we can compare the obtained data with published figures of muscle glutamine production and whole body glutamine fluxes. The data from our study estimates the pulmonary glutamine production during sepsis of a $70 \mathrm{~kg}$ individual to be 21 grams of glutamine per day. Only 2 groups specifically studied arteriovenous balances across limbs in septic patients: Clowes et al. found an increase of glutamine production in sepsis: 14 versus 6 grams per day in 
healthy controls 28 . Fong et al. found no significant differences (24 versus 21 grams per day ${ }^{29}$. Compared to muscle glutamine production, the lungs appear to be at least as important to furnish glutamine. Whole body glutamine fluxes in healthy volunteers estimate that 69-96 grams of glutamine is turned over daily. Under diverse catabolic conditions such as surgery, sepsis and burns glutamine fluxes show a turnover of 59-105 grams per day. This results in a substantial contribution of at least $20-30 \%$ of whole body Ra by the lungs, assuming that glutamine is not taken up in the pulmonary bed. Otherwise, the contribution of the lungs to whole body glutamine turnover is even higher.

We therefore must conclude that the lungs contribute significantly to interorgan glutamine metabolism during sepsis.

In conclusion, this study confirms that the lungs are quantitatively an important source of glutamine during sepsis. A relative large part of this glutamine production can be attributed to protein breakdown. The presence of ARDS or pneumonia in this critically ill population decreased net glutamine efflux from the lungs. Whether this is the result of an increased local glutamine consumption or a decreased capacity of pulmonary glutamine synthesis remains to be determined. 


\section{REFERENCES}

1 BA van Acker, von Meyenfeldt MF, van der Hulst RR, Hulsewe KW, Wagenmakers AJ, Deutz NE, de Blaauw I, Dejong CH, van Kreel BK,Soeters PB (1999) Glutamine: the pivot of our nitrogen economy? JPEN J Parenter Enteral Nutr 23:\$45-8

2 MS Ardawi (1990) Glutamine-synthesizing activity in lungs of fed, starved, acidotic, diabetic, injured and septic rats. Biochem J 270:829-32.

3 DC Gore,Jahoor F (1994) Glutamine kinetics in burn patients. Comparison with hormonally induced stress in volunteers. Arch Surg. 129:1318-1323

4 NEP Deutz, Reijven PL., Athanasas G, Soeters PB (1992) Post-operative changes in hepatic, intestinal, splenic and muscle fluxes of amino acids and ammonia in pigs. Clin Sci Colch. 83:607-614

5 N Jackson, Carroll P, Sönksen P, Treacher D, Russell-Jones D,Umbleby M (1996) The effect of critical illness on glutamine metabolism. 4th international symposium on amino acid and protein metabolism in health and disease: nutritional implications 50

6 JM Lacey,Wilmore DW (1990) Is glutamine a conditionally essential amino acid? Nutr Rev. 48:297-309

7 WW Souba,Austgen TR (1990) Interorgan glutamine flow following surgery and infection. JPEN J Parenter Enteral Nutr. 14:905-93S

8 F Hammarqvist, Luo JL, Cotgreave IA, Andersson K, Wernerman J (1997) Skeletal muscle glutathione is depleted in critically ill patients. Crit Care Med 25:78-84

9 TR Ziegler, Young LS, Benfell K, Scheltinga M, Hortos K, Bye R, Morrow FD, Jacobs DO, Smith RJ, Antin $\mathrm{JH}$,al e (1992) Clinical and metabolic efficacy of glutamine-supplemented parenteral nutrition after bone marrow transplantation. A randomized, double-blind, controlled study. Ann Intern Med. $116: 821 \cdot 828$

10 JM Lacey, C'rouch JB, Benfell K, Ringer SA, Wilmore CK, Maguire D, Wilmore DW (1990) The effects of glutamine-supplemented parenteral nutrition in premature infants. JPEN J Parenter Enteral Nutr $20: 74 \cdot 80$

11 RD Griffiths, Jones C,Palmer A (1997) Six-month outcome of critically ill patients given glutaminesupplemented parenteral nutrition. Nutrition 13:295-302

12 P Stehle, Zander J, Mertes N, Albers S, Puchstein C, Lawin P,Furst P (1989) Effect of parenteral glutamine peptide supplements on muscle glutamine loss and nitrogen balance after major surgery [see comments|. Lancet 1:231.233

13 WW Souba, Plumley DA, Salloum RM,Copeland EM (1990) Effects of glucocorticoids on lung glutamine and alanine metabolism. Surgery 108:213-8; discussion 218-9.

14 MS Ardawi (1991) Glutamine metabolism in the lungs of glucocorticoid-treated rats. Clin Sci /Colch) $81: 37.42$.

15 Bl Labow, Abcouwer SF, Lin CM,Souba WW (1998) Glutamine synthetase expression in rat lung is regulated by protein stability. Am J Physiol 275:L877-80.

16 G Lukaszewicz, Abcouwer SF, Labow BI,Souba WW (1997) Glutamine synthetase gene expression in the lungs of endotoxin-treated and adrenalectomized rats. Am J Physiol 273:L1 182-90.

17 K Herskowitz, Plumley DA, Martin TD, Hautamaki RD, Copeland EM,Souba WW (1991) Lung glutamine flux following open heart surgery. J Surg Res 51:82-6.

18 CLH Berlo van, Hulst van der RRWJ, Massen JG, Dejong CHC, Meijerink J, Deutz NEP, Meyenfeldt von MF,Soeters PB (1995) Lung glutamine metabolism: effects of starvation, parenteral and enteral nutrition. A study in man. Clinical nutrition 
10 DA Plumley, Souba WW, Hautamaki RD, Martin TD, Flynn TC, Rout WR, Copeland EM (1990) Accelerated lung amino acid release in hyperdynamic septic surgical patients. Arch Surg 125:57-61.

20 RC: Bone, Balk RA, Cerra FB. Dellinger RP. Fein AM, Knaus WA, Schein RM,Sibbald WJ (1992) Definitions for sepsis and organ failure and guidelines for the use of innovative therapies in sepsis. The ACCP.SCCM Consensus Conference Committee. American College of Chest Physicians/Society of Critical Care Medicine. Chest 101:1044-55.

21 JC Marshall, Conk DJ, Christou NV, Bernard GR, Sprung CL,Sibbald WJ (1995) Multiple organ dysfunction score: a reliable descriptor of a complex clinical outcome. Crit Care Med 23:1638-52.

22 W Ganz, Donoso R, Marcus HS, Forrester JS,Swan HJ (1971) A new technique for measurement of cardiac output by thermodilution in man. Am J Cardiol 27:392-6.

23 HM van Eijk, Rooyakkers DR, Deutz NE (1993) Rapid routine determination of amino acids in plasma by high- performance liquid chromatography with a 2-3 microns Spherisorb ODS II column. $J$ Chromatogro20:143.8

24 CH Dejong, Meijerink WJ, van Berlo CL, Deutz NE,Soeters PB (1996) Decreased plasma isoleucine concentrations after upper gastrointestinal haemorrhage in humans. Gut 39:13-7.

25 KS Kuhn, Schuhmann K, Stehle P, Darmaun D,Furst P (1999) Determination of glutamine in muscle protein facilitates accurate assessment of proteolysis and de novo synthesis-derived endogenous glutamine production. Am J Clin Nutr 70:484-9

20 A Tourian, Goddard J,Puck TT (1969) Phenylalanine hydroxylase activity in mammalian cells. J Cell Physiol 73:159-170

27 BA van Acker, Hulsewe KW, Wagenmakers AJ, von Meyenfeldt MF,Soeters PB (2000) Response of glutamine metabolism to glutamine-supplemented parenteral nutrition. Am J Clin Nutr 72:790-5

28 GHA Clowes, Randall HT,Cha C-J (1980) Amino acid and energy metabolism in septic and traumatized patients. Journal of parenteral and enteral nutrition 4:195-205

29 YM Fong, Tracey KJ, Hesse DG, Albert JD, Barie PS,Lowry SF (1990) Influence of enterectomy on peripheral tissue glutamine efflux in critically ill patients. Surgery 107:321-6. 


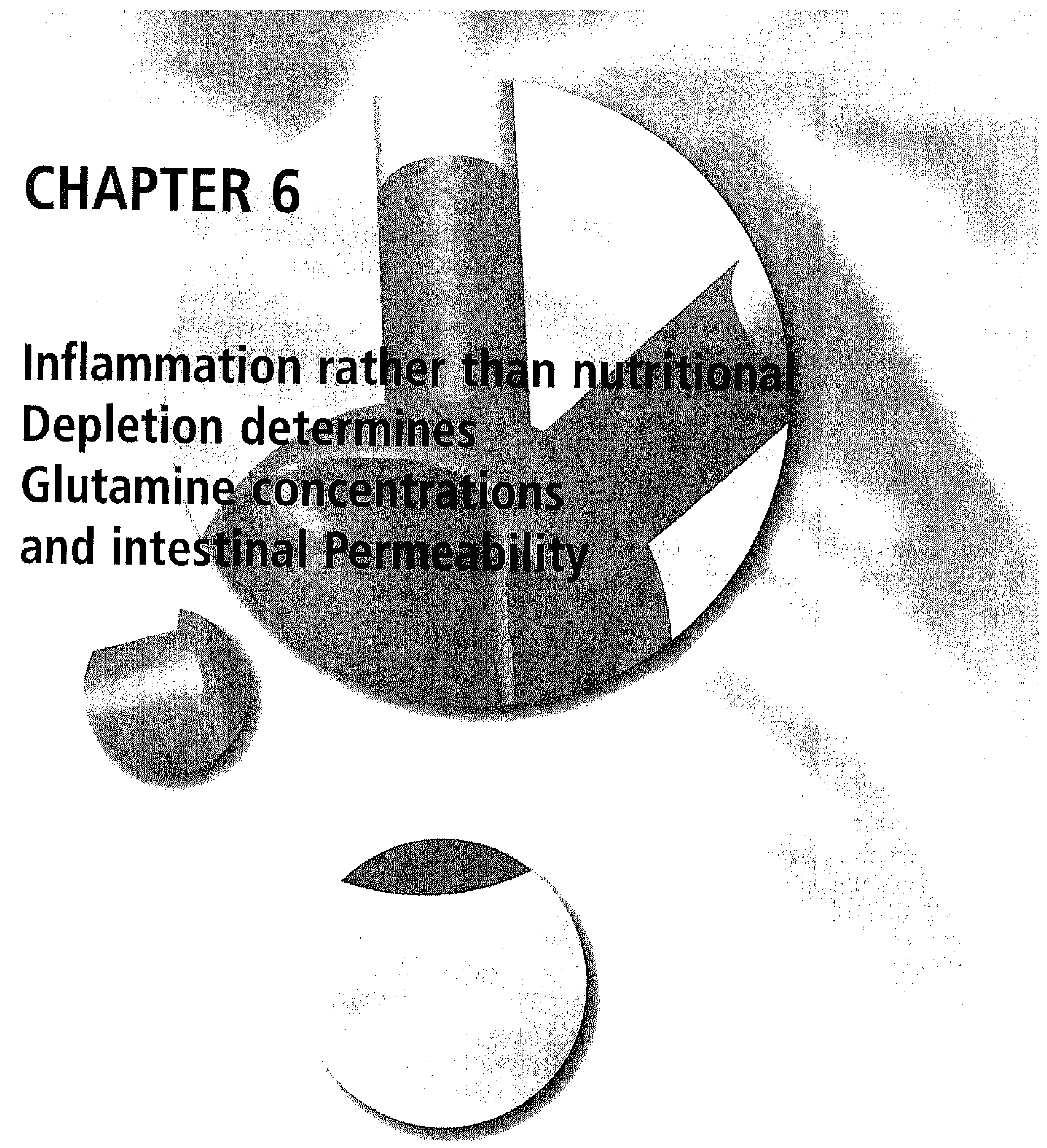

Karel WE Hulsewé, Rene WWJ van der Hulst' ${ }^{1}$, Bernadette AC van Acker, Maarten F von Meyenfeldt, Peter B Soeters

Department of General surgery, 'Plastic and Reconstructive Surgery, Academic Hospital Maastricht, The Netherlands

Published in Clin Nutr. 2004 Oct;23(5):1209-16 


\section{ABSTRACT}

\section{Aim}

Nutritional depletion has been correlated with low plasma and mucosal glutamine concentrations and with increased intestinal permeability. Since nutritional depletion often is associated with (chronic) inflammatory stress, this study was designed to establish the influence of depletion and inflammation on glutamine concentrations and gut barrier function.

\section{Methods}

Anthropometric parameters were calculated from 26 patients who required artificial nutrition. Glutamine concentrations in plasma and gut mucosa, gut permeability and mucosal morphology were assessed. For determination of the degree of inflammation erythrocyte sedimentation rates and (pre)albumin concentrations were measured. On the basis of these parameters patients were divided into two groups having significant inflammatory stress or not. Similarly a depleted and a non-depleted group was formed based on percentage ideal body weight, fat-free mass index (FFMI) and percentage weight loss. Glutamine concentrations, gut permeability and villus morphology were compared between the groups.

\section{Results}

The presence of inflammatory activity had significant negative effects on glutamine concentrations in contrast to the presence or absence of nutritional depletion. Similarly, intestinal permeability increased during active inflammation but not in depleted patients. FFMI but not inflammation was related to villus height.

\section{Conclusions}

The presence of inflammation significantly affects glutamine concentrations and gut permeability, in contrast to the presence of depletion of body cell mass per se. On the other hand, villus morphology is not influenced by changes in systemic inflammatory activity whereas nutritional status possibly does affect villus height. 


\section{INTRODUCTION}

Even though glutamine has been found to play a pivotal role in a wide variety of metabolic processes, prospective randomized controlled trials studying its supplementation have yielded conflicting results 1.5 . We found positive effects of glutamine supplementation to total parenteral nutrition (TPN) on gut barrier function 3 . Elsewhere in this journal we present the results of parenteral glutamine supplementation to a nutritionally depleted patient population ${ }^{6}$. This population had been selected on the basis of our observations that nutritional depletion correlates to low plasma and low mucosal glutamine concentrations 7,8 . To our surprise and in contrast to previous studies no effects of glutamine supplementation were found on plasma and mucosal glutamine concentrations, gut permeability and villus height. A major difference with a previously performed study of glutamine supplementation ${ }^{3}$ consisted of the characteristics of the patients included. Whereas this latter study had included mostly patients with exacerbation of inflammatory bowel disease (IBD), the recent study included more (colonic) cancer patients. Furthermore, the patients with IBD who were included in the recent study had no acute exacerbation of their disease but rather a chronic small bowel stenosis, leading to obstruction and loss of body mass. This was also reflected by differences in markers of inflammation: in the study by van der Hulst et al. ${ }^{3}$ the depleted group showed more signs of inflammatory activity than the group included in the study by Hulsewe et al. ${ }^{\circ}$. Albumin and total protein concentrations were lower whereas the leucocyte count and thrombocyte count were slightly higher (data not shown).

Unchanged glutamine concentrations after supplementation in humans has been described before 4 . Furthermore, lack of effects of glutamine on gut permeability has also been reported by others9. The reason for these conflicting results has not been clarified yet. As long as the mechanisms underlying these differences in outcome remain obscure, it will remain difficult to predict the efficacy of glutamine supplementation in the diverse clinical situations.

Nutritional depletion is frequently associated with increased inflammatory activity. Especially in patient populations in hospitals, depletion is rarely the result of pure starvation but rather a combination of decreases in oral intake due to the disease or treatment and the consequences of long standing inflammation resulting in disturbed intermediary metabolism and hypermetabolism ${ }^{10}$.

Furthermore, major changes in glutamine metabolism take place during inflammatory stress and glutamine supplementation has been most successful under these circumstances. On the other hand, the absence of systemic inflammation may result in failure to achieve clinical effects by glutamine supplementation. The goal of the present study was to determine the influence of the presence of systemic inflammation on glutamine concentrations, gut permeability and mucosal morphology.

\section{PATIENTS AND METHODS}

For this study patients were included prior to receiving preoperative total parenteral nutrition. All patients gave their informed consent. The study was approved by the Medical 
Ethical Committee of the Academic Hospital Maastricht. The data were collected in the postabsorptive state prior to administration of TPN.

\section{Patients}

Patients were eligible for inclusion in the study if they required total parenteral nutrition for at least 7 days. Furthermore, patients had to have an intact proximal gut. Patients were excluded if there was renal or hepatic insufficiency, if they had diabetes mellitus or if they were septic. After obtaining informed consent, blood was sampled and duodenal biopsies were taken.

\section{Study design}

After inclusion in the study body composition was assessed by calculation of the percentage ideal body weight (PIW) from body length, body weight and wrist circumference using the Metropolitan Life Insurance tables ${ }^{11}$. Furthermore, the fat free mass index (FFMI) was calculated by determining the percentage fat-free mass of the ideal fat-free mass, defined as $88 \%$ of ideal body weight for men and $78 \%$ of ideal body weight for women. The fat free mass was calculated from bio-impedance measurements ${ }^{12}$. The percentage of weight lost [PWL) in the 6 months preceding hospital admission was recorded. Blood samples were taken for determination of plasma amino acid concentrations and inflammatory parameters such as the erythrocyte sedimentation rate (ESR), leucocytes and (pre)albumin. Furthermore, a gastroduodenoscopy was performed during which mucosal biopsies were taken from the descending part of the duodenum. Then, after voiding, a sugar probe solution containing $10 \mathrm{~g}$ of lactulose and $0.5 \mathrm{~g}$ mannitol dissolved in $65 \mathrm{ml}$ water was infused into the duodenum distal to the site of the biopsies. Urine was collected during the next 5 hours for determination of the excretion of these sugar probes.

\section{Analyses}

- Plasma and mucosal amino acid concentrations

Mucosal biopsies were rinsed in ice cold normal saline and after removing excess water with filter paper the biopsies were snap frozen in liquid nitrogen. After recording wet weight the biopsies were homogenized with glass beads in $2.5 \%$ sulfosalicylic acid and stored at $-80^{\circ} \mathrm{C}$ until analysis. Amino acid concentrations were determined in arterial plasma and in the supernatant of the biopsies using a fully automated high pressure liquid chromatography method as described previously 13 .

- Mucosal morphology

After fixation mucosal biopsies were embedded in paraffin slides. Slices of $4 \mu \mathrm{M}$ were cut, stained with hematoxyllin and counterstained with eosin. Measurements of villus height were performed by an automatic interactive videoanalysis system (Jandel video analysis system, Erkath, Germany). The measurements were performed in at least 10 well oriented villi.

- lactulose /Mannitol determinations

Urinary lactulose and mannitol were determined with gas-liquid chromatography as described by Shippee et al ${ }^{1 / 4}$. The excretion percentages of the different sugar probes were determined and from these the excretion ratios of lactulose/mannitol were calculated. 


\section{Statistical analysis}

Data are presented as means \pm standard deviation (SD). The PIW, the FFMI and the PWL were considered to be markers of the nutritional status whereas the erythrocyte sedimentation rate (ESR), leukocyte count and (pre)albumin concentrations were considered to reflect the presence and severity of systemic inflammation 15 .

The relationship between nutritional depletion or the presence of inflammation and plasma and mucosal glutamine concentrations were studied by determining Spearman's correlation coefficients. Similar calculations were performed to establish the effects of nutritional status and inflammation on gut permeability and villus height. Based on the ESR and albumin results, groups were distinguished having either significant or no signs of systemic inflammation. Cut off points were an ESR of $25 \mathrm{~mm} / \mathrm{h}$ and an albumin concentration of 35 gram/liter. Similarly, groups were distinguished that were either depleted or not. Presence or absence of nutritional depletion was defined using the PIW with a cut off point

Table 1. Demographic factors and diagnosis of patients.

\begin{tabular}{|c|c|c|c|}
\hline No & Sex & Age & Diagnosis \\
\hline 1 & $M$ & 67 & Enterocutaneous fistula \\
\hline 2 & $M$ & 77 & Colonic carcinoma \\
\hline 3 & $M$ & 65 & Gastric carcinoma \\
\hline 4 & $F$ & 75 & Colonic carcinoma \\
\hline 5 & $\mathrm{~F}$ & 77 & Colonic carcinoma \\
\hline 6 & $M$ & 36 & Vertical banded gastroplasty \\
\hline 7 & M & 70 & Enterocutaneous fistula \\
\hline 8 & $\mathrm{~F}$ & 77 & lleus \\
\hline 9 & $F$ & 64 & Diverticulitis \\
\hline 10 & $\mathrm{~F}$ & 31 & Pancreatitis \\
\hline 11 & $M$ & 45 & Crohn's disease \\
\hline 12 & $M$ & 43 & Ulcerative colitis \\
\hline 13 & $M$ & 33 & Ulcerative colitis \\
\hline 14 & $F$ & 67 & Crohn's disease \\
\hline 15 & M & 24 & Ulcerative colitis \\
\hline 16 & $\mathrm{~F}$ & 36 & Crohn's disease \\
\hline 17 & M & 40 & Crohn's disease \\
\hline 18 & $F$ & 24 & Crohn's disease \\
\hline 19 & $M$ & 22 & Crohn's disease \\
\hline 20 & $M$ & 21 & Crohn's disease \\
\hline 21 & $F$ & 31 & Crohn's disease \\
\hline 22 & $M$ & 44 & Colonic carcinoma \\
\hline 23 & M & 23 & Colitis (indeterminate) \\
\hline 24 & $F$ & 31 & Crohn's disease \\
\hline 25 & $M$ & 33 & Crohn's disease \\
\hline 26 & $M$ & 78 & Rectal carcinoma \\
\hline
\end{tabular}


of $90 \%$. The same calculations were made with FFMI and PWL (cut off points $90 \%$ and $10 \%$ respectively), but because the results were similar, only the data obtained using the PIW will be presented. Differences of glutamine concentrations, gut permeability and villus height between the groups were tested with Mann Whitney U for significance.

\section{RESULTS}

Twenty six patients were included in the study. Demographic factors and diagnoses are summarized in table 1.

\section{Glutamine levels}

Neither PIW nor the FFMI or PWL were found to correlate with plasma or mucosal glutamine concentrations. On the other hand, the ESR was significantly correlated $(\mathrm{r}=$ $0.578, p<0.01$ ) to plasma glutamine concentrations (see fig 1). In addition, mucosal glutamine concentrations were also significantly correlated to $\operatorname{ESR}(r=-0.612, p=0.01)$ (see fig 2). No significant correlations with indicators of nutritional depletion were found. After division of patients in groups with and without inflammation, using ESR levels, significantly lower plasma glutamine concentrations were found in the groups with inflammatory activity (table 2). This was also true for mucosal glutamine concentrations (table 2). Division of patients in depleted versus not depleted yielded no differences in glutamine concentrations in plasma or in gut mucosa (table 2).

Table 2 Effects of inflammation and nutritional depletion on plasma and mucosal glutamine concentrations (umol/L). Erythrocyte sedimentation rate (ESR) is expressed in $\mathrm{mm} / \mathrm{h}$, albumin concentrations in $g / L$, weight loss in percentage of original weight and fat free mass index (FFMI) in \%. All laboratory values are expressed as means $\pm S D$.

\begin{tabular}{lllll}
\hline & & $n=$ & $\begin{array}{l}\text { Plasma glutamine } \\
(\mu \mathrm{mol} / \mathrm{L})\end{array}$ & $\begin{array}{l}\text { Mucosal glutamine } \\
(\mu \mathrm{mol} / \mathrm{L})\end{array}$ \\
\hline ESR & $<25 \mathrm{~mm} / \mathrm{h}$ & 10 & $663 \pm 176$ & $2897 \pm 1431$ \\
& $>25 \mathrm{~mm} / \mathrm{h}$ & 15 & $558 \pm 169^{*}$ & $2432 \pm 314 \dagger$ \\
Albumin & $p$ & & 0.03 & 0.02 \\
& $>35 \mathrm{~g} / \mathrm{L}$ & 16 & $653 \pm 114$ & $2828 \pm 457$ \\
& $<35 \mathrm{~g} / \mathrm{L}$ & 9 & $559 \pm 160^{*}$ & $2502 \pm 323$ \\
Percentage & $\mathrm{p}$ & & 0.03 & $\mathrm{NS}$ \\
weight loss & $>10 \%$ & 12 & $601 \pm 180$ & $2575 \pm 507$ \\
& $>10 \%$ & 13 & $596 \pm 187$ & $2696 \pm 358$ \\
FFMI & $p$ & & $\mathrm{NS}$ & $\mathrm{NS}$ \\
& $>90 \%$ & 14 & $644 \pm 153$ & $2579 \pm 381$ \\
& $<90 \%$ & 11 & $601 \pm 220$ & $2764 \pm 479$ \\
\hline${ }^{\circ} \mathrm{p}=0.03,+\mathrm{p}=0.02$ & $\mathrm{p}$ & & $\mathrm{NS}$ & $\mathrm{NS}$ \\
\hline
\end{tabular}




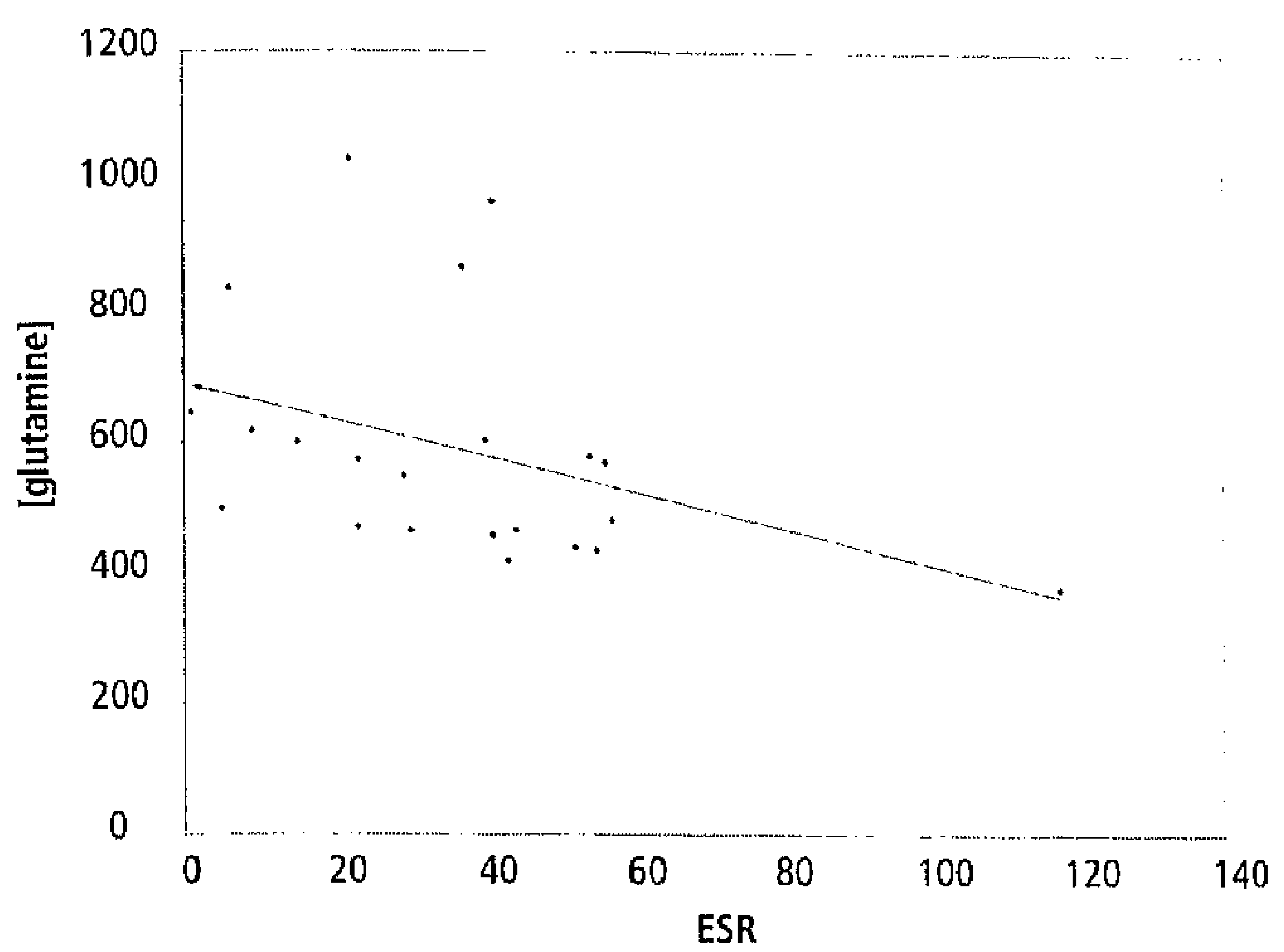

Figure 1 Correlation of ESR $(\mathrm{mm} / \mathrm{h})$ to plasma glutamine concentrations $(\mu \mathrm{mol} / \mathrm{L})$. $r=0.578(p<0.01)$

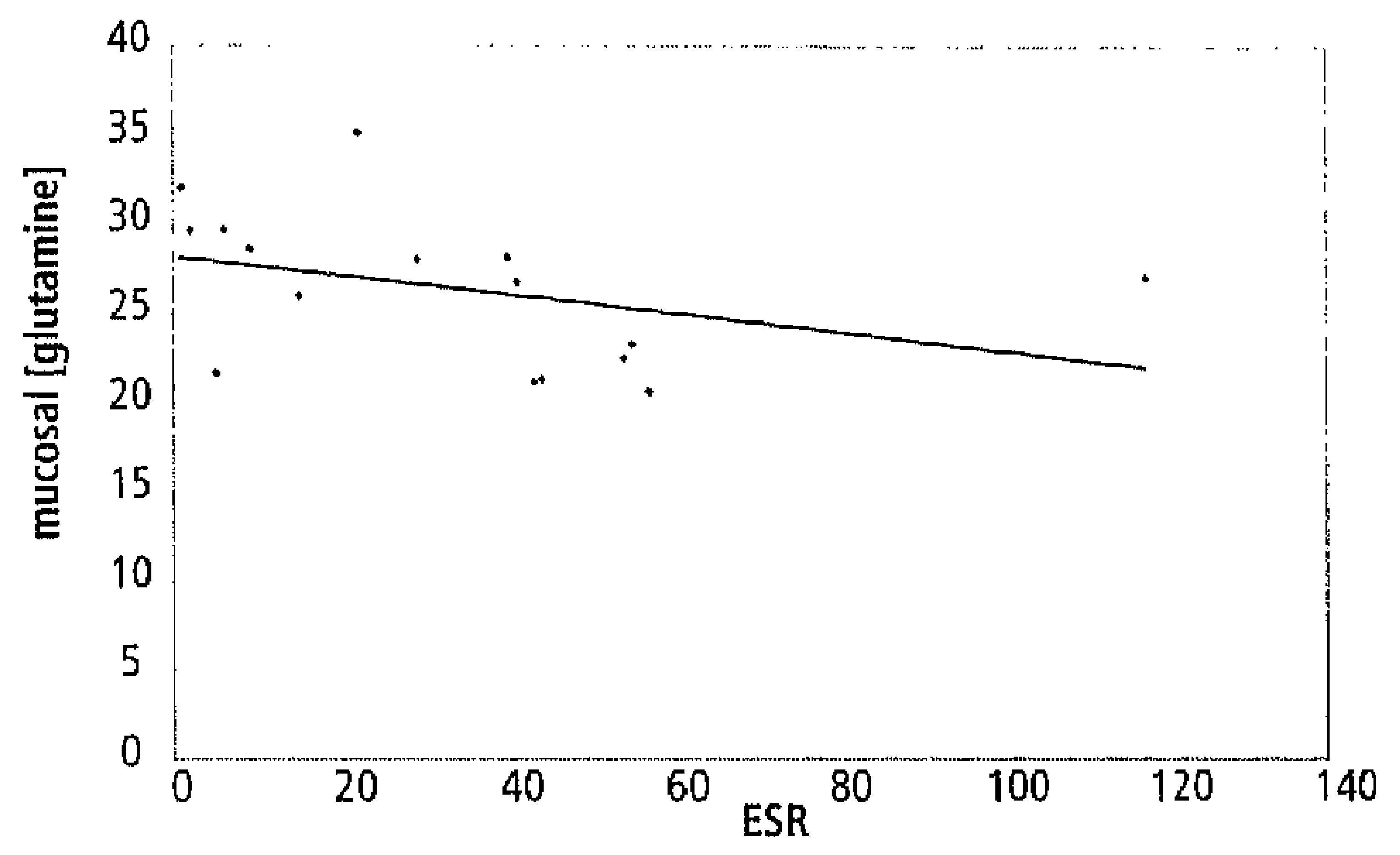

Figure 2 Correlation of ESR $(\mathrm{mm} / \mathrm{h})$ and mucosal glutamine concentration $/ \mathrm{mmol} / \mathrm{L})$.

$$
r=-0.612(p=0.01)
$$

\section{Gut permeability}

Intestinal permeability showed a negative correlation with prealbumin concentrations $(r=$ $-0.601, p<0.01)$. Again, the presence of systemic inflammation resulted in significantly higher intestinal permeability. Nutritional depletion defined by PIW or FFMI did not result in altered permeability values (table 3). A significant loss of body weight resulted in higher $L / M$ ratio's ( 0.20 vs 0.11 ) but this was statistically not significant $(p=0.11)$.

\section{Villus height}

In contrast to the previous parameters, inflammatory activity did not affect villus height. Moreover, the presence of nutritional depletion as defined by a low PIW, or a high PWL 
Table 3 Effects of inflammation and nutritional depletion on lactulose/mannitol ratio. Erythrocyte sedimentation rate (ESR) is expressed in $\mathrm{mm} / \mathrm{h}$, albumin concentrations in $\mathrm{g} / \mathrm{L}$, weight loss in percentage of original weight and fat free mass index (FFMI) in \%. All laboratory values are expressed as means $\pm S D$.

\begin{tabular}{|c|c|c|c|c|c|}
\hline & & L/M ratio & & & L/M ratio \\
\hline \multirow[t]{3}{*}{$\overline{E S R}$} & $\overline{<25}$ & $0.09 \pm 0.07$ & Weight loss & $<10 \%$ & $0.10 \pm 0.07$ \\
\hline & $>25$ & $0.20 \pm 0.18^{*}$ & & $>10 \%$ & $0.21 \pm 0.19$ \\
\hline & $\mathrm{p}$ & 0.03 & & $p$ & NS \\
\hline \multirow[t]{3}{*}{ Albumin } & $>35$ & $0.11 \pm 0.08$ & FFMI & $>90 \%$ & $0.18 \pm 0.23$ \\
\hline & $<35$ & $0.17 \pm 0.18$ & & $<90 \%$ & $0.19 \pm 0.10$ \\
\hline & $p$ & NS & & $p$ & NS \\
\hline
\end{tabular}

similarly did not result in altered values for villus height. However, depletion defined by a FFMI lower than $90 \%$ yielded significantly lower villus height than in non depleted patients $(431.0 \pm 43.8 \mu \mathrm{m}$ versus $489.45 \pm 34.2 \mu \mathrm{m}, p<0.01)$ (table 4 , fig. 3 ).

Table 4 Effects of inflammation and nutritional depletion on villus height ( $\mu M)$. Erythrocyte sedimentation rate (ESR) is expressed in $\mathrm{mm} / \mathrm{h}$, albumin concentrations in $\mathrm{g} / \mathrm{L}$, weight loss in percentage of original weight and fat free mass index (FFMI) in \%. Al

\begin{tabular}{lll}
\hline & & Villus height \\
\hline ESR & $<25$ & $491 \pm 53$ \\
& $>25$ & $437 \pm 56$ \\
Albumin & $p$ & NS \\
& $>35$ & $462 \pm 37$ \\
& $<35$ & $454 \pm 72$ \\
Weight loss & $p$ & NS \\
& $<10 \%$ & $452 \pm 57$ \\
FFMI & $>10 \%$ & $466 \pm 64$ \\
& $\mathrm{P}$ & NS \\
& $>90 \%$ & $489 \pm 34$ \\
& $<90 \%$ & $432 \pm 44^{*}$ \\
\hline
\end{tabular}




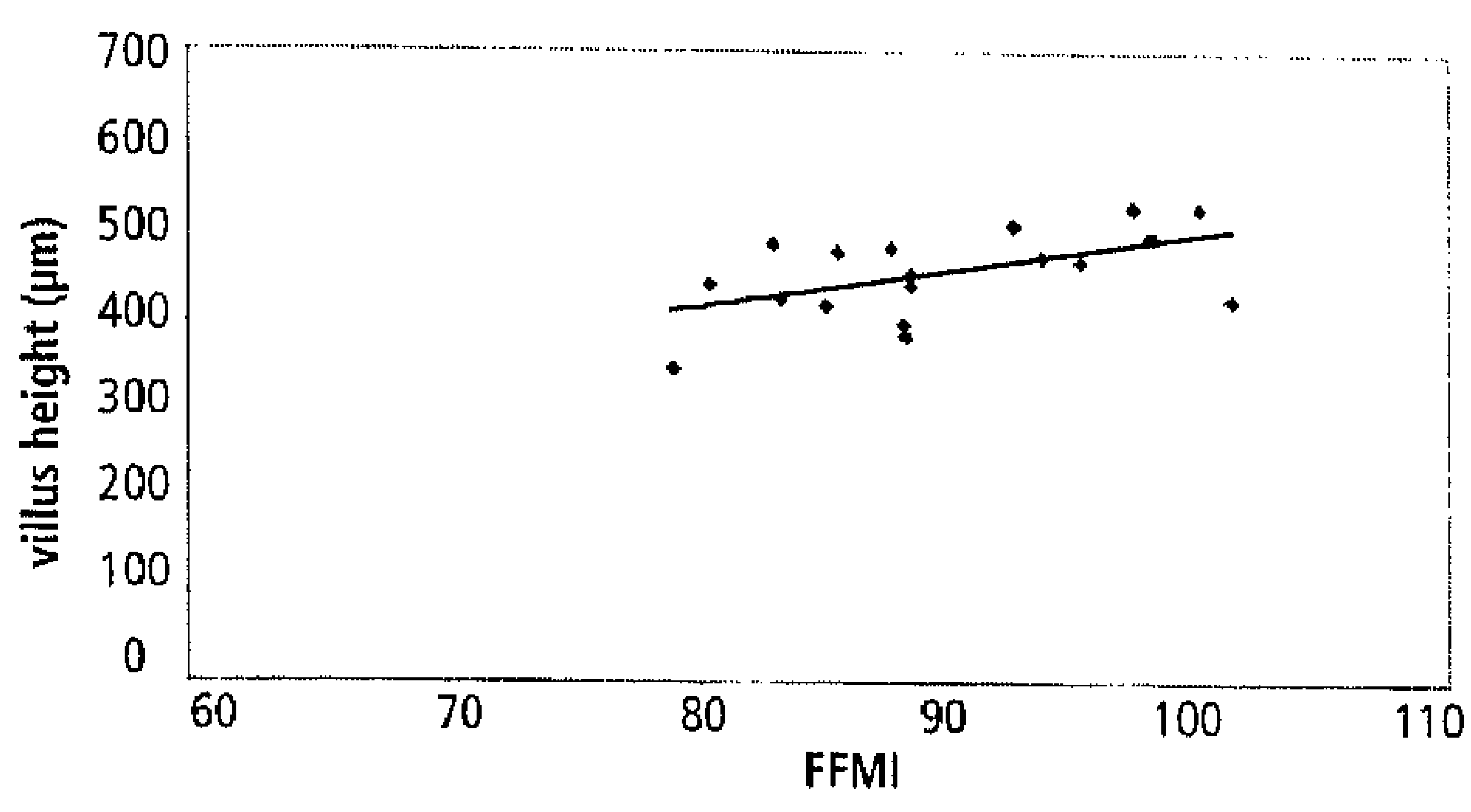

Figure 3 Correlation of FFMI with villus height ( $\mu M)$. $r=0.514(p=0.024)$

\section{Discussion}

The clinical efficacy of glutamine supplementation has been subject of much discussion. Although substantial evidence has been collected in experimental research, clinical studies have produced more confusing data. In this manuscript we try to provide an explanation for the lack of effect of glutamine supplementation in depleted patients ${ }^{6}$, although this population had been hypothesized to be at risk for glutamine depletion ${ }^{16}$.

A major obstacle in distinguishing the effects of nutritional depletion and inflammatory activity concerns the measurement of these variables. No commonly accepted definition of nutritional depletion exists. Frequently factors associated with an increased incidence of complications after major surgery are used. However, many of these parameters are reflections of an active inflammatory response ${ }^{15}$. Moreover, inflammatory stress is frequently present in depleted patients. In this study inflammatory activity was measured by elevated ESR and decreased albumin concentrations, which represent inflammation rather than nutritional status per se. Obviously, these parameters only partially characterize the inflammatory response. Measurement of cytokines, cytokine receptors, other products of visceral protein synthesis, hormones etcetera might have provided a more complete assessment of inflammatory activity. However, the added value of measurement of these parameters can be disputed. Clinically, ESR and albumin concentrations show a precise correlation with (chronic) inflammatory activity. Percentage ideal body weight, fat free mass index and the percentage of weight lost in the previous months were chosen as measures reflecting (changes in) body cell mass since they are accepted parameters of nutritional depletion and have proven to correlate with a high rate of (postoperative) complications in several large studies ${ }^{17-19}$.

Although from a physiological viewpoint endogenous glutamine fluxes are a more obvious endpoint, we only measured glutamine concentrations since net glutamine fluxes are difficult to measure if at all possible ${ }^{20}$. 
In this limited population plasma and mucosal glutamine concentrations as well as gut permeability were consistently correlated with the presence and severity of inflammatory stress in contrast to standard parameters of nutritional depletion. Systemic inflammation profoundly affects (inter)organ glutamine metabolism, resulting in enhanced release by muscle and lung21,22 and increased consumption by other organs $23-27$. Regulatory mechanisms include glutamine synthetase and glutaminase expression and activity, transport activity as well as protein synthesis and catabolism in different organs ${ }^{28,29}$. The underlying orchestrating mechanisms may include changes in glutamine plasma concentrations ${ }^{30}$ or hormonal changes affecting glutaminase or glutamine synthetase expression. Another attractive hypothesis is that in times of severe injury or inflammatory stress, decreases in membrane potential ${ }^{31}$ result in release of glutamine. Endotoxinaemia and inflammatory mediators have been shown to decrease the resting membrane potential ${ }^{32}, 33$. This is the result of marked increases in intracellular sodium and chloride concentrations and a decrease in intracellular potassium ${ }^{31}$. Since transport of glutamine into the cell depends on this membrane potential, this process can lead to a net efflux of glutamine out of the cell into the plasma compartment. This phenomenon can explain the sudden and unalterable decrease of intracellular glutamine seen during conditions of severe inflammatory stress.

The metabolic processes described above are a normal adaptive response but not much is known concerning the consequences of prolonged, severe inflammatory stress or inflammatory stress in a previously nutritionally depleted individual. Wasting of muscle tissue might interfere with muscle glutamine efflux, resulting in glutamine deficiency. Accelerated muscle catabolism, present in most critically ill patients, can result in severe muscle wasting impeding recovery to normal function and possibly decreasing survival ${ }^{34}$. In theory, glutamine supplementation under these circumstances might be beneficial34. However, the current data indicate that nutritional depletion per se does not affect glutamine concentrations nor intestinal permeability. Whether this also applies to critically ill patients is unknown. Interestingly, several studies including patients with significant amounts of inflammatory stress have shown improved outcomes with the use of glutamine supplementation $2,35,30$ although some have not reproduced all of these findings $1,4$.

In summary, current data indicate that under conditions of serious inflammatory stress glutamine (interorgan) metabolism is altered significantly. However, the preliminary evidence provided in our study suggests that depletion of body cell mass per se is not reflected in altered plasma and tissue glutamine concentrations. Because of the limited number of patients and the partial characterization of the inflammatory response these observations should be confirmed in a larger study. Nevertheless, the data provide a potential explanation for the negative results of glutamine supplementation in purely
nutritionally depleted subjects. 


\section{REFERENCES}

1. Schloerb PR, Amare M. Total parenteral nutrition with glutamine in bone marrow transplantation and other clinical applications (a randomized, double-blind study).J Parenter Enteral Nutr 1993: 17:407-13.

2. Ziegler TR, Young LS, Benfell K, et al. Clinical and metabolic efficacy of glutamine-supplemented parenteral nutrition after bone marrow transplantation. A randomized, double-blind, controlled study. Ann Intern Med 1992; 110:821-8.

3. Hulst van der RRWJ, van Kreel BK, Meyenfeldt von MF, et al. Glutamine and the preservation of gut integrity. Lancet 1993; 341:1303-5.

4. Powell-Tuck J, Jamieson CP, Bettany GE, et al. A double blind, randomised, controlled trial of glutamine supplementation in parenteral nutrition. Gut 1999; 45(1):82-8.

5. Buchman AL. Glutamine: is it a conditionally required nutrient for the human gastrointestinal system? I Am Coll Nutr 1990; 15(3):199-205.

o. Hulsewé KWE, van Acker BAC, Hameeteman W, et al. Does glutamine-enriched parenteral nutrition really affect intestinal morphology and gut permeability? Clin Nutr 2004; 23(5):1217.25.

7. Hulst van der RRWJ, Deutz NEP, Meyenfeldt von MF, Eibers JMH, Stockbrügger RW, Soeters PB. Nutritional depletion and mucosal glutamine concentration. Clin Nutrition 1994; 13:228-33.

8. van der Hulst RR, von Meyenfeldt MF, van Kreel BK, et al. Gut permeability, intestinal morphology, and nutritional depletion. Nutrition 1998; 14|11:1-6.

9. Spaeth $G$, Gottwald T, Haas W, Holmer M. Glutamine peptide does not improve gut barrier function and mucosal immunity in total parenteral nutrition. J Parenter Enteral Nutr 1993; 17(4):317-23.

10. Hulsewe KW, Meijerink WJ, Soeters PB, von Meyenfeldt MF. Assessment of outcome of perioperative nutritional interventions. Nutrition 1997; 13(11-12):996-8.

11. Metropolitan Life Insurance Company. New weight standards for men and women. Bulletin Metropolitan Life Insurance Foundation 1983; 04:1-4.

12. Segal KR VIM, Fitzgerald PI, Hodgdon JA, Van Itallie TB. Lean body mass estimation by bioelectrical impedance analysis: a four-site cross-validation study. Am J Clim Nutr 1988; 47:7-14.

13. van Ejjk HM, van der Heijden MA, van Berlo CL, Soeters PB. Fully automated liquid-chromatographic determination of amino acids. Clin Chem 1988; 34(12):2510-3.

14. Shippee RL, Johnson AA, Cioffi WG, Lasko J, LeVoyer TE, Jordan BS. Simultaneous determination of lactulose and mannitol in urine of burn patients by gas-liquid chromatography. Clin Chem 1992; $38|3|: 343.5$.

15. Klein S. The myth of serum albumin as a measure of nutritional status. Gastroenterology 1990; 99:1845-51.

16. Soeters PB. Glutamine: the link between depletion and diminished gut function? J Am Coll Nutr 1996; 15(3):195-0.

17. Studley HO. Percentage weight loss, a basic indicator of surgical risk in patients with chronic peptic ulcer. JAMA 1936; 106:458-60.

18. Perioperative total parenteral nutrition in surgical patients. The Veterans Affairs Total Parenteral Nutrition Cooperative Study Group. N Engl / Med 1991; 325:525-32.

19. Von Meyenfeldt MF, Meijelink WIHJ, Rouflart MMJ, Buil-Maassen MTHJ, Soeters PB. Perioperative nutritional support: a randomised clinical trial. Clin Nutr 1992; 1:180-6.

20. Van Acker BAC, Hulsewé KWE, Wagenmakers AJM, et al. Absence of glutamine isotopic steady state: implications for the assessment of whole-body glutamine production rate. Clin Sci (Colch/ 1998; 95(3):339-46. 
21. Clowes GHA, Randall HT, Cha C-J. Amino acid and energy metabolism in septic and traumatized patients. J Parenter Enteral Nutr 1980; 4(2):195-205.

22. Plumley DA, Souba WW, Hautamaki RD, et al. Accelerated lung amino acid release in hyperdynamic septic surgical patients. Arch Surg 1990; 125(1):57-61.

23. Newsholme EA, Parry Billings M. Properties of glutamine release from muscle and its importance for the immune system. J Parenter Enteral Nutr 1990; 14:63S-7S.

24. Sarantos P, Abouhamze A, Souba WW. Glucocorticoids regulate intestinal glutaminase expression. Surgery $1992 ; 112(2): 278.83$.

25. Deutz NEP, Reijven PL, Athanasas G, Soeters PB. Post-operative changes in hepatic, intestinal, splenic and muscle fluxes of amino acids and ammonia in pigs. Clin Sci Colch 1992; 83(5):607-14.

26. Souba WW, Wilmore DW. Postoperative alteration of arteriovenous exchange of amino acids across the gastrointestinal tract. Surgery 1983; 94(2):342-50.

27. Watford M, Chellaraj V, Ismat A, Brown P, Raman P. Hepatic glutamine metabolism. Nutrition 2002; 18(4):301-3.

28. Karinch AM, Pan M, Lin CM, Strange R, Souba WW. Glutamine metabolism in sepsis and infection. I Nutr 2001; 131 (9 Suppl):2535S-8S.

29. Labow BI, Souba WW, Abcouwer SF. Mechanisms governing the expression of the enzymes of glutamine metabolism-glutaminase and glutamine synthetase. J Nutr 2001; 131 (9 Suppl):2467S-74S.

30. Biolo G, Fleming RY, Maggi SP, Nguyen TT, Herndon DN, Wolfe RR. Inhibition of muscle glutamine formation in hypercatabolic patients. Clin Sci (Lond) 2000; 99(3):189-94.

31. Cunningham JN, Jr., Carter NW, Rector FC, Jr., Seldin DW. Resting transmembrane potential difference of skeletal muscle in normal subjects and severely ill patients. J Clin Invest 1971; 50(1):49-59.

32. Tracey KJ, Lowry SF, Beutler B, Cerami A, Albert JD, Shires GT. Cachectin/tumor necrosis factor mediates changes of skeletal muscle plasma membrane potential. J Exp Med 1986; 104(4):1368-73.

33. Gibson WH, Cook JI, Gatipon G, Moses ME. Effect of endotoxin shock on skeletal muscle cell membrane potential. Surgery 1977; 81(5):571-7.

34. Griffiths RD. Muscle mass, survival, and the elderly ICU patient. Nutrition 1996; 12(6):456-8.

35. Griffiths RD, Jones C, Palmer A. Six-month outcome of critically ill patients given glutaminesupplemented parenteral nutrition. Nutrition 1997; 13(4):295-302.

36. Houdik APJ, Rijnsburger ER, Jansen J, et al. Randomised trial of glutamine-enriched enteral nutrition on infectious morbidity in patients with multiple trauma. Lancet 1998; 352(9130):772-6. 
Inflammation versus nutritional depletion and glutamine metabolism 


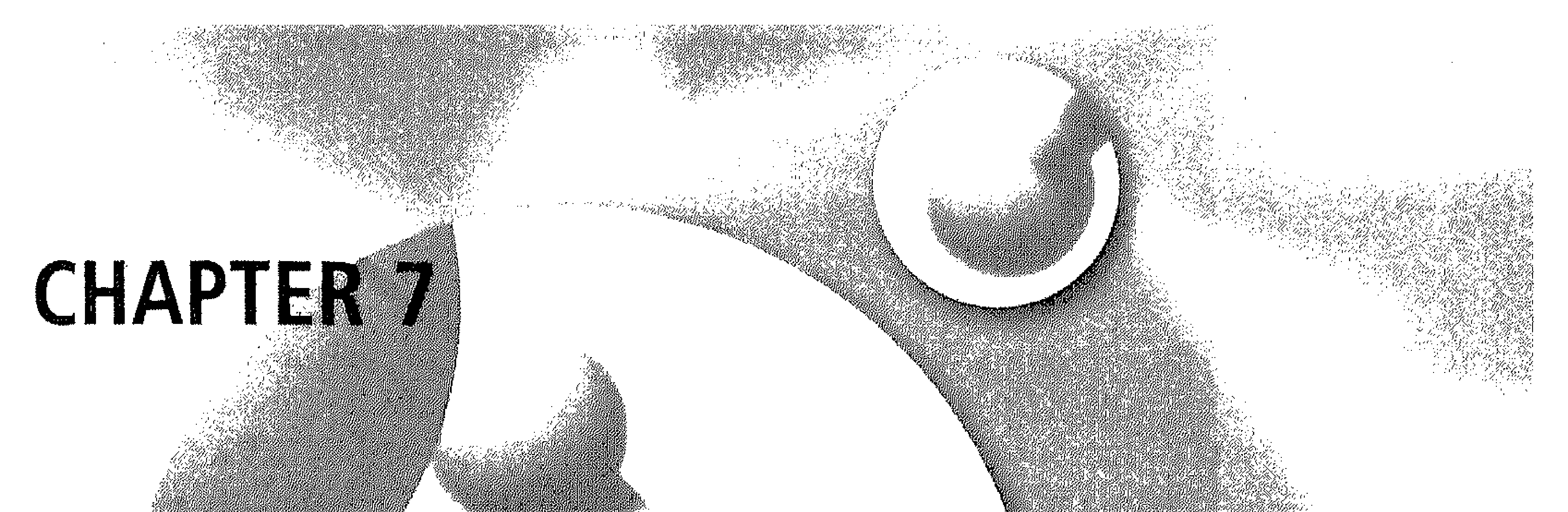

Effects of glutamine supplementation on intestinal histology and gut barrier function in depleted patients

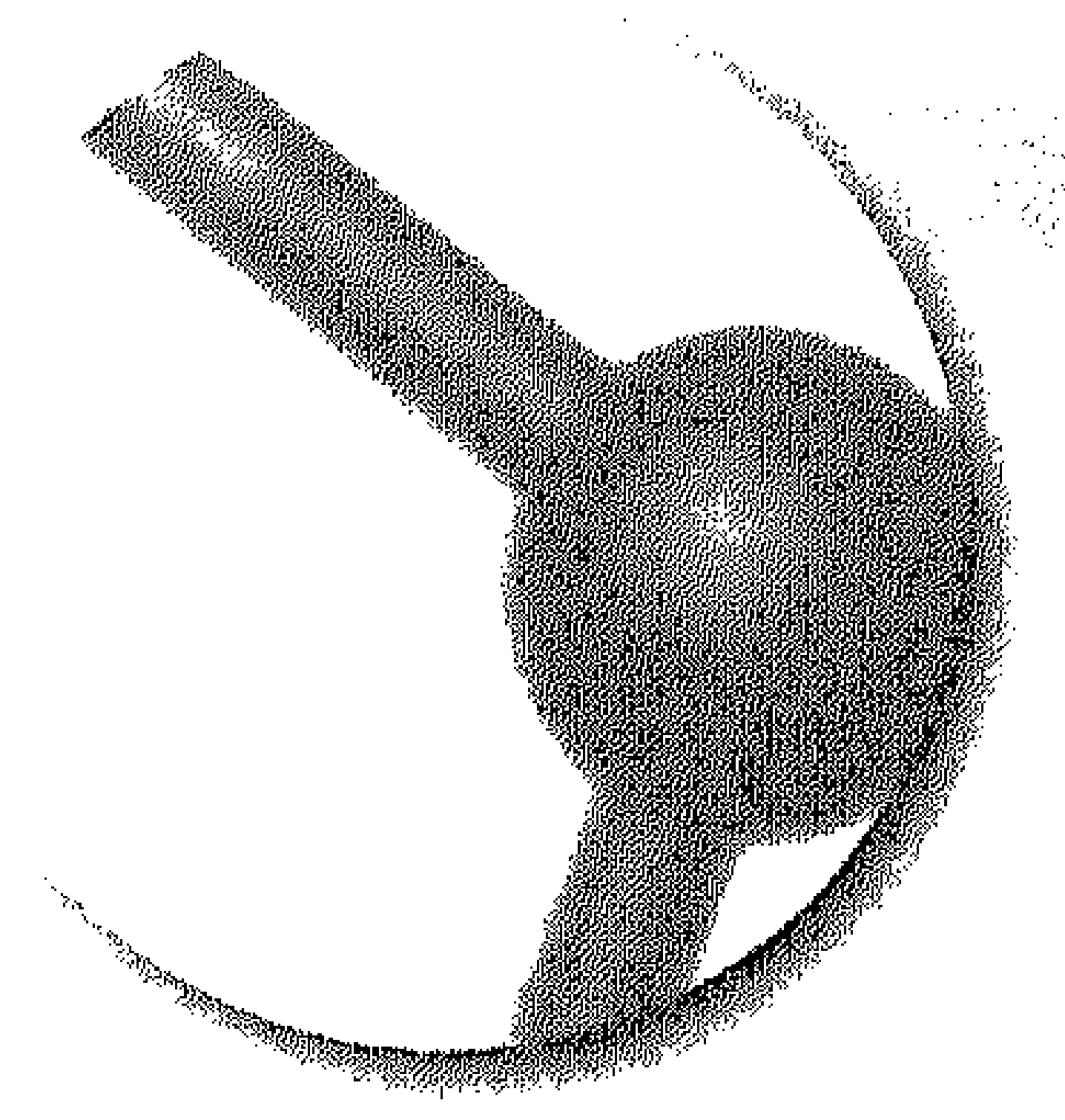

Karel W.E. Hulsewé, Bernadette A.C. van Acker, ${ }^{1}$ Wim Hameeteman, ${ }^{2}$ Rene R.W.J. van der Hulst, Tryfon Vainas, 3Jan-Willem Arends, ${ }^{4}$ Bernard K. van Kreel, Maarten F. von Meyenfeldt, Peter B. Soeters

Departments of General Surgery, 'Gastroenterology, 2'lastic and Reconstructive Surgery, ${ }^{3}$ Pathology and "Clinical Chemistry, University Hospital Maastricht, The Netherlands Published in Clinical Nutrition 2004; 23: 1217-1225 


\section{ABStRact}

\section{Background}

Nutritional depletion has been related to low glutamine levels in plasma and gut mucosa. This study was set up to investigate the effects of glutamine-enriched total parenteral nutrition on
intestinal morphology and permeability.

\section{Methods}

Twenty-three depleted patients were randomized and after stabilization baseline measurements were performed. Plasma glutamine concentrations, gut morphology (including proliferation and lymphocyte markers) and intestinal permeability were measured. After administration of 8.10 days of a glutamine enriched total parenteral nutrition or an isonitrogenous control solution the measurements were repeated.

\section{Results}

No significant changes in glutamine concentrations, intestinal permeability, mucosal morphology or gut mucosal inflammation were observed between groups.

\section{Conclusions}

Glutamine enriched total parenteral nutrition in a depleted patient population does not result in improvements in gut morphology and gut barrier function. 


\section{INTRODUCTION}

Already in the seventies it was known that glutamine serves important functions for the gastrointestinal tract, which consumes glutamine in large quantities ${ }^{1}$. The adverse effects of parenteral nutrition on the gut, which received much attention in the last decade, have been attributed, in part, to the absence of glutamine in total parenteral nutrition (TPN)2, 3. Glutamine has been administered intravenously, but the low solubility and instability of free glutamine has precluded its wide usage. These practical problems led to the development of glutaminedipeptides, such as glycyl-glutamine or alanyl-glutamine, which were more soluble and stable than free glutamine, enabling addition of glutamine to total parenteral nutrition (TPN) ${ }^{4}$. Since then many clinical studies have appeared investigating the clinical effects of glutamine.

Studies investigating the effects of glutamine-enriched TPN on the intestinal mucosa have yielded conflicting results. While multiple studies have demonstrated beneficial effects of glutamine on gut morphology and barrier function in animal models, in healthy volunteers and in a patient population ${ }^{5-9}$, others were not able to repeat these findings ${ }^{10}$. Moreover, studies in rodents have shown considerably larger effects of TPN and glutamine supplementation in comparison to clinical studies 11,12 .

There are a few additional explanations for these conflicting results: not all patients included in the studies may be lacking adequate endogenous glutamine stores, i.e. glutamine may not be considered essential in these subjects, thereby reducing the effects of its supplementation. Furthermore, differences in baseline nutritional status may exist between groups especially if baseline samples are taken without allowing for a standardized stabilization period: some may be nutritionally depleted with or without micronutrient deficiencies, whereas others may have been eating until inclusion in the study.

Because of these confounding factors we designed a randomized trial in order to include a homogeneous patient population, and to allow for a short stabilization period during which all subjects were fed a hypocaloric, glutamine-free parenteral nutrition solution. This was done in order to make the conditions during baseline measurements more comparable between subjects and more comparable to the conditions during the measurements at the end of the study.

Nutritional depletion has been associated with low plasma and mucosal glutamine concentrations, suggesting a state of glutamine deficiency ${ }^{13}$. The endogenous glutamine production rate, however, has not been measured in such a population. Furthermore, depletion has been associated with mucosal atrophy and increased intestinal permeability 13,14 .

On the basis of these considerations we hypothesized that nutritionally depleted patients are likely to benefit from glutamine enrichment of TPN 15 .

The aim of this study was therefore to investigate the effects of glutamine enrichment of TPN on the plasma glutamine concentrations as well as its effects on intestinal morphology and permeability in depleted patients. 


\section{Patients and methods}

\section{Patients}

Patients admitted for gastrointestinal surgery were eligible for the study if they were nutritionally depleted and required preoperative parenteral nutrition. Nutritional depletion was defined as an involuntary loss of more than $10 \%$ of body weight (BW) within the Metropolitan Life Insurance tables 10 . Furtherm the ideal body weight calculated using the If there were any clinica hypothermia $\left(<36^{\circ} \mathrm{C}\right)$, evidence of sepsis or inolic stress, such as fever $\left(>38.5^{\circ} \mathrm{C}\right)$, excluded. Other exclusion criteria were the intra-abdominal abscesses, patients were pregnancy, insulin dependent diabetes mence of renal or hepatic insufficiency,

Table 1 Demographic data of patients

\begin{tabular}{|c|c|c|c|c|c|c|}
\hline Subject & TPN & Sex & $\overline{\text { Age }}$ & PIW & Loss of BW $(\%)$ & Diagnosis \\
\hline$\overline{103}$ & GT & $m$ & 36 & 88 & 12 & Crohn's disease \\
\hline 106 & GT & $f$ & 46 & 71 & 18 & gastrocolic fistula \\
\hline 108 & GT & $m$ & 65 & 102 & 17 & sigmoid stenosis \\
\hline 111 & GT & $\mathrm{m}$ & 32 & 95 & 25 & chronic pancreatitis \\
\hline 112 & GT & m & 79 & 97 & 13 & colonic stenosis \\
\hline 114 & $\mathrm{Gr}$ & $f$ & 40 & 93 & 16 & tuboovarian abscess \\
\hline 201 & GT & $f$ & 75 & 92 & 18 & rectosigmoid carcinoma \\
\hline 202 & GT & $f$ & 65 & 68 & 10 & rectosigmoid carcinoma \\
\hline 206 & GT & $f$ & 69 & 94 & 14 & gastric carcinoma \\
\hline 207 & GT & $m$ & 68 & 82 & 10 & gastric carcinoma \\
\hline 210 & GT & m & 70 & 115 & 34 & colonic carcinoma \\
\hline 101 & $S T$ & m & 53 & 111 & 4 & chronic pancreatitis \\
\hline 104 & ST & $\mathrm{m}$ & 33 & 91 & 14 & stenosis distal ileum \\
\hline 105 & ST & $m$ & 34 & 76 & 8 & Crohn's disease \\
\hline 107 & $S T$ & $\mathrm{~m}$ & 45 & 83 & 26 & chronic pancreatitis \\
\hline 109 & ST & $f$ & 61 & 100 & 17 & Crohn's disease \\
\hline 110 & $S T$ & $m$ & 60 & 101 & 13 & colonic stenosis \\
\hline 113 & ST & $f$ & 65 & 86 & 11 & Crohn's disease \\
\hline 203 & ST & $f$ & 70 & 90 & 10 & colonic carcinoma \\
\hline 204 & $S T$ & $m$ & 64 & 83 & 15 & colonic carcinoma \\
\hline 205 & ST & $\mathrm{m}$ & 47 & 101 & 14 & gastric carcinoma \\
\hline 208 & ST & $f$ & 77 & 104 & 10 & colonic carcinoma \\
\hline 209 & $S T$ & $m$ & 64 & 108 & 22 & rectosigmoid carcinoma \\
\hline 211 & ST & $m$ & 73 & 82 & 21 & rectosigmoid carcinoma \\
\hline
\end{tabular}


nutrition within 14 days prior to inclusion in the study. The demographic characteristics and diseases of the patients are summarized in table 1.

All participants gave informed consent prior to inclusion in the study. The study was approved by the Medical Ethics Committee of the Academic Hospital Maastricht.

\section{Study design}

After inclusion in the study, the patients were randomized to receive either a glycylglutamine supplemented TPN solution (GT) based on Glamin ${ }^{\circledR}$ (Fresenius Kabi) or an isonitrogenous control solution (CT) based on Vamin 18EF® (Fresenius Kabi). Only the pharmacist was not blinded with regard to the TPN solution given. The composition of the amino acid solutions is described in table 2 . The amino acid solution was mixed with a glucose 50\% solution and a lipid solution (Intralipid 20\%, Pharmacia \& Upjohn, Sweden), each providing approximately $50 \%$ of the calories administered. The total energy expenditure was estimated using the Harris and Benedict formula for basal energy expenditure based on

Table 2 Composition of aminoacid solutions

\begin{tabular}{lll}
\hline Aminoacids & GT & ST \\
\hline Isoleucine & 1.6 & 2.0 \\
Leucine & 2.2 & 2.8 \\
Lysine & 2.6 & 3.2 \\
Methionine & 1.6 & 2.0 \\
Phenilalanine & 1.7 & 2.8 \\
Tyrosine & 0.7 & 0.1 \\
Threonine & 1.6 & 2.0 \\
Tryptophan & 0.5 & 0.7 \\
Valine & 2.1 & 2.6 \\
Alanine & 4.6 & 5.7 \\
Arginine & 3.2 & 4.0 \\
Asparagine & 1.0 & 1.2 \\
Glutamate & 1.6 & 2.0 \\
Histidine & 2.0 & 2.4 \\
Proline & 2.0 & 2.4 \\
Serine & 1.3 & 1.6 \\
Glycine & 3.2 & 2.8 \\
Glutamine & 5.7 & 0.0 \\
Total & & \\
Aminoacids & & \\
Nitrogen & 40.2 & 41.0 \\
\hline
\end{tabular}

Expressed as grams of amino acids per $1000 \mathrm{ml}$ total parenteral nutrition solution. Nitrogen content is expressed in gh. GT is glutamine enriched solution and $C T$ is the control solution 
the actual weight and increased with $40 \%$ for total energy expenditure 17 . The nitrogen $/ \mathrm{kcal}$ ratio averaged 1:150. During the study the patients were not allowed to eat or drink except for $500 \mathrm{ml}$ of water per day. Body composition was determined at baseline with the tracer dilution method using bromide and deuterated water for extracellular and total water space respectively ${ }^{18}$. Ideal body weight was determined by measuring body length and wrist circumference and using the Metropolitan Life Insurance tables ${ }^{10}$.

Beginning at 18.00 hours, an amino acid solution lacking glutamine with glucose was infused continuously, providing $50 \%$ of the calculated energy needs. Since these patients were nutritionally depleted, we gradually increased the TPN administered in order to prevent the occurrence of the refeeding syndrome. After 38 hours baseline measurements were performed: an intravenous line for stable isotope infusion and an arterial catheter in the radial artery for blood sampling was inserted to measure whole body protein turnover, arterial plasma glutamine concentration and glutamine production. The results of these measurements have been published elsewhere ${ }^{19}$. Blood for glutamine concentration was sampled in heparinized tubes chilled on ice. These were centrifuged at $2.200 \mathrm{~g}$ at $4^{\circ} \mathrm{C}$ during 5 minutes. The plasma was deproteinized with $5 \%$ sulfosalicylic acid, mixed and frozen in liquid nitrogen and stored at $-80^{\circ} \mathrm{C}$ until analysis. In addition, after voiding, a gastroduodenoscopy was performed and biopsies were taken of the descending part of the duodenum. Patients were offered sedation with midazolam $5 \mathrm{mg}$ i.v. during this procedure. Three biopsies were fixated in $4 \%$ formaldehyde. One biopsy was snap frozen in thiopental and stored at $-80^{\circ} \mathrm{C}$ until further analysis. Following this procedure a small catheter was passed under endoscopic guidance into the small bowel distal to the site of the biopsies. A sugar tracer solution containing $10 \mathrm{~g}$ lactulose, $0.5 \mathrm{~g}$ mannitol and $1.0 \mathrm{~g}$ rhamnose dissolved in $65 \mathrm{ml}$ of water was infused through this catheter. During the next 6 hours all urine was collected and preserved with thymol, in order to prevent bacterial breakdown of the sugars. After baseline measurements, the patients received the glutamine containing or the control solution in accordance with the randomization. The third day the amount infused equaled $75 \%$ of the total energy expenditure (TEE), and from the 4th day until the end of the study $100 \%$ of the TEE was administered. After 8-10 days of infusion of the study solutions, the measurements described above were repeated.

\section{Outcome measurements}

The primary outcome measure of this study was the lactulose/mannitol ratio as a measure for gut barrier integrity. Since mannitol is not an ideal sugar probe because it can be found in blank urine samples, we included the rhamnose/lactulose ratio as an alternative measure for intestinal permeability. Other secondary outcomes were arterial glutamine concentration, gut mucosal morphology (i.e. crypt depth, villus height and total mucosal thickness), intraepithelial lymphocytes and the proportion of proliferating enterocytes.

\section{Analyses}

- Plasma glutamine concentration

Aminoacid concentrations were determined in arterial plasma using a fully automated high pressure liquid chromatography method as described previously20.

- Morphology/immunohistochemistry 
After fixation in $4 \%$ formaldehyde 3 mucosal biopsies were dehydrated in increasing concentrations of ethanol and embedded in paraffin. Slices of $4 \mu \mathrm{M}$ were cut and stained with haematoxylin and counterstained with eosin. Measurements of villus height and crypt depth were performed by one of the authors $(\mathrm{KH})$ in blinded sections, without any knowledge of the corresponding subject or time in relation to the intervention. For the morphometric measurements, an automatic interactive video-analysis system (Jandel video analysis system, Erkath, Germany) was used. The measurements were performed in at least 10 well oriented villi since this number has been described to result in reliable values ${ }^{14}$.

Additional slices of $3 \mu \mathrm{M}$ were cut for immunohistochemical staining with anti MIB-1, and anti CD-3 antibodies. In short, the slices were deparafinized and put in methanol with hydrogenperoxide to eliminate endogenous peroxidase activity. Hereafter the material was boiled in a citrate buffer using a microwave oven. After incubation with the primary antiserum, rat-anti-mouse biotin was added. The biotin was bound to horse radish peroxidase which was finally coloured by adding diaminobenzidine. Labeling of MIB-1 occurs in replicating enterocytes within the crypt and is expressed as a percentage of all crypt cells. At least 10 well oriented crypts were counted. Anti-CD3 antibodies identify intraepithelial lymphocytes. At least 1000 enterocytes were counted and the figures found are expressed per 100 enterocytes. Positive and negative controls were included in all of these series.

- Lactulose /Mannitol/Rhamnose

Urinary lactulose, mannitol and rhamnose were determined with gas-liquid chromatography as described by Shippee et al.21.

The excretion percentages of the different sugar probes were determined and from these the excretion ratios of lactulose/mannitol and lactulose/rhamnose were calculated.

\section{Statistics}

- Sample size calculation

In order to minimize the number of patients required, we used a sequential design analysis. Unlike the traditional fixed sample design, the sample size in this study was a random variable. The confirmatory comparison of the treatment groups was performed by means of the sequential double triangular procedure proposed by Whitehead 22 . This test procedure will always lead to a confirmatory result stating either significant differences or significant equivalence between the compared treatments.

Based on a previous study from our group 5 a coefficient of variation of the change in lactulose/mannitol ratios of 100\% was assumed. With a type 1 error rate of 0.05 and a type 2 error rate of 0.2 , an average sample number of 11 patients per group was calculated.

All patients completing the study except one were included in the statistical analysis as described in the results section. All data are presented as mean \pm standard error of the mean. For calculation of differences between groups and between baseline and last measurements, the t-test or the Mann-Whitney-U test was used as appropriate. 


\section{RESULTS}

Twenty-four patients were included in the study and randomized to receive either the GT or the CT solution. The patients were fed parenterally because of intolerance to full enteral nutrition. In the cases of inflammatory bowel disease and gastrointestinal malignancy this was due to (partial) obstruction and in the case of the patient with chronic pancreatitis, this was due to pain. The study was terminated prematurely in three patients ( 2 of the GT and 1 of the CT group). Two patients developed symptoms requiring emergency surgery (profuse rectal bleeding in the presence of a rectal malignancy and spiking fever with abdominal pain respectively) and one patient developed a generalized erythema with pruritus in the absence of a clear etiologic factor apart from the parenteral nutrition. This last patient was receiving the standard glutamine free CT solution. One other patient refused to undergo intravascular catheterization and the gastroduodenoscopy on the last study day but gave consent for the measurement of intestinal permeability at this time point. Patient 206 withdrew her consent prior to baseline measurements. Finally one patient was excluded from analysis because of significant disturbances in glutamine and ammonia metabolism due to portal hypertension which was unknown at the time of inclusion in the study.

The percentage of ideal body weight of all included patients was $93 \pm 11 \%$ (mean \pm SD) whereas they had lost $15.4 \pm 6.7 \%$ (mean \pm SD) of their body weight in the 6 months preceding hospital admission. Data of the body composition at baseline are presented in figure 1. Total body water (TBW) was $36.9 \mathrm{~L}$ in the GT group and $35.0 \mathrm{~L}$ in the ST group. Intracellular water made up 57\% of TBW in GT and $49 \%$ in the ST group. None of these differences were statistically significant.

Baseline plasma glutamine concentrations were $609.97 \pm 36.74$ and $562.45 \pm 23.12$ $\mu \mathrm{mol} / \mathrm{L}$ for the GT and ST group respectively (NS). After 8-10 days of administration of the

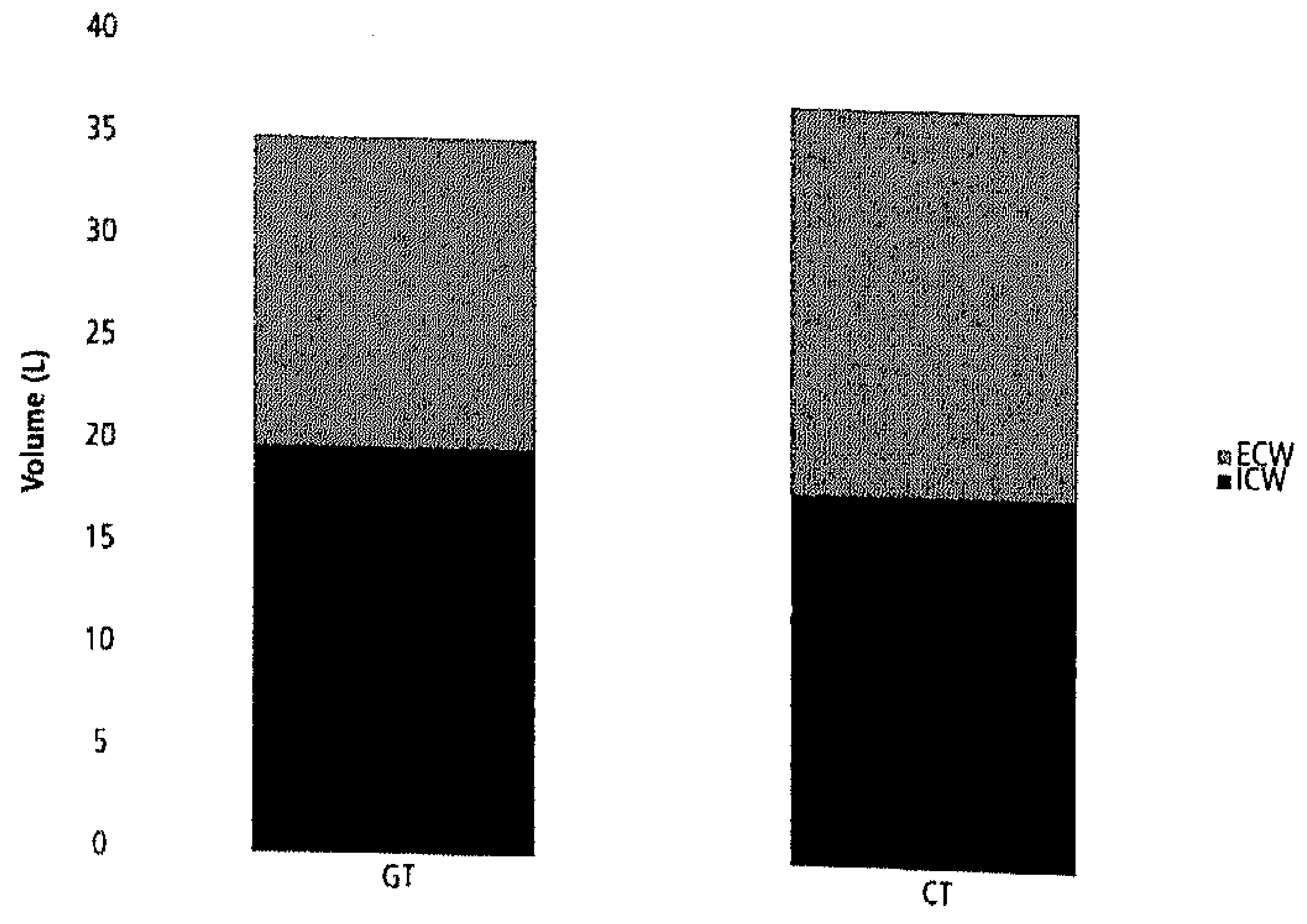

Figure 1 lindvompusition at base'iline in liters.

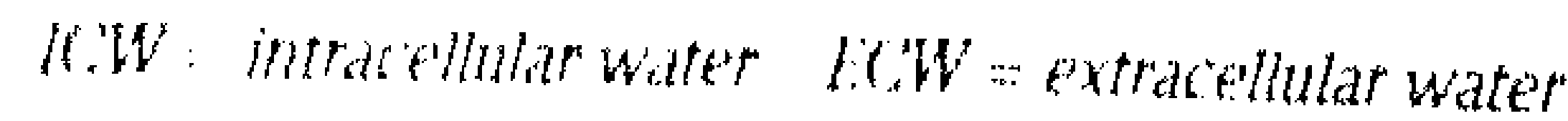


Table 3 Recovery rates of lactulose, mannitol and thamnose (mean \pm SEM). CT is the control group and GT is the glutamine enriched group.

\begin{tabular}{lcccc}
\hline & \multicolumn{2}{c}{ CT } & \multicolumn{2}{c}{ GT } \\
& Baseline & End of study & Baseline & End of study \\
\hline Lactulose recovery rate (\%) & $1.01 \pm 0.6$ & $0.75 \pm 0.2$ & $0.79 \pm 0.3$ & $1.83 \pm 0.8$ \\
Mannitol recovery rate (\%) & $7.50 \pm 0.8$ & $6.40 \pm 0.7$ & $9.96 \pm 1.8$ & $10.99 \pm 1.6$ \\
Rhamnose recovery rate $(\%)$ & $3.80 \pm 0.4$ & $2.93 \pm 0.3$ & $4.05 \pm 0.6$ & $4.83 \pm 0.6$ \\
\hline
\end{tabular}
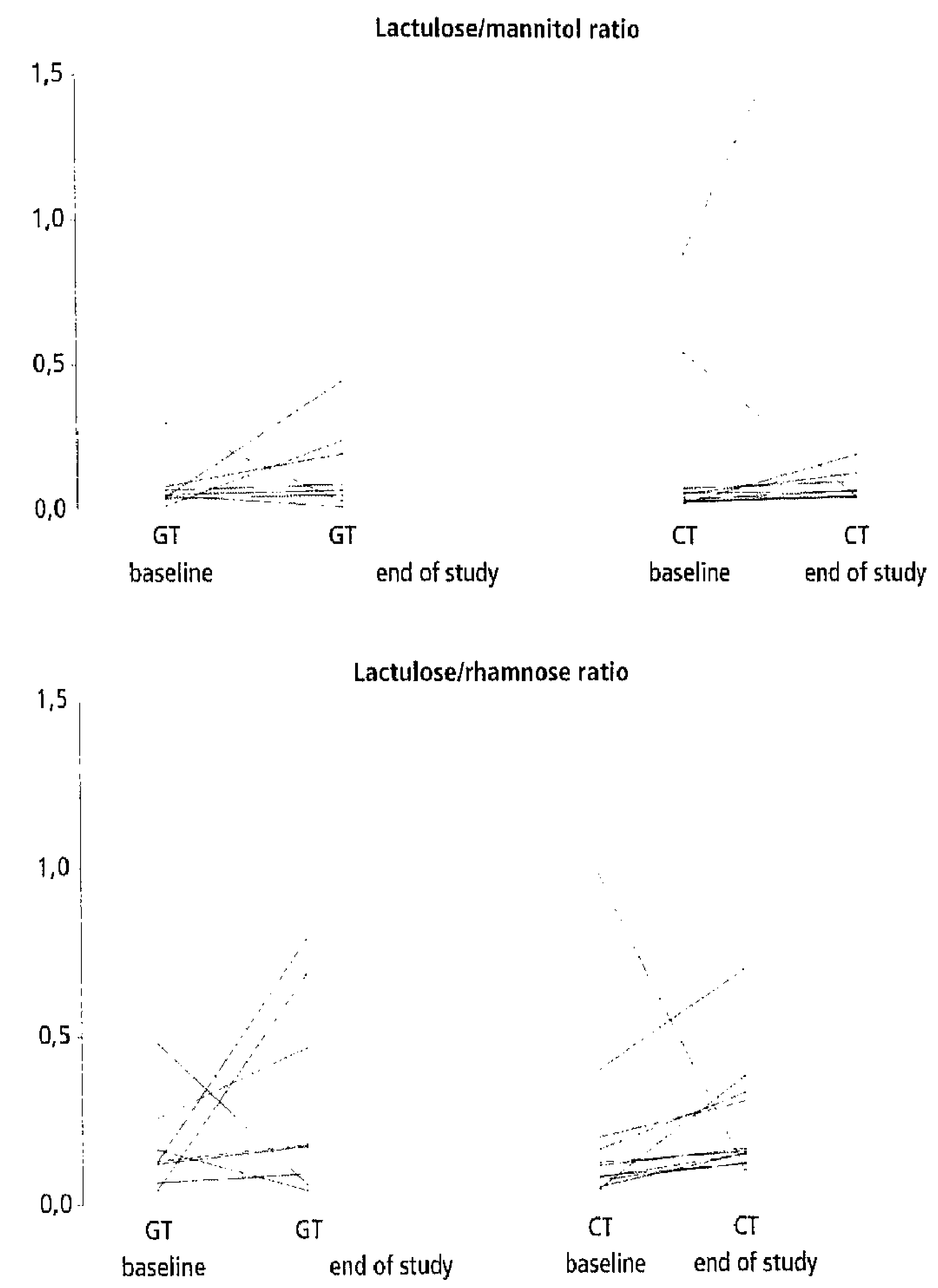

Figure 2 Lactulose/mannitol and lactulose /rhamnose ratios. Individual data are shown. GT is the glutamine enriched study group and CT is the control group. 
study solution, these figures did not change significantly: $641.41 \pm 39.15$ (GT) and 591.79 \pm 35.91 (ST). Lactulose, mannitol and rhamnose recoveries are summarized in table 3. Neither the values at baseline nor the values at the end of the study were significantly different between both groups. The lactulose/mannitol ratio and the lactulose/rhamnose ratios are graphically displayed in fig. 2 . The differences observed between baseline and the last study day were also not significantly different between groups.

Table 4 Histological and immunohistochemical results. Data are presented as mean \pm SEM. CT refers to the control group and GT to the glutamine enriched group. Distances are expressed in $\mu M$. MIB-1 is a marker for enterocyte proliferation and is expressed as a percentage of positive cells in the crypths. IEL are intraepithelial leucocytes and are expressed per 100 enterocytes.

\begin{tabular}{lccccc}
\hline & CT & GT & & & \\
& Baseline & End of study & Baseline & End of study & $p$ \\
\hline Villus height & $488.9 \pm 28.7$ & $477.5 \pm 22.4$ & $462.1 \pm 22.0$ & $463.8 \pm 28.2$ & n.S. \\
Crypt depth & $116.9 \pm 3.3$ & $114.3 \pm 9.1$ & $113.1 \pm 4.5$ & $113.3 \pm 8.2$ & n.s. \\
Mucosal thickness & $605.9 \pm 25.3$ & $591.7 \pm 23.5$ & $575.2 \pm 18.9$ & $577.2 \pm 35.6$ & n.S. \\
MIB-1 & $38.5 \pm 3.6$ & $35.7 \pm 2.0$ & $35.4 \pm 2.1$ & $32.5 \pm 1.7$ & n.s. \\
IEL & $20.9 \pm 2.2$ & $18.2 \pm 1.5$ & $21.2 \pm 2.1$ & $20.1 \pm 2.7$ & n.S. \\
\hline
\end{tabular}

The results of measurements of villus height, crypt depth, total mucosal thickness are described in table 4. In addition, the results of the measurement of MIB-1 and CD-3 positive intramucosal cells can be found in the same table. Statistical analysis of these variables showed no differences between or within groups.

\section{Discussion}

In this study we found no effects of addition of glutamine to total parenteral nutrition on gut mucosal morphology, mucosal inflammatory parameters and intestinal permeability to a sugar probe solution. In addition no effects on plasma glutamine concentrations could be detected. This is in contrast to results from our previous studies 5,23 . There are a few possible factors which may help to explain these differences.

The crucial issue is whether glutamine is an essential amino acid in nutritional depletion or not. In contrast to the previous studies where depleted patients were found to have lowered glutamine plasma concentrations, normal values were found in our current study despite the fact that in the current study all subjects included were nutritionally depleted. The degree of nutritional depletion expressed as percentage ideal body weight (PIW) or recent weight loss was comparable between the current and previous studies, suggesting that another important variable may play a role. Moreover, although 1 patient was unjustly included in the study because he did not fulfill the criteria of depletion (patient 101), we included his data in the analysis. However, recalculation of the data without this particular 
patient gave similar results. Two important differences in patient population between this and our previous study were the inclusion of more cancer patients in the present and more patients with active Crohn's disease and ulcerative colitis in the previous study. Furthermore, the current study excluded specifically patients with signs of clinically significant inflammation, whereas these were included in the former study. Since glutamine levels are also influenced by inflammatory activity 24,25 , this may have been a confounding variable in the correlation of depletion with low plasma glutamine concentrations. Although the glutamine plasma concentrations did not rise after glutamine administration, as has been observed before ${ }^{26}$, we did find increases in total glutamine rate of appearance ${ }^{19}$. However, the concept of glutamine as a conditionally essential amino acid remains to be supported or refuted by demonstrating differences in net glutamine production rates by peripheral tissues like muscle, and subsequently in differences in net utilization by central tissues like the liver and the immune system. We have described the absence of steady state conditions during infusions of isotopically labeled glutamine for up to 11 hours ${ }^{27}$. The effect of depletion on net glutamine production and utilization rates remain therefore to be determined 27 . A second important difference between this and other studies is the fact that in former studies baseline samples were taken after an overnight fast whereas in the present study baseline samples were taken after approximately 38 hours of infusion of a hypocaloric amino acid glucose solution. Our aim was to study the effects of addition of glutamine to TPN. Therefore we tried to make baseline measurements as comparable as possible with the final measurement conditions. Furthermore, this stabilization period also allowed for a better equalization of conditions within and between both groups. On the basis of our data we cannot exclude that infusion of amino acids (and especially the branched chain amino acids) led to increases in glutamine synthesis in our patients in the first $11 / 2$ day resulting in normal plasma glutamine concentrations. If this would be the case, then the rationale for glutamine supplementation must be questioned.

The absence of changes in villus height is in line with the absence of changes in gut permeability. Previous papers have described the simultaneous occurrence of increased permeability and decreases in villus height during administration of TPN5,6, although these phenomena were statistically not related. Buchman et al. determined the change in villus height to be the result of hypoplasia ${ }^{6}$. Another factor involved may be the effect of the infusion of hypocaloric TPN prior to baseline measurements. In a different study we measured the short-term effects of enteral feeding on villus morphology in healthy volunteers. We found a significant increase in villus height within two hours 28 . Therefore changes in mucosal architecture occur rapidly and most likely significant decreases in villus height will have occurred within 36 hours of TPN. This would explain the absence of any changes in villus morphology in this study. However, it is unlikely that this alone would affect gut barrier function since permeability is probably determined by multiple factors rather than by changes in mucosal architecture alone ${ }^{29}$.

The same could apply to the absence of changes in IEL's: increases in intestinal permeability and intestinal atrophy could have resulted in an increased inflammatory activity within the gut. Since this is not the case, no changes in IEL are to be expected. Similarly Buchman et al. found no significant changes in mucosal lymphocytes after administration of TPN in healthy volunteers ${ }^{30}$. In contrast, van der Hulst et al. found a 
decrease in IEL after administration of glutamine enriched TPN, associated with decreased gut permeability 23 .

Finally it is questionable, whether gut barrier function can be tested reliably with a sugar probe solution. Although intestinal barrier function is frequently tested with sugar probe solutions, its clinical relevance remains disputed ${ }^{31}$. These sugar probes are proposed to pass transcellularly or paracellularly respectively and their excretion ratio is a measure of the paracellular transport (which for larger molecules such as lactulose is normally low). However, the gut barrier is composed of several other parts, including the normal gut flora, mucus with several enzymes (e.g. lysozyme), secretory IgA, the gut associated lymphoid tissue etc. In addition, functional aspects of the bowel such as peristalsis also play a role in resisting invasion of microbial products. All these factors are not measured with sugar probes. Moreover, the results of the sugar absorption tests are influenced by other factors such as hydration ${ }^{32}$. But more importantly, although we know that bacterial translocation across the gut occurs, its relation to septic morbidity in humans is disputed 33,34 . Despite these arguments we included the lactulose mannitol ratio as a primary outcome measure, because both parenteral nutrition and nutritional depletion have been correlated with functional deterioration of the gut barrier function as measured with these tests, and because currently there are no better non-invasive tests to assess intestinal permeability 35 . Our data showed no changes in the permeability of sugar probes within or between the groups. The extremely abnormal values in one cancer patient in the control group are unexplained, as this patient had no co-morbidity, medication, fluid infusion or symptoms that might explain this increased permeability. On average, the values found were in the same range as those found in a previous study 5 . The absence of an effect on the excretion ratio of lactulose and mannitol is in contrast to studies in animals as well as humans reporting an increased permeability after TPN6. Possibly the "damage" already occurs within the first 36 hours, and glutamine cannot repair the damaged barrier anymore. On the other hand, the values we found are on average not above normal values for a postabsorptive patient population 5 , rendering this assumption less likely. In the study by Buchman and coworkers, however, the conditions during measurement of intestinal permeability were different between baseline and the last measurement: whereas the first lactulose and mannitol solutions were administered after an overnight fast, the second determination of the lactulose/mannitol ratio took place during administration of total parenteral nutrition ${ }^{t}$. As referred to before, hydration alone can already account for changes in intestinal permeability as measured with sugar probes, thus making the value of this observation uncertain 32 .

In conclusion, this study demonstrates that in a nutritionally depleted patient population without major inflammatory stress, addition of glutamine to TPN does not result in improvements of intestinal permeability, mucosal morphology and gut mucosal inflammation. Systemic inflammatory reactions, which have profound effects on glutamine metabolism, are proposed to be of great importance in the final effect of glutamine on the gut. The effects of short term parenteral nutrition on intestinal morphology and function deserve further study. 


\section{ACKNOWLEDGEMENTS}

The authors acknowledge the help of Fresenius Kabi who provided the amino acid solutions and who supported the realization of this study. Mr. H. van Eijk and mr. D. Rooyakkers are acknowledged for their analytical help in the plasma amino acid concentrations. Furthermore we thank mrs. M. van de Wijngaarden for her help with the lactulose. mannitol and rhamnose determinations and the analists of the immunohistochemistry section of the department of Pathology for their help with the immunohistochemical stainings. Finally, we like to express our gratitude to all gastroenterologists and nurses of the endoscopy department for their help in this study. 


\section{REFERENCES}

1 Windmueller H G and Spaeth A E. Uptake and metabolism of plasma glutamine by the small intestine. The journal of biological chemistry. 1974; 249: 5070-5079

2 Wilmore D W, Smith R J, O'Dwyer S T, Jacobs D O, Ziegler T R and Wang X D. The gut: a central organ after surgical stress. Surgery. 1988; 104: 917.923

3 Souba W W. Intestinal glutamine metabolism and nutrition. J Nutr Biochem. 1993; 4: 2-9

4 Furst P, Albers $S$ and Stehle P. Glutamine-containing dipeptides in parenteral nutrition. $J$ Parenter Enteral Nutr. 1990; 14: 118S-124S

5 Hulst van der R R W J, van Kreel B K, Meyenfeldt von M F, Brummer R J, Arends J W, Deutz N E P and Soeters P B. Glutamine and the preservation of gut integrity. Lancet. 1993; 341: 1363-1365

6 Buchman A L, Moukarzel A A, Bhuta S, Belle M, Ament M E, Eckhert C D, Hollander D, Gombein J, Kopple J D and Vijayaroghavan S R. Parenteral nutrition is associated with intestinal morphologic and functional changes in humans. J Parenter Enteral Nutr. 1995; 19:453-460

7 Li J, Langkamp-Henken B, Suzuki K and Stahlgren L. Glutamine prevents parenteral nutrition-induced increases in intestinal permeability. J Parenter Enteral Nutr: 1994; 18: 303-307

8 Tremel H, Kienle B, Weiulemann LS, Stehle P and Furst P. Glutamine dipeptide - supplemented parenteral nutrition maintains intestinal function in the critically ill. Gastroenterology. 1994; 107: 1595-1601

9 De Blaauw I, Deutz N E P, van der Hulst R R W J and von Meyenfeldt M F. Glutamine depletion and increased gut permeability in nonanorectic, non-weight-losing tumor-bearing rats. Gastroenterology. 1997; 112: 118-126

10 Spaeth G, Gottwald T, Haas W and Holmer M. Glutamine peptide does not improve gut barrier function and mucosal immunity in total parenteral nutrition. I Parenter Enteral Nutr. 1993; 17: 317.323

11 Lipman T O. Bacterial translocation and enteral nutrition in humans: an outsider looks in. J Parenter Enteral Nutr: 1995; 19:156-165

12 Buchman A L. Glutamine: is it a conditionally required nutrient for the human gastrointestinal system? J Am Coll Nutr. 1996; 15: 199-205

13 Hulst van der R R W J, Deutz N E P, Meyenfeldt von M F; Elbers J M H, Stockbrügger R W and Soeters P B. Nutritional depletion and mucosal glutamine concentration. Clinical nutrition. 1994; 13:228-233

14 van der Hulst R R, von Meyenfeldt M F, van Kreel B K, Thunnissen F B, Brummer R J, Arends J W and Soeters P B. Cut permeability, intestinal morphology, and nutritional depletion. Nutrition. 1998; 14: 1-6

15 Soeters P B. Glutamine: the link between depletion and diminished gut function? J Am Coll Nutr. $1990 ; 15: 195.196$

10 Metropolitan Life Insurance Company. New weight standards for men and women. Bulletin Metropolitan Life Insurance Foundation. 1983; 64: 1.4

17 Roza A M and Shizgas H M. The Harris-Benedict equation reeavaluated: resting energy requirements and the body cell mass. Am J Clin Nutr. 1984; 40: 168-182

18 Ellis K J. Human body composition: in vivo methods. Physiological reviews. 2000; 80: 649-680

19 van Acker B A C, Hulsewé K W E, Wagenmakers A J M, von Meyenfeldt M F and Soeters P B. Response of glutamine metabolism to glutamine-supplemented parenteral nutrition. Am J Clin Nutr: 2000; 72: $790-795$

20 van Eijk $H$ M, van der Heijden $M A$, van Berlo $C L$ and Soeters P B. Fully automated liquidchromatographic determination of amino acids. Clin Chem. 1988; 34: 2510-2513 
21 Shippee R L, Johnson A A, Cioffi W G, Lasko J, LeVoyer T E and Jordan B S. Simultaneous determination of lactulose and mannitol in urine of burn patients by gas-liquid chromatography. Clin Chem. 1992 ; 38: $343-345$

22 Whitehead J. The design and analysis of sequential clinical trials, Ist edn. Chichester: Ellis Horwood, 1992

23 van der Hulst R R W J, von Meyenfeldt M F, Tiebosch A, Buurman W A and Soeters P B. Glutamine and intestinal immune cells in humans. J Parenter Enteral Nutr. 1997; $21: 310-315$.

24 Jackson N C, Carroll P V, Russell-Jones D L, Sonksen P H, Treacher D F and Umpleby A M. The metabolic consequences of critical illness: acute effects on glutamine and protein metabolism. $\mathrm{Am} /$ Physiol. 1900; 270: E103-170

25 Parry Billings $M$, Baigrie R J, Lamont P M, Morris P J and Newsholme E A. Effects of major and minor surgery on plasma glutamine and cytokine levels. Arch Surg. 1992; 127: 1237-1240

2 f. Powell Tuck J, Jamieson C P, Bettany G E, Obeid O, Fawcett H V, Archer C and Murphy D L. A double blind, randomised, controlled trial of glutamine supplementation in parenteral nutrition. Gut. 1999;
45: 82.88

27 Van Acker B A C, Hulsewé K W E, Wagenmakers A J M, Deutz N E P, Van Kreel B K, Halliday D, Matthews D E, Soeters $P B$ and Von Meyenfeldt M F. Absence of glutamine isotopic steady state: implications for the assessment of whole-body glutamine production rate. Clin Sci (ColCh). 1998; 95: $339-346$

28 Hulsewé K W E, van Acker B A C, Hameeteman W, von Meyenfeldt M F and Soeters P B. Effects of a bolus meal on intestinal mucosa in man. Clin Nutr. 1997; 10: 46

29 Unno $\mathrm{N}$ and Fink M P. Intestinal epithelial hyperpermeability. Mechanisms and relevance to disease Castroenterol Clin North Am. 1998; 27: 280-307

30 Buchman A L. Mestecky J, Moukarzel A and Ament M E. Intestinal immune function is unaffected by parenteral nutrition in man. J Am Coll Nutr. 1995; 14: 656-661

31 Uil J J, van Elburg R M, van Overbeek F M, Mulder C J, VanBerge-Henegouwen G P and Heymans $H$ S. Clinical implications of the sugar absorption test: intestinal permeability test to assess mucosal barrier function. Scand J Gastroenterol Suppl. 1997; 223: 70-78

32 Hallemeesch M M, Lamers W H, Soeters P B and Deutz N E P. Deutz N E P. Increased lactulose/rhamnose ratio Physiol. 2000; 278: G83.88

33 Lemaire L C, van Lanschot J J, Stoutenbeek C P, van Deventer S J, Wells C L and Gouma D J. Bacterial translocation in multiple organ failure: cause or epiphenomenon still unproven. Br J Surg. 1997; 84: $1340-1350$

34 MacFie J, O'Boyle C, Mitchell C J, Buckley P M, Johnstone D and Sudworth P. Gut origin of sepsis: a prospective study investigating associations between bacterial translocation, gastric microflora, and Septic morbidity. Gut. 1999; 45: 223-228

35 Bjarnason 1, MacPherson A and Hollander D. Intestinal permeability: an overview. Gastroenterology. 1905; 108: 1560.1581 


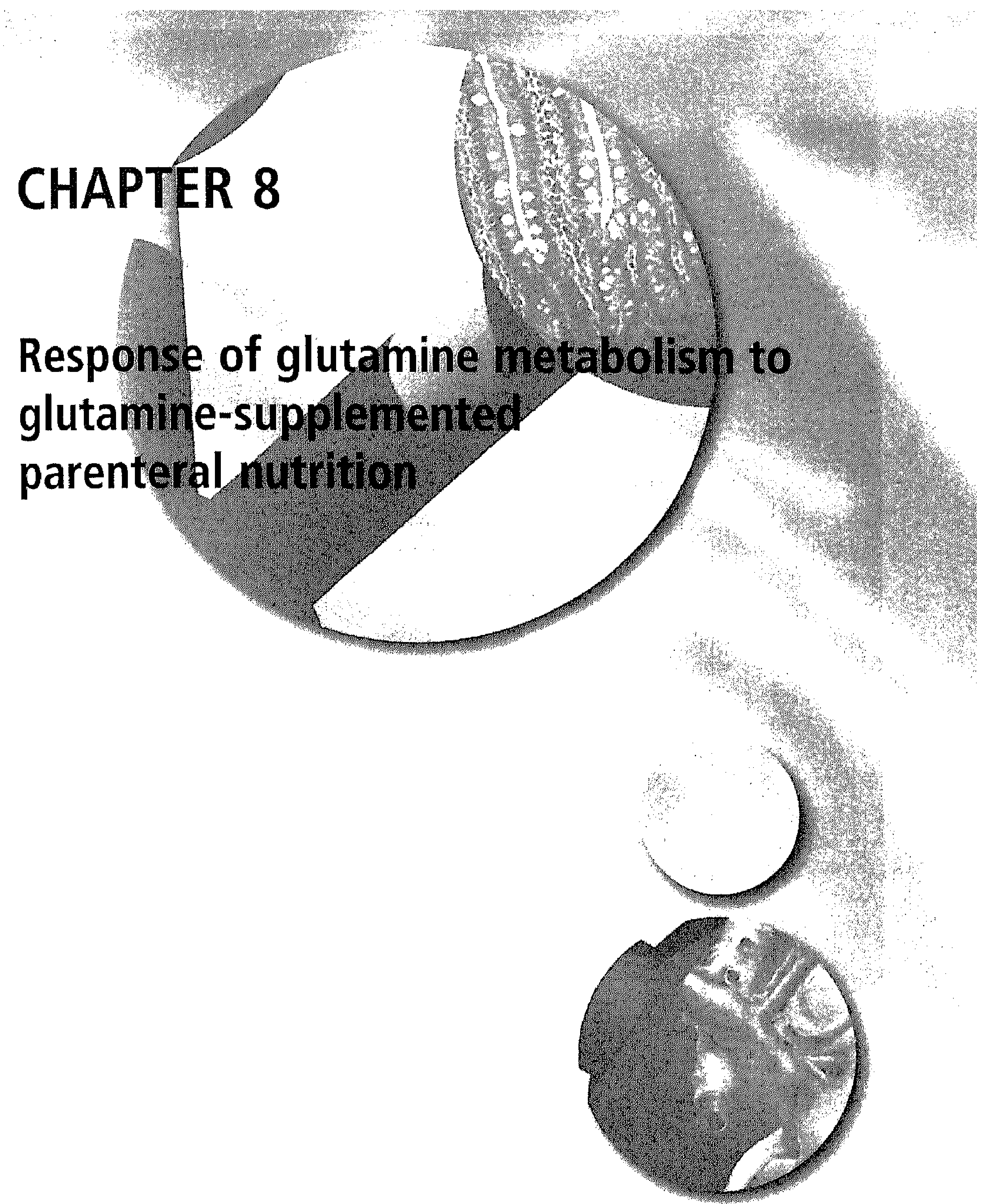

Bernadette AC van Acker; Karel WE Hulsewé, Anton JM Wagenmakers', Maarten F von Meyenfeldt, and Peter B Soeters

From the Department of Surgery, University Hospital Maastricht, and' the Department of Human Biology, Maastricht University, Maastricht, The Netherlands

Supported by the Dutch Cancer Foundation and by Pharmacia-Upjohn (Erlangen, Germany).

Published in Am J Clin Nutr 2000; 72:790-5. 


\section{Abstract}

\section{Background}

Increasing evidence suggests that glutamine is important for the function of many organ systems and supports the use of glutamine-enriched total parenteral nutrition (TPN) during severe illness. However, the effect of prolonged glutamine supplementation on glutamine kinetics has not been studied.

\section{Objective}

We investigated the effect of 8-10 days of TPN enriched with glutamine dipeptides on glutamine kinetics.

\section{Design}

Twenty-three preoperative patients were randomly allocated to receive either TPN enriched with glutamine dipeptides ( $60 \mu \mathrm{mol}$ glutamine $/ \mathrm{kg}$ body weight $\left.\mathrm{t}^{1} / \mathrm{h} \cdot 1\right)$ or isonitrogenous, isoenergetic, glutamine-free TPN. A primed, continuous, 6-h intravenous infusion of L-[5$\left.{ }^{15} \mathrm{~N}\right] \mathrm{glutamine}$ and $\mathrm{L}-\left[1 .{ }^{13} \mathrm{C}\right]$ leucine was given before (baseline) and 8-10 days after the TPN solutions were administered. Baseline measurements were performed after a 40-h administration of a standard solution of glucose and amino acids (no glutamine).

\section{Results}

Glutamine-enriched TPN increased the total appearance rate of glutamine $(\mathrm{P}<0.05)$ but did not inhibit or increase the endogenous appearance rate. The standard TPN solution also increased the glutamine appearance rate $(\mathrm{P}<0.05)$, but the change was much smaller than in the glutamine-supplemented group $(\mathrm{P}<0.01)$. The plasma glutamine concentration did not rise significantly during either treatment, suggesting increased tissue glutamine utilization, especially in the glutamine-supplemented group.

\section{Conclusion}

In view of the enhanced glutamine requirements in response to trauma and disease by tissues such as those of the gut, the immune system, and the liver, increased glutamine availability during glutamine-enriched TPN may be beneficial preoperatively in patients
with gastrointestinal disease. 


\section{INTRODUCTION}

Glutamine is an important nonessential amino acid and its intracellular concentration is much higher than that of other amino acids. Glutamine is released in large quantities from skeletal muscle and serves as an important carrier and donor of nitrogen!. Increasing evidence underscores the importance of glutamine for the function of many organ systems, including the intestine ${ }^{2}$ and the immune system ${ }^{3}$, and for maintaining the acid-base balance ${ }^{4}$.

The rationale for the inclusion of glutamine in dietary regimens stems from the hypothesis that glutamine becomes an essential nutrient during illness. Conventional amino acid solutions used for parenteral nutrition do not contain glutamine because glutamine is unstable in solution and is not soluble at high concentrations. The manufacture of synthetic dipeptides such as $L$. alanyl-L-glutamine and glycyl-L-glutamine, which are stable in solution and are rapidly hydrolyzed in plasma after intravenous infusion, has made it possible to administer total parenteral nutrition (TPN) solutions that contain glutamine. Clinical trials with glutamine-enriched TPN in different patient populations showed improved survival, a diminished incidence of clinical infections, improved nitrogen economy, and maintenance of intestinal integrity2,5.9.

Information regarding the response of glutamine kinetics to prolonged administration of glutamine-enriched TPN is not available. Therefore, it is not clear to what extent glutamine supplementation increases the amount of glutamine available to tissues and cells and to what extent it down-regulates endogenous glutamine production. The latter is observed after acute oral glutamine administration to healthy subjects 10 and to muscular dystrophy patients 11 and has been attributed to a decrease in estimates of both glutamine de novo synthesis and glutamine release from protein breakdown.

In this article we report the effect of the administration of glutamine dipeptide-enriched TPN for 8-10 days on the appearance rate of glutamine (Ra,gln) in plasma, on glutamine release from protein breakdown, and on de novo synthesis of glutamine in patients with gastrointestinal disease who were scheduled to receive TPN preoperatively.

\section{SUBJECTS AND METHOdS}

\section{Subjects}

Twenty-three patients admitted for gastrointestinal surgery and scheduled to receive TPN preoperatively were randomly allocated to receive either glutamine dipeptide-enriched TPN (GTPN) or standard (no glutamine) TPN (STPN). Patients were eligible for the study if they were nutritionally depleted. Nutritional depletion was defined as a body weight $<95 \%$ of ideal according to the Metropolitan Life Insurance Company ${ }^{12}$ or a loss in body weight of $>10 \%$ of the original weight within the previous 6 months. The patients were stratified according to neoplastic and nonneoplastic disease and were studied before surgery was performed. If there were any clinical signs of high metabolic stress, such as fever (> $\left.38.5^{\circ} \mathrm{C}\right)$, hypothermia $\left(<36^{\circ} \mathrm{C}\right)$, or evidence of sepsis or intraabdominal abscesses, the patients were excluded. Other exclusion criteria were renal or hepatic insufficiency, pregnancy, type 1 diabetes, ileus, heart failure, ascites, or receipt of parenteral nutrition within 14 days before the study. 
Patient characteristics are summarized in Table 1. Patients with Crohn's disease were hospitalized because of chronic stenosis $(n=4)$ and exacerbation $(n=1)$. One patient with chronic pancreatitis had pseudocysts; the 2 other patients had an exacerbation of chronic pancreatitis. Stenosis of the colon was due to previous diverticulitis in all 4 subjects. Of the patients with gastric or colorectal cancer, none had signs of disseminated disease at surgery. The study was approved by the ethics committee and review board of the University Hospital Maastricht and the procedures followed were in accordance with the Helsinki Declaration of 1964, as revised in 1989. Written, informed consent was obtained from all patients before commencement of the investigation. All investigators except the pharmacist were blinded to the randomization.

Table $1 \quad$ Patient characteristics at study entry 1

\begin{tabular}{|c|c|c|}
\hline & $\begin{array}{l}\text { Glutamine } \\
\text { supplemented TPN } \\
(n=6 \mathrm{M}, 4 \mathrm{~F})\end{array}$ & $\begin{array}{l}\text { Standard TPN } \\
(\mathrm{n}=9 \mathrm{M}, 4 \mathrm{~F})\end{array}$ \\
\hline $\operatorname{Age}(y)$ & $60 \pm 6^{2}$ & $57 \pm 4$ \\
\hline Weight $(\mathrm{kg})$ & $61 \pm 4$ & $63 \pm 2$ \\
\hline Height $(\mathrm{cm})$ & $171 \pm 4$ & $173 \pm 3$ \\
\hline Percentage ideal body weight (\%) & $93 \pm 4$ & $94 \pm 3$ \\
\hline Weight loss $(\%)^{3}$ & $17 \pm 3$ & $14 \pm 2$ \\
\hline Fat-free mass $(\mathrm{kg})$ & $49 \pm 4$ & $50 \pm 3$ \\
\hline Serum albumin $(g / L)$ & $33 \pm 3$ & $32 \pm 2$ \\
\hline Plasma glutamine ( $\mu \mathrm{mo} / \mathrm{L})$ & $578 \pm 38$ & $597 \pm 40$ \\
\hline \multicolumn{3}{|l|}{ Diagnosis $(n)$} \\
\hline Gastric cancer & 2 & 1 \\
\hline Colorectal cancer & 3 & 5 \\
\hline Benign stenosis of colon & 3 & 1 \\
\hline Crohn disease & 1 & 4 \\
\hline Pancreatitis & 1 & 2 \\
\hline
\end{tabular}

TThere were no significant differences between treatment groups (Fisher's exact test). TPN, total parenteral nutrition.

2 mean \pm SEM.

${ }^{3}$ Within the past 6 months.

\section{Feeding regimen}

TPN was administered via a central venous catheter. On the first 2 days, all patients received a commercially available standard solution of glucose and amino acids (Nutriflex; NPBI International BV, Emmer-Compascuum, Netherlands) in an amount of $50 \%$ of the planned study mixture volume. This baseline infusion period was intended to create a more standardized condition at baseline, to improve comparability of the patients at the onset of the study. After the 2-day baseline infusion, patients received the required amount of study 
GTPN or STPN for an additional 8-10 days (mean \pm SD: $9 \pm 1$ day). Fat, glucose, and amino acids were given in an "all-in-one" solution, formulated according to the measured body weight and the calculated total energy expenditure of the patient. The TPN solutions were prepared freshly each day under sterile conditions by the Department of Clinical Pharmacy (University Hospital Maastricht). The GTPN and STPN formulas were isonitrogenous $(0.24 \pm 0.01 \mathrm{~g} \mathrm{~N} / \mathrm{kg} /$ day) and isoenergetic (159 $\pm 5 \mathrm{~kJ} / \mathrm{kg} /$ day) and had equal volumes. The GTPN group received TPN supplemented with the dipeptides glycylLtyrosine and glycyl-L-glutamine. Patients received a total amount of $0.21 \pm 0.01 \mathrm{~g}$ glutamine $/ \mathrm{kg} /$ day. The GTPN solution had fewer total essential amino acids than did the STPN solution (Table 2). Nonprotein energy accounted for $134 \pm 4 \mathrm{~kJ} / \mathrm{kg} /$ day. Glucose and fat (Intralipid 20\%; Pharmacia-Upjohn, Erlangen, Germany) each contributed 50\% to the nonprotein energy content. Standard electrolytes, trace elements, and vitamins were added to the TPN solutions according to individual requirements and hospital routine. The daily doses of electrolytes and micronutrients are shown in Table 3. During the study period, a maximum of $500 \mathrm{~mL}$ water or tea per day was allowed orally.

Table 2 Compositions of the total-parenteral-nutrition (TPN) solutions in $g / L^{I}$

\begin{tabular}{lcc}
\hline & Glutamine-supplemented TPN & Standard TPN \\
\hline Fat & 48 & 48 \\
Glucose & 120 & 120 \\
Total amino acids & 40.3 & 39.7 \\
Isoleucine & 1.6 & 2.0 \\
Leucine & 2.3 & 2.8 \\
Lysine & 2.6 & 3.2 \\
Methionine & 1.6 & 2.0 \\
Phenilalanine & 1.7 & 2.8 \\
Tyrosine & 0.7 & 0.1 \\
Threonine & 1.6 & 2.0 \\
Tryptophan & 0.5 & 0.7 \\
Valine & 2.1 & 2.6 \\
Alanine & 4.7 & 5.7 \\
Arginine & 3.3 & 4.0 \\
Asparagine & 1.0 & 1.2 \\
Glutamate & 1.6 & 2.0 \\
Histidine & 2.0 & 2.4 \\
Proline & 2.0 & 2.4 \\
Serine & 1.3 & 1.6 \\
Glycine & 3.3 & 2.8 \\
Glutamine & 5.8 & 0.0 \\
\hline
\end{tabular}

1The volume of TPN administered each day averaged $2.2 \pm 0.3 \mathrm{~L}$ (mean \pm SD). 
Table 3 Mean doses of electrolytes, trace elements, and vitamins administered via the total-parenteral. nutrition (TPN) solutions ${ }^{l}$

\begin{tabular}{|c|c|c|}
\hline Nutrient & Glutamine-supplemented TPN & Standard TPN \\
\hline Sodium (mmol/d) & 58 & 46 \\
\hline Potassium (mmol/d) & 80 & 80 \\
\hline Magnesium (mmol/d) & 6 & 6 \\
\hline Calcium (mmol/d) & 5 & 5 \\
\hline Chloride (mmol/d) & 62 & 56 \\
\hline Acetate $(\mathrm{mmo} / / \mathrm{d})$ & 69 & 63 \\
\hline Phosphate (mmol/d) & 23 & 22 \\
\hline Zinc (mg/d) & 6.5 & 6.5 \\
\hline Copper (mg/d) & 1 & 1 \\
\hline Manganese $(\mathrm{mg} / \mathrm{d})$ & 0.3 & 0.3 \\
\hline lodine $(\mu g / d)$ & 120 & 120 \\
\hline Chromium ( $\mu g / d)$ & 10 & 10 \\
\hline Selenium ( $\mu g / d)$ & 60 & 60 \\
\hline Thiamine (mg/d) & 3.5 & 3.5 \\
\hline Riboflavin (mg/d) & 4.2 & 4.2 \\
\hline Pyridoxine (mg/d) & 4.2 & 4.2 \\
\hline Nicotinamide (mg/d) & 28 & 28 \\
\hline Biotin (mg/d) & 0.7 & 0.7 \\
\hline Dexpantenol $(\mathrm{mg} / \mathrm{d})$ & 28 & 28 \\
\hline Vitamin C (mg/d) & 100 & 100 \\
\hline
\end{tabular}

1There were no significant differences between the 2 solutions.

\section{Study design}

On the morning of day 2, i.e. 1.5 days after administration of the standard glucose and amino acid solution began, 2 catheters were placed in the subjects: 1 in an antecubital vein for isotope infusion and 1 in the radial artery for blood sampling. The arterial catheter was kept patent by a slow saline infusion. At 08.30 , a primed, constant intravenous infusion of $\mathrm{L}-[5.15 \mathrm{~N}]$ glutamine $(0.68 \mu \mathrm{mol} / \mathrm{kg} / \mathrm{h}$; prime $0.68 \mu \mathrm{mol} / \mathrm{kg})$ and $\mathrm{L}-[1.13 \mathrm{C}]$ leucine $(7.63$ $\mu \mathrm{mol} / \mathrm{kg} / \mathrm{h}$; prime $7.63 \mu \mathrm{mol} / \mathrm{kg}$ ) began and continued for 6 hours. The tracers were purchased from Cambridge Isotope Laboratories (Woburn, MA). Sterile and pyrogen-free stock solutions of the tracers had been prepared by the Department of Clinical Pharmacy and were kept at room temperature $(\mathrm{L}-[1-13 \mathrm{C}]$ leucine $)$ or $-80^{\circ} \mathrm{C}(\mathrm{L}-[5-15 \mathrm{~N}] \mathrm{glutamine})$. The stock solutions were diluted with normal saline before the start of each tracer infusion. At the end of the tracer infusion, a sample of the infusate was taken for measurement of the concentration and enrichment of leucine and glutamine.

Blood samples were drawn into chilled heparin-containing tubes before the start of the tracer infusion for measurement of background enrichment and 2,3,4,5, and 6 hours after onset of the infusion. Plasma was obtained by centrifugation of whole blood at $2200 \mathrm{~g}$ at 
$4^{\circ} \mathrm{C}$ for 5 minutes. For the measurement of plasma glutamine concentration, plasma was deproteinized with sulfosalicylic acid, vortex mixed, frozen in liquid nitrogen, and stored at $-80^{\circ} \mathrm{C}$. For tracer enrichment measurements, plasma was frozen and stored at $-80^{\circ} \mathrm{C}$, until analyzed. Tracer infusion and blood sampling were repeated on the last day of GTPN and STPN treatment to investigate the effect of these TPN solutions on glutamine and protein metabolism. So that conditions were standard, the tracers were infused from 08.30 to 14.30 on both days while the patients were in a semirecumbent position in bed, and the catheters were inserted in the same position.

\section{Analytic methods}

Plasma enrichment of glutamine [mole percent excess (MPE)] was measured by using a tertbutyldimethylsilyl derivative and gas chromatography-combustion isotope ratio mass spectrometry (MAT 252; Finnigan, Bremen, Germany), as described previously ${ }^{3}$. Plasma enrichment of $\alpha$-ketoisocaproate $\left(E_{\mathrm{p}, \mathrm{KI}}\right.$; in MPE) was measured by using a quinoxalinol. trimethylsilyl derivative and gas chromatography-mass spectrometry (Finnigan Incos $\mathrm{XL}$; San Jose, CA) in a manner similar to that described previously ${ }^{14}$. Final values for KIC determinations were corrected by using calibration curves. The concentration of glutamine in plasma and in the tracer infusate was determined by fully automated HPLC ${ }^{15}$.

\section{Calculations}

The $\mathrm{R}_{\mathrm{a}, \mathrm{gln}}$ in plasma (in $\mu \mathrm{mol} / \mathrm{kg}^{-1} / \mathrm{h}^{-1}$ ) was calculated as

$$
R_{a, g l n}=i_{[15 N|g| n}\left[\left(E_{i, g \mid n} / E_{p, g l n}\right)-1\right]
$$

where $i_{115 N \mid g l n}$ is the tracer infusion rate (in $\mu \mathrm{mol} / \mathrm{kg} / \mathrm{h}$ ) and $E_{1, g \mathrm{gn}}$ is the enrichment of glutamine (in MPE) in the tracer infusate, and $E_{p, g l n}$ is the mean plasma glutamine enrichment between 2 and 6 hours of tracer infusion. As shown previously, when $R_{a, g l n}$ is obtained in this way it is overestimated by $\geq 20 \%$ because of slow equilibration of the glutamine tracer in the large muscle glutamine pool ${ }^{13}$.

During GTPN administration, endogenous $R_{a, g h n}\left(\right.$ Endo $\left._{a, g l n}\right)$ in plasma was calculated as

$$
\text { EndoR }_{\mathrm{a}, \mathrm{gln}}=\mathrm{R}_{\mathrm{a}, \mathrm{gln}}-\operatorname{Inf}_{\mathrm{gln}}
$$

where $\operatorname{Inf}_{\text {gln }}$ is the rate of intravenous delivery of glutamine (exogenous glutamine) from TPN. When no exogenous glutamine is delivered:

$$
\text { Endo } R_{a, g l n}=R_{a, g l n}
$$

In the absence of exogenous glutamine, 2 sources contribute to the $\mathrm{R}_{\mathrm{a}, \mathrm{gln}}$ : proteolysis and de novo synthesis. Theoretically, shrinkage of the intracellular free glutamine pool may also contribute to the $R_{a, g l n}$, but we assumed that the amount of glutamine release from this source was negligible during the study period. Proteolysis was measured by using the endogenous whole-body appearance rate of leucine $\left(E\right.$ do $R_{a, \text { leu; }}$ in $\left.\mu \mathrm{mol} / \mathrm{kg} / \mathrm{h}\right) . \mathrm{R}_{\mathrm{a}, \text { leu }}$ was calculated by using plasma KIC enrichment ${ }^{16}$ : 


$$
R_{a, \text { leu }}=i_{[13 C \| \text { eu }} X\left\lfloor\left(E_{i, \text { leu }} / E_{p, K I C}\right) \cdot 1\right]
$$

Where $\dot{i}_{13 C l \mid e u}$ is the tracer infusion rate in $\mu \mathrm{mol} / \mathrm{kg} / \mathrm{h}, \mathrm{E}_{\mathrm{i}, \mathrm{leu}}$ is the enrichment of leucine (in MPE) in the tracer infusate, and $E_{p, K I C}$ is the average enrichment of KIC in plasma between 2 and 6 hour of tracer infusion. Endo $R_{a, l e u}$ was calculated as

$$
\text { EndoR } \mathrm{R}_{\mathrm{a}, \text { leu }}=\mathrm{R}_{\mathrm{a}, \text { leu }}-\operatorname{Inf}_{\text {leu }}
$$

where $\operatorname{Inf}_{\text {leu }}$ is the rate of TPN-derived exogenous leucine infusion from $R_{a, \text { leu. }}$. Glutamine arising from protein degradation $\left(\mathrm{PD}_{\mathrm{gln}}\right)$ was calculated as

$$
\mathrm{PD}_{\text {gin }}=\mathrm{R}_{\mathrm{a}, \text { leu }} \times(4.32 \times 131) /(9.18 \times 146)
$$

where 4.32 and 9.18 are the assumed glutamine and leucine contents of body protein (g/100 g protein), respectively, and 146 and 131 are glutamine and leucine molecular weights $(\mathrm{g} / \mathrm{mol})$, respectively ${ }^{17}$. Glutamine arising from de novo synthesis $\left(\mathrm{DNS}_{\mathrm{gln}}\right)$ was
calculated as

$$
\mathrm{DNS}_{\mathrm{gln}}=\text { EndoR }_{\mathrm{a}, \mathrm{gln}}-\mathrm{PD}_{\mathrm{gln}}
$$

\section{Statistics}

Data are presented as means \pm SEMs, unless stated otherwise. The Mann-Whitney U test was used to compare the treatment groups at baseline. Within each group, the effect of 8-10 days of TPN was tested by using the Wilcoxon signed-rank test. Fisher's exact test was performed to determine whether the number of patients with pancreatitis and Crohn's disease was different between the groups. A two-factor repeated-measures analysis of factor, was performed to within-subjects factor and treatment as the between-subjects by-treatment interaction $A$ P Pine the main effects of time and treatment and the timeanalyses were performed A P value $<0.05$ was considered statistically significant. The

\section{RESULTS}

On admission there were no significant differences in patient characteristics, nutritional status, or underlying disease between the GTPN and STPN groups (Table 1). The number of patients with pancreatitis and Crohn's disease was not significantly different between the groups. During the 10-day study period, 2 patients in the GTPN group required surgical intervention for reasons unrelated to the TPN (tumor bleeding and intraabdominal abscess drainage), 1 patient with colorectal cancer in the STPN group refused tracer infusion and blood sampling at the end of study, and 1 patient with stenosis of the colon in the STPN group discontinued TPN because of an unexplained generalized rash. One patient with excluded because portal hTPN group was suspected to have portal hypertension and was excluded because portal hypertension is likely to influence glutamine metabolism. 
In the remaining 18 patients, there were no significant differences in patient characteristics or in the type of underlying disease between the treatment groups. The volume of feeding per day was similar in both groups: $2219 \pm 135$ and $2243 \pm 78 \mathrm{~mL} /$ day in the GTPN and STPN groups, respectively. Addition of $0.21 \pm 0.01 \mathrm{~g}$ glutamine $/ \mathrm{kg}$ body weight $1 / \mathrm{day}{ }^{1}$ for $9 \pm 1$ days did not induce a rise in the concentration of plasma glutamine. On day 2 , when $50 \%$ of energy requirements was provided by a glucose and amino acid infusion, the plasma glutamine concentration in the STPN group was $564 \pm 27 \mu \mathrm{mol} / \mathrm{L}$; the concentration was $592 \pm 38 \mu \mathrm{mol} / \mathrm{L}$ at the end of study during full feeding. In the GTPN group, plasma glutamine concentrations were $610 \pm 39 \mu \mathrm{mol} / \mathrm{L}$ at study entry and $641 \pm 42 \mu \mathrm{mol} / \mathrm{L}$ after 8-10 days of GTPN infusion.

The time courses of plasma $\left[{ }^{15} \mathrm{~N}\right]$ glutamine and $[1-13 \mathrm{C}] \mathrm{KIC}$ enrichment are shown in Figure 1. Similar KIC enrichment values were obtained before and after treatment. Glutamine enrichment in plasma decreased in both treatment groups but to a larger extent in the GTPN group ( $\mathrm{P}<0.05)$. At baseline, there were no significant differences in $R_{\mathrm{a}, \text { gin }}$, PDgln, DNSgIn or $\mathrm{R}_{\mathrm{a} \text {, leu }}$ between the 2 groups. Whole-body protein degradation during treatment, estimated from Endo $\mathrm{R}_{\mathrm{a}, \text { leu}}$, did not change significantly from baseline in either group: from $81 \pm 4$ to $79 \pm 5 \mu \mathrm{mol} / \mathrm{kg} / \mathrm{h}$ in the STPN group and from $96 \pm 7$ to $96 \pm 11$ $\mu \mathrm{mol} / \mathrm{kg} / \mathrm{h}$ in the GTPN group.
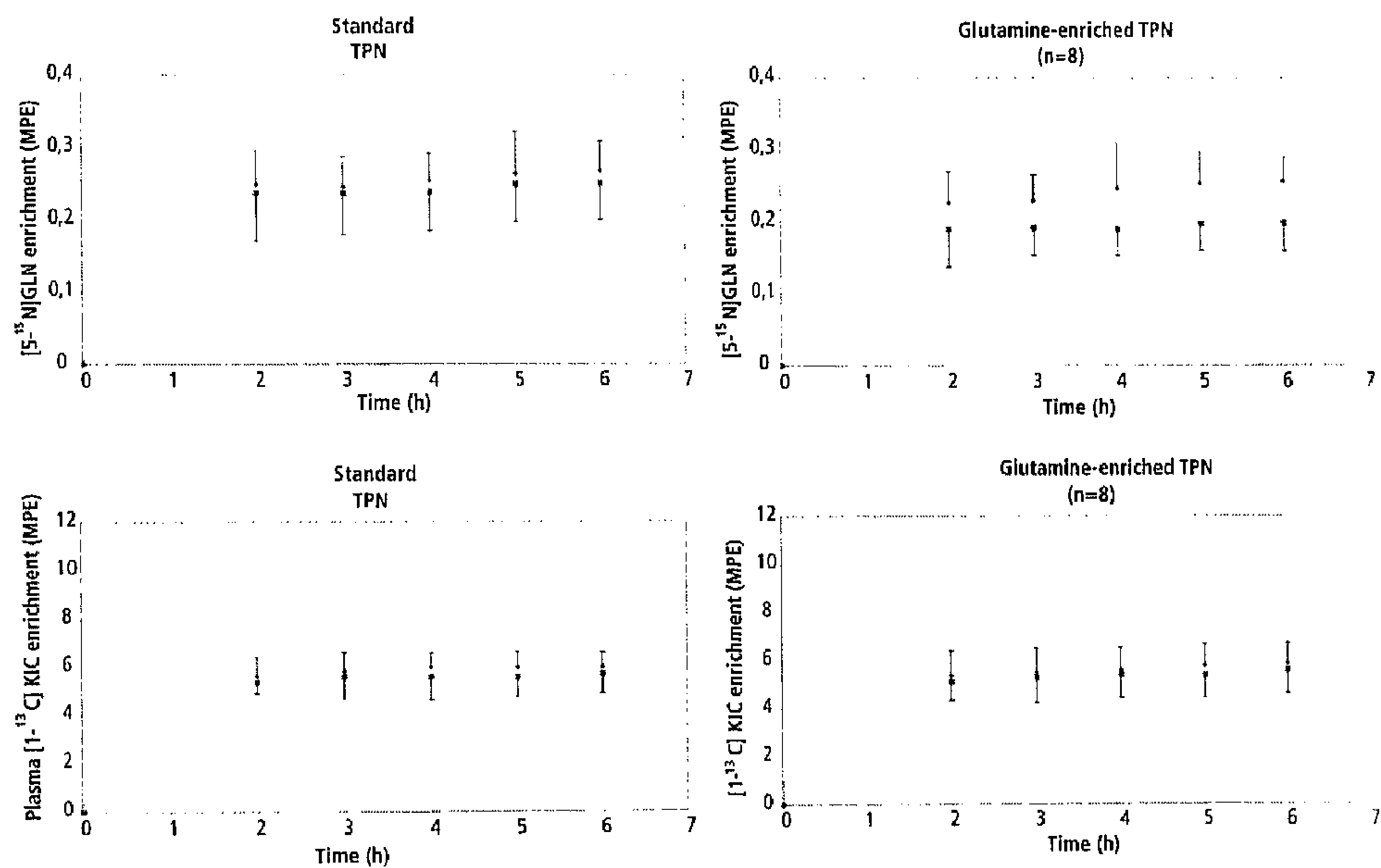

Figure 1 Mean ( \pm SD) plasma glutamine (Gln) and ketoisocaproate (KIC) enrichment at baseline / and after $9 \pm 1$ day (O) of standard or glutamine-enriched total parenteral nutrition (TPN). Statistical analysis was performed by using two-factor repeated-measures analysis of variance using the average values between 2 and 6 h of tracer infusion. Gin enrichment showed a significant effect of time (baseline versus 9 days after treatment: $P<0.001$ ) and a significam time-by-treatment interaction $(P<0.005)$ MPE, mole percent excess. 
The response of glutamine kinetics to GTPN or STPN is shown in Figure 2. Although the total $R_{a, g n}$ in plasma increased significantly in both treatment groups (from $281 \pm 16$ to 361 $\pm 34 \mu \mathrm{mol} / \mathrm{kg} / \mathrm{h}$ in the GTPN group and from $250 \pm 8$ to $267 \pm 14 \mu \mathrm{mol} / \mathrm{kg} / \mathrm{h}$ in the STPN group), the rise in $R_{a, \text { gln }}$ observed during GTPN treatment was significantly larger than that observed during STPN treatment $(\mathrm{P}<0.01)$. As shown in Figure 2, the rise in $R_{a, g l n}$ observed in the GTPN group, was largely attributed to parenteral glutamine administration. Whereas Endo $\mathrm{R}_{\mathrm{a}, \mathrm{gln}}$ increased significantly during STPN treatment (from $250 \pm 8$ to $267 \pm 14 \mu \mathrm{mol} / \mathrm{kg} / \mathrm{h}$ ), no significant change occurred in the Endo $\mathrm{R}_{\mathrm{a}, \mathrm{gin}}$ of GTPN-treated patients (from $281 \pm 16$ to $299 \pm 31 \mu \mathrm{mol} / \mathrm{kg} / \mathrm{h}$ ). Administration of GTPN did not change $\mathrm{PD}_{\mathrm{gln}}$ (from $40 \pm 3$ to $41 \pm 5 \mu \mathrm{mol} / \mathrm{kg} / \mathrm{h}$ ) or $\mathrm{DNS}_{\mathrm{gln}}$ (from $241 \pm 14$ to 258 $\pm 27 \mu \mathrm{mol} / \mathrm{kg} / \mathrm{h}$ ) significantly. In the STPN group, $\mathrm{PD}_{\text {gln }}$ did not change significantly (34 $\pm 2 \mu \mathrm{mol} / \mathrm{kg} / \mathrm{h}$ at baseline and $33 \pm 2 \mu \mathrm{mol} / \mathrm{kg} / \mathrm{h}$ at the end of the study), whereas $D N S_{\mathrm{gln}}$ increased significantly from $216 \pm 8$ to $234 \pm 13 \mu \mathrm{mol} / \mathrm{kg} / \mathrm{h}$.

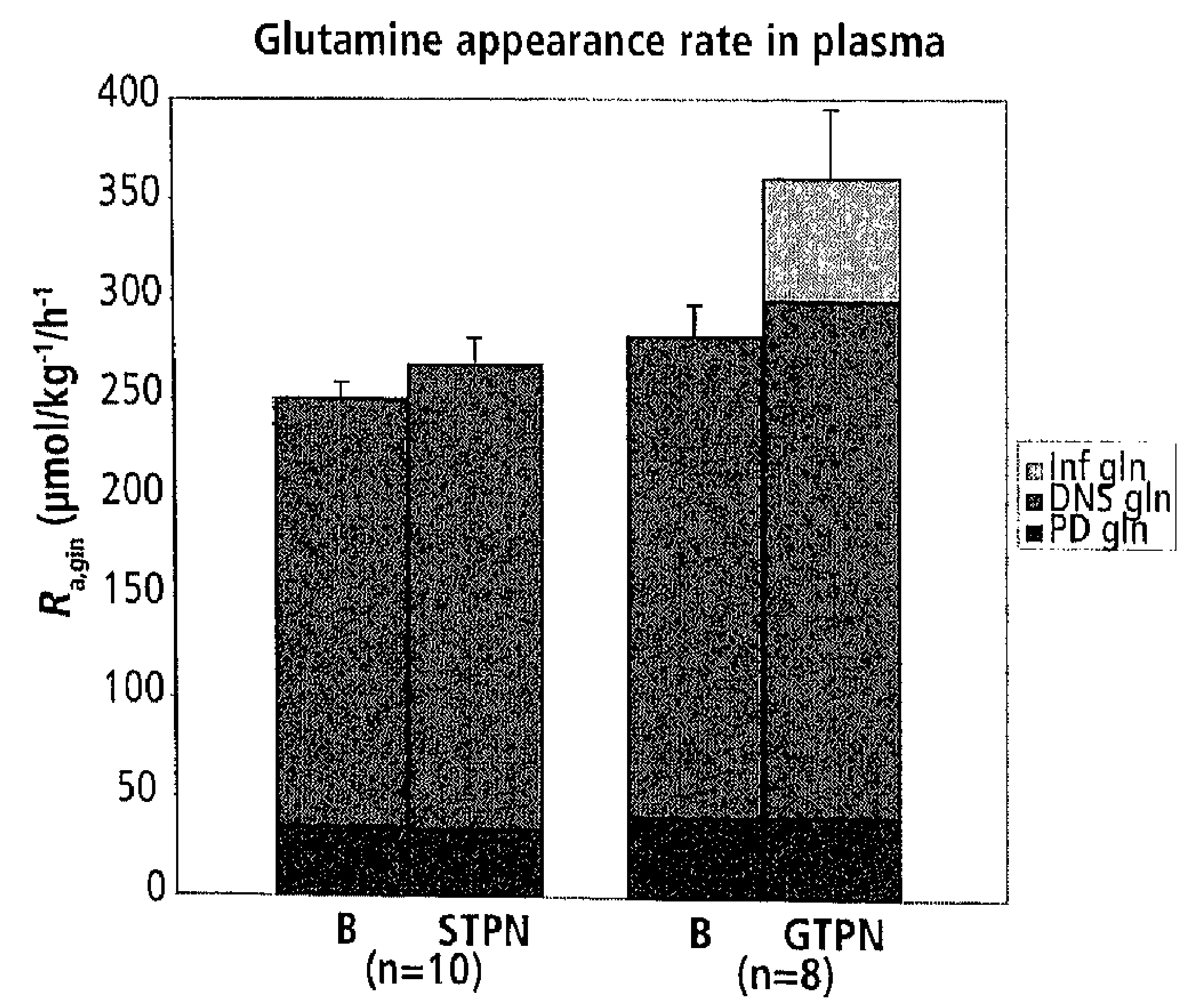

Figure 2 Mean $( \pm S E M)$ glutamine appearance rate (Ra) in plasma at baseline (B) and during either standard total parenteral nutrition (STPN) or glutamine (GIn)-enriched total parenteral nutrition (CTPN). Statistical analysis was performed by using two-factor repeated-measures analysis of varlance. Significant effects of time (baseline versus 9 days after treatment) were shown for $R_{a, g l n}(P<0.001)$ and for $D N S_{\text {gln }}(P<0.05)$. A significant effect of treatment $(G T P N$ versus STPN; $P<0.05)$ and a significant time-by-treatment interaction $(P<0.005)$ were shown for $R_{a, g l n^{\prime}} P D_{g l n}$ glutamine arising from protein degradation; $D N S_{g ! n}$ glutamine arising from de novo synthesis; Inf gir exogenous glutamine. 


\section{Discussion}

In this study, $\mathrm{R}_{\mathrm{a}, \mathrm{gln}}$, as measured from the dilution of infused L-[5.15N|glutamine in plasma, increased to a larger extent after 8-10 days of GTPN than after an equal period of STPN. Endo $\mathrm{R}_{\mathrm{a}, \mathrm{gm} \text { (ie }}$ (ie, $\mathrm{PD}_{\mathrm{gin}}$ and $\mathrm{DNS}_{\mathrm{gln}}$ ) was not affected adversely by the inclusion of glutamine in the TPN solution.

To our knowledge, the present study was the first to evaluate the effect of prolonged parenterally administered glutamine on glutamine kinetics. Acute oral glutamine administration in a much larger dose than that used in our study $(800 \mu \mathrm{mol} / \mathrm{kg} / \mathrm{h}$ for $5 \mathrm{~h})$ resulted in a $20-30 \%$ decrease in Endo $R_{a, g l n}$, both in healthy volunteers ${ }^{10}$ and in children with Duchenne muscular dystrophy". The decrease in the estimated $\mathrm{DNS}_{\mathrm{gln}}$ accounted for $90 \%$ of the decrease in Endo $R_{a, g n}$. $P D_{\text {gln }}$ also decreased in the 2 studies, but to a smaller extent and not significantly so in the healthy volunteers 10 . On the basis of these findings, the authors suggested that acute oral glutamine administration might have an acute protein-sparing effect by decreasing wholebody protein degradation and $\mathrm{DNS}_{\mathrm{gln}}$, therefore sparing nitrogen precursors 1 .

The route and quantity of glutamine administration were not the only aspects in which the present study differed from the previous investigations. Hankard et al 10,11 studied subjects postabsorptively during an intervention with glutamine supplementation compared with saline or flavored water, whereas in the current study patients were in the fed state and received either glutamine-supplemented TPN or an isonitrogenous solution of glutamine-free TPN. The 2-day baseline TPN infusion used in the present study may have influenced the results. Ingestion of protein and amino acids was shown previously to decrease protein breakdown and increase $\mathrm{DNS}_{\mathrm{gn}}$ in muscle ${ }^{1,18}$. The latter is attributed to increased availability of the precursors for glutamine production in muscle: glutamate, aspartate, asparagines, and the branched-chain amino acids ${ }^{1}, 18$. Therefore, the present finding that protein degradation was not affected by 9 days of GTPN or STPN infusion may have resulted from the fact that the patients had already received TPN at entry. Compared with the baseline measurements, when $50 \%$ of energy requirements was provided by an infusion of glucose and amino acids, administration of the full amount of TPN did not further decrease protein breakdown in the present study. Additionally, replacement of part of the amino acids in the TPN solution with glutamine had no additional effect on protein degradation.

A small increase from baseline in $\mathrm{DNS}_{\mathrm{gln}}$ occurred during infusion of both GTPN and STPN, which was significant in the STPN group but not in the GTPN group. Differences in the administered amount of glutamine precursors probably underlie this gradual response in $\mathrm{DNS}_{\text {gin }}$ because the sum of glutamate, aspartate, asparagines, and branched-chain amino acids was $11 \%$ higher during GTPN than at baseline and as much as $36 \%$ higher during STPN than at baseline. Therefore, the 2-day baseline TPN infusion may also explain the lack of a significant rise in $\mathrm{DNS}_{\mathrm{gn}}$ in the GTPN group.

Whereas Endo $R_{a, g l n}$ did not change significantly in the GTPN group, systemic $R_{a, g i n}$ increased significantly, indicating that more glutamine was available for uptake by cells that need this amino acid. In view of the important role of glutamine in many metabolic processes leg, a vehicle for interorgan transport of nitrogen and carbon skeletons 1,19 , a metabolic substrate for rapidly dividing cells20,21, a precursor for nucleotides and 
glutathion $e^{22,23}$, and a regulator of acid-base balance 4], an increased amount of available glutamine is supposedly beneficial during periods of inadequate endogenous glutamine production resulting from glutamine depletion, increased glutamine requirements, or both. Such periods may occur during severe illness, when the need for glutamine is enhanced, as indicated by the increased uptake of glutamine by the liver and the spleen after trauma 24 . Prolonged periods of high metabolic stress eventually lead to depletion of endogenous glutamine stores. Consequently, the body may no longer have the capacity to cope with the increased requirements.

The increase in total $R_{a \text { agn }}$ observed during GTPN was in proportion to the amount of glutamine in the supplemented dipeptides, suggesting rapid hydrolysis in plasma and complete bioavailability of glutamine in the synthetic dipeptide. Thus, the lack of a significant rise in plasma glutamine concentrations in the GTPN group was not due to incomplete bioavailability of the glutamine dipeptide, but rather to increased utilization of glutamine. A previous study by our group showed that parenteral glutamine administration can increase plasma glutamine concentrations in nutritionally depleted patients in whom plasma glutamine concentrations are already low before TPN therapy is started 25 . Plasma glutamine concentrations at baseline were in the normal range in the present group of patients. Apparently, the quantity of intravenously infused glutamine in this study, which amounted to $20-25 \%$ of EndoR $\mathrm{R}_{\mathrm{a}, \mathrm{gln}}$, is instantaneously extracted from the plasma pool. Much larger quantities of glutamine probably need to be administered to induce increased plasma concentrations in subjects with initial concentrations in the normal range $e^{9 \cdot 11}$.

In conclusion, we showed that administration of glutamine dipeptide-supplemented TPN to nutritionally depleted patients increases $R_{a}$,gln in plasma without inhibiting endogenous glutamine production. The lack of a significant rise in plasma glutamine concentrations with glutamine infusion suggests that tissue glutamine utilization increased. In view of the enhanced glutamine requirements in response to trauma and disease by tissues such as those of the gut, the immune system, and the liver, increased glutamine availability during glutamine-enriched TPN may be beneficial for the type of patients included in this study.

We acknowledge M Meers, AP Gijsen, and F van de Vegt for measuring glutamine and KIC enrichments; $H$ van Eijk for measuring amino acid concentrations; $E$ Hardy (Department of Clinical Pharmacy) for handling the randomization procedure and for preparing the TPN solutions; and M Rouflart for practical advice and assistance in the management of the patients. 


\section{REFERENCES}

1. Elia M, Folmer P, Schlatmann A, Goren A, Austin S. Amino acid metabolism in muscle and in the whole body before and after ingestion of a single mixed meal. Am J Clin Nutr. 1989; 49:1203-1210.

2. van der Hulst RRWJ, van Kreel BK, Meyenfeldt von MF, et al. Glutamine and the preservation of gut integrity. Lancet. 1993; 341:1363-1365.

3. Calder PC. Glutamine and the immune system. Clin Nutr. 1994; 13:2.

4. Halperin ML, Ethier JH, Kamel KS. The excretion of ammonium ions and acid base balance. Clin Biochem. 1990; 23:185-188.

5. Griffiths RD, Jones C, Palmer TF. Six-month outcome of critically ill patients given glutamine. supplemented parenteral nutrition. Nutrition. 1997; 13:295.302.

6. Lacey JM, Crouch JB, Benfell $\mathrm{K}$, et al. The effects of glutamine-supplemented parenteral nutrition in premature infants. JPEN J Parenter Enteral Nutr: 1996; 20:74-80.

7. Morlion BJ, Stehle P, Wachtler P, et al. Total parenteral nutrition with glutamine dipeptide after major abdominal surgery: a randomized, double-blind, controlled study. Ann Surg. 1998; 227:302-308.

8. Schloerb PR, Amare M. Total parenteral nutrition with glutamine in bone marrow transplantation and other clinical applications (a randomized, double-blind study). J Parenter Enteral Nutr. 1993; 17:407. 413.

9. Ziegler TR, Young LS, Benfell $\mathrm{K}$, et al. Clinical and metabolic efficacy of glutamine-supplemented parenteral nutrition after bone marrow transplantation. A randomized, double-blind, controlled study. Ann Intern Med. 1992; 116:821-828.

10. Hankard RG, Darmaun D, Sager BK, D'Amore D, Parsons WR, Haymond M. Response of glutamine metabolism to exogenous glutanine in humans. Am J Physiol. 1995; 269:E603-E670.

11. Hankard RG, Hammond D, Haymond MW, Darmaun D. Oral glutamine slows down whole body protein breakdown in Duchenne muscular dystrophy. Pediatr Res. 1998; 43:222-220.

12. Metropolitan Life Insurance Company. New weight standards for men and women. Bulletin Metropolitan Life Insurance Foundation. 1983; 64:1-4.

13. Van Acker BAC, Hulsewé KWE, Wagenmakers AIM, et al. Absence of glutamine isotopic steady state: implications for the assessment of whole-body glutamine production rate. Clin Sci (Colch). 1998: 95:339-346.

14. Ford GC, Cheng KN, Halliday D. Analysis of $(1 \cdot 13 \mathrm{C})$ leucine and $(13 \mathrm{C}) \mathrm{KIC}$ in plasma by capillary gas chromatography/mass spectrometry in protein turnover studies. Biomed Mass Spectrom. 1985; $12: 432-436$

15. van Eijk HM, Rooyakkers DR, Deutz NE. Rapid routine determination of amino acids in plasma by high-performance liquid chromatography with a 2-3 microns Spherisorb ODS II column. J Chromatog: $1993 ; 620: 143 \cdot 148$.

16. Horber FF, Horber-Feyder CM, Krayer S, Schwenk WF, Haymond MW. Plasma reciprocal pool specific activity predicts that of intracellular free leucine for protein synthesis. Am J Physiol. 1989; 257:E385-399.

17. Kuhn KS, Schuhmann K, Stehle P, Darmaun D, Furst P. Determination of glutamine in muscle protein facilitates accurate assessment of proteolysis and de novo synthesis-derived endogenous glutamine production. Am J Clin Nutr. 1999; 70:484-489.

18. Elia $M$, Livesey $G$. Effects of ingested steak and infused leucine on forelimb metabolism in man and the fate of the carbon skeletons and amino groups of branched-chain amino acids. Clin Sci /Londi. $1983 ; 64: 517-526$. 
19. Nurihan N, Bucd A, Periello $G$, et al. Glutamine: a major gluconeogenic precursor and vehicle for interorgan carbon transport in man. J Clin Invest. 1995; 95:272-277.

20. Ardawi MS, Newsholme EA. Glutamine metabolism in lymphocytes of the rat. Biochem J. 1983; $212: 835.842$.

21. Windmueller HC. Glutamine utilization by the small intestine. Adv Enzymol. 1982:201-237.

22. Cao Y, Feng Z, Hoos A, Klimberg VS. Glutamine enhances gut glutathione production. J PEN J Parenter Enteral Nutr. 1908; 22:224.227.

23. Lacey MM, Wilmore DW. Is glutamine a conditionally essential amino acid? Nutr Rev. 1990; 48:297. 300.

24. Deutz NEP. Reijven PL, Athanasas $G$, Soeters PB. Post-operative changes in hepatic, intestinal, splenic and muscle fluxes of amino acids and ammonia in pigs. Clin Sci Colch. 1992; 83:607-014.

25. Hulst van der RRWJ, Meyenfeldt von MF, Deutz NEP, Stockbrügger RW, Soeters PB. The effect of glutamine administration on intestinal glutamine content, J Surg Res. 1996; 01:30-34. 


\section{CHAPTER 9}

Effects of Glutamine

enriched total parental Nutrition

on interorgan Amino Acid and

Gut Mucosal Glutathione Metabolism 


\section{INTRODUCTION}

Glutamine and glutamate exert a central role in cell metabolism ${ }^{1}$. In contrast to glutamate, glutamine plays important roles in interorgan transport as a non toxic nitrogen and carbon carrier. Glutamine is produced endogenously in most cell types, but the largest part of circulating glutamine is released by muscle accounting for $65-75 \%$ of plasma glutamine ${ }^{2,3}$. After surgical trauma or during sepsis, plasma glutamine and muscle glutamine concentrations drop despite increased glutamine release by muscle 4,5 . Handling of glutamine by other organs, e.g. the liver and portal drained viscera, can change depending on whether the organism is stressed or not. These phenomena have led to the hypothesis that glutamine can become an essential amino acid under certain conditions ${ }^{6}$. This led to the clinical trials investigating the effects of glutamine supplementation. Despite the fact that Pubmed lists 282 randomized clinical trials concerning therapeutic use of glutamine administration in humans, our knowledge regarding the effects of glutamine supplementation on endogenous glutamine production and consumption rates is still limited.

With regard to the specific effects of glutamine on intestinal mucosa (e.g. improved barrier function), no solid explanations are available. In recent years, however, effects of glutamine on glutathione metabolism have gained interest. Possibly, glutathione defense in the gut wall may be affected by glutamine administration.

Thus the goals of this study were to quantify changes in interorgan amino acid metabolism and organ protein metabolism during glutamine enriched TPN administration. Furthermore, we sought to study the effects of glutamine enriched TPN on gut mucosal GSH synthesis.

\section{MATERIALS AND METHODS}

\section{Animals}

Thirteen female crossbred (Yorkshire x Dutch Landrace) piglets aged 10-12 weeks and weighing $18-22 \mathrm{~kg}$ were used for the study. Before entering the study, the pigs were kept during 1 week in galvanized bar runs $(2 \times 3$ meter) which were equipped with isolated floors and water nipples. The temperature was held between 22 and $25^{\circ} \mathrm{C}$ and the runs were illuminated during 12 hours per day. The food consisted of maintenance pellets for adult sows (Landbouwbelang, Roermond, The Netherlands; $16 \%$ raw protein). They had free access to water.

The study was reviewed and approved by the Animal Ethics Committee of the Maastricht University.

\section{Surgical procedure}

The evening before surgery an enema (A.U.V., Dierenartsencoöperatie Cuyk, The Netherlands) was administered to the animals to prevent postoperative ileus. Premedication consisted of intramuscular administration of azoperone (Stresnil ${ }^{\circledR}, 8 \mathrm{mg} / \mathrm{kg}$; Janssen Pharmaceutica, Tilburg, The Netherlands). After an overnight fast anesthesia was induced with a mixture of $\mathrm{N} 2 \mathrm{O} / \mathrm{O} 2(1: 2)$ and halothane. Lincomycin $(6.25 \mathrm{mg} / \mathrm{kg})$ and spectinomycin $(12.5 \mathrm{mg} / \mathrm{kg})$ 
were given intravenously as antibiotic prophylaxis. Furthermore flunixine (Finadyne, ScheringPlough, Brussels, Belgium) was administered intramuscularly for postoperative analgesia. Fluid resuscitation during surgery consisted of Ringer's lactate (500-1000 mll

The surgical procedure required to produce this model has been described in part previously? In short, after a midline laparotomy 8 catheters (Tygon, Norton Co. Akron, OH, USA) were inserted into various blood vessels in order to be able to sample blood across the organs of interest. Two catheters were inserted into the aorta via the left and right deep iliac circumflex arteries. The tip of one catheter was positioned approximately $5 \mathrm{~cm}$ proximal to the bifurcation (A1), the other above the level of the renal arteries (A2). Similarly two catheters were positioned in the inferior caval vein: one tip lying below (V1) and one above the level of the renal veins (V2). To assess metabolism across the splanchnic organs, 2 catheters were placed in the splenic vein $(S)$, one distally and one was positioned with its tip in the portal vein $(\mathrm{P})$ at the liver hilus. Finally one catheter was inserted into the left hepatic vein $(H)$ by transhepatic puncture. After fluoroscopic control of catheter positions, all catheters were secured with sutures and the application of cyanoacrylate. A gastrostomy catheter was inserted and secured in place with a purse string suture. All catheters were brought out via a tunnel made through the abdominal wall. An illustration of this model can be found in chapter 3 of this thesis. The purpose of the catheters used is described in the section describing the experimental protocol. To be able to take gut mucosal biopsies a Bishop Koop (BK) stoma was constructed ${ }^{8}$. A loop of proximal jejunum with sufficient mesenteric length was transected. A jejunostomy was created using the distal jcjunal loop. This was followed by an end to side anastomosis of the proximal to the distal jejunal loop. The jejunostomy provided access to the jejunum for biopsies. A Foley catheter was inserted into the stoma and inflated carefully to prevent leakage of intestinal contents. Finally the abdominal wall was closed in layers. Total operating time was $4-5$ hours.

\section{Postoperative care}

After surgery, pigs were kept in runs covered with straw with extra heating. lach pig wore a canvass harness to protect the catheters and to allow easy handling of the animal. Stomach retention was monitored every day and drained if necessary. Catheters were checked each day for patency, flushed with heparinized saline and filled with a solution containing a mixture of gentamycin and chymotrypsin to prevent infections or occlusion. Starting on the first postoperative day sow feed was offered. Ten days after surgery the pigs were clinically fully recovered and they were subjected to the first experiment to obtain baseline values (CON).

\section{Tracers}

After baseline blood samples were taken, a primed constant infusion of the following tracers was started: para-aminohippuric acid (PAH; $25 \mathrm{mM}, \mathrm{A} 1422$, Sigma Chemical Co.) through the $\mathrm{A} 1$ and the $\mathrm{S}$ catheter at a rate of $2 \mathrm{ml} / \mathrm{kg} / \mathrm{h}$ after a 1 hour prime ${ }^{7}, \mathrm{~L}-5-15 \mathrm{~N}$ glutamine (prime: $0.78 \mu \mathrm{mol} / \mathrm{kg}$, infusion: $0.78 \mu \mathrm{mol} / \mathrm{kg} / \mathrm{h}$ ), L-[ring $2 \mathrm{H} 5$ ] phenylalanine (prime: $3.04 \mu \mathrm{mol} / \mathrm{kg}$ infusion: $3.04 \mu \mathrm{mol} / \mathrm{kg} / \mathrm{h}$ ), L-1.13C valine (prime: $9.6 \mu \mathrm{mol} / \mathrm{kg}$, infusion: $9.6 \mu \mathrm{mol} / \mathrm{kg} / \mathrm{h}$ ) and L-2.13C glycine (prime: $50 \mu \mathrm{mol} / \mathrm{kg}$, infusion: $50 \mu \mathrm{mol} / \mathrm{kg} / \mathrm{h}$ ). All stable isotopes were purchased from Mass Trace (Woburn, MA, USA) with the exception of the glutamine tracer which was purchased from Cambridge Isotope Laboratories (Woburn, MA, USA). 


\section{Experimental protocol}

After an overnight fast, the pigs were transferred to a small movable cage were the experiment was performed under unrestrained, conscious conditions. After weighing the animal, baseline blood samples were drawn from the arterial catheter. PAH was infused through the catheters in the abdominal aorta (AI) and the splenic vein (S). Stable isotopes were infused through the catheter V2. After 2 hours, when isotopic steady state was obtained 9.10 , blood samples were taken from the various catheters and a mucosal biopsy was taken through the jejunostomy. Tracer infusions were stopped with the exception of the glycine tracer which continued for 4 more hours, after which another biopsy was taken from the gut mucosa. Simultaneously blood samples were taken.

Blood samples for PAH measurements were taken in triplicate. Hematocrit was determined of all blood samples. After receiving total parenteral nutrition for seven days, these experiments were repeated during administration of TPN.

Table $1 \quad$ Composition of aminoacid solutions

Expressed as grams of amino acids per $1000 \mathrm{ml}$. GT is glutamine enriched solution and ST is the control solution

\begin{tabular}{lll}
\hline Aminoacids & GT & ST \\
\hline Isoleucine & 1.6 & 2.0 \\
Leucine & 2.2 & 2.8 \\
Lysine & 2.6 & 3.2 \\
Methionine & 1.6 & 2.0 \\
Phenilalanine & 1.7 & 2.8 \\
Tyrosine & 0.7 & 0.1 \\
Threonine & 1.6 & 2.0 \\
Tryptophan & 0.5 & 0.7 \\
Valine & 2.1 & 2.6 \\
Alanine & 4.6 & 5.7 \\
Arginine & 3.2 & 4.0 \\
Asparagine & 1.0 & 1.2 \\
Glutamate & 1.6 & 2.0 \\
Histidine & 2.0 & 2.4 \\
Proline & 2.0 & 2.4 \\
Serine & 1.3 & 1.6 \\
Glycine & 3.2 & 2.8 \\
Glutamine & 5.7 & 0.0 \\
\hline
\end{tabular}




\section{Nutrition}

After the control experiments, pigs were randomized to receive either a glycyl-glutamine supplemented TPN solution (GT) based on Glamin (B) (Fresenius Kabi) or an isonitrogenous, isocaloric control solution (ST) based on Vamin 18EF(i) (Fresenius Kabi). Randomization was performed by the pharmacist preparing the solutions. The investigator was blinded for the nutrition composition administered. Composition of the nutrition is detailed in table 1 . These young and growing pigs require large amounts of protein. In parallel with their usual enteral feedings, the TPN was administered at a rate of 7 grams of protein $/ \mathrm{kg} /$ day. The amino acid solution was mixed with glucose $50 \%$ and a lipid solution (Intralipid 20\%, Pharmacia \& Upjohn, Sweden). In this mixture lipids provided approximately $40 \%$ of the calories administered. The nitrogen/kcal ratio averaged 1:150. Electrolytes and vitamins were added to the TPN solutions (table 1).

\section{Sample processing}

Immediately after sampling, blood was distributed in heparinized tubes on ice. For determination of PAH $300 \mu \mathrm{L}$ of whole blood was added to $000 \mu \mathrm{L}$ of trichloroacetic acid $10.7 \mathrm{mmol} / \mathrm{L})$. After thorough mixing and centrifugation, the supernatant was collected for further analysis. Centrifugation was performed at $4^{\circ} \mathrm{C}$ for 5 minutes at $8.500 \mathrm{~g}$ to obtain plasma. For determination of glucose, lactate, ammonia and urea $900 \mu 1$. plasma was added to $90 \mu \mathrm{L}$ of a $50 \%$ trichloroacetic solution. For amino acid analysis plasma was deproteinized by addition of $500 \mathrm{\mu L}$ plasma to $20 \mathrm{mg}$ dry sulfosalicylic acid. Native plasma was stored for enrichment analysis of the various tracers. Mucosal biopsies were rinsed in ice cold normal saline and after removing excess water with filter paper the biopsies were snap frozen in liquid nitrogen. At the end of the experiment, wet weight of the biopsies was recorded and the biopsies were added to a vial containing $2.5 \%$ sulfosalicylic acid and glass beads. The mucosa was homogenized using a Mini Beadbeater (Biospec products, Bartlcsville, USA) and frozen in liquid nitrogen. Finally all samples were stored at $\ldots 80^{\circ} \mathrm{C}, \mathrm{c}$ until analysis.

\section{Biochemical analysis}

Glucose, lactate, ammonia, urea and PAH were measured spectrophotomctrically on a Cobas Mira S (Roche Diagnostica, Hoffman-La Roche, Basel, Switzerland) by standard enzymatic methods using commercially available kits as described previously ". Plasma amino acid concentrations were measured on a fully automated High Performance Liquid ('hromatography system as described before ${ }^{12}$. Isotopic enrichments of phenylalanine and valine were measured on a GC-MS (Finnigan Incos XI, San Jose, CA, USA). Glutamine enrichments were determined on a GC-C-IRMS as described previously 13. In short, glutamine was extracted from deproteinized plasma samples using an ion-exchange column. The eluted sample was freeze dried and derivatized with $n$-tert-butyldimethylsilyl-M-methyl-trifluoracetamide after which the sample was injected into the GC-C-IRMS. Glycine and glutathione enrichments were measured on a fully automated liquid chromatography-mass spectrometry system (LC-MS, Thermoquest LCO, Veenendaal, The Netherlands) ${ }^{10}$. Isotopic enrichments were corrected for background ( $=$ pre tracer infusion) enrichments and expressed as tracer to tracee ratio's (TTR). 


\section{Calculations}

$\alpha$-Amino nitrogen was calculated as the sum of the individual amino acids measured 14 . Branched chain amino acids were calculated as the sum of leucine, isoleucine and valine concentrations. The essential amino acids were determined as the sum of histidine, threonine, isoleucine, leucine, valine, tryptophan, methionine, lysine and phenylalanine. Fractional extraction of glutamine across the portal drained viscera was calculated as (arterial glutamine concentration - portal vein glutamine concentration) / arterial glutamine concentration * $100 \%$.

After converting whole blood PAH concentrations to plasma concentrations with formula (1)

(1) PAH plasma $=$ PAH blood * $100 /(100-$ hematocrit $)$

plasma flow was calculated using the dilution of the indicator $\mathrm{PAH}^{7}$.

(2) Flow plasma $=\mathrm{I} /(\mathrm{PAH}] \mathrm{v}-[\mathrm{PAH}] \mathrm{a})$

I represents the rate of infusion of PAH. [PAH]v and [PAH]a are the venous and arterial PAH concentrations in plasma respectively.

Substrate flux (nmol $/ \mathrm{kg}$ body weight $/ \mathrm{min}$ ) was expressed as the net balance across the specific organ and was calculated by multiplying the plasma flow with the venous $[\mathrm{V}]$ minus arterial $[\mathrm{A}]$ substrate concentration ${ }^{3}$. A positive flux indicates net release whereas a negative flux reflects net uptake of the substrate across the organ. Splanchnic flux was calculated by multiplying the mean hepatic plasma flow with the hepatic vein - arterial concentration differences. Liver flux was subsequently calculated as the difference between splanchnic and portal fluxes.

By using tracers protein synthesis and degradation rates in organs can be calculated with the 2-pool model as described by Barrett and Gelfand ${ }^{15}$. The tracer net balance was calculated in an analogous way to substrate net balance by subtracting the amount of enriched amino acid in the venous compartment from the arterial compartment 4 .

(3) $\mathrm{NB}=$ flow * $([\mathrm{V}]-[\mathrm{A}])$

(4) $\mathrm{nb}=$ flow * $\{(|\mathrm{A}| *$ TTRa $)-(\mathrm{V} * \mathrm{TTRv})\}$

The rate of disappearance (Rd) reflects loss of the tracer either by incorporation into protein or (oxidative) degradation and is calculated with formula (5).

(5) $\mathrm{Rd}=\mathrm{nb} / \mathrm{TTRv}$

Since muscle and intestinal tissues possess little phenylalanine hydroxylase activity, Rd of phenylalanine in these tissues represents protein synthesis. The isotopic enrichment in the venous compartment is assumed to represent intracellular enrichment. The liver however, possesses significant phenylalanine hydroxylase activity rendering use of the 
phenylatanine tracer for protein synthesis determinations unreliable $10.1 \%$. Valine, on the other hand, appears to be used mainly for protein synthesis in the liver because its degradation rate in the liver is small18. Therefore valine rates of disappearance are used for determination of liver protein synthesis.

Since the net balance of substrate across an organ is determined by the difference of production ( $\mathrm{Ra}$ ) and disposal (Rd), the Ra can be calculated using the following formula:

(6) $\mathrm{Ra}=\mathrm{NB}+$ Disposal

Whole body phenylalanine kinetics were calculated as described before by Thompson et al. in formula (7) ${ }^{19}$. Since phenylalanine is an essential amino acid the value obtained represents the whole body rate of protein breakdown.

(7) $\mathrm{Q}=\mathrm{I} / \mathrm{TTR} \mathrm{a}$

Use of a glutamine tracer gives more insight into glutamine kinetics within the specific organs of interest. The formulas given above can be applied in a similar manner: glutamine Rd represents uptake of glutamine by the organ and glutamine Ra releasc of glutamine into the venous compartment. Having calculated rates of organ protein synthesis and degradation, estimates of glutamine oxidative disposal and de novo glutamine synthesis in the specific organs can be made by subtracting the amounts of ylutamine being either incorporated in or released from proteins using the following calculations:

(8) $R d_{g \mid n} 0 x=R d_{\text {gln }}-R d_{\text {phe/val }}$

(9) $\mathrm{Ra} \mathrm{gln}_{\mathrm{n}}$ de novo $=\mathrm{Ra} \mathrm{g}_{\mathrm{gln}}-\mathrm{Ra}$ phe $/$ val

In these formulas, $\mathrm{Rd}$ gin ox represents the amount of glutamine oxidized, Ra plln de novo the fraction of glutamine being synthesized de novo. Rd ,unc,val represents the glutamine used for protein synthesis calculated with phenylalanine tracer (PDV) or valine tracer (liver). Ra phe/val indicates glutamine derived from protein breakdown calculated with phenylalanine (PDV) or valine tracers (liver). For these calculations the glutamine content of protein was estimated to be $6.95 \mathrm{~g} / 100 \mathrm{~g}$ protein ${ }^{20}$. Using the 2 pool model, it is assumed that an isotopic steady state of the tracel within the organ of interest exists. Since it is known that glutamine tracer does not equilibrate readily with the very large muscle glutamine pool, no glutamine kinetics across the hindquarter are calculated ${ }^{13}$.

Glutathione synthesis rates in the intestinal mucosa were determined by measuring the rate at which the glycine tracer is incorporated into the tripeptide. After allowing for reaching isotopic steady state a baseline mucosal biopsy was taken 2 hours after start of tracer infusion. A second biopsy was taken 4 hours later. The intracellular glycine enrichment is assumed to reflect the enrichment of the precursor pool and was calculated as the mean of the intracellular glycine enrichment at $t=2$ and $t=6$. The fractional synthetic rate (FSR) of GSH, expressed in \% per day is then calculated as:

(10) $\mathrm{FSR}_{\mathrm{gsh}}=\left(\mathrm{TTR}_{6}-\mathrm{TTR}_{2}\right) / \mathrm{TTR}_{\mathrm{gly}}$ * 6 * $100 \%$ 
The factor 6 in the comparison multiplies the infusion period of 4 hours to obtain the synthesis rate per day.

Absolute glutathione synthesis rates were subsequently calculated by multiplying the FSR with mucosal glutathione concentrations:

$$
\text { (11) } \mathrm{ASR}_{\mathrm{ggh}}=\mathrm{FSR}_{\mathrm{ggh}} \text { * [GSH] mucosa }
$$

\section{Statistics}

Results are presented as means \pm standard errors of the mean (SEM). Differences in glutamine concentrations and fluxes were directly tested by Mann Whithney U tests. Other parameters were tested first with the Kruskal Wallis test, a non parametric one- way analysis of variance. In case of detected significant differences between groups, a Mann Whitney U test was applied to detect between which groups differences were statistically significant. A p-value of $<0.05$ was considered to be significant.

\section{RESULTS}

Thirteen pigs were randomized, of which seven received glutamine enriched TPN and 6 the control solution. Due to loss of hepatic vein catheter patencies in the ST group, fluxes of the liver are only available for the CON and the GT group. Plasma flows across the hindquarter, PDV and liver are summarized in table 2. No significant changes were detected between groups. Valine isotopic data across the liver were incomplete for both ST and GT groups. However, WbRa of phenylalanine, valine and glutamine data were available for all groups.

Table 2 Ilasma flows across hindquarter, portal drained viscera, liver and kidneys ( $\mathrm{ml} / \mathrm{kg} \mathrm{BW} / \mathrm{min}$ ) CON is the control group, ST standard total parenteral nutrition group and GT glutamine enriched total parenteral nutrition group.

\begin{tabular}{lllll}
\hline & CON & ST & GT & $p$ \\
\hline Hindquarter & $21.4 \pm 2.8$ & $23.8 \pm 4.5$ & $29.6 \pm 2.9$ & \\
PDV & $36.8 \pm 8.3$ & $40.7 \pm 18.6$ & $27.3 \pm 2.2$ & \\
Liver & $41.1 \pm 7.3$ & $43.9 \pm 7.3$ & $71.2 \pm 18.8$ &
\end{tabular}

\section{Whole body fluxes and plasma concentrations}

Whole body rate of appearance rates of phenylalanine increased significantly in the ST group $(p=0.001$ ) as well in the GT group ( $p<0.001$ ) (fig 1) compared to control values. In the ST group phenylalanine infused with the TPN constituted $29 \%$ of the difference with the CON group whereas in the GT group this amounted to $46 \%$. There were no significant differences between the ST and GT groups. Valine whole body Ra was increased in both groups: CON $2133 \pm 10$, ST $4772 \pm 346(p=0.01)$ and GT $4288 \pm 358(\mathrm{p}=0.001)$. Glutamine whole body rate of appearance was only significantly elevated in the GT group: CON $5823 \pm 451$, ST $6507 \pm 797$, GT $11805 \pm 1474(p<0.001)$. Glutamine Ra differed significantly between 


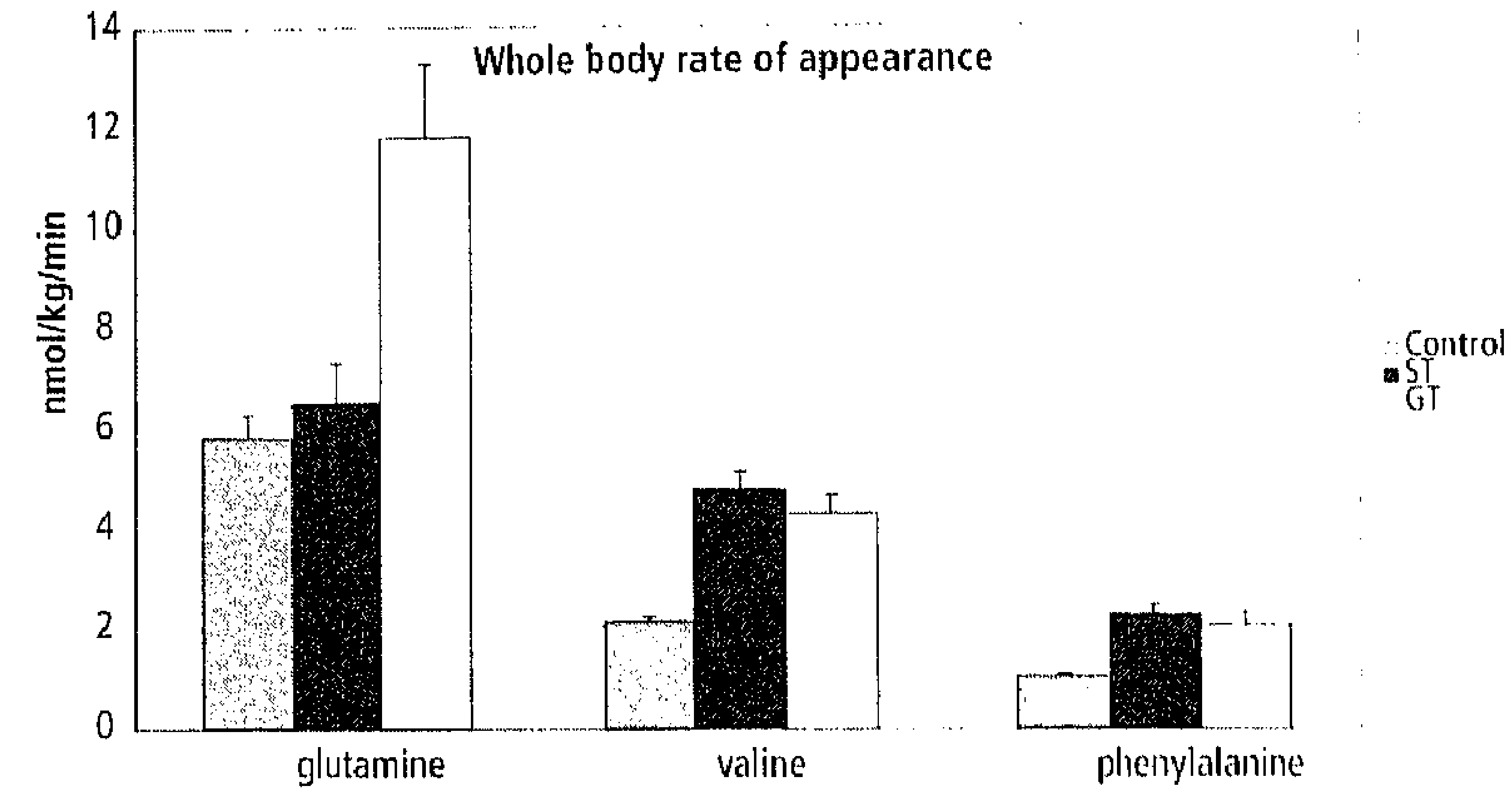

Figure 1 WbRa of glutamine, phenylalanine and valine in postathorptive comlithms and after I week of TPN with or without glutamine IST vis (iT)

the ST and GT group ( $p=0.005)$. The exogenously infused glutamine contributed to approximately $30 \%$ of the difference between the GT and the non glutamine groups.

Glucose and urea concentrations rose in the ST and the GI group (table 3). Ammonia concentrations only increased significantly in the (T) group $(p=0.02)$. Arterial concentrations of most amino acids increased significantly in both GT and ST groups (table 3). (ilutamine concentrations only rose in the GT group (CON $420 \pm 08$ versus (il $400 \pm 22, p=0.05$ ). Arterial glutamine levels were higher in the GT group than in the $55^{\circ}$ group but this difference did not reach statistical significance $(\mathrm{p}=0.07)$.

\section{Hindquarter}

Hindquarter glucose uptake increased and muscle lactate fluxes changed from release to uptake in both TPN groups (table 4). Essential amino acid uptake increased signilicantly across the hindquarter in the GT group versus the CON group (table 4). The ST group showed a similar pattern although this was not statistically significant compared to the CON group. Glutamine efflux increased slightly, but not significantly in both the ST and GT group. Phenylalanine disposal did not change in the GT group versus CON but there was a tendency to a decreased production rate with $30 \%(p=0.075)$ (figure 2). Net phenylalanine release in the control group changed to net phenylalanine retention in the GT group ( $p=0.001$ ) but not in the ST group. No significant changes in muscle protein kinetics or glutamine fluxes occurred in the ST group.

\section{Portal drained viscera}

Glucose consumption by the PDV increased in both TPN groups (table 5). Ammonia production rates simultaneously decreased but this decrease was only significant in the GT group $(\mathrm{p}=0.04)$. The portal drained viscera showed net consumption of the essential amino acids in the CON experiments (table 5). After 1 week of total parenteral nutrition this did not change significantly. Large changes were observed in glutamate, glutamine, citrulline 
Effects of glutamine enriched TPN on interorgan amino acid and gut glutathione metabolism

Table 3 Arterial plasma amino acid concentrations (umol/L). Glucose, lactate and urea concentrations are expressed as mmol/L CON is the control group, ST standard total parenteral nutrition group and GT' glutamine enriched total parenteral nutrition group.

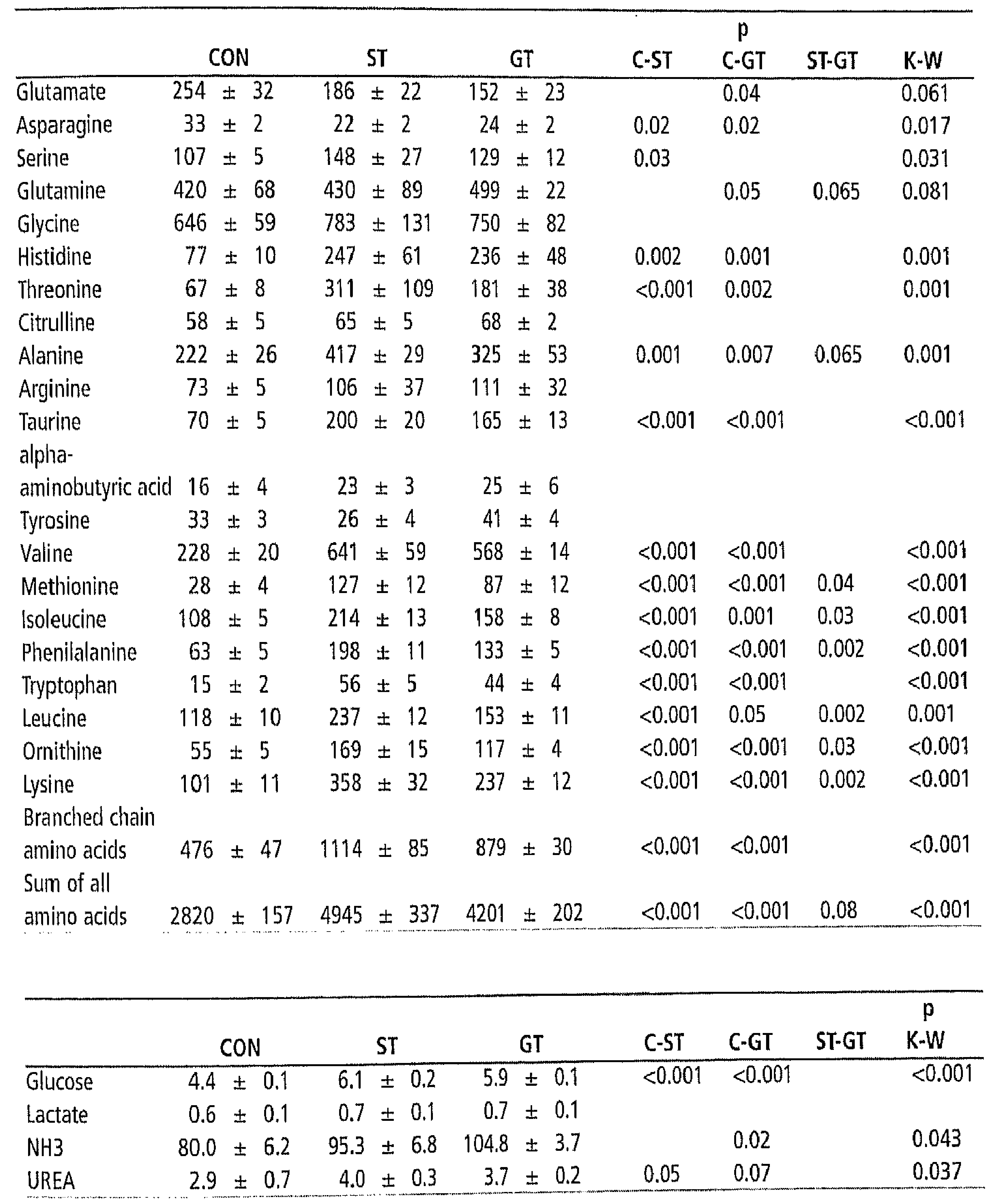

and particularly glycine exchange across the PDV. Glutamate uptake was reduced in the ST group and net glutamate production was observed in GT group ( $\mathrm{p}=0.003$ versus control). 
Chapter 0

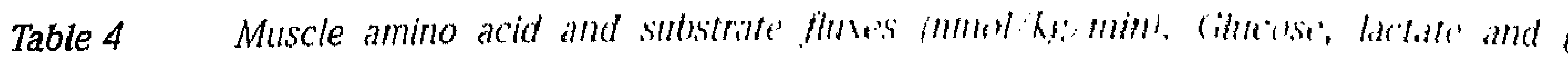

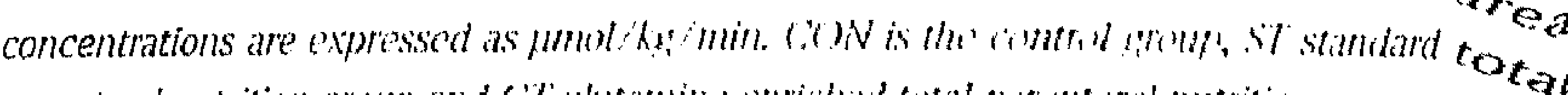

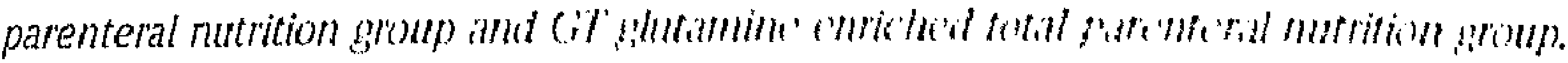

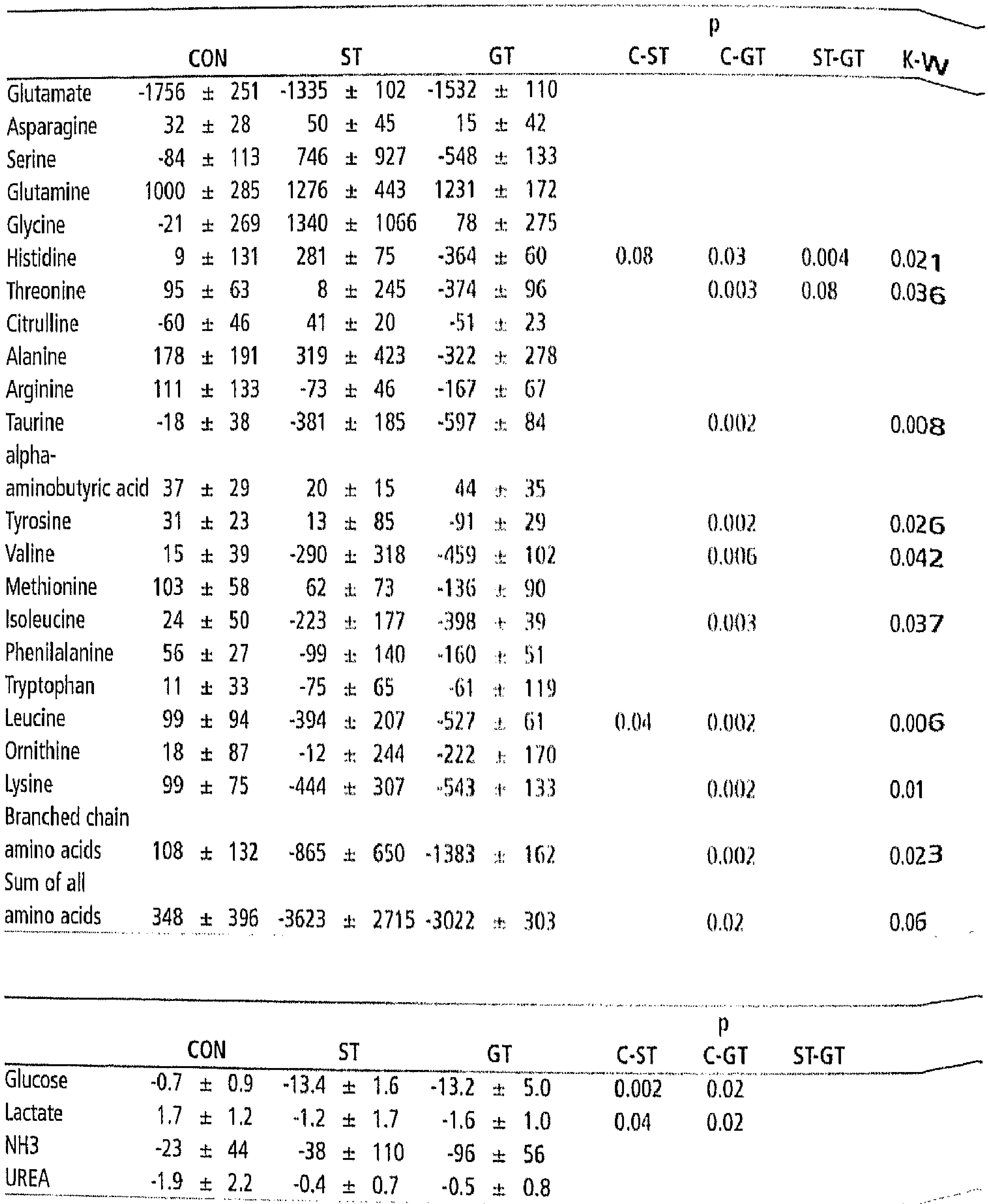

Citrulline production approximately doubled both in the ST and the ( $\mathrm{p}=0.003$ respectively). Glutamine consumption tended to decrease in the ("T group, $\mathrm{b}^{\text {ut }}$ 

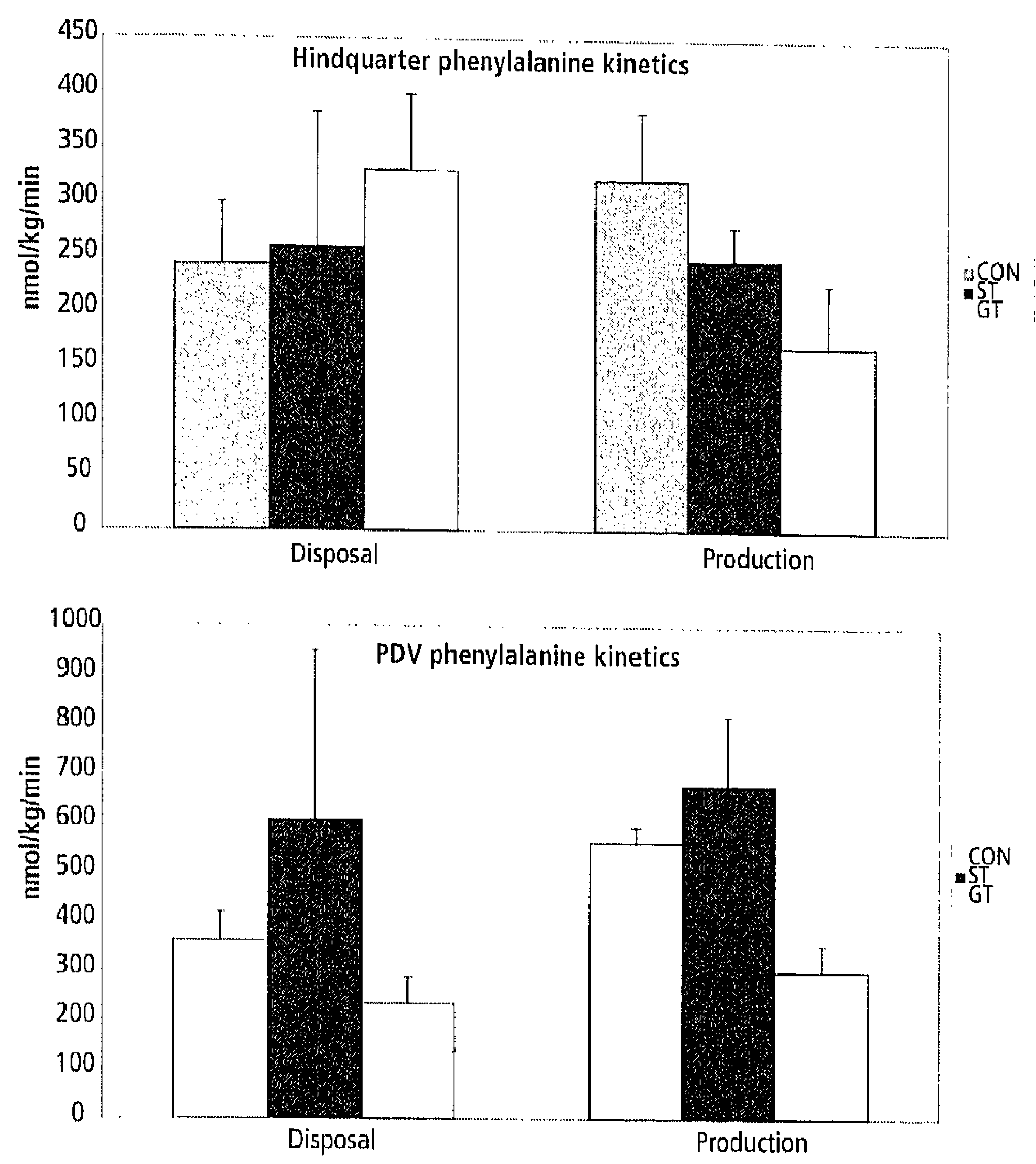

Fig 2 Phenylalanine fluxes across hindquarter and portal drained viscera in nmol/kg body weight/min. CON is the control group, ST standard total parenteral nutrition group and GT glutamine enriched total parenteral nutrition group.

only slightly and not significantly in the ST group (CON $-2190 \pm 288$, ST $-1639 \pm 358$ and GT $-1414 \pm 238, p=0.07)$. Glutamine extraction by the PDV did not differ significantly between groups (CON $15.5 \pm 1.9$, ST $10.9 \pm 2.9$ and GT $10.2 \pm 1.4 \%$ ). Glycine production tended to increase in the GT group $(p=0.06)$ and doubled in the ST group $(p=0.03)$. Phenylalanine production by the PDV decreased significantly from $561 \pm 31$ to $299 \pm 55$ in the GT group. In parallel there was a tendency to decreased phenylalanine disposal (361 \pm 57 versus $234 \pm 53 \mathrm{nmol} / \mathrm{kg} / \mathrm{min}, \mathrm{p}=0.09$ ) in the GT group (figure 2). Glutamine disposal also decreased significantly (CON $3183 \pm 163$, GT $2448 \pm 165$, p=0.02) (figure 3). Glutamine de novo synthesis rates in the PDV increased both absolutely and relatively to total glutamine production rates: CON $187 \pm 57$, GT $582 \pm 169 \mathrm{nmol} / \mathrm{kg} / \mathrm{min}(p=0.04)$; relative to glutamine production rates: CON $18 \pm 5 \%$, GT $87 \pm 4 \%,(p=0.004)$.

\section{Liver}

After administration of glutamine enriched TPN uptake of essential amino acids by the liver appeared to increase, but this was not statistically significant due to large variations (see table 6). 
Table $5 \quad$ PDV amino acid and substrate fluxes ( $\mathrm{nmol} / \mathrm{kg} / \mathrm{min})$. Glucose, lactate and urea concentrations are expressed as $\mu \mathrm{mol} / \mathrm{kg} / \mathrm{min}$. CON is the control group, ST standard total parenteral nutrition group and GT glutamine enriched total parenteral nutrition group.

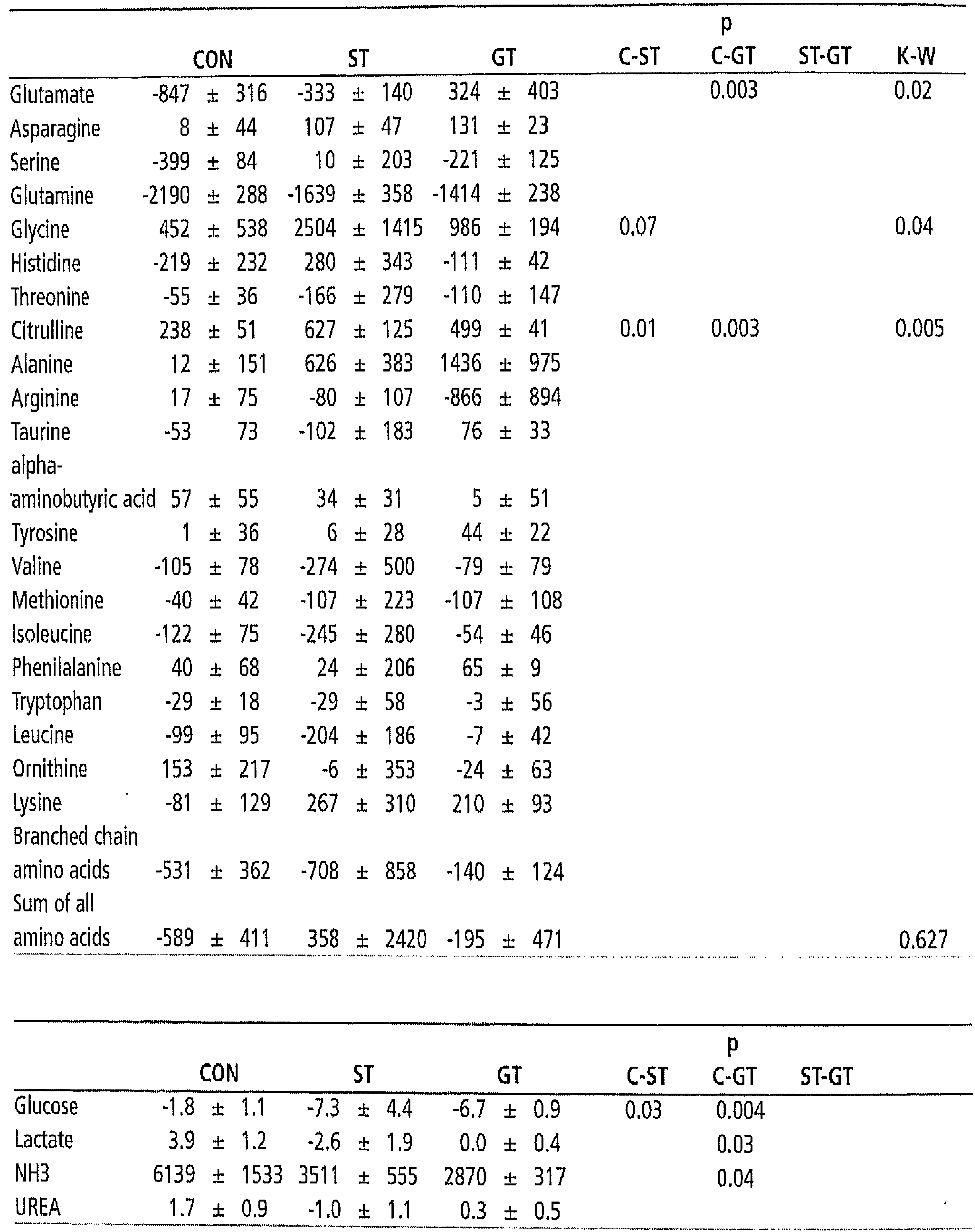

Similarly, glycine uptake by the liver almost doubled but was not statistically significant. In the control experiment glutamine was released by the liver whereas this changed to uptake during 
Table 6

Hepaticamino acid and substrate fluxes $(\mathrm{nmol} / \mathrm{kg} / \mathrm{min})$. Glucose, lactate and urea concentrations are expressed as $\mu \mathrm{mol} / \mathrm{kg} / \mathrm{min}$. CON is the control group and GT the glutamine enriched total parenteral nutrition group.

\begin{tabular}{|c|c|c|c|c|}
\hline & $\mathrm{CON}$ & & GT & $\begin{array}{c}p \\
\mathrm{C}-\mathrm{GT}\end{array}$ \\
\hline Glutamate & $4005 \pm 1228$ & 3196 & \pm 746 & \\
\hline Asparagine & $-140 \pm 58$ & -201 & \pm 22 & \\
\hline Serine & $403 \pm 148$ & -295 & \pm 351 & \\
\hline Glutamine & $851 \pm 597$ & -1257 & \pm 987 & 0.05 \\
\hline Glycine & $-1776 \pm 555$ & -3523 & \pm 1278 & \\
\hline Histidine & $-506 \pm 507$ & -441 & \pm 323 & \\
\hline Threonine & $-69 \pm 75$ & -491 & \pm 320 & \\
\hline Citrulline & $-161 \pm 103$ & -413 & \pm 63 & \\
\hline Alanine & $-1294 \pm 287$ & -2284 & \pm 1119 & \\
\hline Arginine & $-372 \pm 388$ & 10 & \pm 41 & \\
\hline $\begin{array}{l}\text { Taurine } \\
\text { alpha- }\end{array}$ & $-81 \pm 90$ & -449 & \pm 360 & \\
\hline aminobutyric acid & $-26 \pm 63$ & 55 & \pm 76 & \\
\hline Tyrosine & $-177 \pm 73$ & -132 & \pm 75 & \\
\hline Valine & $-145 \pm 105$ & -75 & \pm 316 & \\
\hline Methionine & $-26 \pm 84$ & -408 & \pm 233 & \\
\hline Isoleucine & $12 \pm 118$ & -55 & \pm 187 & \\
\hline Phenilalanine & $-144 \pm 92$ & -414 & \pm 254 & \\
\hline Tryptophan & $.39 \pm 72$ & -102 & \pm 67 & \\
\hline Leucine & $.51 \pm 131$ & -157 & \pm 289 & \\
\hline Ornithine & $-174 \pm 247$ & 97 & \pm 71 & \\
\hline Lysine & $-97 \pm 188$ & -767 & \pm 422 & \\
\hline $\begin{array}{l}\text { Branched chain } \\
\text { amino acids } \\
\text { Sum of all }\end{array}$ & $105 \pm 549$ & -287 & \pm 776 & \\
\hline amino acids & $-1005 \pm 616$ & -2910 & \pm 2371 & \\
\hline & $\mathrm{CON}$ & & GT & C-GT \\
\hline Glucose & $14.0 \pm 3.2$ & 22.6 & \pm 12.9 & \\
\hline Lactate & $-13.9 \pm 5.8$ & -2.5 & \pm 2.6 & 0.02 \\
\hline $\mathrm{NH} 3$ & $-7674 \pm 1590$ & -4259 & \pm 722 & \\
\hline UREA & $0.9 \pm 0.7$ & 6.4 & \pm 4.6 & \\
\hline
\end{tabular}

administration of glutamine enriched TPN ( $p=0.05)$. Fluxes of glucose, ammonia and urea did not change significantly. Lactate consumption decreased significantly in the GT group. No statistically significant changes in glutamine kinetics were observed across the liver (fig. 3) 

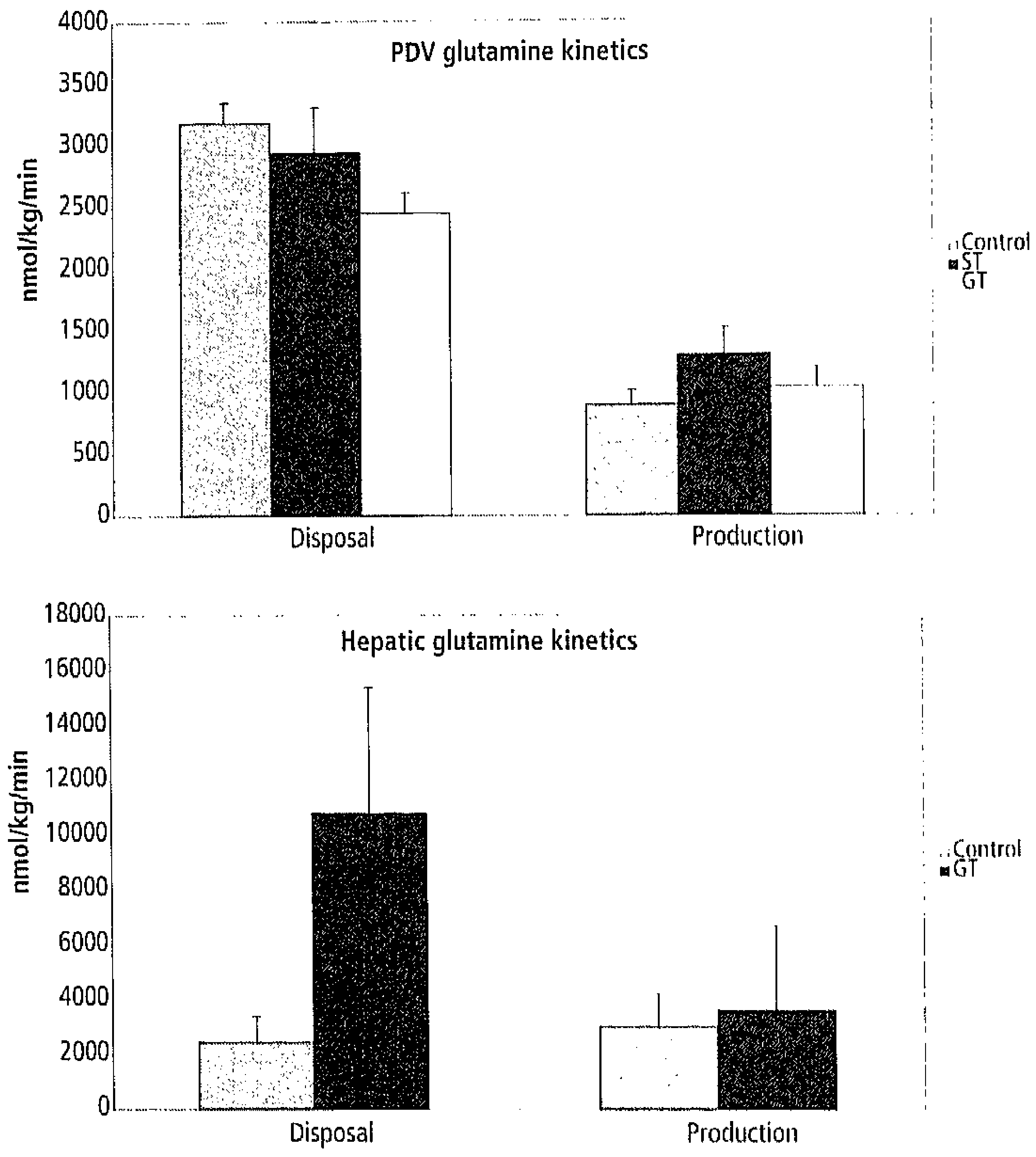

Figure 3 Effects of TPN on glutamine kinetics across the PIDV and liver

$S T$ is the standard total parenteral nutrition group and (it the glutumine s'mriche'd total parenteral nutrition group.

\section{Intestinal mucosa}

In contrast to arterial amino acid concentrations, mucosal glutamine levels were lower in the GT group compared to the ST group (table 7). A similar difference was found between the GT and CON group although this was statistically not significant $(p=0.085)$. Other amino acid concentrations are summarized in table 6 . Glutathione concentrations were lower in both TPN fed groups versus CON (table 8). Glutathione synthesis rates in jejunal mucosa, however, were not significantly different.

\section{DISCUSSION}

In this study we demonstrated that glutamine supplementation to TPN during 1 week in a porcine model caused differences both in organ protein and interorgan glutamine metabolism. 
Effects of glutamine enriched TPN on interorgan amino acid and gut glutathione metabolism

Table 7 Mucosal amino acid concentrations ( $\mu m$ mol/L). CON is the control group, ST standard total parenteral nutrition group and GT glutamine enriched total parenteral nutrition group.

\begin{tabular}{|c|c|c|c|c|c|c|c|c|c|}
\hline & & CON & & ST & GT & C-ST & C-GT & ST-GT & $\mathrm{K}-\mathrm{W}$ \\
\hline Glutamate & 3046 & \pm 339 & 2565 & \pm 638 & $2462 \pm 438$ & & & & \\
\hline Asparagine & 75 & \pm 10 & 94 & \pm 20 & $73 \pm 12$ & & & & \\
\hline Serine & 1087 & \pm 137 & 924 & \pm 284 & $958 \pm 201$ & & & & \\
\hline Glutamine & 263 & \pm 40 & 222 & \pm 77 & $198 \pm 36$ & & 0.085 & 0.035 & 0.077 \\
\hline Histidine & 88 & \pm 10 & 69 & \pm 23 & $79 \pm 14$ & $<0.001$ & 0.04 & & 0.004 \\
\hline Glycine & 1738 & \pm 175 & 1473 & \pm 354 & $1303 \pm 217$ & & & & \\
\hline Threonine & 484 & \pm 59 & 387 & \pm 111 & $382 \pm 68$ & 0.001 & 0.008 & & 0.003 \\
\hline Citrulline & 204 & \pm 28 & 191 & \pm 46 & $199 \pm 34$ & & & & \\
\hline Alanine & 118 & \pm 19 & 127 & \pm 22 & $87 \pm 25$ & & & & \\
\hline Arginine & 431 & \pm 68 & 402 & \pm 99 & $341 \pm 48$ & & & & \\
\hline $\begin{array}{l}\text { Taurine } \\
\text { alpha- }\end{array}$ & 1888 & \pm 235 & 1520 & \pm 403 & $1430 \pm 300$ & & & & \\
\hline aminobutyric aci & id 53 & \pm 8 & 64 & \pm 10 & $26 \pm 8$ & & & & \\
\hline Tyrosine & 42 & \pm 5 & 43 & \pm 11 & $32 \pm 5$ & & & & \\
\hline Valine & 279 & \pm 36 & 301 & \pm 66 & $235 \pm 50$ & 0.001 & 0.004 & 0.07 & $<0.001$ \\
\hline Methionine & 78 & \pm 9 & 101 & \pm 30 & $56 \pm 10$ & & & & \\
\hline Isoleucine & 327 & \pm 34 & 400 & \pm 63 & $265 \pm 57$ & & & & \\
\hline Phenilalanine & 172 & \pm 21 & 219 & \pm 34 & $141 \pm 29$ & 0.007 & & 0.02 & 0.01 \\
\hline Tryptophan & 258 & \pm 41 & 475 & \pm 73 & $234 \pm 54$ & & & & \\
\hline Leucine & 246 & \pm 18 & 102 & \pm 24 & $94 \pm 20$ & & & & \\
\hline Ornithine & 58 & \pm 6 & 114 & \pm 17 & $44 \pm 9$ & 0.03 & & & 0.043 \\
\hline $\begin{array}{l}\text { Lysine } \\
\text { Sum of all }\end{array}$ & 95 & \pm 10 & 77 & \pm 19 & $74 \pm 13$ & 0.003 & & 0.07 & 0.009 \\
\hline amino acids & 11486 & \pm 1290 & 875 & \pm 2507 & $9339 \pm 1757$ & & & & \\
\hline
\end{tabular}

Table 8 Gut mucosal GSH concentrations, fractional and absolute synthesis rates. CON is the control group, ST'standard total parenteral nutrition group and GT glutamine enriched total parenteral nutrition group.

\begin{tabular}{lcccccccc}
\hline & CON & \multicolumn{1}{c}{ ST } & GT & C-ST & C-GT & ST-GT & K-W \\
\hline GSH & $1335 \pm 143$ & 1135 & \pm 239 & $1082 \pm 213$ & 0.05 & 0.07 & & 0.064 \\
FSR & $190 \pm 25$ & $274 \pm 102$ & $295 \pm 52$ & & & & \\
ASR & $3177 \pm 389$ & $3780 \pm 1265$ & $4024 \pm 717$ & & & & \\
\hline
\end{tabular}

\section{Protein metabolism}

Whole body phenylalanine rate of appearance increased during administration of parenteral nutrition. This was mainly due to an increased protein turnover, whereas $29-46 \%$ of this 
increase could be explained by the exogenously infused phenylalanine. Whole body protein breakdown increased with $52 \%$ in the GT and $84 \%$ in the ST group. There was no significant difference between the ST and GT group. This is consistent with isotopic data from catabolic animal and human studies, which did not show an effect of glutamine supplementation on whole body protein turnover ${ }^{21-23}$. At the organ level, however, differences in protein metabolism occurred due to glutamine administration. In muscle net protein breakdown changed to protein retention in the GT group as indicated by an increased uptake of essential amino acids and the phenylalanine tracer data. The phenylalanine tracer results suggested this to result from a reduced production rate of phenylalanine reflecting a decrease in protein breakdown rather than changes in protein synthesis. Others have also reported enhanced muscle protein anabolism, but mostly based on (measures of) enhanced protein synthesis rates in muscle biopsies22, 24-20. However, decreased muscle protein breakdown due to glutamine supplementation has been reported before 22 .

Within the portal drained viscera glutamine supplementation decreased protein turnover and in particular protein breakdown. Although in vitro experiments suggested otherwise ${ }^{27}$, glutamine supplementation in different animal models resulted in unchanged mucosal protein fractional synthetic rates 28.30 . Enteral glutamine administration in healthy humans, treated with glucocorticoids, yielded similar results.31. In dogs treated with glucocorticoids, however, glutamine did increase mucosal protein synthesis rates? 23 . On the other hand, glutamine depletion did not affect gut amino acid metabolism '", and gllutamine did not increase intestinal protein content 32 . Decreased protein breakdown rates have not been reported before to our knowledge and may have been influenced by the absence of catabolic stress and the long duration of administration of TPN prior to the measurements.

In both the ST and GT groups the large fluxes of glycine across the PDV were striking. Possibly, recycling of glycine used for conjugation of bile acids by the liver explains this phenomenon ${ }^{33}$. Both glycine and taurine are used for conjugation of bile acids. In pigs the ratio of these amino acids used for this process is more than a factor 9 94 . Increased enterohepatic cycling of bile acids which are conjugated with glycine in the liver to be deconjugated subsequently in the gut, where glycine and the bile acids are reabsorbed can explain our observations. The observed increase in glycine uptake by the liver, though not significant, fits with this hypothesis. In a model similar to the one used in the present study, lower glycine fluxes were reported during enteral feeding 35 . This suggest that either prolonged enteral starvation or administration of total parenteral nutrition per se increases the glycine flux across the PDV.

Although an increased uptake of essential amino acids was observed across the liver in the GT group, large variations precluded detection of significant changes. Unfortunately, insufficient data were available to determine valine kinetics across the liver. However, increased protein synthesis rates in liver due to glutamine enriched TPN have been reported22.

Thus, long term administration of TPN resulted in increased protein turnover rates at the whole body level. Glutamine enriched TPN, but not standard TPN increased net protein synthesis in muscle. On the other hand, glutamine administration decreased protein turnover and net amino acid retention in the portal drained viscera. 


\section{Glutamine metabolism}

Although plasma glutamine concentrations and glutamine rate of appearance increased after administration of TPN, gut glutamine uptake and mucosal glutamine concentration decreased in the glutamine enriched group. Fractional extraction rates did not change with administration of total parenteral nutrition nor with glutamine supplementation. The absolute fractional extraction rates varied around $10-15 \%$ which are lower compared to reported values in rats and humans $s^{30.38}$, but studies in dogs 39 and pigs ${ }^{40}$ provided similar extraction rates. Possible explanations include beside obvious interspecies differences, also differences in sampling since the rat and human studies primarily used blood from the proximal gastrointestinal tract whereas the latter animal models used portal venous samples. Associated with lower flow rates across the PDV, glutamine uptake tended to decrease in the GT group. The tracer data indicated that this coincided with a decrease in glutamine disposal and an unchanged glutamine production rate. This is in contrast to data which showed increased uptake by the gut or PDV with increasing plasma glutamine concentrations $36-38,41,42$. However, most of these data were obtained under postabsorptive and catabolic conditions. Indeed, data in animal models during metabolically stable conditions are in accordance with our findings that glutamine uptake by the PDV does not increase with additional glutamine 43,44 . Apparently in the non-stressed animal the intestine does not require more glutamine, despite the fact that no enteral nutrition is given.

Along with most of the other intracellular free amino acid concentrations, glutamine mucosal glutamine concentrations were lower in the glutamine supplemented group. This was associated with a reduced protein turnover in the gut, decreased glutamine uptake and a reduced ammonia production. This suggests that a more complex mechanism is active to down regulate certain aspects of protein and amino acid metabolism in the gut rather than that these phenomena can be explained by solitary effects on isolated enzymes such as glutaminase activity, which do exist 30 . 45. We speculate that changes in cellular hydration status and membrane potential may be implicated in the observed changes in glutamine and protein metabolism 46,47 .

To our knowledge no isotopic data have been reported previously with regard to glutamine production and degradation rates by the gut during glutamine enriched TPN. Within the PDV glutamine de novo synthesis rates increased, whereas oxidation rates remained unchanged. The effect on oxidation rates is consistent with the effects of glutamine on gut glutaminase activity ${ }^{48}$. Although glutamine synthesis rates in the gut are limited as glutamine synthetase activities in the gastrointestinal tract are low ${ }^{49}$, they are not negligible as demonstrated by our data. It might bethat the upregulation of de novo glutamine synthesis is a means to counteract the consequences of decreased glutamine uptake by the gut, analogous to the situation in septic animals 50 .

The liver changed from net release to significant uptake of glutamine in the glutamine supplemented group. Therefore, it appears that, in contrast to what has been assumed, addition of glutamine to TPN does not result in an increased gut glutamine consumption ${ }^{51}$, but is metabolized by the liver. This confirms data from an earlier study in fasting dogs 44 .

Intracellular glutamine concentrations have been hypothesized to stimulate muscle protein synthesis rates 52 , 53. Increased muscle glutamine concentrations due to glutamine supplemented TPN 54,55 , could therefore account for the observed changes in muscle 
protein metabolism. Others, however, have not found increased muscle glutamine concentrations with glutamine administration in critically ill patients ${ }^{\text {St }}$. Our data indicate that glutamine supplementation did not decrease muscle glutamine release. Similar findings have been reported by others 44,57 . Unchanged glutamine release from the hindquarter in the presence of net protein retention, indicates enhanced de novo glutamine synthesis rates. A sustained shrinking glutamine pool is not a likely option after 1 week of TPN. Because glutamine destabilizes glutamine synthetase ${ }^{58}$, this would indicate that muscle glutamine concentrations have been decreased rather than increased in our study.

Analogous to the decrease in most mucosal intracellular free amino acids, mucosal glutathione concentrations decreased although synthesis rates remained similar. Apparently, glutamine is not a limiting factor for glutathione synthesis in the gut during TPN administration in the absence of a catabolic insult.

In conclusion, infusion of TPN increases whole body protein turnover and has anabolic effects on muscle and intestinal tissue. Addition of glutamine to TPN results in enhanced protein deposition in the muscle. Protein synthesis in the gut decreased, but the relative decrease in proteolysis was somewhat larger. Despite increased arterial plasma glutamine concentrations, glutamine enriched TPN did not change muscle glutamine release. Furthermore, it did not increase but rather decreased gut glutamine consumption and mucosal glutamine concentrations in the presence of a lower (although statistically not significant) extraction rate. This was accompanied by an increased de novo glutamine synthesis in the PDV. The supplemental glutamine was not metabolized by the gut, but rather processed by the liver. Glycine fluxes across the portal drained viscera increased significantly by administration of TPN. Presumably this is due to an increased enterohepatic cycling of bile acids which are conjugated to glycine. Finally, no significant effects of glutamine enriched parenteral nutrition on glutathione concentrations or synthesis rates in intestinal mucosa were observed. 


\section{REFERENCES}

I Newsholme P, Procopio I, Lima M M, Pithon-Curi T C and Curi R. Glutamine and glutanate -their central role in cell metabolism and function. Cell Biochem Funct. 2003; 21: 1.9.

2 Stumvoll MW, I'erriello Gi, Nurihan N, Bucci A. Welle S, Jansson P A, Bier D M, Jenssen T and Gerich J E. Cilutamine and alanine metabolism in NIDDM. Diabetes. 1996; 4: 863-808

3 Nurihan N, Bucci $A$, P'eriello $G$, Sumvoll M, Dailey G, Bier D M, Toft I, Jenssen T G and Gerich J E. Glutamine: a major gluconeogenic precursor and vehicle for interorgan transport in man. J Clin Invest. 1005; 105:272.277

4 Karinch A M, Pan M, L.in C. M, Strange R and Souba W W. Glutamine metabolism in sepsis and infection. J Nutr: 2001; 131:2535525385

5 van Acker B A C, Hulsewé K W E. Wagenmakers A I M, von Meyenfeldt M F and Soeters P B. Butamine appearance rate in plasma is not increased after gastrointestinal surgery in humans. Nutrition. 2000; 130: $1560 \cdot 1571$

- l.acey I M and Wimore D) W. Is glutamine a conditionally essential amino acid? Nutr Rev 1900; 48: $247.30 \%$

7 Have ten C: A M, Bost M C. F, Suyck-Wierts (.) A W, Bogaard van den A E J M and Deulz N F. P. Simultancous tncasurement of netabolic flux in portally-drained viscera, liver, spleen, kidney and hindquanter in the conscious pig. Laboratory Animals. 1996; 30: 347-358

8 Bishop 11 ( $;$ and Koop C. I: Management of meconium ileus: resection, Roux-en-Y anastomosis and ilenstomy irrightion with pancreatic enzymes. Ann Surg. 1957; 145: 410

1) Denl1\% N J:, Bruins M I and Socters P B. Infusion of soy and casein protein meals affects interorgan animo acid metabolism and urea kinetics differently in pigs. J Nutr: 1998; 128: 2435.2445.

10 van li:jk It M, Rowyakkers I) $R$, Soeters P' $B$ and Deutz N E. Determination of amino acid isotope enrichment using liquid chromatuyraphy-mass spectrometry. Anal Biochem. 1000; 271:8-17

11 Heencman $S$ and Deutz. $N$ L. Fiffects of decreased glutamine supply on gut and liver metabolism in vivo in rats. (lim sici llondl. 1908; 85: 437-444.

12 vanl lijk H M, van der Heijden M A, van Berlo C L and Soeters P B. Fully automated liquidchromatopraphic detomination of amino acids. Clin Chem. 1988; 34: 2510.2513

13 Van Acker B A C., Hulscwé K W E, Wagenmakers A J M, Deutz N E P, Van Kreel B K, Halliday D, Mathows D E, Soeters P'B and Von Meyenfeldt M F. Absence of glutamine isotopic steady state: implications for the assessment of whole-body glutamine production rate. Clin Sci /Colch/. 1998; 95: 339.340

14 van Fijik $/ 1$ M, Rooyakkers D R and Deutz N E. Rapid routine determination of amino acids in plasma by high-performance liquid chromatography with a $2-3$ microns Spherisorb ODS II column. I Chromatogr: 1993; 620: 143-148

15 Barrett E I, Revkin J H, Young L. H, Zaret B L, Jacob R and Gelfand R A. An isotopic method for measurement of muscle protein synthesis and degradation in vivo. Biochem J. 1987; 245: 223-228

16 Moller N, Meek S, Bigelow M, Andrews I and Nair K S. The kidney is an important site for in vivo phenylalanine-to-tyrosine conversion in adult humans: A metabolic role of the kidney. Proc Natl Acad Sci US A. 2000; 97: 1242-1246.

17 Tourian A, Goddard I and Puck T T. Phenylalanine hydroxylase activity in mammalian cells. $J$ Cell Physiol. 1909; 73: 159-170 
18 Shinnick F L and Harper A E. Branched-chain amino acid oxidation by isolated rat tisste preparations. Biochim Biophys Acta. 1970; 437: 477-480.

19 Thompson $\mathrm{G} N$, Pacy P I, Merritt $\mathrm{H}$, Ford $\mathrm{G}$ C, Read M A, Cheng K $N$ and Halliday l.). Rapid measurement of whole body and forearm protein turnover using a $2 \mathrm{H} 5$ phenylialinine model. $\mathrm{Am}$ I Physiol. 1989; 256: E031-639

20 Hankard R G, Darmaun D, Sager B K, D'Amore D, Parsons $W$ R and Haymond M. Response of

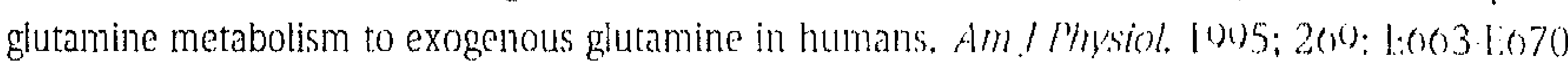

21 Long C L, Nelson K M, DiRienzo D B, Weis I K, Stah R D, Broussard T D), Theus W I., ( Clark I A, Pinson T W, Geiger J W, Laws H L, Blakennore W S and Carraway R P. Cilutamine supplementation of enteral nutrition: impact on whole body protein kinetics and ghucose metabolism in critically ill patients. JPEN I Parenter linteral Nutr: 1905; 10:470-470

22 Naka S, Saito H, Hashiguchi Y, Ming-Tsan I, Furukiwa S, Inaba T, Fukushima R. Wadia N and Muto T. Alanyl-glutamine-supplemented total parenteral nutrition improvess survival and protenin metabolism in rat protracted bacterial peritonitis model. IPl: N I larenter linteral Nutr: $10(0) ; 20: 417.423$

23 Humbert B, Nguyen P, Dumon H, Deschamps I and Darmaun D. Does enteril ylutamine: modulate

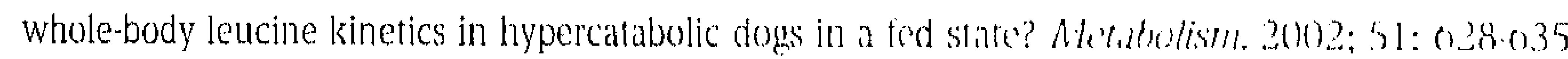

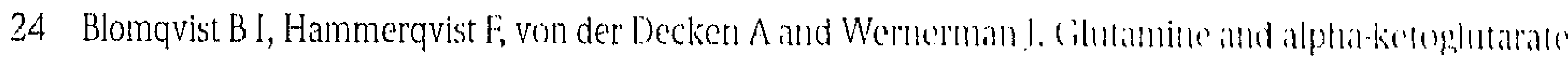
prevent the decrease in muscle free glutamine concentration and influence pronem symbesis atter lotat hip replacement, Metabolism. 1005; 44:1215.1222

25 Watt $\mathrm{P}$ W, Hundal H S, Downie S and kennie M I. C.lutanne increases protein symhessis in heart.

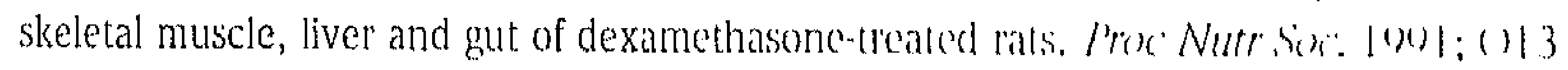

20 Hammarquist F, Wernerman J, von der Decken $\Lambda$ and Vinnars F. Alanyl ylutamine comuteracts the depletion of free glutamine and the postoperative decline in protein synthesis in skeletal musick. Alm Surg. 1900; 212: 637-044

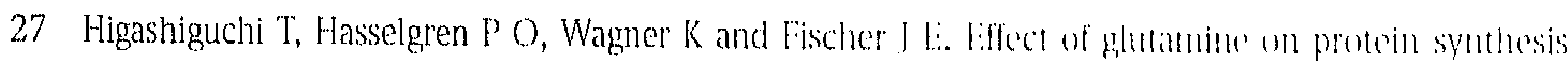
in isolated intestinal epithelial cells. JPEN J Parenter linterial Nutr: 1003: 17:307.31.4

28 Yoshida S, Leskiw M J, Schluter M D, Bush K T, Nagele R (, , I.anza lacoby S and Sicin T I'. L:flect of

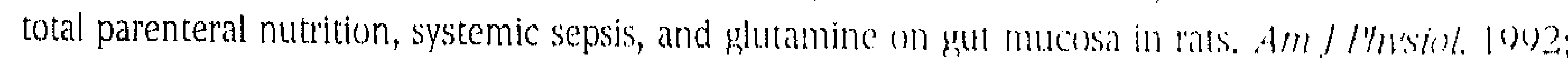
263: $\mathrm{E} 308-373$

29 Marchini J S, Nguyen P, Deschamps J Y, Maugere P, Krempi M and Darmaun D. Lffect of intlavenous

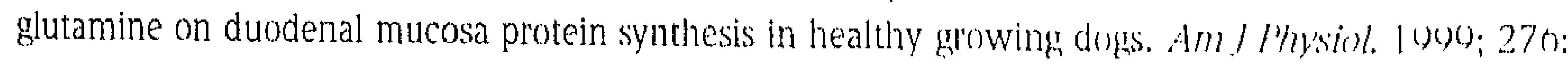
E747.753

30 Kaibara A, Yoshida S, Yamasaki K, Ishibashi N and Kakegawa T. Effect of glutanine and chemotherapy on protein metabolism in tumor-bearing rats. J Surg Res. 1094; 57: 143.144

31 Bouteloup-Demange C, Claeyssens S, Maillot C, Lavoinne A, Lerebours l: and Dechelotte P. Lffects of enteral glutamine on gut mucosal protein synthesis in healthy humans receiving glucocorticoids. Am J Physiol Gastrointest Liver Physiol. 2000; 278: G077-681

32 Shizuka F, Vasupongsotorn S, Kido Y and Kishi K. Comparative effect of intravenously or intragastrically administered glutamine on small intestinal function of the rat. Tokushima. J Exp Med. 1990; 37: 49.57

33 Soeters P B, Hulsewe K W E, Deutz N E P, Luiking Y and Dejong C H C. Gastrointestinal disease. In: L. A. Cynober. Metabolic and therapeutic aspects of amino acids in clinical nutrition. 2. Boca Raton: CRC Press. 2004: 405-418

34 Kuramoto T, Miyamoto J, Konishi M, Hoshita T and Masul T. Bile acids in porcine fetal bile. Biol Pharm Bull. 2000; 23: 1143.1140 
35 Bruins $M$ J, Soeters P B and Deutz N E. Endotoxemia affects organ protein metabolism differently during prolonged feeding in pigs. J Nutr. 2000; 130: 3003-3013

36 Haque S M, Chen K, Usui N, liboshi Y, Okuyama H, Masunari A, Cui L., Nezu R, Takagi Y and Okada A. Alanyl-glutamine dipeptide-supplemented parenteral nutrition improves intestinal metabolism and prevents increased permeability in rats. Ann Surg, 1996; 223: 334-341

37 Souba W W. Glutamine: a key substrate for the splanchnic bed. Annu Rev Nutr. 1991; 11:285-308

38 van der Hulst R R, von Meyenfeldt M F, Deutz N E and Soeters P B. Glutamine extraction by the gut is reduced in depleted patients with gastrointestinal cancer. Ann Surg. 1907; 225: 112-121.

39 Souba WW and Wilmore D W. Postoperative alteration of arteriovenous exchange of amino acids across the gastrointestinal tract. Surgery. 1983; 94:342-350.

40 Bruins M I, Deutz N E P and Soeters P B. Aspects of organ protein, amino acid and glucose metabolism in a porcine model of hypermetabolic sepsis. Clin Sci (Colch). 2003; 104: 127-141

41 Inneberg K, Mjaaland M, Balteskard L, Jenssen T G, Bjoro T and Revhaug A. Both growth hormone and exogenous glutamine increase gastrointestinal glutamine uptake in trauma. Ann Surg. 1997;225: 07.102

42 Deulz N E: B, Reijven I' L., Athanasas G and Soeters P B. Post-operative changes in hepatic, intestinal, splenic and muscle fluxes of amino acids and ammonia in pigs. Clin Sci Colch. 1992; 83: 607-014

43 Karney 1 and Roth $1:$ Influence of alanylglutamine infusion on gastrointestinal glutamine and alanine metabolism in anesthetized dogs. Metabolism. 1989; 38: 73.77

44 Borcl M, Williams P E, Jabbour $K$ and Ftakol] P J. Chronic hypocaloric parenteral nutrition containing gylutamine promotes hepatic rather than skeletal muscle or gut uptake of glutamine after fasting. JPEN I Tarenter Linteral Nutr: 1006;20:25-30

45 Kong S I: Hall I C C Cooper D and MCCauley R D. Glutamine-enriched parenteral nutrition regulates the activity and expression of intestinal glutaminase. Biochim Biophys Acta. 2000; 1475: 67.75

40 Hatssinger 1), Rorth 1: l, ang F and Gerok W. Cellular hydration state: an important determinant of protein catibolism in health and disease. The Lancet. 1993; 341: 1330-1332

47 Wigenmakers A J. Muscle function in critically ill patients. Clin Nutr: 2001; 20:451-454

48 kiong S I:, I Iall I C , C,ooper D and McCauley R D. Glutamine-enriched parenteral nutrition regulates the activity and cxpression of intestinal glutaminase. Biochim Biophys Acta. 2000; 1475: 67-75.

49 Inme's I. A, L.unn P' '., Middleton S and l:lia M. Distribution of glutaminase and glutamine synthetase activities in the human gastrointestinal tract. Clin Sci /Colch/. 1998; 94: 313-319

50 Nognchi $Y$. lames I H, Fischer J E and Hasselgren $\mathrm{P} O$. Increased glutamine consumption in small intestine epithelial cells during sepsis in rats. Am J Surg. 1990; 172: 199-205

51 Souba W W, Klimberg V S, Plumley D A, Salloum R M, Flynn T C, Bland K I and Copeland E M d. The role of glutamine in maintaining a healthy gut and supporting the metabolic response to injury and infection. I Surg Res. 1990; 48: 383.391.

52 Jepson M M, Bates P C, Broadbent P, Pell J M and Millward D J. Relationship between glutamine concentration and protein synthesis in rat skeletal muscle. Am J Physiol. 1988; 255: E1 106-172

53 Rennie M J, Hundal H S, Babij P, MacLennan P, Taylor P M, Watt P W, Jepson M M and Millward D J. Characteristics of a glutamine carrier in skeletal muscle have important consequences for nitrogen loss in injury, infection, and chronic disease. Lancet. 1986; 2: 1008-1012

54 Hammarqvist F, Sandgren A, Andersson K, Essen P, McNurlan M A, Garlick P J and Wernerman J. Growth hormone together with glutamine-containing total parenteral nutrition maintains muscle glutamine levels and results in a less negative nitrogen balance after surgical trauma. Surgery. 2001; 129:576-586 
55 Stehle P, Zander J, Mertes N, Albers S, Puchstein C, Lawin P and Furst P. Effect of parenteral glutamine peptide supplements on muscle glutamine loss and nitrogen balance after major surgery. Lancet. 1989; 1:231.233

56 Palmer T E, Griffiths R D and Jones C. Effect of parenteral L-glutamine on muscle in the very severely ill. Nutrition. 1990; 12: 310.320

57 Lochs H, Roth E, Gasic S, Hubl W, Morse E L and Adibi S A. Splanchnic, renal, and muscle clearance of alanylglutamine in man and organ fluxes of alanine and glutamine when infused in free and peptide forms. Metabolism. 1990; 39: 833-836

58 Labow B 1, Souba W W and Abcouwer S F. Mechanisms governing the expression of the enzymes of glutamine metabolism-glutaminase and glutamine synthetase. I Nutr. 2001: 131:2407S-2474S 
Effects of glutamine enriched TPN on interorgan amino acid and gut glutathione metabolism 


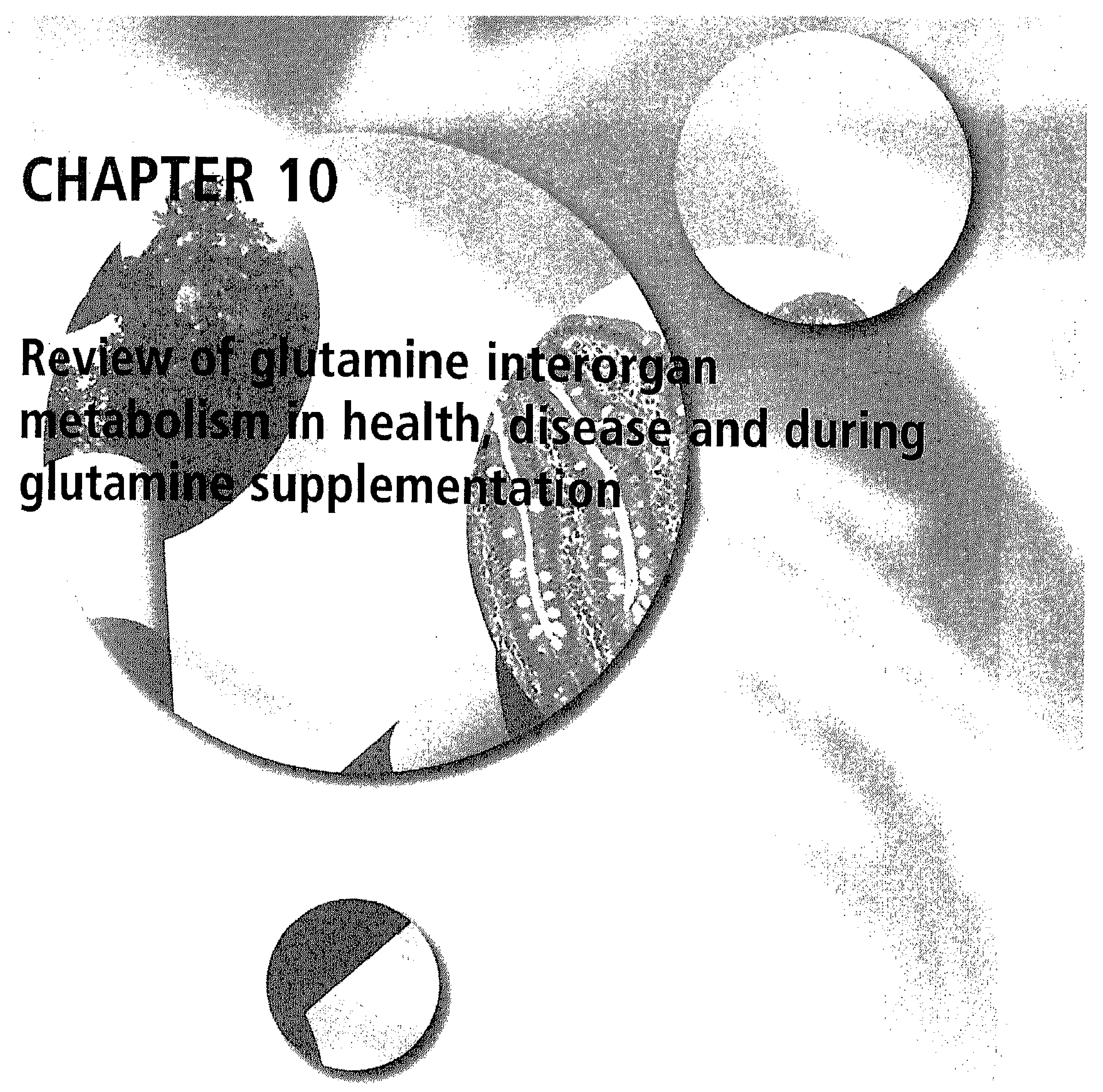




\section{INTRODUCTION}

It has taken approximately a century after its discovery in 1873 as a biologically possibly important molecule !, for glutamine to become the focus of enormous research efforts. After its classification as a non-essential amino acid in the 1930'ies 2 it was not until Eagle et al. reported the importance of glutamine in culture media for cell cultures that glutamine received more attention 3,4 . But only after Windmueller and Spaeth described the essential role of glutamine for the intestine in the 1970'ies, the metabolical role of glutamine in mammals gained the attention it now has. Since then the focus of research has shifted to the clinical applications of glutamine supplementation $5 \cdot 7$.

Historically glutamine has been classified as a non essential amino acid since it can be synthesized de novo in most mammalian tissues, and indeed it was demonstrated that glutamine was not required as a dietary nutrient 2 . However, later this concept has been challenged ${ }^{8}$ and (relative) glutamine deficiency has been thought to occur under certain pathophysiologic conditions ${ }^{9}$. However, this issue is still subject to debate 10.

The concept of glutamine deficiency under certain conditions has formed the rationale for its supplementation. According to Pubmed, at present 345 prospective randomized clinical trials have been performed testing glutamine supplementation either alone or in combination with other nutrients or nutraceuticals. The results of these trials have yielded equivocal results, feeding the discussion whether glutamine is conditionally essential or not. In order to better understand and interpret these data, knowledge of glutamine interorgan metabolism is of paramount importance. Despite all the work that has been done, many gaps remain in our understanding of the metabolism of glutamine. In this manuscript we aim to summarize the available data with regard to glutamine (interorgan) metabolism.

After a general discussion of characteristics of glutamine and general aspects of its metabolism and transport, interorgan glutamine metabolism in healthy organisms will be discussed. Given the significant changes occurring in glutamine metabolism in several pathophysiological states, these will be discussed next. Many of the changes observed in sepsis can also be found in patients in other catabolic states such as after trauma and postoperatively. In general, the changes observed in sepsis occur more rapidly and are more profound compared to those in other situations. For the purpose of this review, we will focus on changes occurring in glutamine metabolism during the aforementioned conditions. Glutamine metabolism is also significantly altered in (advanced) malignant disease. One specific characteristic consists of a net flux of glutamine from host to the tumor. The size and type of tumor may significantly determine glutamine kinetics. In this review cancer will not be discussed. For a review of this topic the reader is referred to a recent publication by Medina " 1 . Finally, the effects of exogenous administration of glutamine on its endogenous (interorgan) metabolism will be reviewed.

\section{Glutamine metabolism}

a. Chemical and physiological characteristics of glutamine

Glutamine consists of a 5 carbon skeleton and differs from glutamate only in the substitution of a carboxylate for an amide group (see reaction scheme in next section). Physicochemical properties of glutamine have been reviewed by Young and Ajami 12 . 
They conclude that the chemical properties of glutamine make it very suitable for (nontoxic) transport of nitrogen under diverse conditions. However, glutamine is also involved as a major player in interorgan carbon transport 13 . Furthermore, glutamine is an important precursor for gluconeogenesis 13,14 .

Glutamine is present in high concentrations in plasma amounting to $20 \%$ of all amino acids and represents approximately $60 \%$ of the free intracellular amino acid pool in muscle 15. After severe injury the organism releases large amounts of amino acids from muscle, mainly in the form of alanine and glutamine, and this is associated with decreases in intracellular glutamine concentrations 15-20. Finally, the very diverse and crucial metabolic lunctions in which glutamine is involved all point to its central position in amino acid metabolism.

Glutamine itself is required for a number of biochemical reactions, but of even more importance is that it is a precursor for glutamate ${ }^{21}$. Glutamate also plays a central role in amino acid metabolism but its transmembrane transport is limited. Moreover, in contrast to glutamine, glutamate is present only in low quantities in the extracellular environment. Therefore, compared to glutamate, whose actions are primarily intracellulal, glutamine is distinctly more important in interorgan metabolism 22 .

Although the metabolic fate of glutamine depends on the tissue and on the presence or absence of disease, generally four categories can be distinguished for the roles of glutamine: 1) nitrogen transport, 2) maintenance of cellular redox state, 3) intermediary metabolism and 4) provision of energy 23 . Several extensive reviews of the metabolic roles of glutamine have been published previously $9,21,23-25$.

One disputed effect of glutamine concerns its relation with protein synthesis and turnover rates. Rennie et al. hypothesized that intracellular glutamine concentrations regulated protein turnover rates $2 \hbar$. Although an association between glutamine and protein synthesis rates has been demonstrated 27.30, others could not confirm this and its causal relationship is therefore under debate 31.35 . The fact that in severely catabolic patients muscle glutamine concentrations strongly decrease while at the same time muscle protein synthesis rates increase also argue against a direct relation 36,37 .

Glutamine is not only endogenously produced, but can also be derived from dietary intake. However, this is not necessarily true for the fetus and premature infants for whom glutamine may be an essential amino acid 38 . Already in 1970 glutamine was reported to be the quantitatively most important amino acid to be transferred from mother and placenta to the fetus in sheep ${ }^{39}$. If this glutamine supply from the mother is interrupted prematurely, glutamine deficiency may occur especially since premature infants often do not receive enteral nutrition for weeks. Glutamine supplementation to TPN in this group resulted in clinical benefits such as a lower occurrence of nosocomial infections ${ }^{40}$ and less need for mechanical ventilation ${ }^{41}$. Moreover, glutamine metabolism has been studied with stable isotopes in this population as will be discussed later in this text.

\section{b. Glutamine synthetase (GS)}

Since glutamine plays such a central role in diverse essential metabolic pathways, its production, consumption and transport are tightly controlled. Interorgan fluxes orchestrate the complex balance between production and consumption in different organs, but ultimately glutamine metabolism is determined by its production and catabolism. Under 
postabsorptive conditions free glutamine can be derived either from release of previously bound glutamine, e.g. during proteolysis, or synthesized de novo. It has been estimated that $60 \%$ of the glutamine present in plasma is the product of de novo synthesis 13,42 . GS catalyses the cytoplasmic formation of glutamine from glutamate and ammonia:

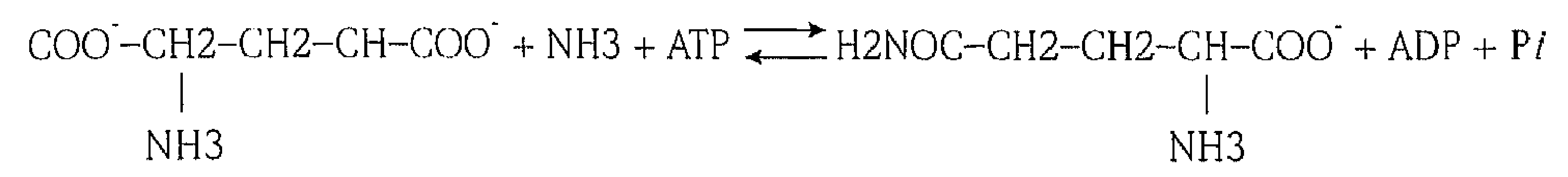

GS is the only enzyme capable of synthesizing glutamine de novo and has been demonstrated in mammalian heart, lungs, spleen, skeletal muscle, liver, brain, intestine, kidney and adipose tissue ${ }^{43-46}$. Congenital mutations in GS have been described and result in virtually absent serum glutamine levels ${ }^{47}$. GS expression is increased after injury, during severe inflammatory stress or physical activity, after glucocorticoid administration and during starvation ${ }^{48.50}$. This occurs particularly in muscle and lung tissues. The activity of GS appear's not to be affected by post translational modifications and, therefore GS expression is a major determining factor in glutamine production rates 51 (for a more detailed biochemical review see 40 ). However, GS protein levels do not always correlate with GS mRNA levels suggesting that it may be subject to posttranscriptional regulation. Since changes in GS protein levels require $6-10$ hours to occur, acute changes in glutamine synthesis must be regulated by other mechanisms than changes in GS synthesis rates. The fact that a vast excess of GS protein is present for any flux rate in vivo further supports this ${ }^{52}$. Regulation of GS expression occurs at two levels: increased transcription due to hormonal effects and changes in (SS breakdown rates with varying glutamine concentrations 51 . Increasing glutamine levels in cultured muscle cells inhibit GS activity 53 . Labow et al. demonstrated that glutamine causes a rapid decline in GS protein levels in a cultured lung cell line due to increased GS breakdown 51 . This dual regulation of glutamine synthesis enables the body to rapidly adjust to fluctuating demands for glutamine during stress or trauma.

\section{c. Glutaminase}

The first step in glutamine catabolism is deamidation resulting in the formation of glutamate. Several cytosolic transamidases can use the amide group released in a variety of metabolic pathways 54 . For the purpose of metabolic control however another pathway appears to be more appropriate: the mitochondrial enzyme GA catalyses the hydrolysis of glutamine to form glutamate and ammonia. The ammonia released can be used to form carbamoyl phosphate or diffuse out of the mitochondria and the cell. Glutamate in turn can be further deaminated to form $\alpha$-ketoglutarate which is metabolized in the citric acid cycle. This degradation pathway prevents formation of excessive amounts of specific metabolites 51.

Glutaminase is a mitochondrial enzyme which is probably located on the outer surface of the inner mitochondrial membrane 55, 56. Two isoforms of glutaminase exist: the "liver-type" glutaminase which only is expressed in periportal hepatocytes, and the "kidneytype" which is distributed widely in non-hepatic tissues. It is particularly abundant in kidney, brain, intestine and in rapidly dividing cells of the immune system. Furthermore, from the "kidney-type" GA 3 isoforms have been identified each with specific tissue 
distribution ${ }^{51}$. Both glutaminases are activated by phosphate. Although both isoenzymes have a high degree of identity in amino acid sequences, they are products of different genes and have different structural and kinetic properties $5 \%$. For example, whereas the kidneytype is inhibited by glutamate (one of its products), in the liver a positive feedback mechanism exists whereby glutaminase is activated by ammonia ${ }^{50}$.

GA can be activated by glucocorticoids and glucagon but also glutamine has been suggested to enhance glutaminase activity 58.59 . Starvation, glucocorticoids, experimental diabetes and high protein diets lead to increased amino acid breakdown and have been reported to increase GA expression ${ }^{60.63}$, presumably to support gluconeogenesis and/or nitrogen disposal through the urea cycle ${ }^{51}$. On the other hand, these effects may be tissue specific as severely catabolic situations such as sepsis and advanced malignancy have been reported to decrease GA activity in intestinal mucosa 58 . Possibly, a decreased glutamine transport 58 or changed membrane potentials result in lowered intracellular glutamine concentrations, which in turn negatively affect GA activity.

\section{d. Importance of glutamine interorgan metabolism}

Glutamine is abundantly present as a free amino acid in plasma and in intraccllular fluids, particularly in muscle. The total free glutamine pool in the plasma compartment amounts to approximately $1800 \mu \mathrm{mol}$ in a healthy adult. The total extracellular free glutamine pool is estimated to be $110 \mu \mathrm{mol} / \mathrm{kg}$ body weight 22 . The free intracellular glutamine pool approximates $5200 \mu \mathrm{mol} / \mathrm{kg}$ body weight 22 and the largest part resides in skeletal muscle. But this is still small in comparison to the amount of protein bound glutamine which is approximately $296 \mu \mathrm{mol} / \mathrm{g}$ body protein ${ }^{\circ}$. Assuming that the body of a $70 \mathrm{~kg}$ man contains approximately $12 \mathrm{~kg}$ protein, this would account for $3552 \mathrm{mmol}$ glutamine.

Despite these large intracellular quantities glutamine plays a less important role in intermediary metabolism than its principal breakdown products glutamate, and other breakdown products. However, its importance resides in the fact that it is quantitatively the major non-toxic nitrogen and carbon carrier between organs. Approximatcly one third of all nitrogen derived from protein metabolism in plasma is in the form of glutamine 23 . Fia et al. reported glutamine to carry $82 \%$ of amino acid nitrogen release from muscle $1 \%$. With regard to carbon derived from proteolysis, glutamine carries more than twice the amount of carbon compared to alanine in the plasma compartment 13.

Glutamine concentrations vary greatly between plasma and various tissues. This compartmentalization requires an adapted transport system to maintain these gradients. Specific molecules have been identified in cell membranes which regulate transport of glutamine and other amino acids. In addition, glutamine compartmentalization also exists within the cell: for example glutamine concentrations in liver cell cytoplasm are significantly lower than in the mitochondria 60 . Glutamine concentration differences between different cell organelles are also regulated by transporters 67 . In this review we will focus on glutamine transporters in the cell membrane.

\section{e. Glutamine transport}

The characteristics of amino acid including glutamine transporters have recently been reviewed 68,69 . In general, antiport rather than a combination of uniporters and co- 
transporters appear to be the prevalent mode of amino acid transport. Glutamine, however, is transported mainly by 3 different transport systems with different characteristics. Although a $4^{\text {th }}$ transport system, i.e. system $\mathrm{L}$, also recognizes small neutral amino acid such as glutamine, its activity contributes relatively little to glutamine transport 70 . It will therefore not be discussed further.

System A transport (a $\mathrm{Na}^{+}$co-transporter), accumulates glutamine up to 110 -fold and appears to play a role in maintenance of an elevated intracellular glutamine concentration. This transporter uses the transmembrane $\mathrm{Na}^{+}$electrochemical gradient maintained by the $\mathrm{Na}^{+} / \mathrm{K}^{+}$- ATP-ase. System A transporters have been identified in liver, brain, heart, kidney, colon, small intestine, lung, muscle, spleen, stomach, adipocytes, testis and placenta 69,71 .

System $\mathrm{N}$ transport concerns a $\mathrm{H}^{+}$antiporter which can readily reverse the transport direction under changing conditions, such as changes in $\mathrm{pH}$, but also transmembrane glutamine gradients can affect the direction of transport 72 as hypothesized in 1986 by Rennie ${ }^{20}$. Therefore, the presence of system $\mathrm{N}$ probably determines whether a cell or tissue can release glutamine or not. This transporter is expressed most strongly in liver and in lower amounts also in skeletal muscle, kidney, heart and brain ${ }^{09}$.

Finally, the antiporter ASCT2 uses a prevalent amino acid such as glutamine in exchange for uptake of small neutral amino acids in for example the small intestine. This transporter is expressed in placenta, lung, kidney, pancreas, skeletal muscle, adipose tissue, intestinal epithelia and astrocytes but not in neurons $69,73,74$.

Glutamine transport across the plasma membrane can be affected by cytokines such as TNF- $x$ and IL-1, but the result appears to be tissue or cell specific $75 \cdot 77$. Moreover, hormones (e.g. insulin) and the electrochemical membrane gradient exert controlling functions over glutamine transport $20,78,74$. This phenomenon may be one of the fundaments of the regulation of changes in interorgan glutamine metabolism.

The mechanisms underlying the release of glutamine from the free glutamine pool in the muscle in vivo have not been clearly defined yet. Plasma glutamine concentrations have been suggested as a trigger for "pulling" glutamine out of muscle, although this does not explain excessive glutamine release from muscle in acute acidosis where plasma glutamine levels are elevated ${ }^{52}$. Another argument against this hypothesis has been provided by results from a study by Darmaun et al. ${ }^{80}$. They treated healthy humans with phenylbutyrate which is readily converted to phenylacetate. This in turn reacts with glutamine to yield phenylacetylglutamine which is excreted as such in the urine. This "drainage" of glutamine resulted in decreased plasma glutamine levels with approximately $26 \%$, but no changes in glutamine appearance rates or de novo synthesis rates were detected.

Alternatively, changes in transmembrane electrical potential have been hypothesized to be involved in the regulation of glutamine transport 52 (Chapter 6 , this thesis). This topic will be discussed more extensively later.

\section{Measurement of interorgan metabolism: principles and significance}

In contrast to plasma or tissue glutamine concentrations which are static parameters that provide only limited information with regard to production rates, the flux or appearance rate, i.e. the amount of glutamine released into plasma per time, is the parameter of interest when studying interorgan metabolism. Glutamine is produced predominantly by muscle tissue, but 
also by brain ${ }^{81}$, subcutaneous tissue and the lung under catabolic conditions, whereas enterocytes, the immune system and the kidney are well known consumers of glutamine. The liver can secrete as well as take up glutamine depending on the metabolic conditions.

The measurement of glutamine interorgan transport, however, becomes complicated as virtually all tissues can produce as well as consume glutamine at the same time. Because in the hypothesis of glutamine as a conditionally essential amino acid it is assumed that the production of e.g. muscle falls short for big consumers such as the gut, liver and other parts of the immune system, the net rate at which glutamine is secreted (and taken up under steady state conditions) into the plasma by all individual organs and tissues becomes the parameter of interest. This endogenous production rate is also called the glutamine rate of appearance.

This rate of appearance is measured by analysis of the dilution of an known amount of glutamine tracer infused. As the enrichment of plasma glutamine is measured the rate of appearance into the plasma compartment is calculated, which is different from the actual rate of appearance at the cellular level. The production rate of glutamine by an organ represents all glutamine that is released from that organ into the plasma. Simultaneously glutamine is taken up (consumed) by that organ and the difference betwcen the two is the net flux which is measured by the (AV)-balance technique. After a brief introduction of these methods the limitations and pitfalls of these techniques will be discussed.

\section{a. AV balances}

The AV-balance technique is based on the concentration difference of glutamine across an organ (i.e. arterial versus venous concentrations) which is multiplied by the flow of blond across that organ. This flow can be measured with indicator dilution techniques, plethysmography, dye clearance or thermodilution methods or more directly with sophisticated Doppler techniques both measuring vessel diameters and flow velocities, which allows together calculation of flow. In human experiments most studies have used dye dilution to measure glutamine production rates across an extremity.

Obviously, measurement of AV-balances in man is restricted by the accessibility of the respective organs. Much experience has been obtained in measurement of glutamine production across the forearm and the leg, but contributions of e.g. the intestine, the liver; the spleen or the lung have been much less well determined in humans although their importance in glutamine metabolism has been well established in animal models. Therefore the technique of AV-balances cannot provide more than a partial picture of the whole body glutamine kinetics. Furthermore, these catheterization studies usually assume that the blood samples taken specifically represent the tissue of interest. Thus venous blood derived from forearm or leg catheterization studies are assumed to represent venous drainage from skeletal muscle, but they also derive blood from skin, adipose tissue and bone. Depending on the method of flow measurement, specific assumptions are made which may not necessarily be true ${ }^{82}$. Moreover, as flow increases AV-differences will become smaller resulting in higher variations of the final results 83 . But also inaccuracies in flow measurements can result in differing results. Finally, a tissue measured may not be representative for similar tissues elsewhere in the body.

Concluding, results of AV-balance techniques are valuable to determine semiquantitative changes taking place in the living organism. However, small but physiologically important 
interorgan fluxes may remain undetected. The results only reflect the specific organs measured, and only provide the net result of uptake and release across the organ of interest, but do not provide an estimate of actual production and uptake.

\section{b. Tracer models}

The isotope dilution technique basically measures the dilution of the tracer (i.e. the tagged molecule of interest) in the sampled (usually plasma) compartment. Therefore this technique gives an estimation of the total amount of glutamine entering the plasma compartment per time. Under (metabolically) steady state conditions this figure also equals the amount of glutamine simultaneously taken up from the plasma compartment. Thus, in contrast to results obtained by AV-balance techniques, which provide an estimate of the net release of glutamine by the organ studied, isotope dilution techniques provide results reflecting the sum of the net contributions of all organs to glutamine kinetics. Most organs can take up and release glutamine simultaneously. However, except for the liver, the organs usually show either' net uptake or net release of glutamine.

Ideally, isotopic steady state exists before samples are taken to determine the enrichments of the tracer infused. For many tracers this is a matter of a few (up to 6) hours before this is achieved. However, glutamine has a very large and relatively slowly mixing
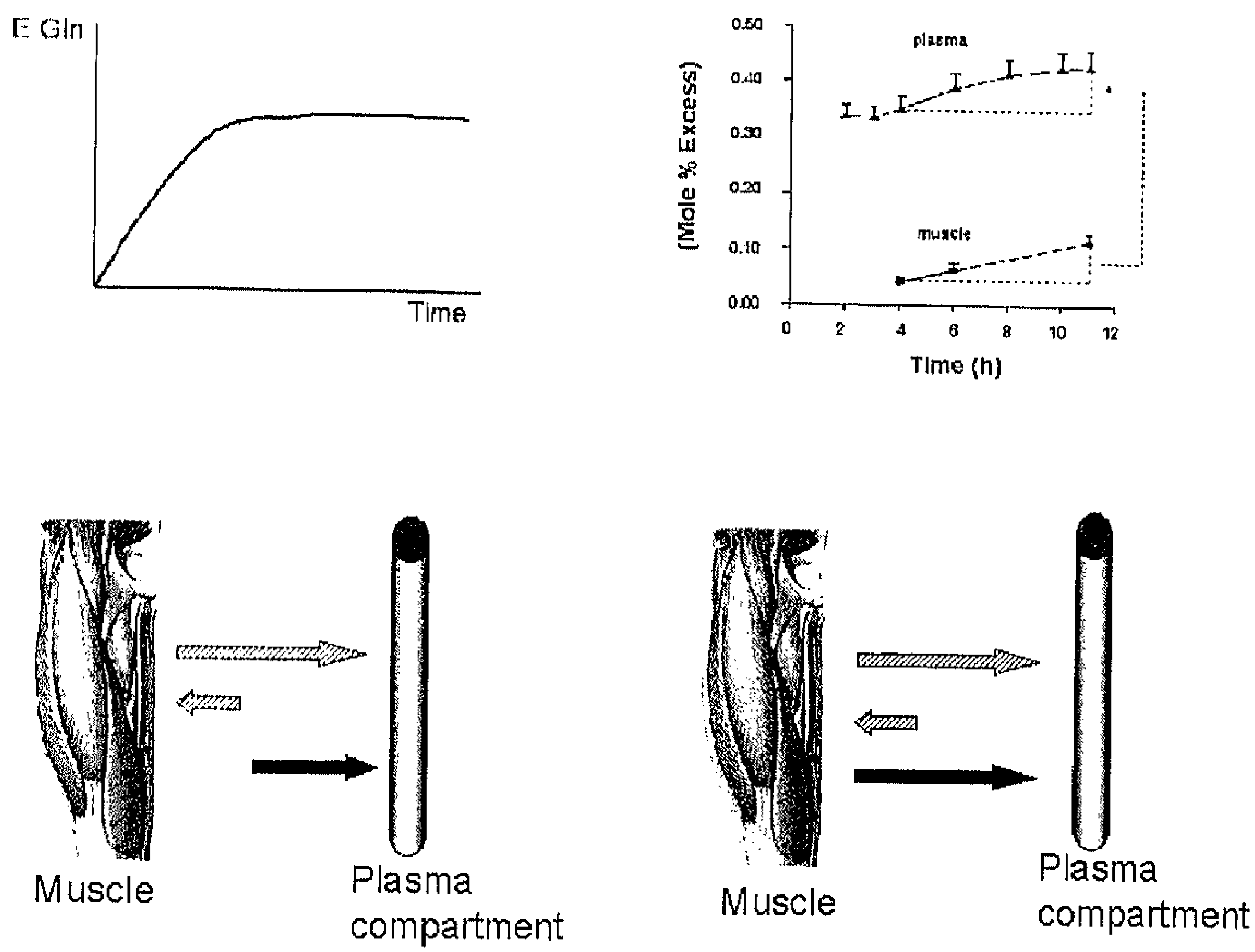

Figure 1 Physiological significance of glutamine kinetics measured in steady state conditions (left part) or in non steady state conditions (right part). In the upper part plasma glutamine tracer enrichments are shown indicating steady state and absence of steady state respectively. The open arrows indicate total glutamine outward and inward transport respectively, whereas the black arrows indicate the actually measured glutamine transport rate in either isotopic steady state or non-steady state conditions. 
intracellular pool, resulting in a long time period before steady state is achieved $22,42,84$. We estimated that it would take 24.36 hours before isotopic steady state is achieved after start of a primed constant infusion ${ }^{84}$. This leads consequently to other problems such as loss of metabolic steady state, tracer recycling etc. However, most studies investigating glutamine kinetics with isotopes have used short infusion times. If plasma enrichments early in the course of infusion are used for calculations, isotope dilution approaches the total outward flow of glutamine into the plasma compartment (thus not taking into account the simultaneously occurring uptake of the specific organs) whereas in isotopic steady state the net glutamine flux, i.e. the total outward flow minus the total uptake, is measured. This difference is displayed graphically in figure 1 . The definitions of rate of appearance and flux will be discussed in part II. Beside these methodological difficulties, measurement of isotopes requires the availability of a mass spectrometer, specifically trained analytical personnel and substantial resources. Thus, although isotopic dilution techniques can help us determine glutamine kinetics on the whole body level, its technique for measurement of glutamine kinetics has several important pitfalls and is expensive.

By combining results of AV-balance techniques and isotope dilution it is possible to gain substantial insight in changes taking place in glutamine metabolism under different conditions. A 2-pool model has been described by Barrett and Gelfand in 198785 (see figure 3 of Chapter 1, this thesis). Using this model, information can be acquired regarding glutamine consumption and production rates by the organ of interest. However, this 2-pool model does not take into account the recycling of amino acids within the organ of interest, resulting in underestimations of both protein synthesis and degradation rates. To confront this problem, Biolo et al. developed a 3-pool model in which arterial and venous blood samples in combination with biopsies from the organ of interest theoretically provide enough information to calculate protein (and amino acid) kinetics within the cell and across the cell membrane in more detail (see figure 3 of Chapter 1, this thesis) ${ }^{80}$. However, this method has some important caveats. The increased invasiveness of these measurcments prevents repeated sampling resulting in limited precision of the intracellular enrichment and concentration measurements. A meticulous preparation of the biopsies, such as removing blood from the samples and removing non-relevant tissues from the samples (e.g. connective tissue in muscle biopsies) is even more important when using these models. And last but not least, concentration and tracer enrichments must be constant in all the pools (both in blood and in the tissues) during the study, i.e. tracer steady state is required ${ }^{87}$.

In summary, the data provided by calculating whole body glutamine kinetics only give an cumulative picture of glutamine interorgan metabolism. If an increased glutamine production of one organ is compensated by lower production or even uptake by another organ, this might not be reflected in the whole body flux. Evidence exists to illustrate this point 88,89 . However, isotopic studies can be useful to determine whether a possible shortage in endogenous glutamine supply exists during disease by comparing glutamine kinetics under differing pathophysiological circumstances.

\section{c. Animal models versus human studies}

Initially, most experimental studies involved rats as study objects. This choice was fortunate in the sense that rodents are very sensitive to glutamine deficiencies and probably more so 
than humans. Among other factors, this might explain why the impressive results on morbidity and mortality obtained in rodent models in which glutamine was given, could not be replicated so easily in human patient studies. This fact does not negate the enormous progress in our understanding of glutamine metabolism which has been obtained by using mouse and rat experiments, but one should be careful to extrapolate the results obtained to the human situation. Another example involves handling of glutamine by the portal drained viscera. Whereas the intestinal tissue of humans, dogs, pigs and rats consumes glutamine, other species such as the guinea-pig and chicken show net glutamine output across the gut "0. Moreover, in sheep most glutamine uptake by the PDV was by non intestinal tissues. In addition the fate of glutamine taken up by the PDV differed from data obtained in other models ${ }^{91}$. Therefore, findings obtained in a certain animal model should not be extrapolated too much. Of course comparability with human metabolism differs from animal to animal.

In this review human data will be used whenever available, but otherwise material from mammalian animal experiments will be used. Specifically, AV-balances across splanchnic organs and the kidneys require such invasive procedures, that most available data are derived from animal experiments. Data obtained in animal models closely resembling human glutamine metabolism such as dogs and pigs will be used preferentially. Differences in glutamine metabolism related to e.g. enzyme activities etc. will be indicated whenever appropriate.

\section{Glutamine metabolism in healthy organisms \\ a. Whole body data \\ i. Significance of (plasma) concentrations}

Glutamine concentrations in plasma need to be measured in arterial blood, since venous concentrations are more variable depending on the location of sampling used. However, in order to minimise invasive procedures, sampling arterialised venous blood by using external heating of e.g. hand veins is frequently used as an alternative. Normal concentrations vary around $600 \mu \mathrm{mol} / \mathrm{L}$. Insulin, epinephrine and glucagon all lower glutamine concentrations in healthy fasted human subjects ${ }^{92}$. Exhaustive and prolonged exercise similarly results in temporary reductions in glutamine plasma levels in humans ${ }^{93}$. Finally, prolonged starvation also results in decreased glutamine plasma levels ${ }^{94}$. Despite the effects of insulin on glutamine plasma concentrations, glutamine Ra and glutamine de novo synthesis rates in vivo appear not to be affected by insulin ${ }^{95}$. Therefore it is important to distinguish changes in concentration from changes in fluxes. Frequently changes in arterial concentrations are explained by (temporal) imbalances in Ra and Rd. Although it is true that plasma concentrations are the net result of $\mathrm{Ra}$ and $\mathrm{Rd}{ }^{96}$, the reverse is not true, i.e. the plasma glutamine concentration does not indicate the level of either the Ra or Rd of glutamine. Since the plasma pool only contains a very limited amount of glutamine (approximately $1800 \mu \mathrm{mol}$ in a $70 \mathrm{~kg}$ individual) compared to the intracellular stores, but also in comparison to the fluxes of glutamine, imbalances between Ra and Rd can only exist shortly prior to complete exhaustion of the plasma pool. Assuming for example a glutamine rate of appearance of $5.0 \mu \mathrm{mol} / \mathrm{kg} / \mathrm{min}$, an individual would exhaust the plasma pool in approximately 5 minutes assuming that uptake rates remained constant and no glutamine would enter the plasma pool within this time. In a less extreme scenario, a decrease in this glutamine Ra of $5.0 \mu \mathrm{mol} / \mathrm{kg} / \mathrm{min}$ with $10 \%$ with a Rd that remains constant, would result 
in a lowering of plasma glutamine concentrations $(600 \mu \mathrm{mol} / \mathrm{L})$ with $20 \%$ within approximately 10 minutes. Therefore, decreases in glutamine plasma concentrations, although the result of brief and/or small imbalances between Ra and Rd, do not necessarily indicate altered flux rates nor possible glutamine deficiency, but rather reflect an altered equilibrium between the different glutamine pools. This is illustrated by data from Meyer et al. who studied the effects of insulin infusion on glutamine kinetics ${ }^{17}$. They found decreases in glutamine plasma concentrations despite increases in glutamine Ra and Rd. The Rd, however was during a short time somewhat higher than the Ra. Hundred and fifty minutes after starting the insulin infusion, glutamine $\mathrm{Ra}$ and $\mathrm{Rd}$ were not different anymore.

The complex regulation of plasma glutamine concentrations has not been fully elucidated yet. Hormonal changes affecting glutaminase or glutamine synthetase expression may play a role in explaining changes in glutamine concentrations. However, these changes usually require hours or days to take place. A more obvious regulating mechanism resides at the level of glutamine transport across the cell membrane. Here the different transporters play an important role. But also changes in their quantities and activities require some time. Although the fall in glutamine plasma concentrations is usually much more limited compared to the decrease seen in the intracellular muscle pool ${ }^{98}$, it has been theorized that this decrease could be somehow sensed and subsequently lead to a pull (i.e. enhanced exportl of glutamine out of the muscle pool. However, large changes in transport can occur before significant decreases in plasma glutamine concentrations occur. Another attractive hypothesis is that in times of e.g. severe injury or inflammatory stress, glutamine is released because, due to decreases in membrane potential during severe disease ${ }^{99}$, the cells are unable to retain glutamine intracellularly. Endotoxinaemia and inflammatory mediators have been shown to decrease the resting membrane potential 100.101. This is the result of marked increases in intracellular sodium and chloride concentrations and a decrease in intracellular potassium ${ }^{19}$. In rat liver cells the same phenomena has been observed after exposure to oxidative stress and has been linked to cell shrinkage ${ }^{102}$. Because transport of glutamine into the cell depends on this membrane potential, this process can lead to a shortlived net efflux of glutamine out of the cell into the plasma compartment, leading to a new equilibrium between intra- and extracellular glutamine concentration. This phenomenon could explain the sudden and unalterable decrease of intracellular glutamine seen during conditions of severe inflammatory stress (Chapter 6, this thesis). Moreover, this also provides an explanation for the obvious failure of glutamine supplementation to restore intracellular glutamine concentrations to normal levels in critically ill patients 103.105 in contrast to e.g. patients after elective surgery 100.108 .

We therefore propose that changes in arterial glutamine concentrations do not result from nor are a sign of "exhaustion" of the (muscle) glutamine supply, but rather result from changes in membrane properties which lead in turn to changes in transmembrane gradients of glutamine. The linkage of glutamine transport to sodium and proton gradients (system $\mathrm{A}$ and $\mathrm{N}$, see part I of this discussion), links glutamine gradients to the transmembrane electrochemical gradient. Therefore, changes in intracellular glutamine concentrations do not only result from changes in transporter activities, but also from opening of specific ion channels in the cell membrane, or changes in $\mathrm{pH}$ or in sodium transport which are the result from direct effects of e.g. toxins and cytokines on the cell membrane. 


\section{ii. fluxes: interpretation and methodological issues}

Having established, that the glutamine rate of appearance rather than the glutamine plasma concentration is of value in determination of a possible glutamine deficiency, it is first necessary to define the terms used, since these can cause some confusion. According to Wolfe the rate of appearance $(\mathrm{Ra})$ refers to the total rate of appearance of the tracee 109. The source of glutamine appearance lies within the cell. Glutamine appears intracellularly as a result of de novo synthesis, protein breakdown or uptake from the blood. This glutamine may, however, already be degraded or used for synthesis before it is exported to the plasma. The Ra of glutamine that is measured in plasma (as is the case in the studies described in this thesis) is the net result of these intracellular processes, and therefore does not reflect the intracellular appearance rate. Since intracellular appearance rates are difficult to measure, most data concern glutamine appearance rates in the plasma compartment. Within the current context Ra concerns the appearance into plasma, unless otherwise specified. In a single pool model the Ra is determined by the endogenous production and exogenous infusion. In multiple pool models obviously the Ra needs to be defined in more detail. The rate of disappearance $(\mathrm{Rd})$ applies to the rate of tracee which is irreversibly lost from the plasma pool (or a specified other pool in multiple pool models) through tissue uptake or loss with e.g. the urine. The calculations generally used to determine Ra and Rd of glutamine require isotopic steady state ${ }^{109}$. In metabolically steady state conditions the Ra equals the Rd. Although the terms flux and turnover rate can be confusing at times, in the context of this manuscript they will be used interchangeably with Ra. The term net flux is reserved for the net glutamine production or consumption across an organ (AV- balance $\mathrm{x}$ flow).

In various studies whole body glutamine fluxes in healthy organisms have been measured. Whole body glutamine fluxes in rodents vary around $22-25 \mu \mathrm{mol} / \mathrm{kg} / \mathrm{min}$ in healthy rats 88,110 . In postabsorptive dogs whole body glutamine fluxes are $\sim 10$ $\mu \mathrm{mol} / \mathrm{kg} / \mathrm{min}$ (i.e. approximately double the rate encountered in humans) although a significant decrease in flux was observed with longer duration of the isotopic infusion 111 . This phenomenon will be discussed subsequently. Furthermore, neither exercise nor prolonged starvation significantly changed glutamine $\mathrm{Ra} 111$. In sheep glutamine turnover rates of $2.9 \mu \mathrm{mol} / \mathrm{kg} / \mathrm{min}$ and $3.5 \cdot 4.5 \mu \mathrm{mol} / \mathrm{kg} / \mathrm{min}$ have been reported in the postabsorptive and the fed state respectively $91,112,113$. In postabsorptive pigs we found a glutamine Ra of $5.8 \pm 0.5 \mu \mathrm{mol} / \mathrm{kg} / \mathrm{min}$ (Ch 3, this thesis) which is in the same order of magnitude compared to the values found in humans.

A landmark paper on whole body glutamine fluxes in healthy human subjects was written by Darmaun et al. 22 Using two isotopically labelled glutamine tracers (L- $[2-15 \mathrm{~N}]$ glutamine and L-[5-15N]-glutamine) during a 4 hour unprimed infusion protocol, they obtained glutamine appearance rates of $283 \pm 31$ and $348 \pm 33 \mu \mathrm{mol} \cdot \mathrm{kg}^{-1} \cdot \mathrm{h}^{-1}$ respectively. This difference was attributed to recycling of glutamine through transamination. Glutamine whole body flux rates have been measured repeatedly in healthy volunteers under postabsorptive conditions but varying in the type of label used and in infusion protocols. Results of measurements of whole body glutamine turnover in healthy humans are summarized in table 1. 
Table 1 Glutamine rate of appearance in healthy human subjects in mean $\pm S E M(\mu \mathrm{mol} / \mathrm{kg} / \mathrm{min})$. Results are shown per glutamine tracer used

\begin{tabular}{|c|c|c|c|c|c|c|c|}
\hline $2-15 \mathrm{~N}$ & $5.15 \mathrm{~N}$ & $1.13 \mathrm{C}$ & $3.4-{ }^{13} \mathrm{C} 2$ & $1.2^{13} \mathrm{C} 2$ & U.14C & $3.4^{3} \mathrm{H}$ & Reference \\
\hline \multirow[t]{2}{*}{$4.7 \pm 0.51$} & $5.8 \pm 0.55$ & & & & & & 22 \\
\hline & & & & & $5.76 \pm 0.26$ & & 13 \\
\hline \multirow[t]{2}{*}{$4.67 \pm 0.39$} & & & & & $5.72 \pm 0.54$ & $6.14 \pm 0.54$ & 113 \\
\hline & $5.92 \pm 0.40$ & & & & $6.22 \pm 0.31$ & $6.55 \pm 0.40$ & 42 \\
\hline \multirow[t]{4}{*}{$5.7 \pm 0.43$} & & & & & & & 114 \\
\hline & $4.5 \pm 0.3$ & & & & & & 115 \\
\hline & & $5.1 \pm 0.4$ & & & & & 79 \\
\hline & $4.1 \pm 0.3$ & & & & & & 35 \\
\hline \multirow[t]{2}{*}{$5.3 \pm 0.30$} & & & & & & & 116 \\
\hline & & & & & & $4.3 \pm 0.33$ & 42 \\
\hline \multirow[t]{2}{*}{$4.9 \pm 0.22$} & & & & & & & 117 \\
\hline & & & & $5.1 \pm 0.13$ & & & 118 \\
\hline \multirow[t]{3}{*}{$5.41 \pm 0.47$} & & & & & & & 119 \\
\hline & & & & & & $6.11 \pm 0.44$ & 120 \\
\hline & & & $5.2 \pm 0.23$ & & & & 94 \\
\hline $5.20 \pm 0.22$ & & & & & & & 121 * \\
\hline \multirow[t]{4}{*}{$4.15 \pm 0.33$} & & & & & & & $121 \dagger$ \\
\hline & $4.3 \pm 0.22$ & & & & & & $83 \#$ \\
\hline & $3.1 \pm 0.23$ & $3.4 \pm 0.30$ & & & & & $83 \$$ \\
\hline & $4.81 \pm 0.23$ & & & & & & 122 \\
\hline \multirow[t]{6}{*}{$5.2 \pm 0.25$} & & & & & & & 123 \\
\hline & $9.0 \pm 1.7$ & & & & & & 124 \\
\hline & & & & & $5.9 \pm 0.3$ & & 96 \\
\hline & & & & & 4.7 & & 125 \\
\hline & $12.6 \pm 2.5$ & & & & & & 126 \\
\hline & & & & & $6.3 \pm 0.5$ & & 127 \\
\hline \multirow[t]{2}{*}{$7.0 \pm 0.5$} & & & & & & & 128 \\
\hline & 7.3 & & & & & & 129 \\
\hline
\end{tabular}

* in healthy controls $<35$ years

tin healthy controls $>60$

\# after 6 hour isotope infusion

$\$$ after 11 hour isotope infusion

Although most studies indicate that the Ra of glutamine amounts to around 4-5 $\mu \mathrm{mol} / \mathrm{kg} / \mathrm{min}$, the values obtained in different studies vary up to a factor 3 . Several factors can account for the differences found. Firstly, data in healthy newborn or premature infants show their glutamine rate of appearance to be a factor 2-3 times higher compared to healthy adults. This can be explained by enhanced protein turnover and increased de novo synthesis 
rates 125 . Secondly, a major methodological problem is caused by the fact that within the timeframe of infusion used in all the studies mentioned above, isotopic steady state conditions are not reached. This was demonstrated unequivocally in our study in gastrointestinal cancer patients where we used an infusion period of 11 hours using 2 different isotopic labels ${ }^{84}$. During this period plasma enrichments of both glutamine tracers continued to rise leading to significantly lower rates of appearance. We have extended these measurements in healthy adults for up to 24 hours and again found absence of isotopic steady state in plasma (unpublished observations). Possible explanations are recycling of the nitrogen tracer, inadequate priming dosages, absence of metabolic steady state conditions during the prolonged study or the absence of complete equilibration of the tracer during the infusion period. By using a $1{ }^{13} \mathrm{C}$ glutamine tracer, which does not recycle since the label is irreversibly lost after oxidation because the $1-C$ carbon of glutamate is predominantly oxidized 131 instead of being reincorporated into glutamine, we were able to determine that approximately $15 \%$ of the rise in $15 \mathrm{~N}$ enrichment between 2 and 11 hours could be explained by recycling of the $15 \mathrm{~N}$ tracer. Changes in protein breakdown rates resulting from prolonged starvation could explain part of the decreases in glutamine appearance rates. In our paper mentioned above, we demonstrated that indeed whole body protein breakdown rates measured with labelled phenylalanine decreased resulting in potentially $16.21 \%$ decline in glutamine $\mathrm{Ra}$ assuming that no changes in glutamine de novo synthesis rates occurred. Inadequate priming doses have been suggested as the main reason for not obtaining steady state in short infusion periods 35,132 . However, it can be calculated that even with a higher (3-hour) priming dose no sufficient labelling of the intracellular glutamine pool in muscle can be achieved during short infusion protocols ${ }^{87}$. The principal reason for the continued rise in plasma glutamine enrichments was the incomplete equilibration of the glutamine tracer with the intracellular muscle pool. During the course of the infusion a linear rise in intracellular muscle glutamine enrichment was observed, explaining the continuing rise in plasma enrichments. Moreover, calculations of the distribution volume of infused glutamine tracers indicated that full equilibration with intracellular glutamine pools were not reached 22, 42, 114. Darmaun et al. calculated that the tracer miscible pool represented only $\approx 2 \%$ of the total whole body free glutamine pool size 22,129 . Increases in muscle glutamine enrichments of glutamine have also been established in primed infusion protocols between 120 and 300 minutes after the start of the tracer infusion 35 .

Not only are these considerations of importance to judge the validity of the calculations made, but more importantly they have major bearing on the interpretation of the physiological meaning of the tracer data ${ }^{133}$. Most tissues, including muscle, not only release glutamine but also take up glutamine at the same time. Assuming that no tracer at all would enter the intracellular compartments, dilution of tracer in a steady state situation would reflect the total amount of glutamine released into the plasma compartment by all organs. Thus, glutamine uptake by the tissues would not be reflected by this figure. Extrapolation of the enrichment data back to time $=0$ would yield the glutamine enrichment to be used for these calculations. Alternatively, enrichment data obtained shortly after start of the infusion period (e.g. after 2 hours) when a pseudo steady state has been reached can be used for these calculations. However, if glutamine is indeed conditionally essential, i.e. under certain pathophysiological conditions its production by muscle and lung becomes 
insufficient to meet the requirements of other organs such as liver, immune system and gut, then not the total glutamine but the net glutamine appearance rate is of relevance. The net glutamine appearance rate reflects the net balance of glutamine across the organs responsible for its release in the plasma compartment. This figure would be obtained with enrichments obtained after isotopic steady state has been obtained. Unfortunately, so far no experiments have been published where this has been achieved. Therefore, whole body glutamine fluxes measured with tracers during short periods of time significantly overestimate true glutamine Ra. The exact magnitude of this error is not exactly known, but might be $20 \%$ or more. Furthermore, the effects of different pathophysiologic conditions on this phenomenon are not clear. In non-steady state conditions, other equations have been proposed e.g. by Chiasson et al. ${ }^{134}$. But these calculations also make assumptions which are not always valid e.g. in the calculation of the relative importance of glutamine for gluconeogenesis ${ }^{92}$, leading to potentially substantial deviations. Besides these methodological considerations, other factors such as age and BMI may also affect glutamine turnover rates 122 .

In conclusion, published glutamine appearance rates in plasma reflect glutamine interorgan transport rates rather than whole body (net) glutamine production rates $22,12,84$.

\section{iii. Source of circulating glutamine}

The largest part (60-85\%) of circulating glutamine is derived from de novo synthesis $13,42$. $80,95,123,135$. However, using lower values for glutamine content of muscle protein, Kuhn et al. calculated the contribution of proteolysis to glutamine Ra to be $-13 \%$ and de novo synthesis $\sim 87 \%$ in healthy humans ${ }^{04}$. This latter figure was also obtained in (premature) infants (13-15\% derived from proteolysis) using the same values for glutamine content of muscle protein 125,127 . With regard to the site of glutamine synthesis it is important to realise that almost all organs contribute to both synthesis and release into the plasma compartment as well as simultaneous uptake and disposal. For example, in a pig model we estimated that the portal drained viscera, known as important glutamine consumers, produce glutamine at a rate of $892 \mathrm{nmol} / \mathrm{kg} / \mathrm{min}$, while simultaneously glutamine disposal amounted to $3183 \mathrm{nmol} / \mathrm{kg} / \mathrm{min}$ (Ch 3 , this thesis). Only muscle, lung, brain, liver and possibly adipose tissue can be net glutamine providers, i.e. they release more glutamine than is taken up. When considering only the net contribution of the different organs, muscle is the predominant source for glutamine synthesis presumably accounting for 50 $75 \%$ of plasma glutamine $13,35,123$. These results are, however, probably overestimated since glutamine tracer calculations were performed using a 2- and 3-pool model in the probable absence of isotopic steady state as discussed previously. The main source of nitrogen needed for glutamine synthesis in muscle is the catabolism of branched chain amino acids and ammonia 117, 136, 137. Tricarboxylic acid cycle intermediates are probably the main precursors of the carbon skeleton for glutamine synthesis. Direct conversion of glucose only provides a limited amount of the carbon skeleton (5-13\%) 95, 138 .

In fed conditions, glutamine also enters the portal circulation from the gut. It is estimated that $50-75 \%$ of glutamine administered enterally is extracted by the splanchnic bed in healthy humans during the first pass $42,118,119,139$, of which a large part is oxidized within the splanchnic bed 42 . The extraction rates decreased with increasing amounts of glutamine being delivered. Administration of enteral or parenteral nutrition containing no 
or small amounts of glutamine to premature or newborn infants resulted in decreased (8$15 \%$ ) glutamine fluxes 125,127 .

\section{iv. Destiny of circulating glutamine}

Glutamine disappearance from plasma can be explained for $40-65 \%$ by oxidation $13,80,123$, 140 , for $10-20 \%$ by gluconeogenesis $13,123,140$, and most of the remainder to protein synthesis 140 or biosynthesis of other macromolecules. Major sites of uptake are skeletal muscle $(\sim 50 \%) 13,123$, the gastrointestinal tract $(-20 \%) 58,141$, the kidney $(\sim 15 \%) 126$ and liver $(5-10 \%)$ 142.144. The fate of glutamine administered (par)enterally is described in the $3^{\text {rd }}$ section of this chapter dealing with the effects of glutamine supplementation.

\section{b. Muscle}

i. Concentration of glutamine, GS, GA, transporters

Muscle contains by far the largest glutamine pool in the body and is responsible for a major part of glutamine production in glutamine interorgan metabolism both in basal conditions and after injury or inflammatory stress. Free muscle glutamine concentrations vary depending on species ${ }^{14}$ and possibly on the specific muscle from which a biopsy is taken. Data in healthy postabsorptive humans show free glutamine concentrations of $15-20$ $\mathrm{mmol} / \mathrm{L}$ intracellular water ${ }^{35}$. Short term starvation in healthy volunteers results in a approximately $30 \%$ decrease of muscle glutamine concentrations 145 .

Although the expression of glutamine synthetase in muscle is not high, due to its large mass muscle is the main site for glutamine production under postabsorptive conditions 19 . Alanine and glutamine make up $60 \%$ of the total amino acid production by muscle tissue although this is far more than their presence in muscle protein 117,140 . This probably reflects the importance of de novo synthesis, whereby the nitrogen is primarily derived from branched chain amino acid metabolism 137. On the other hand, it has been estimated that $25-55 \%$ of glutamine released from muscle is formed de novo, which is considerably lower compared to whole body values 35,147 . In muscle, glutamate needed for glutamine synthesis is derived either from uptake from the plasma compartment, from protein degradation or from the reaction of 2-oxoglutatarate (a citric cycle intermediate) with branched chain amino acids (BCAA's) catalysed by BCAA transaminase. The ammonia needed for glutamine synthesis can be obtained either from the intracellular pool or deamidation of the BCAA's. By determining uptake of a labelled ammonia tracer, Lockwood et al. estimated that $50 \%$ of circulating ammonia was removed by skeletal muscle 148. Glutaminase activities in human skeletal muscle are low and can possibly explained by formation of a less active isoform in this tissue ${ }^{149}$.

Muscle tissue not only releases glutamine but also takes up glutamine in large amounts. Glutamine availability is an important determining factor for muscle glutamine uptake (transmembrane transport) and intracellular concentrations 35, 150, 151. Mittendorfer et al. estimated muscle glutamine uptake to be approximately $0.4 \mu \mathrm{mol} / 100 \mathrm{ml} \mathrm{leg} / \mathrm{min}$, which amounted to more than $50 \%$ of total muscle glutamine outward transport 35 . Part of the glutamine taken up is used for protein synthesis and part is catabolised. 


\section{ii. AV balance data}

In rats fasted for 14 hours, muscle glutamine production was $1.50 \mu \mathrm{mol} / \mathrm{kg} / \mathrm{min} 152$. In postabsorptive dogs glutamine efflux from muscle tissue was $0.8-1.5 \mu \mathrm{mol} / \mathrm{kg} / \mathrm{min} 153,154$. Starvation for 4 days, only slightly increased this production 154. In 1971 Marliss et al. reported AV-balance data of glutamine across the forearm suggesting that muscle is a major site of glutamine production in man in the postabsorptive state ${ }^{147}$. Furthermore, they observed that after prolonged starvation (5-6 weeks) a significant decrease in muscle glutamine production occurred. Feeding a meal on the other hand did not enhance glutamine efflux by skeletal muscle 35,155 . Under postabsorptive conditions, glutamine net balance across the leg in healthy volunteers was approximately $0.3 \mu \mathrm{mol} / 100 \mathrm{ml} / \mathrm{min} 35$. Assuming that leg muscle mass equals $5 \mathrm{~L}$ and that leg muscle represents $20 \%$ of total skeletal muscle mass, whole body glutamine production by skeletal muscle would be 1.7 $\mu \mathrm{mol} / \mathrm{kg} / \mathrm{h}$ on the basis of the net (i.e. total efflux minus uptake) glutamine balance data.

\section{iii. Tracer data}

Mittendorfer et al., referred in the previous paragraph, also employed glutamine isotopes and a 3-pool model to study glutamine kinetics across the leg in healthy subjects 35 . Although they found increases in intramuscular tracer enrichments, they concluded that this resulted mainly from changes in glutamine kinetics after ingestion of amino acids during the study. They estimated muscle glutamine inward flow (=total uptake) to be 0.4 $\mu \mathrm{mol} / 100 \mathrm{ml} / \mathrm{min}$ and muscle glutamine outward flow (=total output) approximately 0.7 $\mu \mathrm{mol} / 100 \mathrm{ml} / \mathrm{min}$. This was estimated to contribute approximately $50 \%$ of whole body glutamine Ra. Glutamine derived from protein breakdown was calculated to be around 0.1 . $0.2 \mu \mathrm{mol} / 100 \mathrm{ml} / \mathrm{min}$ and glutamine de novo synthesis in muscle $0.7-0.8 \mu \mathrm{mol} / 100$ $\mathrm{ml} / \mathrm{min}$. Of the total intracellular glutamine appearance rate, inward transport contributed approximately $35 \%$, glutamine derived from protein breakdown approximately $10 \%$ and de novo synthesis $55 \%$. Non-steady state conditions for intramuscular tracer enrichment, would result in underestimation of glutamine inward transport and overestimation of glutamine synthesis and glutamine outward transport.

c. Gut

i. Concentration of glutamine, GS, GA, transporters

Glutamine is quantitatively the most important fuel for the intestine. From experiments by Windmueller and Spaeth in autoperfused rat intestine, it is known that the largest part (approximately 64\%) of the glutamine carbon is oxidized to CO2. The nitrogen part appears in ammonia (38\%), citrulline (28\%), alanine $(24 \%)$ and proline $(7 \%) 5,7$. The mucosa is regarded as the metabolically most active part of the intestine. Glutamine concentrations in gut mucosa vary from $1800 \mu \mathrm{mol} / \mathrm{L}$ wet weight in rats $150,89 \mu \mathrm{mol} / \mathrm{L}$ wet weight in rats $157,29 \mu \mathrm{mol} / \mathrm{L}$ wet weight in pigs (Chapter 4 this thesis), to $43 \mu \mathrm{mol} / \mathrm{L}$ wet weight in healthy humans 158 and $28 \mu \mathrm{mol} / \mathrm{L}$ wet weight in patients without inflammatory stress (Chapter 7 this thesis). Others found higher concentrations in human duodenum $(560 \mu \mathrm{mol} / \mathrm{kg}$ wet weight) and descending colon/rectum (1140 and $1230 \mu \mathrm{mol} / \mathrm{kg}$ wet weight respectively) 159. For the sake of direct comparison some of the previous data have been recalculated to concentration per wet weight, assuming a water content of $76 \%$ in all biopsies taken. 
Glutaminase mRNA is localised within the epithelium of the villus and crypt lining of the rat intestine ${ }^{160}$. More than $80 \%$ of its total gastrointestinal activity is localised in the small intestine and largely in the mucosa ${ }^{161}$. The colon showed low GA activities in the same study. Glutaminase activities in the human gut were reported to be high in the small intestine and intermediate in the colon, whereas the proximal GI tract showed low activities 162 . In fed rats, a discrepancy has been observed between the glutaminase protein distribution and glutaminase activity is highest in the crypt protein is most abundant in the deep crypt, important enzyme regulating the crypt-villus junction 103. Quantitatively, GA is the most important enzyme regulating glutamine metabolism in small bowel mucosa 5,164 . Not only GA, but also GS is synthesized in gut mucosa. Roig et al. described in a rat model GS mRNA to be located primarily in the crypts ${ }^{43}$, but this was contradicted by Kong et al. reporting GS mRNA to be present throughout the mucosa of jejunum and ileum 105 . In rats GS activity is mainly confined to the distal stomach were almost $90 \%$ of its activity in the gastrointestinal tract is concentrated, although relative high activities were also reported in the colon 161 . GS was reported to be present both in the mucosa and in the extramucosal gastric tissues. The high GS activities in the stomach were confirmed in humans 162 .

Regulation of intestinal glutaminase activity has been more extensively studied than regulation of glutaminase mRNA and protein concentrations 55 . Among others, starvation 100,107 , ischemia reperfusion injury ${ }^{108}, 100$, sepsis 170 and IL-1 decrease glutaminase activity, in contrast to stimulating effects of small bowel resection ${ }^{171}$, glucocorticoids ${ }^{172}$, epidermal growth factor $1 \% 3$ and possibly glutamine enriched nutrition (see 55 for a more extensive review).

\section{ii. AV balance data}

$A V$-balance data regarding the intestine, usually measure the flux of substrates in the portal vein, except in small rodents where preferentially the superior mesenteric vein is used. Thus, not only fluxes across the small and large intestine, but also across other organs like the stomach, pancreas and spleen are included in these measurements. Although it is reasonable to assume that the small intestine is metabolically the most active organ within this group of organs ${ }^{58}$, differing responses of these organs to e.g. surgical stress may not be detected in this way and furthermore may dilute the measured intestinal responses. For example, after surgery the spleen increases glutamine uptake while the gut diminishes its uptake 174 . Nevertheless, in this paper we will assume that the arterial portal vein balance predominantly reflects the flux of substrate across the small intestine.

In fasted rats, gut glutamine uptake averages $3.7 \pm 0.7 \mu \mathrm{mol} / \mathrm{kg} / \mathrm{min} 152$. Lopez et al. estimated glutamine uptake by the PDV in rats to be substantially higher: $10.1 \mu \mathrm{mol} / \mathrm{kg} / \mathrm{min}$ 175. Glutamine uptake by the PDV in dogs is somewhat lower under postabsorptive conditions: $0.75-2.2 \mu \mathrm{mol} / \mathrm{kg} / \mathrm{min}$, while the results of most studies vary around 2 $\mu \mathrm{mol} / \mathrm{kg} / \mathrm{min} 111,154,176.178$. Prolonged starvation appears to increase intestinal glutamine uptake in dogs with $80-100 \% 178,179$ although not all studies confirmed this 111 . In rats, prolonged starvation similarly did not result in increased but rather decreased glutamine uptake rates ${ }^{175}$. Exercise does not change gut glutamine uptake ${ }^{111}$. In the pig model described in this thesis glutamine uptake rates across the PDV were $2.2 \mu \mathrm{mol} / \mathrm{kg} / \mathrm{min}$ under basal postabsorptive conditions (Chapter 3 , this thesis). 
The fractional extraction of glutamine by the PDV in rats is approximately $24 \% 180$. In dogs this varies from $10-20 \%$. In pigs we found extraction rates of $10-15 \%$ (Chapter 3 this thesis) 181. In otherwise healthy humans undergoing cholecystectomy, glutamine extraction was $14 \% 143$. In patients undergoing elective gastrointestinal surgery, selective sampling of the gut revealed an extraction rate for glutamine of $24 \%$ by the jejunum, $9 \%$ by the ileum and $8 \%$ by the colon 182 . No data with regard to glutamine uptake rates across the gut in humans have been reported.

\section{iii. Tracer data}

Halseth et al. determined gut glutamine uptake and release rates by incorporating a $5-15 \mathrm{~N}$ glutamine tracer in combination with AV-balance studies in dogs 111 . They reported under postabsorptive conditions a gut glutamine uptake rate of $2.6 \pm 0.5 \mu \mathrm{mol} / \mathrm{kg} / \mathrm{min}$ and an output rate of $1.0 \pm 0.7 \mu \mathrm{mol} / \mathrm{kg} / \mathrm{min}$. They estimated that gut glutamine uptake comprised $20-30 \%$ of whole body glutamine Rd and gut glutamine output $5-10 \%$ of whole body glutamine Ra. These figures were not changed by prolonged starvation nor by exercise. In pigs glutamine uptake and output rates were comparable to these figures: $3.2 \pm 0.2$ and $0.9 \pm 0.1 \mu \mathrm{mol} / \mathrm{kg} / \mathrm{min}$ respectively (Chapter 3 , this thesis). The glutamine production represented $15 \%$ of whole body Ra.

Whether glutamine is essential for the gut remains an open question. From a metabolic perspective, glutamate can be substituted for glutamine. For example, glutamate appears to be as effective as glutamine to maintain mucosal mass 183 . However, glutamine synthesis in the mucosa occurs and has been proposed to have some regulatory role, possibly regarding proliferation and differentiation of the enterocytes through gene activation 184.

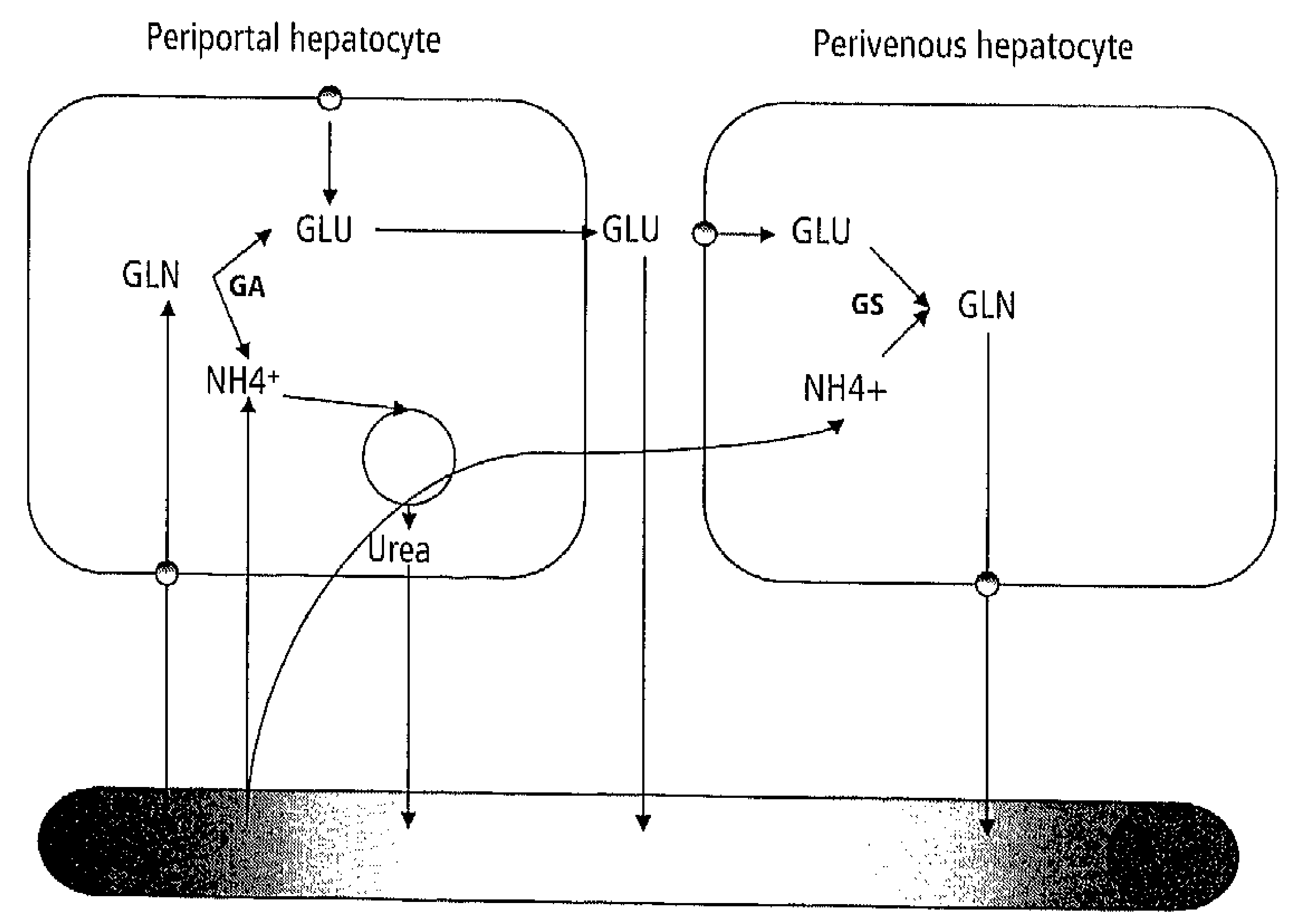

Figure 2 Schematic representation of compartmentalized hepatic glutamine metabolism 
d. Liver

i. Concentration of glutamine, GS, GA, transporters

The liver plays a central role in interorgan glutamine metabolism. Intracellular glutamine concentrations, however, are considerably lower than in muscle. In the human liver glutamine concentrations are approximately $2 \mathrm{mmol} / \mathrm{kg}$ wet weight 185 . Depending on the circumstances, the liver either takes up or releases glutamine in significant quantities. The capacity of the liver to synthesize and degrade glutamine were described first by Krebs in 1935 180. The urea cycle and glutamine turnover are key components in detoxification of ammonia and disposal of nitrogen. On the other hand glutamine synthesis in the liver is proposed to be an important mechanism for nitrogen salvage 175, 187. A clear anatomical compartmentalization of glutamine metabolism in the liver is observed 188. GS activity in the liver is relatively high and exclusively located in a small population of perivenous hepatocytes (see figure 2) 14.

The highest rates of glutamine synthesis observed in perfused rat liver were 8-10 $\mu \mathrm{mol} / \mathrm{min} / \mathrm{g}$ perivenous cells ${ }^{180}$. Compared to carbamoyl phophate synthetase, the rate limiting step in urea synthesis, the $K_{m}$ of GS in liver is substantially lower indicating that the urea cycle is a relatively low affinity/high capacity system for ammonia removal versus CS as a higher affinity/lower capacity system of. GS thus acts as a scavenger eliminating ammonia just before sinusoidal blood enters the systemic circulation. This enables the urea cycle activity to vary without the consequences of systemic hyperammonemia. This may be important particularly during metabolic acidosis. Although the source of ammonia for glutamine synthesis appear's clear, the origin of glutamate is not. Intracellular proteolysis probably only accounts for' a relatively small amount. Alternatively, synthesis of glutamate from uptake of $(x-k e t o g l u t a r a t e, ~ g l u c o s e$, lactate or from arginine-ornithine has been proposed $^{[(x)}$. (S activity in the liver appears to be relatively resistant to fluctuations by physiological and pathophysiological changes in vivo, although activities have been reported to be lower in hypophysectomized, adrenalectomized and thyroidectomized rats which could be reversed by supplementation of growth hormone, glucocorticoid or thyroid hormone respectively 100 . These hormones, however, had no effect in otherwise healthy rats. Furthermore, liver atrophy decreases total glutamine synthetase activity by decreasing GS positive cells rather than lowering the activity per cell 190. Prolonged hypoxic stress has been reported to temporally increase GS activity in liver and muscle 191.

Glutaminase expression is located periportally in contrast to GS. Glutaminase has a joint mitochondrial localization with carbamoyl phosphate, although no metabolic channel appears to exist 192. GA is only active in the presence of ammonia and is proposed to amplify the ammonia concentration to stimulate urea synthesis when an increased supply from the intestine arrives on. Brosnan's group put forward the hypothesis that the activation of glutaminase by ammonia serves to produce glutamate which is required for synthesis of $\mathrm{N}$ acetylglutamate. This in turn is an obligatory activator of carbamoyl phosphate synthase I, essential for the first step in urea synthesis ${ }^{193}$. Rates of urea synthesis are directly related to $\mathrm{N}$-acetylglutamate concentrations ${ }^{194}$. Glutaminase expression is increased during several conditions such as starvation, experimental diabetes and during high protein feeding 50. During these situations of increased supply of glutamine, glutamine is catabolized in order 
to stimulate gluconeogenesis 13 or urea production ${ }^{195}$. Furthermore, glucagon, ?-adrenergic agonists, vasopressin levels and prolonged hypoxia also increase glutaminase activity 50, 06 , 191. In fasted dogs $33 \%$ of the glutamine delivered to the portal circulation was subsequently deamidated in the liver 196.

In the liver both GS and GA are simultaneously active and results in glutamine cycling between both locations. Depending on the circumstances the net effect can be uptake of glutamine, zero balance or glutamine release. In in vitro experiments Low et al. concluded that liver glutamine utilization is controlled primarily by glutaminase activity, and to a lesser extent by system $\mathrm{N}$ transport and glutamine efflux from the cell ${ }^{197}$. The balance between glutamine release and uptake by the liver probably results from changes in glutamine breakdown, with the rate of glutamine synthesis remaining relatively constant ${ }^{100}$. .

Haüssinger et al. described effects of hepatocyte cell volume regulation on protein catabolism 198 . Glutamine probably exerts some of this effect through this mechanism since it is a potent amino acid with respect to cell swelling.

Plasma membrane transport of glutamine in the liver is through system $\mathrm{N}$ transporters. The activity of this transporter is increased in conditions which also increase urea synthesis such as administration of glucocorticoids or feeding a high protein diet 100.200 . However, some evidence points to the existence of bi-directional sodium independent transport (possibly system L) which is located in the perivenous cells 190.

\section{ii. AV balance data}

In vivo AV-balances across the liver in control-fed rats vary between slight net output or slight net utilization of glutamine 152,190 . The same holds true for dogs $(-1.6-+0.6$ $\mu \mathrm{mol} / \mathrm{kg} / \mathrm{min}) 111,154,176,178$. Prolonged starvation in dogs results in increased glutamine production, associated with enhanced ammonia uptake by the liver 111,178 and development of (compensated) metabolic acidosis ${ }^{154}$. Balance studies revealed that during exercise more glutamine was taken up by the liver than released ${ }^{154}$. In pigs we have found consistently glutamine production under postabsorptive stable conditions ${ }^{174}$ (Chapter 3 , this thesis) in the range of $0.6-0.9 \mu \mathrm{mol} / \mathrm{kg} / \mathrm{min}$. Obtaining AV-balances across the liver in healthy humans is difficult because of the ethical constraints in sampling the portal vein, which accounts for more than $70 \%$ of the blood supply to the liver. From data reported by Felig et al. in 2 different control groups no clear picture can be obtained. In the first study a glutamine uptake of $65 \mathrm{mmol} / \mathrm{L}$ across the splanchnic bed was found and in a different group but with similar characteristics an uptake of $38 \mathrm{mmol} / \mathrm{L}$ across the portal drained viscera was found, resulting in significant uptake by the liver ${ }^{144}$. However, the second study produced more equivocal results 143 . Felig summarized these findings later concluding that the human liver consumed little or no glutamine 201.

\section{iii. Tracer data}

In the isolated perfused rat liver, flux of glutamine through the glutaminase pathway was estimated to be $417 \mathrm{nmol} / \mathrm{min} / \mathrm{gram}$, which was doubled after 1 hour of glucagon treatment. The endogenous glutamine production rates by the perfused rat liver was 191 $\mathrm{nmol} / \mathrm{min} / \mathrm{g}$ under basal conditions and did not change with glucagon 192 .

Under postabsorptive conditions up to $40 \%$ of carbons utilized for hepatic gluconeogenesis 
are derived from glutamine, which can be inhibited partially by insulin 92 . With regard to the nitrogen part of glutamine, it was estimated that under basal conditions $74 \pm 18 \%$ of the glutamine taken up by the liver was channelled into urea 111 . Assuming that no steady state existed, these values overestimate the true values. However, due to the smaller hepatic intracellular glutamine pool compared to muscle, this overestimation may be minor.

Hepatic glutamine production rates in dogs have been reported to $3.6 \pm 1.2$ $\mu \mathrm{mol} / \mathrm{kg} / \mathrm{min}$ making up $\sim 35 \%$ of whole body Ra 111 . Hepatic glutamine uptake rates were $3.6 \pm 1.2 \mu \mathrm{mol} / \mathrm{kg} / \mathrm{min}$ comprising $25 \%$ of whole body Rd. In pigs, we found glutamine production rates of $3.3 \pm 1.2 \mu \mathrm{mol} / \mathrm{kg} / \mathrm{min}$, and glutamine was taken up at a rate of 2.8 $\pm 0.9 \mathrm{\mu mol} / \mathrm{kg} / \mathrm{min}$ (Chapter 3 , this thesis). Using a valine tracer protein synthesis and degradation rates we estimated glutamine de novo synthesis and oxidation rates to be 1.9 \pm 0.6 and $2.5 \pm 0.9 \mu \mathrm{mol} / \mathrm{kg} / \mathrm{min}$ respectively. This accounted for 77 and $76 \%$ of total liver glutamine production and uptake respectively. Exercise increased hepatic glutamine uptake significantly with constant glutamine output in dogs 111 . Furthermore, urea output increased significantly, possibly due to an increase in glutaminase activity. The fractional extraction rate increased under these circumstances with more than $100 \%$, a response that was attenuated in dogs starved for a prolonged time. As glutamine plasma concentrations remain stable during this time, it is likely that cell intermediary metabolism reacts to flux as opposed to concentrations. Presumably, the increased flux of glutamine through the liver during exercise is needed for gluconeogenesis, leaving nitrogen to be disposed in the form of urea. However, the situation during catabolic stress is different, because not only glutamine uptake is increased but also acute phase protein synthesis is stimulated.

\section{e. Lung \\ i. Concentration of glutamine, GS, GA, transporters}

In contrast to muscle, the glutamine pool in lung tissue is small. However glutamine synthetase is expressed to a higher degree in human lungs. Under catabolic conditions transcription and translation of GS is significantly increased allowing enhanced glutamine de novo synthesis rates $48,202.203$. Glucocorticolds are considered to be the primary mediators of GS expression in stress 204. Although GS mRNA levels increase up to 500 $700 \%$ after treatment with glucocorticoids, GS activity (and therefore the glutamine synthesis rate) only increases $20-45 \%$, probably regulated by intracellular glutamine concentrations determining GS protein stability 205.

\section{ii. AV balance data}

In rats, lungs produced significant amounts of glutamine $(8.1 \pm 2.2 \mu \mathrm{mmol} / \mathrm{kg} / \mathrm{min})$ which was 5 times more than muscle glutamine production 152 .

Data in man have been obtained in patients prior to undergoing coronary bypass surgery. In chapter 4 of this thesis, we report uptake of glutamine by the lungs $(4.4 \pm 2.8$ $\mu \mathrm{mol} / \mathrm{kg} / \mathrm{min})$. In similar patients others found the lungs to be approximately in zero balance 206, 207

To our knowledge no isotopic data on lung glutamine metabolism have been reported. 


\section{f. Other organs and cell types \\ i. Immune system}

Immune cells are metabolically very active cells but because they are not localized within one organ, their exact contribution to interorgan glutamine metabolism is difficult to quantify. However, glutamine is utilized at high rates by isolated cells of the immune system 19, 208. Metabolism is of great importance for immune cell function. Lymphocyte proliferation in response to antigenic stimuli, cytokine (IL-2, interferon-gamma, TNF- $\alpha$ ) production but also differentiation of B-lymphocytes to plasma cells are glutamine dependent processes 200, 210. Cytotoxicity of LAK cells, MHC class II expression and phagocytosis by macrophages all require the presence of glutamine 19,211.213.

Glutaminase activity is high in lymphocytes, macrophages and neutrophils 213. Glutamine nor glucose are oxidized in large quantities via the Krebs cycle but rather degraded to glutamate, aspartate and lactate. Newsholme hypothesized that the fast "stationary" cycling rate of the fuels served to allow a rapid "acceleration" of the response of immune cells to a challenge 214 .

Analogous to other immunologically active organs and tissues, the spleen contains high glutaminase activities 215 . In vivo data regarding its contribution to glutamine metabolism are scarce. In a pig model, we found that in stable postabsorptive conditions, the spleen secretes small amounts of glutamine $(0.4 \pm 0.1 \mu \mathrm{mol} / \mathrm{kg} / \mathrm{min})^{174}$.

\section{ii. Pancreas}

Glutamine is an important respiratory fuel for the pancreas $3 k$. Radioactively labelled glutamine is cleared rapidly from the circulation and the resulting activity detected in the pancreas is higher compared to other tissues 210 . Glutamine and glutamate are also involved in the regulation of insulin secretion from the pancreatic ?-cell 21. Due to its complicated venous blood drainage no in vivo measurement of glutamine kinetics across the pancreas are available.

\section{iii. Kidney}

Glutamine is crucial for two important functions of the kidney: first it is the most important donor of ammonia which is essential for proton secretion and second, the carbon skeleton is quantitatively important for gluconeogenesis $13,92,217,218$. Although in man glutamine derived from plasma is the major precursor for ammonia produced in the kidney, this does not appear to be the case in non-acidotic rats In specific circumstances glutamine catabolism by the kidney must be rapidly increased, e.g. in the presence of metabolic acidosis 219 .

Glutamine synthetase activity is low or undetectable in the human kidney as opposed to the rat, guinea pig, rabbit, sheep and mouse. It has been suggested that this may be related to the acidity of the urine 46,220 . In the rat the kidney appears to be a major source of circulating glutamine 221 . In rodents, decreases in glutamine synthesis may play a role in generating bicarbonate during metabolic acidosis ${ }^{46}$.

Glutaminase in the kidney plays a role in ammonia excretion, which in turn is of great importance in the maintenance of the acid base balance. In rat kidneys intracellular glutamate concentrations appear to regulate glutaminase activity, lower concentrations resulting in enhanced activity 222 . This may serve to regulate intracellular glutamate 
availability, as this amino acid plays a central role in intracellular amino acid metabolism. In models of metabolic acidosis, glutaminase expression increases significantly. These changes appear to be regulated by post-transcriptional mechanisms 51 .

Despite the fact that approximately $20 \%$ of plasma glutamine is filtered by the glomeruli 223 , most is reabsorbed resulting in low AV-fluxes under basal conditions in rats 219, 224. In contrast to the rat, in man the kidney extracts and metabolises significant amounts of plasma glutamine under basal conditions 97,217 . In rats under basal conditions glutamine uptake by the kidney was $1.7 \pm 0.4 \mu \mathrm{mol} / \mathrm{kg} / \mathrm{min} 152$. The kidneys of postabsorptive dogs were approximately in zero glutamine balance after a 24 hour fast, but glutamine was taken up at a rate of approximately $1.8 \mu \mathrm{mol} / \mathrm{kg} / \mathrm{min}$ after 4 days of fasting 154, 178, 225. This may be explained by an increased gluconeogenesis rate which serves as fuel for the brain 220 . It is known that during prolonged starvation the kidney an not the liver assumes the major part of gluconeogenesis $22 \%$. Renal vein and urinary ammonia output increased with $70 \%$ and $35 \%$ respectively. AV-balances in healthy humans across the kidney indicate substrate net balances of glutamine of $20-40 \mu \mathrm{mol} / \mathrm{kg} / \mathrm{min} 92,97,120$.

As expected, low AV-balances across the kidney may obscure significant glutamine uptake rates as measured with tracers. In non-acidotic rats, net glutamine flux across the kidney was quite low $(188 \pm 108 \mathrm{nmol} / \mathrm{min}$ ) but the total uptake of glutamine (measured with tracers) was $391 \pm 121 \mathrm{nmol} / \mathrm{min} 222$. This resulted in a fractional extraction of 8 $9 \%$. In healthy humans isotopic experiments employing a U-14 C glutamine tracer, estimated glutamine uptake to be $0.5-0.8 \mu \mathrm{mol} / \mathrm{kg} / \mathrm{min}$ and glutamine release by the kidney 0.1 $0.3 \mu \mathrm{mol} / \mathrm{kg} / \mathrm{min} \% 120,128,217$.

In healthy human subjects, almost one fifth of the glucose released by the kidney is derived from glutamine 120 . Approximately 70 percent of glutamine derived gluconeogenesis occurs in the kidneys $12 \%$. This is suppressed substantially by glucose ${ }^{92}$, but enhanced by epinephrine infusion 12n. On the other hand, gluconeogenesis from glutamine is not decreased in the anhepatic phase of liver transplantation 218 , illustrating the importance of the kidney (and other extrahepatic tissues) for glutamine derived gluconeogenesis. Gluconeogenesis derived from glutamine by the kidney occurred at a rate of $0.36 \cdot 0.64$ $\mu \mathrm{mol} / \mathrm{kg} / \mathrm{min}$ and this was suppressed by insulin, but stimulated by systemic epinephrine infusion 17,120 . Infusion of epinephrine increased glutamine uptake and release by the kidney, while insulin and glucagon had no significant effects on renal glutamine metabolism. The increase in fractional extraction of glutamine suggests a direct effect of epinephrine on the kidney, possibly by increasing glutamine transporter activity 126 . However, indirect effects through other mediators cannot be ruled out.

iv. Brain

GS is present in brain. Not only does glutamine play an important role as a precursor for neurotransmitters (glutamate and GABA), but also scavenging of ammonia is of great importance since ammonia is quite toxic for the brain. Using ammonia tracers, it was established that $98 \%$ of the ammonia was incorporated into glutamine. The $t 1 / 2$ of this reaction was $<3$ seconds 227 . Glutamine plays a particularly important role in cerebral nitrogen metabolism because it is virtually only through glutamine synthesis that the brain 
can dispose of ammonia. Ammonia is in excessive amounts neurotoxic and several pathophysiological conditions involving elevated ammonia levels have been described 228 . In fact excess nitrogen is released by the brain in the form of glutamine 81 . Furthermore, a role for the regulation of $\mathrm{pH}$ and water balance in the brain has also been suggested 229 . Glutamine metabolism in the brain is highly compartmentalized. GS is localized in the astrocytes whereas GA is present in neurons 228 . This permits the existence of an intercellular glutamine cycle whereby the neurotransmitter glutamate after release by nerve endings is metabolised to glutamine by astrocytes and transported back to neurons where glutamine is degraded by GA (see figure 3) ${ }^{228}$.

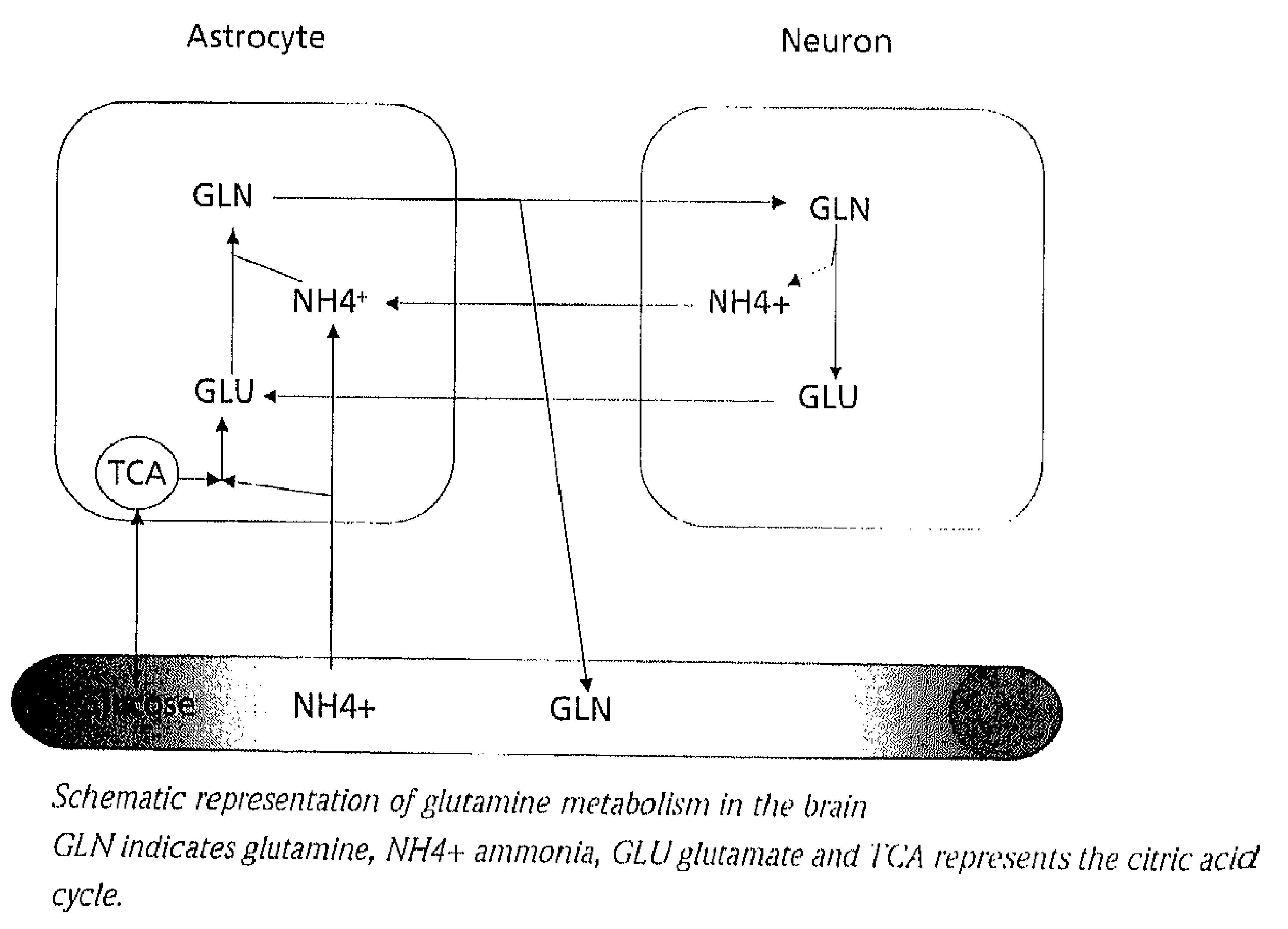

More than $80 \%$ of glutamine synthesis is accounted for by this glutamate-GABA-glutamine cycling. This cycle is a futile cycle in that it produces or releases no net glutamine. This is in contradistinction with anaplerosis (glutamine metabolized to citric acid cycle intermediates) and glutamine export which are the (opposing) determinants of (the small) net flux across the brain 230 . Moreover, a coupling with neuronal glucose oxidation has been suggested 230 . GS in glutamatergic perivascular areas is induced when hyperammonemia occurs thus protecting from neurotoxicity by synthesizing glutamine and releasing it in the circulation 231. Local glutamate concentrations possibly regulate GS distribution in the brain 231 .

\section{v. Heart}

System $N$ transport of glutamine has been established in rat and pig hearts 79 . Substantial glutaminase is present in the heart, making the assumption probable that glutamine is an important precursor for intracellular glutamate 232 . Moreover, Khogali showed that glutamine administration has protective effects in cardiac ischemia 233. Glutamate may 
have a beneficial role in cardiac hypoxia, allowing increased glycolysis without excess production of lactate. GS appears to be present in the human heart and play a role in binding of ammonia. In patients undergoing cardiac bypass surgery, glutamine (and alanine) de novo synthesis rates have been reported to be high 234 . Glutamine net balance across the heart was reported to be $2579 \pm 360 \mathrm{nmol} / \mathrm{min}$ prior to sternotomy in CABG patients.

vi. Fat

Adipose tissue is not only involved in fat and glucose but also in amino acid metabolism 235, 230. For example leucine can be transaminated and oxidized in adipose tissue 237 . Data from both animal and human experiments suggest that adipose tissue contributes significantly to glutamine interorgan metabolism 236, 238, 239. Glutamine transport in fat tissue includes both high- and low-affinity Na+dependent transporters (ASCT2 and system A respectively) and $\mathrm{Na}+$ independent transporters (system L) ${ }^{74}$. Cell swelling and adipose tissue affect glutamine transport in adipocytes, probably through effects on the ASCT2 antiporter ${ }^{74}$. Adipose tissues have been reported to contain high GS activities, probably contained within the adipocytes. The specific activity was 10-fold higher than in skeletal muscle 46 .

In healthy women a trend towards glutamine release from abdominal wall adipose tissue has been demonstrated after a 22 hour fast in both obese and lean women 239. The AV difference was $21 \pm 13(p=0.06)$. Simultaneously, net proteolysis was observed across the adipose tissue (Ra $38.0 \pm 7.0 \mathrm{nmol}$ leucine $/ \mathrm{ml} / \mathrm{min}$ ) contributing to $5.10 \%$ of whole body protcin breakdown. Previously, Frayn et al, also demonstrated net glutamine release by adipose tissue 230 .

\section{Glutamine interorgan metabolism in disease Whole body data}

In severe inflammatory diseases such as extensive burns, severe (operative) trauma, necrotizing pancreatitis or sepsis, glutamine plasma concentrations decrease substantially varying from 30 to $50 \%$ compared to healthy controls $20,116,122,211,240$, 24l. Although in uncomplicated malaria plasma glutamine concentration decrements of $30 \%$ were reported, in children with severe malaria glutamine levels were not different from controls 242 . Furthermore, survivors had significantly lower glutamine concentrations compared to those who died.

On the other hand, intake of protein deficient diets results in increased plasma glutamine concentrations in man 115 .

Apparently plasma glutamine levels do not always correlate with severity of disease and in view of the importance we attributed earlier to whole body fluxes, we will summarize results of measurements of whole body glutamine fluxes, obtained with stable isotopes in different patient categories (table 2).

\section{Surgical trauma}

We have reported that 2 days after gastrointestinal surgery whole body glutamine Ra did not change compared to preoperative control measurements despite decreased intracellular glutamine concentrations 241 (Chapter 2 , this thesis). In this study glutamine derived from de novo synthesis increased from 18 to $24 \%$. The discrepancy between a shrunken 
intracellular glutamine pool and unchanged glutamine appearance rates after surgery has also been reported in rats 243 . In contrast, we found decreased plasma glutamine appearance rates after surgery in pigs (Chapter 3 , this thesis). Whether this result can be ascribed to a methodological problem (smaller intracellular pool postoperatively resulting in a better approximation of isotopic steady state) or to true physiological changes is unclear.

\section{Short bowel syndrome and gastrointestinal disease}

Extensive bowel resection in infants results in increased glutamine plasma concentrations and a decrease in plasma glutamine Ra of approximately $30 \%$ compared to controls 244 . In a group of 5 metabolically stable adult patients suffering from a short bowel syndrome after intestinal resection, glutamine plasma concentrations were similar to controls, but again a $20 \%$ lower glutamine Ra was reported 245 . This decrease was accounted for by decreased de novo synthesis rates of glutamine. However in the early phase after enterectomy in rats muscle glutamine efflux was not affected 240 . On the basis of these data, Darmaun et al. hypothesized that glutamine turnover would be affected in patients with stable small bowel Crohn's disease. This was not confirmed however ${ }^{135}$. Carbonell et al. studied depleted but not septic patients with non-neoplastic gastrointestinal diseases such as short bowel syndrome, inflammatory bowel disease, chronic radiation enteritis and chronic pseudoobstruction 124 . In this varied population glutamine whole body rate of appearance $(2-15 \mathrm{~N}$ glutamine tracer for four hours) was increased compared to healthy controls. Data on remaining bowel length in the short bowel patients are lacking. These studies suggest that the small intestine has an important impact on glutamine metabolism. Resection of large amounts of small bowel probably result in significantly decreased glutamine consumption rates which is compensated by decreasing glutamine production. However, loss of functional intestinal mass must be substantial before an effect on whole body glutamine metabolism is detected.

\section{Diabetes}

The tight interrelation between glutamine and glucose metabolism has triggered several studies investigating glutamine whole body metabolism in diabetic subjects. Glutamine is an important carbon source for gluconeogenesis 13, 14. Intravenous supplementation of glutamine to supraphysiological levels increases gluconeogenesis derived from the glutamine carbon skeleton 140 . Conversely, approximately $13 \%$ of plasma glutamine carbon is derived from glucose in postabsorptive conditions ${ }^{138}$. However, hyperglycaemia as a result of glucose infusion or insulin deficiency in diabetes mellitus did not result in increased glutamine concentrations or whole body appearance rates ${ }^{45}$. Furthermore, plasma glutamine concentrations are not different from normal values in insulin dependent normoglycemic or hyperglycaemic diabetic subjects 247 . In contrast, in healthy humans glucose clamp studies indicated that during hyperinsulinemic euglycemia, but even more so during hyperinsulinemic hypoglycemia, glutamine plasma concentrations decrease 248 . During hyperinsulinemic euglycemia glutamine Ra increased (approximately 10\%) whereas hyperinsulinemic hypoglycemia resulted in unchanged glutamine fluxes 248 . These findings may be explained by the existence of a glucose-glutamine cycle. During hyperinsulinemia glutamine derived from proteolysis decreases but this is more than compensated by 
enhanced (muscle) uptake of glucose associated with increased glutamine de novo synthesis rates derived from glucose. During hypoglycemia, however, glucose uptake by (muscle) tissues remains unchanged. Furthermore, the proteolysis inhibiting actions of insulin is offset by the actions of counterregulatory hormones such as epinephrine resulting in unchanged glutamine $\mathrm{Ra} 248$. It is not clear why the results of the studies in insulin dependent diabetic patients are not concordant. Possibly the different doses of insulin given may have influenced outcome. Alternatively, the separate effects of insulin in this population on proteolysis on one side and e.g. glucose transport on the other, may differ from a healthy population. On the basis of all these observations it is likely that glutamine synthesis is at least indirectly affected by insulin activity.

\section{Critical illness}

In critically ill patients no differences were found in glutamine Ra compared to matched healthy controls 12. In this study stable isotopes were employed in a 4-hour protocol. However, the proportion of glutamine derived from de novosynthesis decreased significantly with approximately $15 \%$. This occurred in the context of increased protein turnover, which is characteristic of the severely catabolic state found in this patient population.

\section{Burns}

Burn patients show more pronounced changes in glutamine metabolism compared to postoperative patients. Gore and Jahoor found the whole body glutamine Ra in hypermetabolic burn patients to be $7.2 \pm 0.0 \mu \mathrm{mol} / \mathrm{kg} / \mathrm{min} 110$. This was significantly higher compared to healthy conlrols (4.5 $上 0.3 \mu \mathrm{mol} / \mathrm{kg} / \mathrm{min})$.

Table 2 Gilutamine whole body rate of appearance (WbRa) in different disease states in patients in [Imol/kg/min. (N)IDDM represents (Non) Insulin Dependent Diabetes Mellitus. GI represents grastrointestinal. Values are expressed as means \pm SEM

\begin{tabular}{llll}
\hline Patient population & WbRa glutamine & Glutamine Ra vs control & Reference \\
\hline Post GI surgery & $4.0 \pm 0.2$ & $=$ & 239 \\
Short bowel, infants & $9.5 \pm 2.1$ & $\downarrow$ & 242 \\
Short bowel, adults & $4.6 \pm 0.7$ & $\downarrow$ & 243 \\
Gl disease & $7.2 \pm 1.4$ & $\uparrow$ & 123 \\
Crohn's disease & $6.1 \pm 0.3$ & $=$ & 134 \\
IDDM & $4.9 \pm 0.4$ & $=$ & 94 \\
IDDM & $5.3 \pm 1.0$ & Not applicable & 245 \\
NIDDM & $4.8 \pm 0.2$ & $=$ & 122 \\
Wasting in AIDS & $7.1 *$ & $\uparrow$ & 247 \\
Critically ill & $4.9 \pm 0.3$ & $=$ & 121 \\
Critically ill & $5.8 \pm 1.0$ & NA & 248 \\
Burns & $7.2 \pm 0.6$ & $\uparrow$ & 115 \\
Newborn & $7.6 \pm 1.2$ & NA & 249 \\
\hline
\end{tabular}

data derived from figure manuscript 
The mechanisms underlying the changes observed have only been studied in part. On the one hand resection of organs actively involved in glutamine metabolism, such as the intestine or liver may have direct effects. On the other hand, several diseases (possibly through a common mechanism i.e. the inflammatory response) result in (neuro-Jendocrine changes which have direct effects on whole body glutamine metabolism. However complex interactions exist as demonstrated in experimental human models in which glucocorticoid infusion results in increased whole body fluxes of glutamine 120, 252 which are reversed when epinephrine and glucagon were administered simultaneously 110. Moreover, as discussed in the previous sections, cytokines and other inflammatory mediators also exert effects on glutamine synthesis, transport and breakdown rates.

In conclusion, (severe) disease generally results in decreased glutamine plasma levels but this is usually not accompanied by significant changes in glutamine turnover rates, with the exception of severely burned patients. This is in contrast with the increase in muscle glutam ine release and substantial decreases in free muscle glutamine concentrations in these conditions (see next section). Therefore, these changes are probably offset by lower glutamine production rates in other organs, resulting in similar glutamine rates of appearance compared to basal conditions. Since the currently published data on whole body glutamine kinetics in vivo reflect plasma transport rates rather than glutamine fluxes 22,84 these conclusions should be interpreted with caution. However, it remains very likely that resection of major glutamine consuming organs (and probably producing organs) directly affect glutamine metabolism $31,24 \%$.

Muscle

i. Concentration of glutamine, GS, GA, transporters

Ashkenazi et al. were among the first to observe that trauma results in a rapid and severe decrease in muscle glutamine concentration 253 . Biolo et al. reported muscle free glutamine concentrations of $4.47 \pm 1.71 \mathrm{mmol} / \mathrm{ml}$ intracellular water in burn patients, which was approximately $40 \%$ of the value found in healthy controls 18 . In critically ill patients decreases of $30 \%$ have been reported 250 . We have reported decreases of $27 \pm 0 \%$ in muscle glutamine concentrations after gastrointestinal surgery 241 . Blomqvist reported muscle glutamine decreases of $16 \%$ after total hip replacement 106 . Concentrations decreased from $10.82 \pm 0.75$ to $7.69 \pm 0.59 \mathrm{mmol} / \mathrm{L}$ intracellular water. Thus the degree of muscle glutamine decrements appear to be related to the severity of injury. Moreover, Roth et al. reported that decrements in muscle glutamine concentrations in septic patients were significantly correlated with survival 254.

On the other hand, (protein) starvation appears to increase glutamine muscle concentrations. In dogs fed protein restricted diets, glutamine concentrations tended to increase, but significantly increased when dexamethasone was administered in addition 255 .

Dexamethasone increases GS expression in muscle 256 . GS activity nearly doubles, preceded by a several fold increase in GS mRNA in skeletal muscle whereas no changes in GA activity occurred. In septic rats decreased glutamine concentrations in muscle were accompanied by an increase in maximal GS activity in some but not all muscles 257 . Furthermore, GA activity was decreased in all muscles. The depletion in muscle glutamine concentration suggests that upregulation of GS expression cannot fully compensate for the increased release rates 258 . Lukaszewicz implicated glucocorticoids as an important but not 
exclusive regulator of GS expression during infection 203. As mentioned previously, the marked losses of glutamine from the intracellular space may be explained by decreased resting membrane potentials during sepsis or endotoxinaemia 100, 101, resulting from increases in intracellular sodium and chloride concentrations and a decrease in intracellular potassium ${ }^{4}$. Since glutamine transport into the cell depends to a large extent on this membrane potential, net efflux of glutamine out of the cell into the plasma compartment occurs. This hypothesis is further supported by experiments showing that denervation of rat muscle similarly results in decreased membrane potentials, and consequently increased glutamine efflux rates ${ }^{25}$. Increased GS expression but decreased GS activities in denervated muscle have been described 259, 260.

\section{ii. AV balance data}

It has been demonstrated in several animal models that glutamine is released from muscle under catabolic conditions. After laparotomy 174, but also in models using endotoxin infusion increased glutamine efflux from the muscle has been observed 181, 258. Although we made a comparable observation on the $2^{\text {nd }}$ day after surgery, the difference reported in chapter 3 of this thesis did not reach statistical significance. In an animal model of septic shock hindquarter glutamine fluxes increased from $1.3 \pm 0.1$ to $1.9 \pm 0.3 \mu \mathrm{mol} / \mathrm{kg} / \mathrm{min}$ 181. Simultaneously, protein degradation (calculated from phenylalanine production) increased with $43 \%$. On the other hand, when compared to pairfed controls, FSR of muscle protein was not lower with the exception of the acute phase after induction, in a sepsis model with Zymosan?o1.

Clowes et al. described an increase in amino acid release from the leg during sepsis and after trauma in man 18,202. Only alanine and glutamine were released in excess of their proportion in muscle tissue 18 . Vesali et al. reported AV balances across the leg in critically ill patients measured on 3 separate occasions during 2 weeks: glutamine release varied from 90 to $115 \mathrm{nmol} / \mathrm{min} / 100 \mathrm{ml}$ leg volume 263 . These patient were being fed, but the quantity of glutamine administered had no effect on the AV balance or flux. In hypermetabolic burn patients net glutamine release by the leg was significantly elevated by a factor 3-4 compared to healthy controls 116 . However, in a similar group of patients, Biolo et al. reported glutamine release rates $(-193 \pm 52 \mathrm{nmol} / \mathrm{min} / 100 \mathrm{ml}$ leg volume $)$ which tended to be lower compared to controls, although muscle phenylalanine release rates, indicating net protein breakdown, was higher in burn patients ${ }^{98}$. Glutamine de novo synthesis rates were decreased by approximately $48 \%$ in the burn patients compared to controls. They suggested that increased transamination of pyruvate to form alanine might have limited the availability of glutamate as a precursor for glutamine.

\section{iii. Tracer data}

As discussed previously, determination of glutamine metabolism with isotopes in muscle is methodologically more problematic than in any other tissue. Although some papers have been published on this subject in burn patients 98,250 we will not discuss these data because of their uncertain physiological meaning 133. 
Gut

i. Concentration of glutamine, GS, GA, transporters

In malignant disease, glutamine concentrations in intestinal mucosa are lowered 150. In contrast, moderate surgical trauma and critical illness result in enhanced glutamine concentrations in the duodenum, whereas no change or a modest decrement was found in the (distal) colon 159,204. In chapter 4 of this thesis we describe significant decreases in jejunal glutamine concentrations after laparotomy in pigs. Therefore, differences in alterations in glutamine concentrations along the intestinal mucosa may vary not only with species but may also vary between the locations along the gastrointestinal tract and between different insults.

Kong et al. studied the effects of starvation on intestinal GA and GS mRNA levels and protein expression 205 . They reported that during starvation in the jejunum GA and GS expression decreased whereas the respective $m R N A$ levels increased. In the ileum however GS expression and GS mRNA levels decreased whereas GA mRNA increased. These results indicate that during starvation both glutamine synthesis and degradation decrease whilst the mRNA levels of the glutamine degrading enzymes increase.

Glucocorticoids increase the activity of intestinal glutaminase 62 . However, gut mucosal GA activity is decreased in endotoxinemic rats $170,200,207$.

LPS decreases glutamine transport across the brush border in rats and humans 170, 208 . Moreover, flux studies in pigs also indicate decreased glutamine uptake rates after surgery ${ }^{174}$ (Chapter 3 , this thesis) and sepsis ${ }^{181}$. But contrasting findings are reported suggesting that the effects of sepsis on transmembrane glutamine transport are only partly characterized. In vitro experiments indicated e.g. that during sepsis glutamine uptake across the basolateral membrane decreased whereas simultaneously, glutamine uptake across the brush border membrane increased 209.

Despite decreased gut glutamine uptake and GA activity during sepsis 170, 270, 271, glutamine consumption by enterocytes in vitro is increased during this condition in rats 271 , but $\mathrm{CO} 2$ production was decreased. Furthermore, the activity of glutamine transaminase was increased and DNA synthesis by the enterocytes was enhanced. This paradox can be explained by disposal of glutamine through other pathways. Glutamine synthetase, glutamine transaminase and DNA synthesis activities were for example all increased during sepsis 271 . Thus DNA and RNA but also protein synthesis may explain the increased glutamine consumption despite a decrease in oxidative decarboxylation 271, 272.

Thus, sepsis or critical illness appear to lead to decreased glutamine transport rates across the basolateral membrane of the enterocyte and diminished glutamine decarboxylation by the intestine. Simultaneously glutamine may be preferentially used for synthesis of DNA, RNA and proteins. These effects, however, may be site and species specific.

\section{ii. AV balance data}

Differing data have been reported depending on the insult and species studied.

\section{Starvation}

Miller et al. reported no change in glutamine uptake in starved dogs 225 . Others, however, described increased glutamine uptake with no change in plasma glutamine concentrations. Because in these studies liver glutamine uptake rates decreased, AV balances across the 
splanchnic bed were unchanged 58, 178, 225. Moreover, degradation shifted from transamination resulting in alanine formation to deamidation of glutamine thereby increasing the portal ammonia load.

\section{Cancer}

The fractional extraction rate of glutamine by the gut decreased from 20 to $15 \%$ in rats with advanced cancer while circulating glutamine levels fell more than a third. Furthermore, glutaminase activities in the gut decreased markedly 141 .

\section{Inflammatory mediators}

In glucocorticoid treated rats an increase in gut glutamine uptake and consumption was found $1 \%$, although this was not confirmed in a perfusion model 273 . On the other hand, endotoxin and IL-1 decrease circulating glutamine uptake by the intestine 206, 274 but administration of tumour necrosis factor alpha (TNF- $\alpha$ ) had no measurable effects in the same study.

\section{Surgical trauma}

Decreased glutamine uptake rates by the gut have been observed after surgical trauma in pigs (chapter 3, this thesis) 174. This effect was the largest on the first postoperative day (reduction of $-70 \%$ ) and slowly normalised over the ensuing days. The decrease in glutamine uptake resulted in decreased ammonia production rates. In contradistinction, increased intestinal glutamine uptake (75\% higher compared to basal values on postoperative day 2) despite falls in portal blood flow and plasma glutamine levels was described in catheterised dogs. After 4 days these changes returned to control values 170. Simultaneously, alanine efflux decreased with more than $50 \%$, suggesting other pathways for glutamine degradation.

Data in humans are very scarce. Fractional extraction rates during laparotomy in humans were $8-24 \%$. The highest rates were found in the jejunum and the lowest in the colon 170,182 . On the second day after surgery for gallstones or trauma glutamine extraction across the PDV amounted to 14-15\% 143, 275. Gut glutamine extraction in animal studies has been reported to be $7 \%$ in dogs and $20 \%$ in rats 141 . During laparotomy, net glutamine uptake by the PDV in patients was determined to be approximately $1.2 \mu \mathrm{mol} / \mathrm{kg} / \mathrm{min} 141$. During hypothermic anaesthesia in patients undergoing cardiopulmonary bypass, glutamine uptake and intestinal blood flow decreased resulting in a net gut glutamine uptake of 0.2 $\mu \mathrm{mol} / \mathrm{kg} / \mathrm{min}$, which increased to $2.4 \mu \mathrm{mol} / \mathrm{kg} / \mathrm{min}$ during rewarming, when cardiac output was restored 141 .

\section{Endotoxinemia and sepsis}

Marked changes in glutamine metabolism during sepsis and endotoxinaemia were observed since 1990. Souba et al. demonstrated a fall in gut glutamine fractional extraction from $12.0 \pm 1.6 \%$ to $2.8 \pm 0.8 \% 170$. Given the decreased portal flow during sepsis, absolute glutamine consumption by the gut is decreased even more. They observed an increase in plasma glutamine level of $13 \%$, with a decreased uptake of glutamine by the gut from 526 \pm 82 to $282 \pm 45 \mathrm{nmol} / 100 \mathrm{mg} \mathrm{BW} / \mathrm{min}{ }^{170}$. Similar findings were reported by others 266 . 
Furthermore, endotoxinemia in rats is associated with a fall in luminal glutamine uptake and mucosal GA activity 170

In fluid resuscitated septic pigs, a similar reduction in glutamine uptake by the portal drained viscera was observed $(1.3 \pm 0.1$ versus $0.4 \pm 0.1 \mu \mathrm{mol} / \mathrm{kg} / \mathrm{min}) 181$. Citrulline production did not change significantly. Phenylalanine net balance decreased from $152 \pm 32$ to $80 \pm 34 \mathrm{nmol} / \mathrm{kg} / \mathrm{min}$, indicating attenuated protein breakdown rates, or possibly increased mucosal protein synthesis rates as suggested by in vitro studies 270 .

The difference in glutamine AV balance across the PDV after surgery or with glucocorticoid treatment in comparison to endotoxinemic models can possibly be explained by suppression by endotoxin of glutamine utilization by enterocytes and immunocytes in the digestive tract 277 .

\section{iii. Tracer data}

In chapter 3 of this thesis we have described the results of surgical trauma on gut mucosal glutamine kinetics. We found that glutamine disposal in the PDV decreased significantly on POD2 $(3183 \pm 163$ versus $2161 \pm 345 \mathrm{nmol} / \mathrm{kg} / \mathrm{min}, \mathrm{p}=0.02)$, and that glutamine oxidation rates decreased proportionally. Glutamine production tended to increase to 561 $\pm 31 \mathrm{nmol} / \mathrm{kg} / \mathrm{min}$. De novo synthesis rates of glutamine, however, increased significantly after surgery. Protein breakdown rates in the PDV decreased but this decrease was not statistically significant.

Therefore in a porcine model, glutamine uptake by the PDV is decreased after surgical trauma and due to decreased GA activity glutamine disposal decreases. Glutamine de novo production, albeit in relatively small amounts, appears to increase in this situation. Currently data in critical illness are lacking.

One should bear in mind however, that the PDV not only comprise several organs but that even within intestinal tissues different tissues and cell types can exert differing and sometimes opposing effects.

Liver

i. Concentration of glutamine, GS, GA, transporters

Due to simultaneously operating high activities of both glutamine synthetase and glutaminase in the liver on the one hand and the dual blood supply by the portal vein and the hepatic artery on the othel, the liver is one of the most challenging organs to study with regard to intra- and interorgan glutamine metabolism. Furthermore, the liver harbours several cell types, such as perivenous and pericentral hepatocytes, stellate cells and Kupfer cells which are very actively but differently involved in glutamine metabolism. It is therefore not surprising that almost all data are derived from experimental studies. Furthermore, hepatic glutamine concentrations may differ significantly according to the specific site measured. Liver glutamine concentrations in advanced malignancies are reduced although in the earlier stages of the tumour, increased glutamine concentrations have been reported with a decrease in glutaminase concentration 278, 279. In septic conditions liver glutamine concentrations were not altered 261 , but the underlying mechanism is not clear.

Major changes occur in transport rates in sepsis. During endotoxinemia, a 2-3 times increase in the Vmax of glutamine transporters (system $\mathrm{N}$ ) in hepatic membrane vesicles 
has been reported, presumably due to an increase in the amount of transporters 280 . This finding was confirmed by Fischer et al. who found an increase of 2.6 times in transport rates, which was further enhanced by starvation 281 . The changes in transport are mediated by several compounds such as TNF- $\alpha$, glucocorticoids and prostaglandins 282, 283. Moreover, an additive effect of glucocorticoids and cytokines has been observed 284. However, increased intracellular metabolism due to activation of glutaminase, rather than transmembrane transport appear to cause the increased consumption of glutamine by the liver during sepsis or endotoxinemia 56,285 . During endotoxinemia, hepatic glutaminase is activated as a consequence of an increased sensitivity to inorganic phosphate 286 . Glucagon and epinephrine are proposed to be important signalling factors.

In contrast to the kidney, acidosis decreases glutamine catabolism through glutaminase and increases glutamine synthesis 287 . However, we have not been able to confirm this in pigs given $\mathrm{NH} 4 \mathrm{Cl}$ (non published observations).

\section{ii. $\quad$ AV balance data}

The increased glutamine transport rates described above, result in increased hepatic glutamine consumption in septic and endotoxin treated rats despite the absence of changes in the total activity of glutaminase 270,285 . Additionally, several other animal models indicate that in contrast to the gut, the liver changes during severe inflammatory stress from net glutamine release to significant glutamine uptake 174, 176, 288. Also in less extreme models such as surgical trauma 176 (chapter 3 of this thesis) or intensive exercise, similar findings were reported 111 . Liver resection, however, results in increased circulating glutamine concentrations 289 .

As indicated previously, another factor determining hepatic glutamine metabolism is the acid base balance: during acidosis more glutamine is produced as a consequence of a reduction in urea synthesis 290 .

In rats, endotoxinemia resulted in a 10 -fold increase in hepatic glutamine uptake resulting from enhanced liver blood flow and altered transmembrane transport of glutamine. Endotoxinemic pigs showed a significant increase in glutamine uptake by the liver compared to control $(-1191 \pm 362$ versus $-1538 \pm 312 \mathrm{nmol} / \mathrm{kg} / \mathrm{min}){ }^{181}$.

\section{iii. Tracer data}

Although data on glutamine metabolism in isolated hepatocytes are available, data on intracellular glutamine kinetics in the intact organism are scarce. In chapter 3 of this thesis describing the effects of surgical trauma in the pig, de novo glutamine synthesis is estimated to represent $33 \%$ of total Ra after surgery in contrast to $66 \%$ in the control situation. However, due to a large standard error, this was not statistically significant. Furthermore, glutamine oxidation rates after surgery, representing approximately $80 \%$ of glutamine disposal, did not differ significantly from the control values. The fate of glutamine breakdown in the liver obviously is to form glucose and urea but it can also be used for (acute phase) protein or polypeptide (e.g. glutathione) synthesis. Indeed, net protein synthesis rates are increased significantly as calculated by the net balance of valine flux ${ }^{181}$. This has also been reported in septic rats 261 . 


\section{Lung}

i.

\section{Concentration of glutamine, GS, GA, transporters}

High blood flow and availability of GS make provide the necessary conditions for the lung to synthesize glutamine in infectious states 277 . Much of the nitrogen, necessary fo $\mathrm{r}$ glutamine synthesis is taken up in the form of ammonia and glutamate. In rats pulmonary glutamine synthesis and release has been well established 202, 203, 291, 292, but also in humans who are critically ill, lung glutamine release has been demonstrated 206, 207, 293. Rat experiments show increased GS activity after laparotomy, supporting a role in glutamine interorgan metabolism ${ }^{48}$. Moreover, starvation also increased GS activity rates.

Treatment with glucocorticoids in rats doubles lung glutamine release and this is associated with increases in GS activity rather than changes in blood flow 291 . During sepsis, the GS gene is induced in the lung. In contrast to skeletal muscle, in the lung glucocorticoids only appear to partially mediate this effect 203 .

Finally, in vitro data indicate that glutamine uptake by the lungs is inhibited by glucocorticoids and endotoxin 294.

\section{ii. $\quad$ AV balance data}

Under stable control conditions in patients before elective coronary bypass surgery, the lungs take up or produce small amounts of glutamine 206, 207, 293, 205. Sepsis and burn injury in particular increase glutamine output by the lungs 206 . We estimated in chapter 5 of this thesis that in septic individuals the lungs may contribute at least $1.4 \mu \mathrm{mol} / \mathrm{kg} / \mathrm{min}$ of glutamine, i.e. $20-30 \%$ of the whole body Ra of glutamine. Part of this increase was the result of increased cardiac output. Others however, have reported efflux rates which are approximately a factor 4 higher 200, 207. This possibly results from the inclusion of burn patients in the study population as discussed in chapter 5 of this thesis. Effects of surgical trauma on lung glutamine production are less clear 207, 295. Cardiac surgery performed under hypothermic conditions modulates the metabolic response precluding conclusions with regard to lung glutamine metabolism in other types of (surgical) trauma 296.

\section{iii. Tracer data}

To our knowledge no tracer studies have been conducted reporting pulmonary glutamine kinetics. On the basis of phenylalanine fluxes we estimated that at least $44 \%$ of the glutamine released by the lungs was due to de novo synthesis 293 .

\section{Other organ (systems)}

i. Immune system/spleen

Although no direct data are available, during systemic inflammatory states, lymphoid cells most likely increase glutamine consumption considerably 210 . Immunocytes are known to proliferate rapidly after trauma or during infection. Furthermore a 10-fold increase in glutamine consumption has been demonstrated in vitro in proliferating cells compared to quiescent cells ${ }^{19}$. Furthermore, in vitro experiments indicate clearly that a wide variety of immune cells depend on glutamine for proliferation, differentiation and diverse functions such as cytokine production, phagocytosis and bacterial killing 210 .

The only organ that is considered to consist largely of lymphoid tissue and which is 
accessible for arteriovenous balance measurements is the spleen. In a catheterized porcine model Deutz et al. measured splenic fluxes after laparotomy and reported glutamine uptake $(-0.7 \pm 0.2 \mu \mathrm{mol} / \mathrm{kg} / \mathrm{min})$ contrary to a small glutamine production under control situations. This occurred despite a decreased plasma glutamine concentration 174.

\section{ii. Kidney}

Strong adaptive increases in amounts and activity of glutaminase occur in the context of acidosis 219,287 . Especially in acidotic conditions, but also after surgical trauma glutamine consumption by the kidney is increased 57, 176, 287, 292, although we found no significant changes postoperatively as described in chapter 3 . This effect is mediated by blood pH, glucocorticoids 297 and epinephrine ${ }^{92}$. This glutamine uptake may serve ammonia generation which is reabsorbed, thus readjusting the acid base balance 290 . Furthermore, the carbon skeleton of glutamine is an important substrate for renal glucose production.

On the other hand, Austgen et al. indicated that glutamine uptake changed to glutamine release by the kidney in rats being treated with endotoxin 298 . This was associated with a $50 \%$ increase in GS activity. The kidneys of these animals showed changes indicative of damage.

\section{iii. Brain}

Several disease states are associated with increased ammonia levels, which in turn result in enhanced glutamine concentrations. In encephalopathic patients increased arterial ammonia concentrations correlated to cerebral glutamine concentrations ${ }^{299}$. However, this excess of glutamine appears to have neurotoxic effects, possibly due to osmotic effects on astrocytes, resulting in impaired astrocyte function 228 . Another hypothesis suggests that elevated levels of glutamine may impair mitochondrial function resulting in increased oxidative stress and astrocytes swelling ${ }^{300}$. Analogous to the liver, acidosis results in enhanced glutamine synthesis in astrocytes 287 . During acute hyperammonemia conversion of the TCA cycle intermediate oxaloacetate to $\alpha$-ketoglutarate is followed by glutamate synthesis which is the substrate for GS. This route represents a smaller proportion (a third) of total glutamine synthesis compared to glutamine-glutamate cycling 230 .

No data are available that elucidate the role of the brain in interorgan glutamine metabolism in the injured or ill organism.

\section{$V$ Effect of glutamine supplementation on glutamine interorgan metabolism}

Glutamine provided by the enteral route is absorbed readily 301 and taken up in significant amounts by the splanchnic bed 42,118 . Approximately half of a large dose of enterally administered glutamine (as a free amino acid) was extracted by the splanchnic bed, i.e. absorbed from the intestinal lumen and passed into the portal circulation ${ }^{42}$. When only tracer doses of glutamine are given, this varies from $54-74 \%$, suggesting that the amount of glutamine administered might affect its absorption 42,118 . In newborns with enteral infusion of free glutamine with a mixed meal no change in whole body Ra was found 127. However, this can be explained by different scenarios: either absorption of differing amounts of glutamine which subsequently are degraded in the intestinal wall or the liver, compensation of the increased flow from glutamine to the systemic circulation by lower glutamine flux out of e.g. the muscle, or even no absorption of glutamine by the gut at all 
(although highly unlikely). Therefore only studies focusing on interorgan metabolism can give indications about absorption of enterally administered glutamine.

The effects of glutamine supplementation will therefore probably differ depending on the route which is used. Furthermore, since the amount of glutamine administered 42 , and the form in which it is given (in free form or with a mixed meal) may determine the degree of splanchnic extraction (up to $76 \%$ ) ${ }^{35}$, this review will largely focus on quantitative data obtained during parenteral administration of glutamine. However, qualitative data obtained from studies regarding enterally administered glutamine supplemented nutrition will also be discussed.

After summarizing the effects of glutamine administration on whole body glutamine and protein metabolism, changes induced in the respective organs focusing on glutamine concentrations, glutaminase and glutamine synthetase, protein metabolism and finally glutamine fluxes will be reviewed in detail.

\section{Handling of dipeptides}

Since glutamine used for intravenous infusion is often administered in the form of dipeptides for the sake of its stability in parenteral nutrition solutions ${ }^{302}$, information regarding the efficiency of breakdown of these dipeptides is very relevant. Liver, gut, kidney and muscle participate in clearance of the dipeptides glycyl-glutamine and alanyl-glutamine from the plasma in dogs 303 . Muscle consumed only a small part, whereas approximately 10 and $20 \%$ was taken up by the gut and liver respectively. Approximately $40 \%$ of glycyl-glutamine was cleared by the kidney. Even higher percentages of renal clearance have been suggested for this dipeptide 290,304, 305. Alanyl-glutamine, however, is cleared at a slower rate, and less by the kidney but more by muscle.

In healthy volunteers the kidney similarly played a major role in clearance of the dipeptides, but muscle tissue was more important whereas the splanchnic contribution was lower ${ }^{306}$. The dipeptides are hydrolyzed quickly (within minutes) yielding the respective constituents. The site of hydrolysis has been suggested to be extracellular rather than intracellular 307 .

\section{Whole body effects}

\section{Glutamine plasma concentrations}

Elevation of plasma glutamine levels has been demonstrated repeatedly in rat, dog and other models receiving glutamine enriched total parenteral solution both in health 178,308 , 309 and under septic or catabolic conditions 255, 310-312. Animal models using enterally administered glutamine enriched nutrition similarly increased plasma glutamine levels 54 , 313. In other studies involving both animals but also humans $127,314.319$ and as described in chapters 6 and 8 of this thesis this could not be demonstrated (table 3). Although in a posthoc analysis it was suggested that severity of illness negatively influences effects on plasma glutamine concentrations 320 , this was not confirmed in many other studies summarized in table 3. More consistent results were seen in the effects on muscle glutamine levels which were higher in the supplemented groups compared to control groups.

Glutamine enriched enteral nutrition also provided equivocal results with regard to effects on plasma glutamine concentrations in humans. Whereas short term enteral infusion 
Table 3 Effects of parenteral glutamine supplementation on glutamine (GIn) plasma and muscle concentrations. An arrow pointing upwards indicates increase, an arrow pointing downwards decrease and = indicates no change compared to control conditions.

\begin{tabular}{|c|c|c|c|c|}
\hline $\begin{array}{l}\text { Reference } \\
\text { no. }\end{array}$ & $\begin{array}{l}\text { Gln dose } \\
(\mathrm{g} / \mathrm{kg} / \text { day })\end{array}$ & Population & GIn plasma & Gln muscle \\
\hline$\overline{317}$ & $0.29-0.57$ & Healthy subjects & $\uparrow$ & \\
\hline 318 & $0.29-0.57$ & Healthy subjects & $\uparrow$ & \\
\hline 319 & 0.19 & Postoperative Gl & $=$ & $\uparrow @$ \\
\hline 106 & 0.24 & Cholecystectomy & & $\uparrow @$ \\
\hline 320 & 0.29 & Postop abdominal surgery & & $\downarrow *$ \\
\hline 321 & 0.28 & Postoperative Gl & $=\wedge$ & $\downarrow(48 \%)$ \\
\hline 105 & 0.28 & Hip surgery & $\uparrow @$ & $\uparrow @$ \\
\hline 322 & 0.3 & Postoperative GI & $={ }^{*}$ & \\
\hline 323 & 0.34 & Postoperative Gl & $\uparrow @$ & \\
\hline 316 & 0.28 & Healthy subjects & $=$ & \\
\hline 107 & 0.36 & Cholecystectomy & & $\uparrow @$ \\
\hline 324 & 0.34 & Postoperative Gl & $\uparrow$ & \\
\hline 325 & 0.21 & Preop colorectal cancer/IBD & $=$ & \\
\hline 326 & 0.36 & Chemotx for haematological malignancy & $=$ & \\
\hline 327 & $18 \mathrm{~g} /$ day & ICU & $=$ & \\
\hline 102 & $25 \mathrm{~g} /$ day & ICU & $=$ & $=$ \\
\hline 41 & $\#$ & Newborn & $\uparrow$ & \\
\hline 328 & $0.5(20 \%$ of $A A)$ & Newborn (extremely low birth weight) & $\uparrow$ & \\
\hline 329 & 0.3 & Postoperative & $=\dagger$ & \\
\hline 330 & 0.57 & BMT & $\uparrow$ & \\
\hline 331 & 0.4 & ICU & $\uparrow$ & \\
\hline 332 & 0.23 & Preop TPN GI cancer/IBD & $\uparrow$ & \\
\hline 333 & 0.4 & $\mathrm{ICU}$ & $\uparrow$ & \\
\hline 314 & $\#$ & ICU & $=$ & \\
\hline 313 & 0.35 & Trauma & $=$ & \\
\hline 334 & $20 \mathrm{~g} /$ day & TPN & $=$ & \\
\hline 335 & 0.56 & Postop rectal cancer (day 1) & $\uparrow(\uparrow 4 \%)$ & $=(-13 \%)$ \\
\hline 335 & 0.56 & Postop rectal cancer (day 3) & $\uparrow(7 \%)$ & $\downarrow(-41 \%)$ \\
\hline 336 & 0.3 & $\mathrm{ICU}$ & $=\uparrow \$$ & \\
\hline 337 & 0.5 & Burn patients & $\uparrow$ & \\
\hline 338 & 0.4 & $\mathrm{ICU}$ & $\uparrow$ & \\
\hline 104 & $0.28-0.86$ & ICU & $\uparrow$ & $=$ \\
\hline
\end{tabular}

* higher compared to control group $\$$ only increased if TPN was given $>9$ days

@ no change compared to basal values in glutamine \# data not given

supplemented group; i.e no change compared to $\wedge$ no change compared to preop level(other group $\mathrm{Gln}+\mathrm{GH}$ ) decrease in control group

$\dagger$ similar recovery compared to glutamine 
of glutamine in healthy subjects did increase plasma glutamine concentrations $35,104,301$, 344 , others found no change 316 in a similar population after 5 days of glutamine enriched enteral nutrition. Furthermore, in very low birth weight infants fed enterally with a glutamine supplemented or a control enteral formula either with or without additional parenteral nutrition, plasma glutamine concentrations were not increased 345,346 . Moreover, also under catabolic conditions such as critical illness and trauma or in glucocorticoid treated subjects, glutamine enriched enteral nutrition was reported not to result in enhanced plasma glutamine concentrations in some studies $\lceil 30,317-319,347,348$ in contrast to other publications $349 \cdot 352$.

It is important to realize that repletion of plasma glutamine concentrations not necessarily equals restoration of tissue glutamine levels. For example, in rats depleted in glutamine by treatment with methionine sulfoximine, intravenous glutamine infusion restored plasma glutamine concentrations but replenished lung glutamine stores to only $65 \%$ of the control values 205 . Other examples in humans in whom plasma glutamine levels stabilized or increased but with simultaneous decrements in muscle glutamine concentrations can be found in table 3. An important factor determining the equilibrium between the plasma and tissue compartment is time and amount of glutamine infusion because of effects on (up)regulation of transporters, effects on GA, GS and protein turnover.

\section{Protein metabolism}

Glutamine has been thought to affect protein turnover, and particularly protein synthesis 26. On the other hand, protein synthesis and breakdown rates directly affect glutamine metabolism. Glutamine enriched TPN in tumor bearing rats decreased whole body protein breakdown rates 353 , whereas in a septic model whole body protein synthesis and breakdown rates have been reported by the same author either to increase or to remain unchanged 310,354. In contrast in septic or irradiated rats alanyl-glutamine infusion decreased whole-body proteolysis more than protein synthesis, resulting in an improved protein net balance 355,356 . In hypercatabolic dogs enteral glutamine supplementation decreased leucine oxidation and improved net leucine balance 313 . No effects of glutamine were reported on leucine kinetics after 7 hours of parenteral glutamine administration in a catabolic dog model 255. However, in several patient populations, positive effects of glutamine enriched parenteral nutrition on nitrogen balance have been reported $323,324,326$, 334, 357. The reliability of nitrogen balance in the context of changing glutamine concentrations, on the other hand, has been questioned 358 .

In non stressed healthy subjects, enteral glutamine supplementation had minor effects on whole body protein turnover 344,359 . Furthermore, leucine oxidation rates and phenylalanine hydroxylation rates were not affected by glutamine enrichment of the enteral diet 359 . Leucine kinetics did not change in undernourished or growth delayed but stable children with cystic fibrosis receiving oral supplements of glutamine for 4 weeks 351 . In corticosteroid-treated volunteers, enteral glutamine supplementation for 2 days did not change leucine and phenylalanine kinetics ${ }^{130}$. In critically ill trauma patients no effects were noted of enteral glutamine supplementation on whole body protein turnover, synthesis and breakdown rates ${ }^{347}$. Similarly in premature infants, glutamine supplementation via the 
enteral route did not change leucine kinetics ${ }^{346}$. Also in burned children, enteral glutamine supplementation did not increase net protein accretion 348 .

Thus although in animals glutamine supplementation may have an anabolic effect on whole body protein metabolism, robust data are lacking in man, both under stable and in catabolic conditions.

\section{Glutamine flux}

Parenteral glutamine administration composes an additional factor in whole body glutamine flux. In chapter 9 of this thesis we describe that in metabolically stable pigs glutamine enriched TPN indeed increases whole body glutamine Ra. In catabolic (postoperative) piglets, however, glutamine rate of appearance was not changed by exogenous glutamine infusion $(36 \mu \mathrm{g} / \mathrm{kg} / \mathrm{min}){ }^{360}$.

In nutritionally depleted, but metabolically stable patients needing TPN we found that the whole body Ra of glutamine increased significantly with the amount of glutamine which was infused ${ }^{329}$ (chapter 7 of this thesis). Nor glutamine derived from protein breakdown, nor glutamine de novo synthesis rates changed significantly.

Jackson et al. studied the effects of glutamine supplemented total parenteral nutrition on whole body glutamine Ra in critically ill patients 337 . They similarly reported an increase in glutamine flux of $5.2 \pm 0.5$ to $7.4 \pm 0.7 \mu \mathrm{mol} / \mathrm{kg} / \mathrm{min}$ which was fully accounted for by the additional glutamine which was infused. A small increase was found in the relative contribution of glutamine de novo synthesis to the endogenous glutamine Ra ( $68 \pm 3$ versus $60 \pm 3 \%$ ). It should be noted however, that these results compared fasted conditions at baseline with fed conditions during administration of TPN.

In contrast to the results of parenteral glutamine administration, effects of enteral glutamine supplementation on whole body glutamine Ra have been more confusing. In enterally fed pigs (including glutamine at a rate of $219 \mu \mathrm{mol} / \mathrm{kg} / \mathrm{h}$ !) glutamine flux was very high amounting to $15.6 \mu \mathrm{mol} / \mathrm{kg} / \mathrm{min} 361$. Hankard et al. infused a large load of free glutamine enterally over 5 hours in healthy volunteers 42 . They reported an increased (total) glutamine Ra, but a simultaneous decrease of $20-30 \%$ in endogenous glutamine Ra (equal to total Ra - minus proportion of glutamine administered reaching the systemic circulation), a decline in glutamine de novo synthesis and no change in glutamine derived from protein breakdown. Others, however, found no effect of glutamine enriched enteral nutrition on endogenous glutamine Ra in healthy subjects ${ }^{35}$. Furthermore, the contributions of de novo synthesis and protein breakdown to glutamine production remained unchanged. Oral glutamine supplementation to children with Duchenne's dystrophy resulted in increased total glutamine Ra although the endogenous Ra decreased 362 . Both glutamine de novo synthesis and glutamine derived from protein breakdown decreased.

In healthy subjects treated with corticosteroids, enterally administered glutamine did not change glutamine rate of appearance, nor the proportion of glutamine derived from de novo synthesis in the fasted state ${ }^{130}$. But a decrease in glutamine Ra and glutamine de novo synthesis was reported in the fed state. In contrast, Humbert et al. found that in a similar model in dogs (also treated with corticosteroids), enteral glutamine supplementation (1.15 $\mu \mathrm{mol} / \mathrm{kg} / \mathrm{h}$ ) increased glutamine rate of appearance in the fed state with $56 \%$ from 17.8 
to $27.8 \mu \mathrm{mol} / \mathrm{kg} / \mathrm{min}$, which is substantially higher compared to values obtained in fasted dogs (approximately $10 \mu \mathrm{mol} / \mathrm{kg} / \mathrm{h} 111$, and in humans Itable 1 ) 313 . Whole body Ra of glutamine and the endogenous glutamine flux in premature infants fed parenterally and enterally did not increase by enteral glutamine supplementation $10.3 \mathrm{~g} / \mathrm{kg} /$ day $\}^{3.4 t}$. In this group de novo synthesis accounted for $80 \%$ of the glutamine $\mathrm{Ra}$, comparable to findings in adults and older children 244 . In children with cystic fibrosis, glutamine fluxes were not changed by oral glutamine supplementation 351 .

In conclusion, glutamine enriched parenteral nutrition appears not to affect endogenous glutamine flux thus resulting in increased total appearance rates. The effects of enteral glutamine supplementation, however, are more diverse. Short infusion studies with large amounts of glutamine seem to increase whole body glutamine Ra, but in general with longer periods of glutamine supplementation no evident increase in glutamine Ra was observed. Possibly, enhanced uptake of the absorbed glutamine by liver after up regulation of transporters and glutamine degrading enzymes may help explaining this difference between enteral and parenteral nutrition.

\section{Muscle}

\section{Glutamine concentrations}

Glutamine supplemented enteral diets in rats did not increase plasma or muscle glutamine concentrations 50.308 . However, in rats glutamine enriched TPN maintained muscle glutamine concentrations in contrast to glutamine free TPN fed animals ${ }^{308}$. In dogs under catabolic conditions short term intravenous infusion of glutamine $(800 \mu \mathrm{mol} / \mathrm{kg} / \mathrm{h})$ did not increase muscle glutamine concentrations although an increase in plasma glutamine was reported ${ }^{255}$. Results of glutamine supplemented parenteral nutrition on muscle glutamine concentrations are summarized in table 3 . From these data it can be concluded that glutamine supplementation maximally results in maintenance of intracellular glutamine concentrations possibly depending on the intensity of the catabolic insult.

Short term enteral glutamine administration in healthy subjects did not change muscle glutamine concentrations despite increased plasma levels ${ }^{35}$. But when glucose was added to the glutamine, intramuscular glutamine concentrations decreased which was associated with a decrease in de novo glutamine synthesis. This was suggested to be the result of an inhibitory effect of insulin on GS activity. Finally, in critically ill patients, reduced muscle glutamine concentrations could not be altered by enteral glutamine supplementation 350 .

\section{GS and GA}

Enteral glutamine supplementation resulted in decreased glutamine synthetase activities in muscle in rats ${ }^{59}$. Supportive evidence for this phenomenon has also been observed in vitro, in which increased intracellular glutamine concentrations enhance GS degradation 51,53 , 363. Glutaminase activity in skeletal muscle, however, does not appear to be sensitive to glutamine concentrations 53 .

\section{Protein metabolism}

Most studies regarding the effects of glutamine on protein metabolism have been performed 
in muscle. In non stressed pigs, we found a change to net protein accretion in the hindquarter in the presence of glutamine enriched TPN (chapter 8 , this thesis). This was mainly the result of decreased protein breakdown. This decrease in muscle protein breakdown was also reported in rats 354 . In catabolic rodents, muscle fractional synthetic protein synthesis rates were enhanced by glutamine supplementation $353,354,364$, although not in all studies 356 . In healthy subjects, short term infusion of enteral glutamine increased muscle protein synthesis rates 35 . Parenteral glutamine supplementation after total hip replacement resulted in unchanged ribosome concentrations instead of a decrease as seen in the control group, suggestive of maintained protein synthesis rates 106 . In patients after gastrointestinal surgery, glutamine supplemented TPN resulted in decreased muscle protein FSR although the concentration of ribosomes remained unchanged ${ }^{325}$. The degree of this decrease was similar to that observed in patients with glutamine free TPN after surgery 365 . Moreover, in ICU patients no effect of parenteral glutamine supplementation on muscle protein synthesis or protein content was found ${ }^{105}$. In earlier studies, however, Hammarqvist et al. demonstrated an attenuating effect of glutamine enriched TPN on the fall of muscle protein FSR after elective surgery 107, 324.

\section{Glutamine flux}

In dogs, infusion of glutamine $(3 \mu \mathrm{mol} / \mathrm{kg} / \mathrm{min})$ did not change glutamine efflux from the hindlimb ${ }^{154}$. Higher infusion rates of glutamine $(10 \mu \mathrm{mol} / \mathrm{kg} / \mathrm{min})$ either in the free form or as a dipeptide, resulted after 1 hour in decreased efflux from muscle in anaesthetized dogs $(0.03 \pm 0.2$ versus an uptake of $0.47 \pm 0.23 \mu \mathrm{mol} / \mathrm{kg} / \mathrm{min}$ for alanyl-glutamine and free glutamine respectively) ${ }^{153}$. In chapter 8 of this thesis we report that glutamine enriched TPN in pigs did not change glutamine efflux from muscle tissue. Similar findings have been reported in non-stressed dogs 366 and healthy subjects 306 . The fact that net protein retention occurred in muscle, indicated that in muscle glutamine de novo synthesis was enhanced
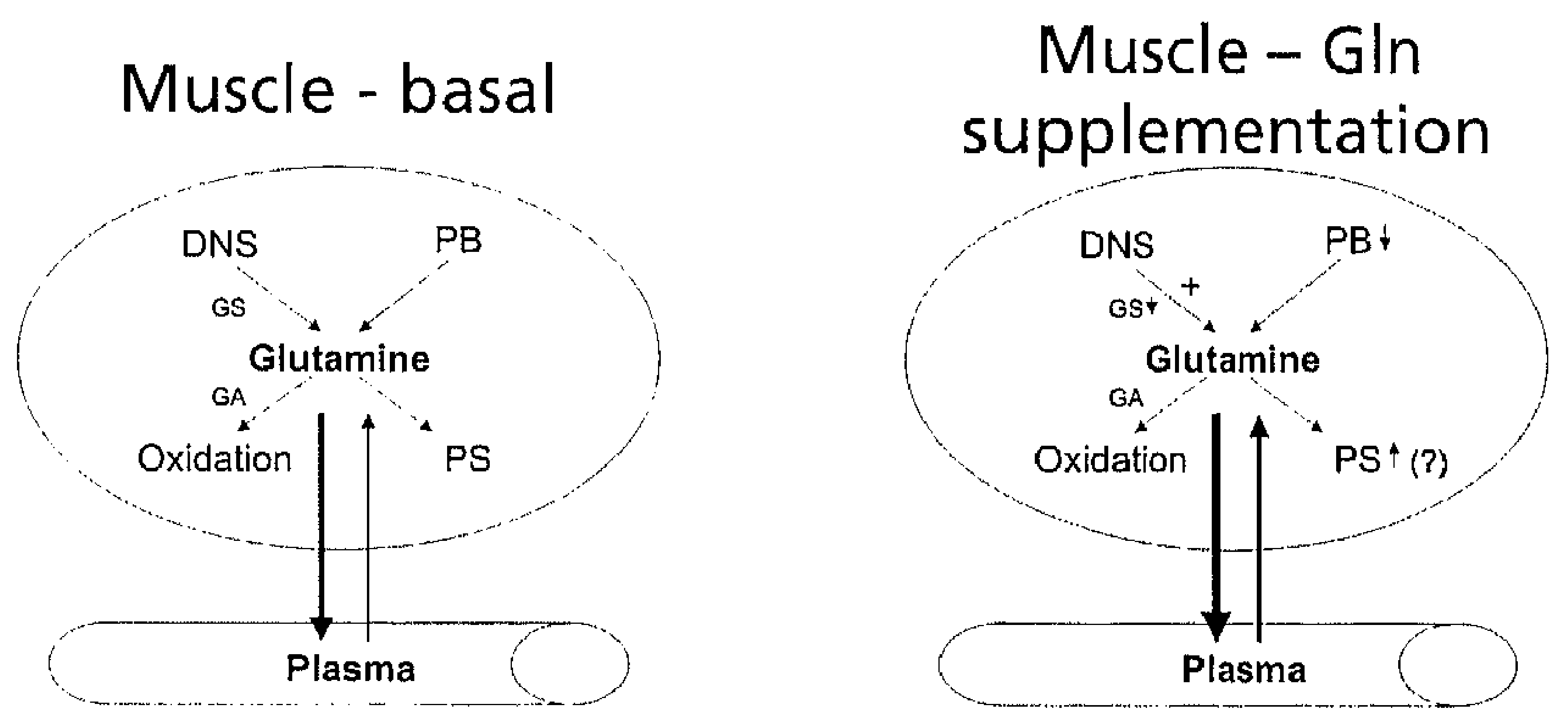

Figure 4 The oval represents the intracellular pool of the muscle. De novo synthesis (DNS) of glutamine catalyzed by glutamine synthetase (GS) and protein breakdown (PB) furnish glutamine which can disposed of by either oxidation through activated glutaminase (GA), protein synthesis (PS) or transport into the plasma compartment. + indicates increased flux, an arrow pointing upwards indicates an increase and an arrow pointing downwards reflects a decrease. 
(chapter 8 , this thesis). In healthy non-stressed humans enteral glutamine administration results in increased glutamine delivery to muscle 35 . But due to a simultaneously increased transport out of the muscle compartment, the glutamine net balance remained unchanged. In a similar group, short enteral glutamine supplementation decreased glutamine release across the leg 104 .

Glutamine release by the hindquarter in pigs shortly after surgery was not affected by low dosed parenteral glutamine supplementation 360 . However, in septic dogs fed a glutamine enriched TPN solution, glutamine efflux from muscle tissue decreased significantly 367 . In critically ill patient a 3 hour administration of enteral glutamine enriched diet did not change muscle glutamine efflux across the leg 104 .

Thus under stable conditions, parenteral glutamine supplementation appears not to affect glutamine efflux significantly although glutamine de novo synthesis is enhanced. In catabolic conditions, however, glutamine may diminish muscle glutamine release. All of these changes are summarized in figure 4.

\section{Splanchnic bed}

No human data are available to describe the effects of glutamine infusion on intestinal and liver handling of exogenously administered glutamine separately. Some data, however, have been generated describing the effects across the splanchnic bed. Lochs et al. determined that infusion of free glutamine or alanyl-glutamine increased glutamine uptake across the splanchnic bed ${ }^{306}$. Furthermore, in healthy subjects treated with glucocorticoids, extraction of glutamine by the splanchnic bed was increased ${ }^{308}$. However, in premature infants, enteral glutamine supplementation did not change the approximately $50 \%$ first pass extraction of glutamine by the splanchnic bed ${ }^{346}$.

\section{Portal drained viscera (PDV)}

The gut is one of the major consumers of glutamine. Therefore, one of the goals of administration of glutamine enriched TPN has been to provide additional glutamine for the gastrointestinal tract in order to prevent villus atrophy and increased permeability 330 .

\section{Glutamine concentrations}

In healthy growing rats, glutamine enriched parenteral nutrition did not affect gut mucosal glutamine concentrations ${ }^{308}$. Neither did enteral glutamine supplementation in hypercatabolic dogs increase mucosal glutamine content 313 . In metabolically stable pigs fed glutamine enriched TPN, we even found a decrease in glutamine concentrations in jejunal mucosa (chapter 8, this thesis). On the other hand, parenteral glutamine supplementation in addition to oral diet in patients receiving chemotherapy resulted in increased duodenal mucosa glutamine concentrations 369 . Enteral glutamine supplementation in critically ill patients only increased mucosal glutamine concentrations when given as glutamine rich proteins rather than as free glutamine 319 .

\section{$\mathrm{GA}$ and $\mathrm{GS}$}

In non-stressed rats receiving glutamine enriched TPN, intestinal glutaminase activity was not affected compared to non fed controls in contrast to lower glutaminase activity found in rats 
who were fed a glutamine free TPN solution 180 . Enteral glutamine supplementation had the same result 59 . However, in most other catabolic models it was found that glutamine supplementation increased gut glutaminase activity. Kong et al. studied the regulation of intestinal glutaminase during infusion of glutamine-enriched parenteral nutrition. Along the small intestine glutaminase activity and GA expression were both increased, although expression and activities were higher in the jejunum compared to the ileum. The rats were in a catabolic state as indicated by loss of body weight and a negative nitrogen balance 370 . They suggested that glutamine administration leads to an accumulation of glutaminase mRNA by an unknown mechanism. Other catabolic rat models confirmed increased glutaminase activities as a result of parenteral glutamine supplementation ${ }^{371-375}$. This is in contrast to decreased glutamine consumption and glutaminase activities as seen in septic rats 170 .

\section{Protein metabolism}

Intravenous glutamine supplementation in healthy or moderately stressed rats and dogs did not affect protein fractional synthesis rates in the gastrointestinal tract 370.379 . Furthermore, enteral amino acid intake but not specifically glutamine increased protein FSR in duodenal mucosa in healthy volunteers 344 . In rats, enterally supplemented glutamine failed to increase mucosal FSR ${ }^{356}$. In piglets, increased protein synthesis rates were observed after enteral glutamine administration 380 . Furthermore in catabolic animal models, glutamine enriched TPN increased enteral protein FSR 313, 353, 376. In glucocorticoid treated healthy humans receiving enteral glutamine enriched nutrition, a tendency to increased protein synthesis rates in gut mucosa was reported ${ }^{381}$. Effects on proteolysis have been studied less intensively because of technical problems. Coëffier et al. demonstrated that glutamine supplementation via the enteral route decreases $\mathrm{mRNA}$ expression of ubiquitin within 6 hours, suggesting decreased protein breakdown in the intestinal mucosa 344 . Other pathways for protein breakdown were not affected.

Thus glutamine appears to stimulate protein synthesis rates in the gut at least during catabolic conditions, and some indirect evidence suggests that protein breakdown may be slowed down.

\section{Glutamine flux}

Glutamine has been suggested to increase blood flow across the splanchnic bed although this has not been consistently found 382 (Chapter 8 , this thesis). Such an effect may have important consequences for interorgan metabolism.

Glutamine is taken up in large amounts from the circulation. Intravenous infusion of free glutamine in non or mildly catabolic animals further enhances gut glutamine uptake 152, 177, 178, 309. Glutamine added to TPN, however, does not change gut glutamine uptake in non catabolic dog and pig models 366,383 (chapter 8, this thesis) in contrast to a rat model 180. On the other hand, in stressed organisms, glutamine supplementation to TPN does enhance gut glutamine consumption $360,373,374$.

In chapter 8 of this thesis we report the effects of glutamine enriched TPN on glutamine kinetics in the PDV. Glutamine disposal decreased whereas glutamine de novo synthesis 
rates increased significantly. Simultaneously protein breakdown rates decreased but this decrease did not reach significance.

In figure 5 an overall view of changes due to glutamine infusion are represented.
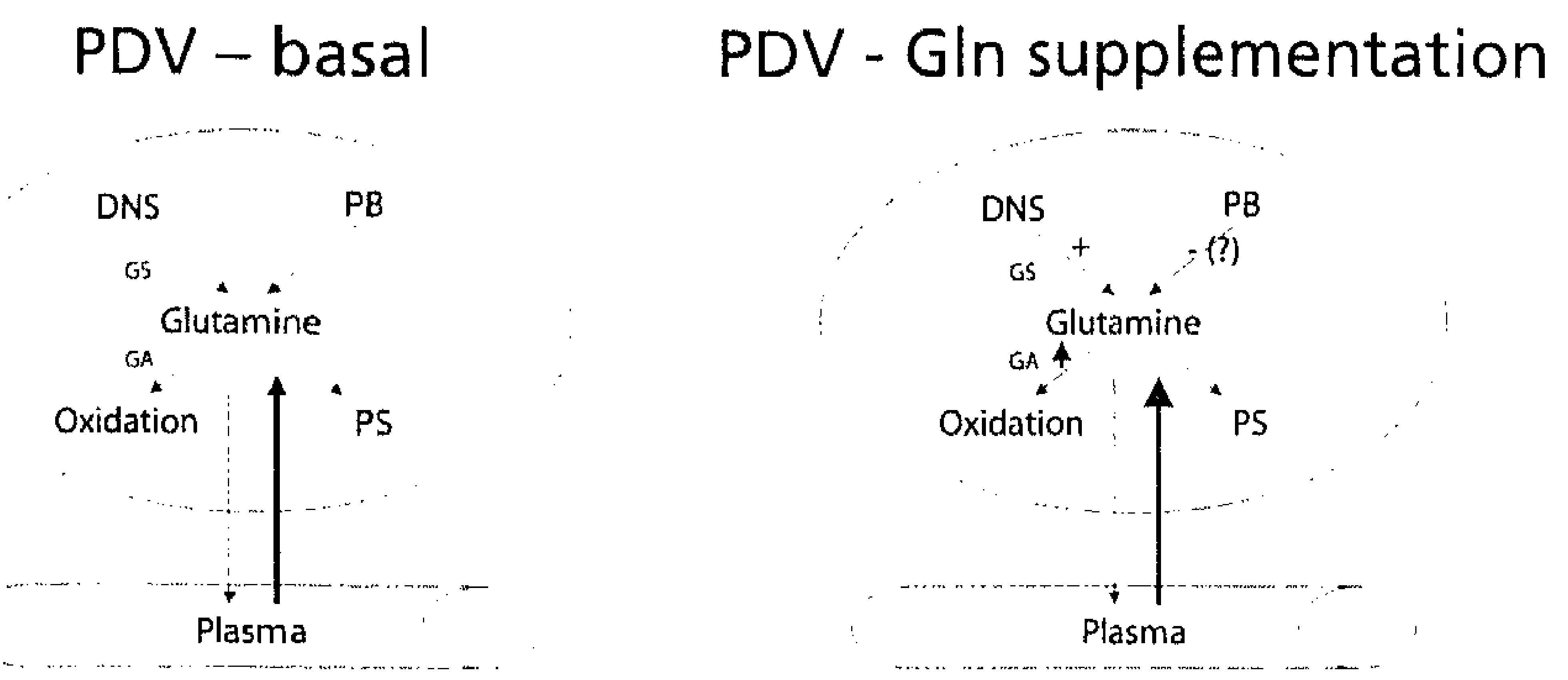

Figure 5 Effects of exogenous glutamine supplementation on intestinal glutamine metabolism. The oval represents the intracellular pool of the intestine as represented by the portal drained viscera. De novo synthesis (DNS) of glutamine catalyzed by glutamine synthetase (GS) and protein breakdown $\mid$ PB/ furnish glutamine which can disposed of by either oxidation through activated glutaminase (GA), protein synthesis (PS/ or transport into the plasma compartment. + indicates increased flux, a decreased flux, an arrow pointing upwards indicates an increase and an arrow pointing downwards reflects a decrease.

Liver

Glutamine concentrations

Liver glutamine concentrations were maintained by glutamine enriched parenteral nutrition in contrast to glutamine free TPN in rats 308.

\section{GA and GS}

The liver contains high amounts and activities of both glutamine synthetase and glutaminase. Enteral glutamine supplementation resulted in not significantly increased glutaminase activities but in significantly decreased glutamine synthetase activities 59 .

\section{Protein metabolism}

Protein synthesis rates in the liver were not changed after glutamine enriched nutrition in rat models 353,356 , but increased in septic rats receiving glutamine 354 . In pigs we found no significant change in hepatic retention of essential amino acids (chapter 8, this thesis).

\section{Glutamine flux}

Glutamine uptake by the liver 5 hours postoperatively was not changed by small amounts of glutamine supplementation 360 . However, during infusion of large amounts of free glutamine, the liver became the principal site for glutamine uptake in rats ${ }^{152}$. Moreover also in dogs glutamine extraction by the liver significantly increased when glutamine was 
administered 178, 300. Similar observations in pigs are described in chapter 8 of this thesis. Depending on the degree of metabolic stress, this additional glutamine can be used for protein or polypeptide synthesis (e.g. GSH), or oxidized e.g. to form TCA intermediates and urea.

In figure 6 these changes are displayed schematically.
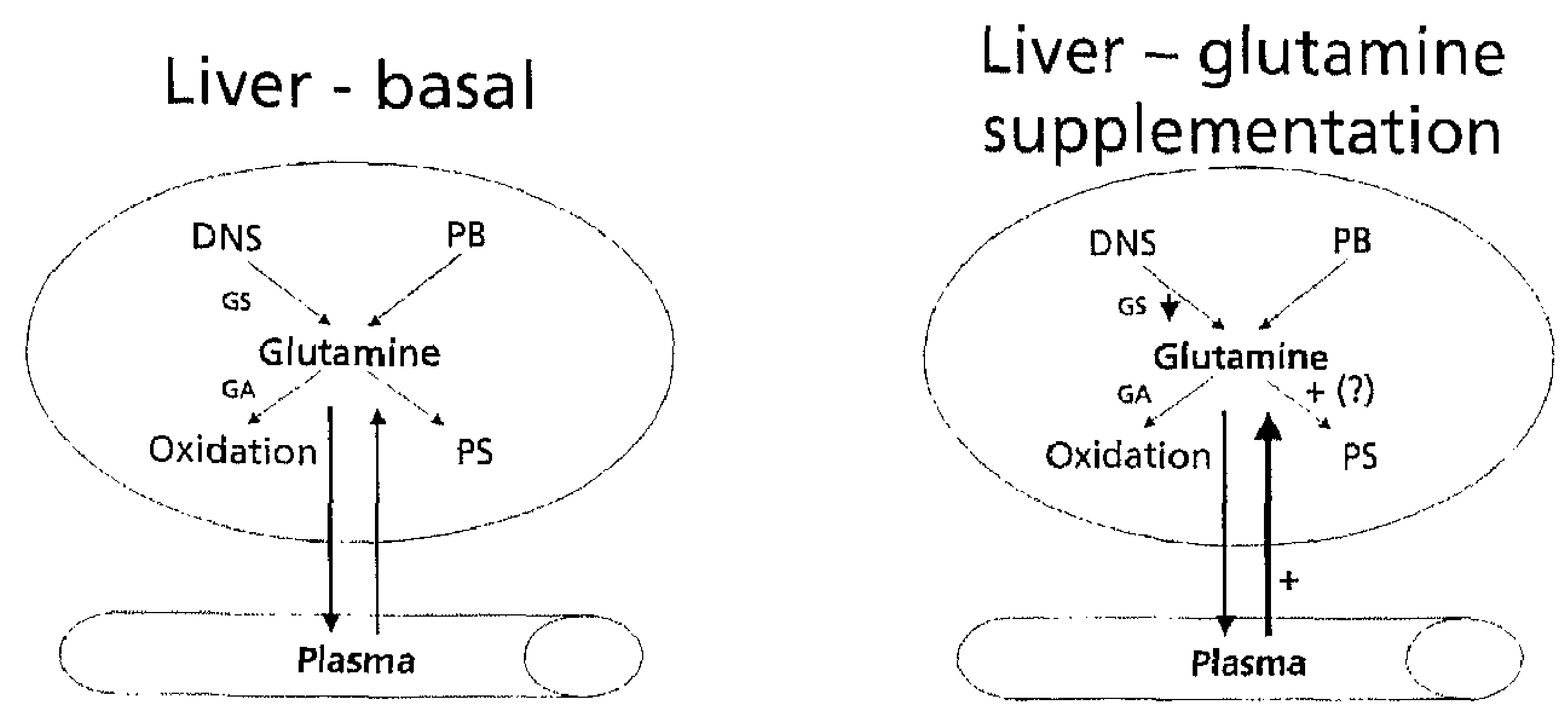

Figure $6 \quad$ Effects of exogenous glutamine supplementation on hepatic glutamine metabolism. The oval represents the intracellular pool of the intestine as represented by the portal drained viscera. De novo synthesis (DNS) of glutamine catalyzed by glutamine synthetase (GS) and protein breakdown (PB) furnish glutamine which can disposed of by either oxidation through activated glutaminase (GA), protein synthesis (PS) or transport into the plasma compartment. + indicates increased flux, an arrow pointing downwards reflects a decrease.

\section{Kidney}

As mentioned previously, the kidneys play a major role in disassembling glutamine dipeptides 154, 290. In several animal models free glutamine supplementation resulted in either increased renal glutamine consumption or no change in glutamine uptake by the kidneys $152,178,360$, although some used only very limited infusion times ${ }^{178}$. In healthy volunteers, glutamine infusion in the free form had no significant effect on kidney glutamine consumption but glutamine uptake ceased when infused as a alanyl-glutamine dipeptide 307 . When infused as glycyl-glutamine the kidneys started releasing glutamine ${ }^{305}$.

This suggests that the form of glutamine supplementation may have an important bearing on renal glutamine metabolism. Furthermore, renal glutamine metabolism during catabolic stress remains to be clarified.

\section{Spleen/immune system}

Glutamine "depletion" in severely ill patients has been suggested to cause deficient function of the immune system 211,240 . Its importance is for example suggested by rat experiments showing that dietary glutamine supplementation protected against mortality from intravenous injection of $S$. Aureus in a dose dependent manner 384 . In this study the rats with the highest glutamine intakes had the highest splenic weights.

Glutamine concentrations in the spleen of healthy growing rats, however, were not 
affected by provision of glutamine supplemented TPN 308. Splenic and thymic protein synthetic rates tended to increase in septic rats fed glutamine enriched TPN 354.

However, in vitro experiments indicated that lymphocyte glutaminase activity decreased progressively after endotoxin treatment resulting in decreased glutamine consumption 385.

\section{CONCLUSIONS}

Glutamine is a key substrate in interorgan amino acid metabolism showing significant changes in the response to injury or disease. The regulation of this response in glutamine metabolism appears to occur at multiple levels (e.g. glutamine transport, glutamine synthesis and catabolism) and through a variety of mechanisms (glucocorticoids, epinephrine, insulin, glucagon, neural stimuli, cellular hydration mechanisms, GH, IGF-1 etc.). This overview aims to summarize the effects of glutamine supplementation on endogenous glutamine metabolism. From the data it is evident that endogenous glutamine metabolism reacts differently depending on the pathophysiologic condition of the organism. Parenterally supplemented glutamine, often but not always results in increased glutamine plasma concentrations. However, this does not automatically translate into enhanced tissue glutamine levels. No clear effects have been demonstrated on whole body protein metabolism. Endogenous glutamine Ra remains virtually unchanged during glutamine administration, thereby increasing the amount of glutamine available for consumption. However, glutamine metabolism in the individual organs is affected differently and the contribution of these organs to interorgan glutamine metabolism also alters due to exogenous glutamine infusion as shown in figure 7.
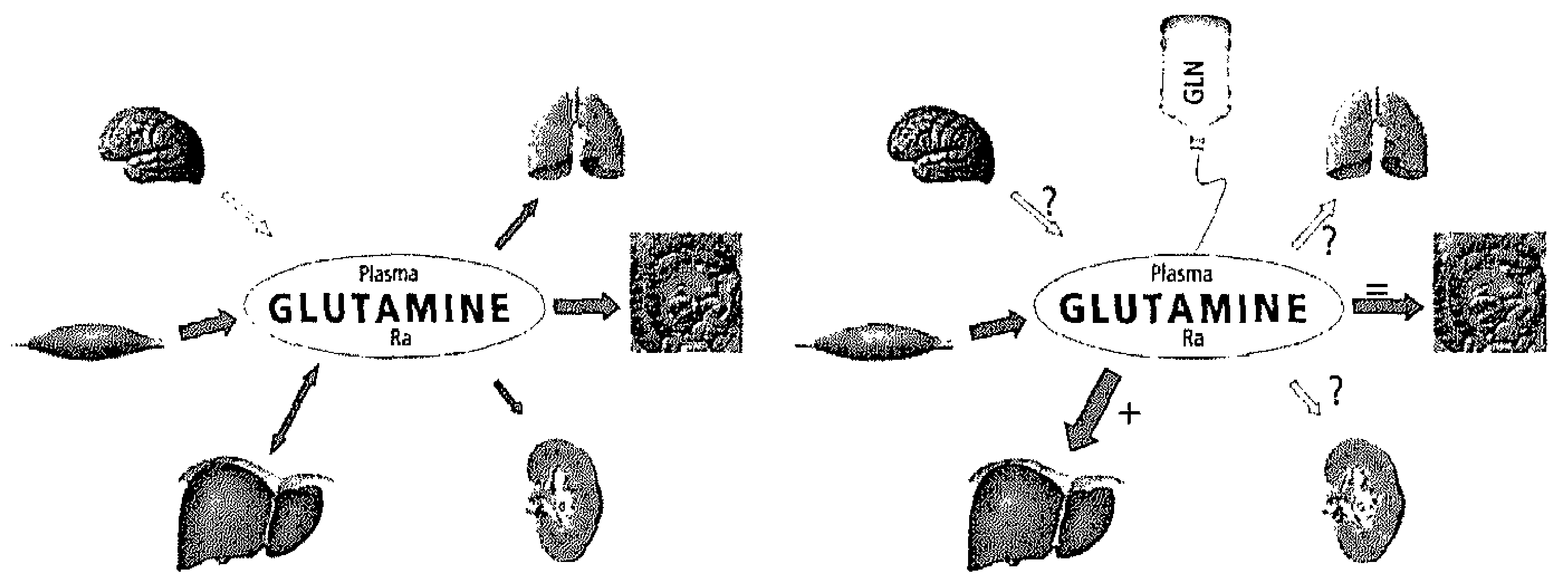

Figure $7 \quad$ Relative contributions of the main organs involved in interorgan glutamine metabolism under basal conditions and the effects of administration of glutamine on these contributions in non-catabolic circumstances. The arrows indicate the normal net flux of glutamine (i.e. uptake or releasel from the organ to the plasma compartment. Ra means rate of appearance. ? indicates that the effects of glutamine supplementation are unknown, + indicates an increase and = no change in flux.

In muscle glutamine supplementation at best results in maintenance of intracellular glutamine concentrations. These possibly depend more on the intensity of the catabolic insult. Furthermore, net protein anabolism can be reached in control situations, but under catabolic 
conditions the maximal effect is attenuation of protein catabolism. Despite possible decrements in GS activities, glutamine efflux from muscle is not affected by glutamine administration.

In the intestine no evident effects are seen on glutamine concentrations. However, specifically in catabolic conditions, GA activity increases resulting in increased glutamine consumption rates. Glutamine supplementation stimulates glutamine uptake by the PDV when provided as a free amino acid solution, but not when added to total parenteral nutrition (as a dipeptide). Furthermore, glutamine supplementation results in an improved protein balance in the gut.

The liver may play an important role in maintaining glutamine homeostasis during exogenous glutamine supplementation by taking up and degrading the excess glutamine. Glutamine possibly results in lower GS activities while simultaneously glutamine uptake is enhanced.

Little is known with regard to the role of the kidney. Most likely, the form in which glutamine is administered (free or as a dipeptide) affects its renal metabolism.

Although an essential nutrient for the immune system, even less is known with regard to effects of glutamine supplementation on its metabolism by the immune cells.

Unfortunately, many aspects of changes induced in endogenous glutamine metabolism by glutamine supplementation still remain to be clarified in order to understand why and when glutamine supplementation is useful in clinical practice. New techniques such as applied in proteomics may help clarify the diverse actions of glutamine during basal and stressed conditions 386 . 


\section{REFERENCES}

1. Hlasiwetz H, Habermann J. Ueber die proteinstoffe. Ann Chem. 1873:109.

2. Rose WC. The nutritive significance of the amino acids. Physiol Rev! 1938:18:100-130.

3. Eagle $\mathrm{H}$, Oyama VI, Levy $M$. The growth response of mammalian cells in tissue culure to l. glutamin: and L-glutamic acid. J Biol Chem. 1955;218:607.017.

4. Eagle H. Nutrition needs of mammalian cells in tissue cultures. Science. 1455;: 22:501504.

5. Windmueller HG. Glutamine utilization by the small intestine. Adv Enamol 1082:201 237.

6. Windmueller $\mathrm{HG}$, Spaeth $\mathrm{AE}$. Uptake and metabolism of plasma glutamine by the small intestine. The journal of biological chemistry. 1974;249:5070-5070.

7. Windmueller $\mathrm{HG}$, Spaeth AE. Respiratory fuels and nitrogen metabolism in vivo in small intestine of rats. The journal of biological chemistry. 1980;255:107-112.

8. Souba WW, Klimberg VS, Plumley DA, et al. The role of glutamine in maintaining a healthy gut and supporting the metabolic response to injury and infection. J Surg Res. 1490:48:383-391.

9. Lacey JM, Wilmore DW. Is glutamine a conditionally essential amino acid? Nutr Rev. 1000:48:297. 309.

10. Buchman AL. Glutamine: cornmercially essential or conditionally essentiai? A critical appraisal of the human data. Am J Clin Nutr. 2001;74:25.32.

11. Medina MA. Glutamine and cancer. I Nutr. 2001;131:2539S-2542S.

12. Young VR, Ajami AM. Glutamine: the emperor or his clothes? I Nutr. 2001:131:2449S-2450S

13. Nurjhan N, Bucci A, Periello G, et al. Glutamine: a major gluconeogenic precursor and vehicle for interorgan carbon transport in man. J Clin Invest. 1095:05:272.277.

14. Hankard RG, Haymond MW, Darmaun D. Role of glutamine as a glucose precursor in fasting humans. Diabetes. 1997;46:1535-1541.

15. Roth E, Karner J, Ollenschlager G. Glutamine: an anabolic effector? JPEN J Parenter Enteral Nutr. $1990 ; 14: 130$ S-136S.

10. Aulick $\mathrm{H}$, Wilmore DW. Increased peripheral amino acid release following injury. Surgery. 1979;85:560-565.

17. Vinnars E, Bergstrom J, Furst P. Influence of the postoperative state on the intracellular amino acids in human muscle tissue. Ann Surg. 1975;182:665-671.

18. Clowes GHA. Amino acid transfer between muscle and the visceral tissues in man during health an disease. In: Odessey R, ed. Problems and potential of branched chain amino acids in physiology and medicine: Elsevier Science Publishers; 1986:209-334.

19. Newsholme EA, Parry Billings M. Properties of glutamine release from muscle and its importance for the immune system. JPENJ Parenter Enteral Nutr. 1990;14:03S-67S.

20. Roth E, Zoch G, Schulz F, et al. Amino acid concentrations in plasma and skeletal muscle of patients with acute hemorrhagic necrotizing pancreatits. Clin Chem. 1985:31:1305-1300.

21. Newsholme P, Procopio J, Lima MM, Pithon-Curi TC, Curi R. Glutamine and glutamate - their central role in cell metabolism and function. Cell Biochem Funct. 2003;21:1-9.

22. Darmaun D, Matthews DE, Bier DM. Glutamine and glutamate kinetics in humans. Am I Physiol. 1986;251:E117-126.

23. Labow BI, Souba WW. Glutamine. World / Surg. 2000:24:1503-1513.

24. Meister A. Metabolism of glutamine. Physiol Rev. 1056;30:103-127. 
25. van Acker BA, von Meyenfeld MF, van der Hulst KR, et al. Glutamine: the pivot of our nitrogen economy? IPEN I Parenter Enteral Nutr. 1904:23:54548.

20. Rennie M. Hundal HS, Babij B, et al. Characteristics of a glutamine carrier in skeletat muscle have important consequences for nitrogen loss in injury, infection, and chronic disease. Lancet. 1980:2:1008-1012.

27. Jepson MM, Bates PC, Broadbent P, Pell M, Millward DI. Relationship between glutamine concentration and protein synthesis in rat skeletal muscle. Am J Phwsiol. 1988;255:E160-172.

28. MacLennan PA, Smith $\mathrm{K}$, Weryk B, Wart PW, Rennie MJ. Inhibition of protein breakdown by glutamine in perfused rat skeletal muscle. FEBS Lett. 1988;237:133-136.

29. Maclennan PA, Brown RA. Rennie MJ. A positive relationship between protein synthetic rate and intracellular glutamine concentration in perfused rat skeletal muscle. FF:BS Lett. 1987;215:187-191.

30. Zhou X. Thompson IR. Regulation of protein tumover by glutamine in heat-shocked skeletal myotubes. Biochim Biophys Acta. 1097;1357:234242.

31. Wusteman M, Flia M. Effect of glutamine infusions on glutamine concentration and protein synthetic rate in rat muscle. J Parenter Enteral Nutr. 1091;15:521-525.

32. Jepson MM, Millward DJ. Impact of glutamine infusions on muscle protein synthesis in fasted and endotoxin treated rats. Clin Nutr. 1991;10 (5uppl):43-40.

33. Fang $\mathrm{CH}$, James JH, Fischer JE, Hasselgren PO. Is muscle protein turnover regulated by intracellular glutamine during sepsis? JPEN J Parenter Enteral Nutr. 1995;10:279-285.

34. Olde Damink SW, de Blaauw I, Deutz NE. Soeters PB. Effects in vivo of decreased plasma and intracellular muscle glutamine concentration on whole-body and hindquarter protein kinetics in rats. Clin Sci /Colch/. 1909;90:639-040.

35. Mittendorfer B, Volpi E, Wolfe RR. Whole body and skeletal muscle glutamine metabolism in healthy subjects. Am J Physiol Endocrinol Metab. 2001;280:E323-333.

36. Sakurai Y, Aarsland A, Herndon DN, et al. Stimulation of muscle protein synthesis by long-term insulin infusion in severely burned children. Ann Surg. 1995:222:283.297.

37. Ferrando AA, Chinkes DL, Wolf SE, Matin S, Herndon DN, Wolfe RR. A submaximal dose of insulin promotes net skeletal muscle protein synthesis in patients with severe burns. Ann Surg. 1990;229:11 18.

38. Neu J. Glutamine in the fetus and critically ill low birth weight neonate: metabolism and mechanism of action. J Nutr. 2001;131:2585S-2589S; discussion 2590S.

39. Lemons JA, Adcock EW, Jones III MD, Naughton Jr. MA, Meschia G, Battaglia FC. Umbilical uptake of amino acids in the unstressed fetal lamb. J Clin Invest. 1976;58:1428-1434.

40. Neu J, Roig JC, Meetze WH, et al. Enteral glutamine supplementation for very low birth weight infants decreases morbidity. J Pediatr. 1947;131:091-099.

41. Lacey JM, Crouch JB, Benfell $K$, et al. The effects of glutamine-supplemented parenteral nutrition in premature infants. JPEN J Parenter Enteral Nutr. 1996;20:74-80.

42. Hankard RG, Darmaun D, Sager BK, D'Amore D, Parsons WR, Haymond M. Response of glutamine metabolism to exogenous glutamine in humans. Am J Physiol. 1995;209:E063-E070.

43. Roig JC. Shenoy VB, Chakrabarti R, Lau JYN, Neu J. Localization of rat small intestine glutamine synthetase using immunofluorescence and in situ hybridization. JPEN J Parenter Enteral Nutr. 1995;19:179.181.

44. Gebhardt R, Mecke D. Heterogeneous distribution of glutamine synthestase among rat liver parenchymal cells in situ and in primary culture. EMBO J. 1983;2:507.570. 
45. Rothstein JD, Tabakoff B. Alteration of striatal glutamate release after glutamine synthetase inhibition. J Neurochem. 1984;43:1438-1446.

40. Cooper AJL. Glutamine synthetase. In: Kvamme E, ed. Glutamine and glutamate in mammals. Vol I: CRC Press; 1988:7-31.

47. Haberle J, Gorg B, Rutsch F, et al. Congenital glutamine deficiency with glutamine synthetase mutations. N Engl J Med. 2005;353:1926-1933.

48. Ardawi MS. Glutamine-synthesizing activity in lungs of fed, starved, acidotic, diabetic, injured and septic rats. Biochem J. 1990;270:829-832.

49. Labow BI, Souba WW, Abcouwer SF. Glutamine synthetase expression in muscle is regulated by transcriptional and posttranscriptional mechanisms. Am J Physiol. 1999;276:E1136-1145.

50. Mezzaroba V, Torrent A, Leydier I, et al. The role of adrenal hormones in the response of glutamine synthetase to fasting in adult and old rats. Clin Nutr. 2003:22:569-575.

51. Labow BI, Souba WW, Abcouwer SF. Mechanisms governing the expression of the enzymes of glutamine metabolism--glutaminase and glutamine synthetase. J Nutr: 2001;131:24675-2474S.

52. Wolfe RR. Session II: physiological aspects of glutamine metabolism I - discussion summary. I Nutr. 2001;131:2496S-2497S.

53. Smith RJ, Larson S, Stred SE, Durschlag RP. Regulation of glutamine synthetase and glutaminase activities in cultured skeletal muscle cells. / Cell Physiol. 1984;120:197-203.

54. Zalkin H, Smith JL. Enzymes utilizing glutamine as an amide donor. Adv Enzymol Relat Areas Mol Biol. 1998;72:87-144.

55. McCauley R, Kong SE, Heel K, Hall JC. The role of glutaminase in the small intestine. Int J Biochem Cell Biol. 1999;31:405-413.

56. Brosnan JT, Ewart HS, Squires SA. Hormonal control of hepatic glutaminase. Advan Enzyme Regul. 1995;35:131-146.

57. Curthoys NP, Watford M. Regulation of glutaminase activity and glutamine metabolism. Annu Rev Nutr. 1995;15:133-159.

58. Souba WW. Glutamine: a key substrate for the splanchnic bed. Annu Rev Nutr: 1991;11:285-308.

59. Boza JJ, Moennoz D, Jarret AR, et al. Neither glutamine nor arginine supplementation of diets increase glutamine body stores in healthy growing rats. Clin Nutr. 2000;19:319-325.

60. Watford $M$, Smith EM, Erbelbelding EJ. The regulation of phophate-activated glutaminase activitiy and glutamine metabolism in the streptozotocin-diabetic rat. Biochem J. 1984;224:207-214.

61. Watford M, Erbelbelding EJ, Shapiro AC, Zakow AM, Smith EM. The adaptive response of phosphateactivated glutaminase in the rat. Contrib Nephrol. 1985;47:140-144.

62. Fox AD, Kripke SA, Berman JM, McGintey RM, Settle RG, Rombeau JL. Dexamethasone administration induces increased glutaminase specific activity in the jejunum and colon. / Surg Res. 1988;44:391. 396.

63. Watford $M$. Regulation of experssion of the genes for glutaminase and glutamine synthetase in the acidotic rat. Contrib Nephrol. 1991;92:211-217.

64. Kuhn KS, Schuhmann K, Stehle P, Darmaun D, Furst P. Determination of glutamine in muscle protein facilitates accurate assessment of proteolysis and de novo synthesis-derived endogenous glutamine production. Am J Clin Nutr. 1999;70:484-489.

65. Elia M, Folmer P, Schlatmann A, Goren A, Austin S. Amino acid metabolism in muscle and in the whole body before and after ingestion of a single mixed meal. Am J Clin Nutr. 1989;49:1203-1210.

66. Haussinger D. Liver glutamine metabolism. JPEN J Parenter Enteral Nutr. 1990;14:56S-62S. 
07. Lenzen C. Sobell S, Sies H, Haussinger D. pH control of hepatic glutamine degradation. Role of transport. Eur / Biochem. 1987:106:483-488.

08. Broer S. Adaptation of plasma membrane amino acid transport mechanisms to physiological demands. Pflugers Arch. 2002;444:457.400.

69. Bode BP. Recent molecular advances in mammalian glutamine transport. J Nutr. 2001;131:2475S24855 .

70. Chaudhry FA. Reimer RJ. Edwards RH. The glatamine commute: take the $\mathrm{N}$ line and transfer to the A. I Cell Biol. 2002;157:349-355.

71. Hatanaka T, Huang $W$, Wang H, et al. Primary structure, functional characteristics and tissue expression pattern of human ATA2, a subtype of amino acid transport system A. Biochim Biophys Acta. 2000;1407.

72. Chaudhry FA, Reimer RJ, Krizaj D, et al. Molecular analysis of system $\mathrm{N}$ suggests novel physiological roles in nitrogen metabolism and synaptic transmission. Cell. 1999;99:769-780.

73. Broer A, Brookes N, Ganapathy V, et al. The astroglial ASCT2 amino acid transporter as a mediator of glutamine efflux. J Neurochem. 1999;73:2184.2194.

74. Ritchie JWA. Baird FE, Christie GR, et al. Mechanisms of glutamine transport in rat adipocytes and acute regulation by cell swelling. Cell Physiol Biochem. 2001;11:250-270.

75. Herskowitz K, Bode BP, Block ER, Souba WW. The effects of endotoxin on glutamine transport by pulmonary artery endothelial cells. J Surg Res. 1991;50:356-361.

76. Souba WW, Salloum RM, Bode BP, Herskowitz K. Cytokine modulation of glutamine transport by pulmonary artery endothelial cells. Surgery. 1991;110:295.302.

77. Souba WW. Copeland EM. Cytokine modulation of brush border glutamine transport in monolayers of human Caco-2 cells. Ann Surg. 1992;215:536-545.

78. Ahmed A, Maxwell DL, Taylort PM, Rennie MJ. Glutamine transport in human skeletal muscle. Am J Physiol. 1993;264:E993-E1000.

79. Rennie MJ, Ahmed A, Khogali SE. Low SY, Hundal HS, Taylor PM. Glutamine metabolism and transport in skeletal muscle and heart and their clinical relevance. J Nutr. 1996;126:1142S-1149S.

80. Darmaun D, Weich S, Rini A, Sager BK, Altomare A, Haymond MW. Phenylbutyrate-induced glutamine depletion in humans: effect on leucine metabolism. Am J Physiol. 1998;274:E801-807.

81. Grill V, Bjorkman O, Gutniak M, Lindqvist $M$. Brain uptake and release of amino acid in nondiabetic and insulin-dependent diabetic subjects: important role of glutamine release for nitrogen balance. Metabolism. 1992;41:28-32.

82. Elia $M$. The inter-organ flux of sbstrates in fed and fasted man, as indicated by arterio-venous balance studies. Nutrition research review's. 1991;4:3-31.

83. Brosnan JT. Interorgan amino acid transport and its regulation. J Nutr. 2003;133:2068S-2072S.

84. Van Acker BAC, Hulsewé KWE, Wagenmakers AJM, et al. Absence of glutamine isotopic steady state: implications for the assessment of whole-body glutamine production rate. Clin Sci (Colch). 1998;95:339-340.

85. Barrett EJ, Revkin JH, Young LH, Zaret BL, Jacob R, Gelfand RA. An isotopic method for measurement of muscle protein synthesis and degradation in vivo. Biochem J. 1987;245:223-228.

80. Biolo G, Fleming RYD, Maggi SP, Wolfe RR. Transmembrane transport and intracelullar kinetics of amino acids in human skeletal muscle. Am / Physiol. 1995;268:E75-E84.

87. Wagenmakers AJ. Tracers to investigate protein and amino acid metabolism in human subjects. Proc Nutr Soc. 1999:58:987-1000. 
88. Blaauw de I, Deutz NEP, von Meyenfeldt MF. Increased whole-body protein and glutamine turnover in advanced cancer is not matched by an increased muscle protein and glutamine turnover. I Surg Res. 1997;68.

89. Blaauw de I, Deutz NEP, von Meyenfeldt MF. Cancer reduces the metabolic response of muscle to surgical stress in the rat. J Surg Res. 1998;80.

90. Watford $\mathrm{M}$. Is there a requirement for glutamine catabolism in the small intestine? $\mathrm{Br} J$ Nutr. 1999;81:261-262.

91. Gate JJ, Parker DS, Lobley GE. The metabolic fate of the amido-N group of glutamine in the tissues of the gastrointestinal tract in 24h-fasted sheep. Br J Nutr. 1999;81:297-306.

92. Gerich JE, Meyer C, Stumvoll MW. Hormonal control of renal and systemic glutamine metabolism. $J$ Nutr. 2000;130:995S-100 IS.

93. Hiscock N, Pedersen BK. Exercise-induced immunodepression- plasma glutamine is not the link. $J$ Appl Physiol. 2002;93:813-822.

94. Owen OE, Smalley KJ, D'Alessio DA, Mozzoli MA, Dawson EK. Protein, fat, and carbohydrate requirements during starvation: anaplerosis and cataplerosis. Am J Clin Nutr. 1998;08:12-34.

95. Hankard RG, Haymond MW, Darmaun D. Role of glucose in the regulation of glutamine metabolism in health and in type 1 insulin-dependent diabetes. Am J Physiol Endocrinol Metab. 2000;279:E608-613.

90. Cynober LA. Plasma amino acid levels with a note on membrane transport: characteristics, regulation, and metabolic significance. Nutrition. 2002;18:761-766.

97. Meyer C, Dostou J, Nadkarni V, Gerich JE. Effects of physiological hyperinsulinemia on systemic, renal and hepatic substrate metabolism. Am J Physiol. 1998;275:F915-F921.

98. Biolo G, Fleming RY, Maggi SP, Nguyen TT, Herndon DN, Wolfe RR. Inhibition of muscle glutamine formation in hypercatabolic patients. Clin Sci (Lond). 2000;99:189-194.

99. Cunningham JN, Jr., Carter NW, Rector FC, Jr., Seldin DW. Resting transmembrane potential difference of skeletal muscle in normal subjects and severely ill patients. J Clin Invest. 1971;50:49-59.

100. Tracey KJ, Lowry SF, Beutler B, Cerami A, Albert JD, Shires GT. Cachectin/tumor' necrosis factor mediates changes of skeletal muscle plasma membrane potential. J Exp Med. 1986;164:1308-1373.

101. Gibson WH, Cook JJ, Gatipon G, Moses ME. Effect of endotoxin shock on skeletal muscle cell membrane potential. Surgery. 1977;81:571-577.

102. Schlenker T, Feranchak AP, Schwake L, Stremmel W, Roman RM, Fitz JG. Functional interactions between oxidative stress, membrane $\mathrm{Na}(+)$ permeability, and cell volume in rat hepatoma cells. Gastroenterology. 2000;118:395-403.

103. Palmer TE, Griffiths RD, Jones C. Effect of parenteral L-glutamine on muscle in the very severely ill. Nutrition. 1996;12:316-320.

104. Gore DC, Wolfe RR. Glutamine supplementation fails to affect muscle protein kinetics in critically ill patients. JPEN J Parenter Enteral Nutr. 2003;26:342-349.

105. Tjader I, Rooyackers OE, Forsberg AM, Vesali RF, Garlick PJ, Wernerman J. Effects on skeletal muscle of intravenous glutamine supplementation to ICU patients. Intensive Care Med. 2004;30:266-275.

106. Blomqvist BI, Hammerqvist F, von der Decken A, Wernerman J. Glutamine and alpha-ketoglutarate prevent the decrease in muscle free glutamine concentration and influence protein synthesis after total hip replacement. Metabolism. 1995;44:1215-1222.

107. Hammarqvist F, Wernerman J, von der Decken A, Vinnars E. Alanyl-glutamine counteracts the depletion of free glutamine and the postoperative decline in protein synthesis in skeletal muscle. Ann Surg. 1990;212:637-644. 


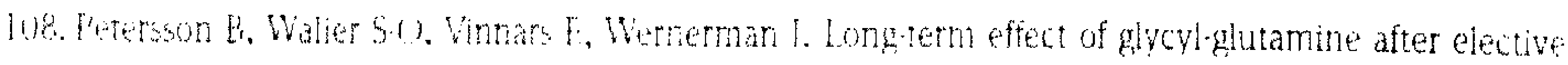

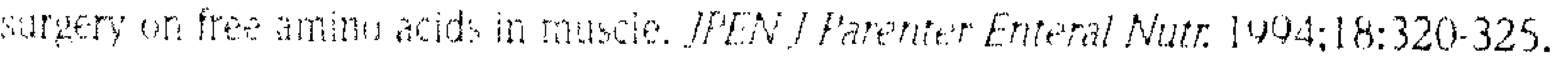

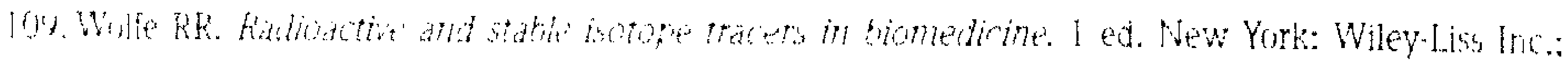
1012.

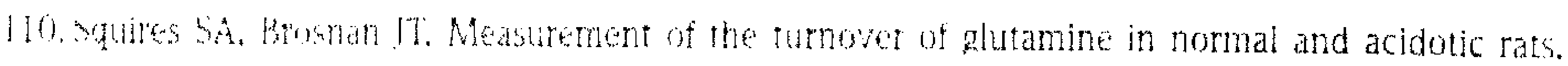
bischen i. 10832102277280

111. Habeth AE, Bheame N, Messina AB, et al Regulation of hepatic glutamine metabolism during exercise in the dog. Am / Fhrsiol. 1008;275:E055-0\%4.

112. Hatmam RN, Bergman EN. Chutamine metabolism, interotgan transport, and glucogenicity in the

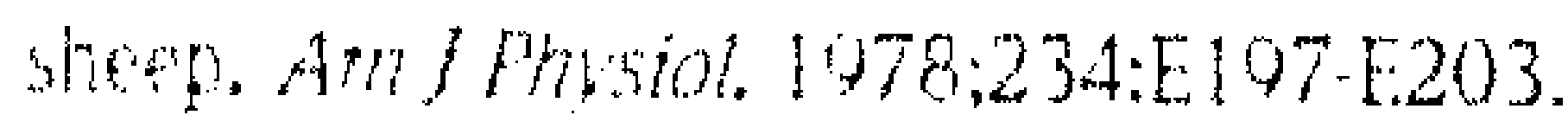

113. Nutu R, (Obitsu T, Fernandez Cuintela A, al, Glutamine metabolism in ovine splanchnic tissues: offects of iniusicu of ammonium bicarbonate or aminn acids in the abomasum. Br I Nutr. 2002;87:357. 3 tits.

114. Keider mE, Sturnoll M, Mever C, (Wverkamp D. Welle S, Gerich I. Steady state and non-steady state measurements of plasma glutamine tumover in humans. Am / Physiol. 1997;272:E021-Eo27.

115. Marthews DE. Campbell RO. The effect of dietary protein intake on glutamine and glutamate nitrogen metabolism in humans. Am I Clin Nutr. 1002:55:003070.

110. Gore DC. Jatoor $\mathrm{F}$. Glutamine kinetics in burn patients. Comparison with hormonally induced stress in volunteers. Arch.Surg. 1994:129:1318.1323.

17. Darmaun D, Dechelotte P. Role of leucine as a precursor of glutamine alpha-amino nitrogen in vivo in humans. Am J Phisiol. 1991:200:E320-329.

118. Marthews DE. Marano MA, Campbell RG. Splanchnic bed utilization of glutamine and glutamic acid in humans. Am J Ptysiol. 1993:204:E848-E854.

119. Haisch $M$, Fukagawa Nk, Matthews DE. Oxidation of glutamine by the splanchnic bed in humans. Am J Physiol Endocrinol Metab. 2000:278:E593.602.

120. Darmaun D, Matthews DE, Bier DM. Physiolagical hypercortisolemia increases proteolysis, glutamine, and alanine production. Am J Physiol. 1988;255:E300-373.

121. Williams BD, Chinkes DL, Wolfe RR. Alanine and glutamine kinetics at rest and during exercise in humans. Med Sci Sports Exerc. 1998;30:1053-1058.

122. lackson NC. Carroll PV, Russell-Jones DL, Sonksen PH, Treacher DF, Umpleby AM. The metabolic consequences of critical illness: acute effects on glutamine and protein metabolism. Am J Physiol. 1909:270:5103-170.

123. Stumvoll MW, Perriello G. Nurihan N, et al. Glutamine and alanine metabolism in NIDDM. Diabetes. $1906 ; 4: 803 \cdot 808$.

124. Carbonnel F, Messing B, Darmaun D, et al. Energy and protein metabolism in malnutrition due to nonneoplastic gastrointestinal diseases. Metabolism. 1905;44:1110-1115.

125. Parimi PS, Devapatla S, Gruca LL, O'Brien AM, Hanson RW, Kalhan SC. Glutamine and leucine nitrogen kinetics and their relation to urea nitrogen in newborn infants. Am J Physiol. 2001;282:E018 E625.

120. Stumvoll MW, Meyer C, Perriello G, Kreider M, Welle S, Gerich JE. Human kidney and liver gluconeogenesis: evidence for organ substrate selectivity. Am J Physiol. 1998;274:E817 E820.

127. Parimi PS, Devapatla S, Gruca L..., Amini SB, Hanson RW, Kalhan SC. Effect of enteral glutamine or glycine on whole-body nitrogen kinetics in very-low-birth weight infants. Am / Clin Nutr. 2004;79:402 409. 
128. Stumvoll MW, Meyer C, Kreider M, Perriello G, Gerich JE. Effects of glucagon on on renal and hepatic glutamine gluconeogenesis in normal postabsorptive humans. Metabolism. 1998;47:1227-1232.

129. Mauras N, Haymond MW, Darmaun D, Vieira NE, Abrams SA, Yergey AL. Calcium and protein kinetics in prepubertal boys. Positive effects of testosterone. J Clin Invest. 1994;93:1014-1019.

130. Claeyssens S, Bouteloup-Demange C, Gachon P, et al. Effect of enteral glutamine on ieucine, phenylalanine and glutamine metabolism in hypercortisolemic subjects. Am J Physiol. 2000;278:E817-E824.

131. Battezzati A, Brillon DJ, Matthews DE. Oxidation of glutamic acid by the splanchnic bed in humans. Am J Physiol. 1995;269:E269-E276.

132. Wolfe RR, Biolo G. Muscle glutamine production in burn patients: the physiological meaning of tracer estimates: authors' reply. Clin Sci (Colch). 2001;101:301.

133. Wagenmakers AJ. Muscle glutamine production in burn patients: the physiological meaning of tracer estimates. Clin Sci (Lond). 2001;100:299-301.

134. Chiasson J, Liljenquist J, Lacey W, Jennings A, Cherrington A. Gluconeogenesis: methodological approaches in vivo. Fed Proc. 1977;36:229-235.

135. Bourreille A, Humbert B, Maugere P, Gaimiche J.P, Darmaun D. Glutamine metabolism in Crohn's disease: a stable isotope study. Clin Nutr. 2004;23:1167-1175.

136. Chang TW, Goldberg AL. The metabolic fates of amino acids and the formation of glutamine in skeletal muscle. J Biol Chem. 1978;253:3685-3695.

137. Holecek $M$. Relation between glutamine, branched-chain amino acids, and protein metabolism. Nutrition. 2002;18:130-133.

138. Perriello $\mathrm{G}$, Jorde R, Nurjhan $\mathrm{N}$, et al. Estimation of glucose - alanine - lactate - glutamine cycles in postabsorptive humans : role of skeletal muscle. Am J Physiol. 1995;269:E443-E450.

139. Hankard RG, Haymond MW, Darmaun D. Effect of glutamine on leucine metabolism in humans. Am J Physiol. 1996;271:E748-754.

140. Perriello G, Nurihan N, Stumvoll $M$, et al. Regulation of gluconeogenesis by glutamine in normal postabsorptive humans. Am J Physiol. 1997;272:E437-445.

141. Souba WW, Herskowitz K, Salloum RM, Chen MK, Austgen TR. Gut glutamine metabolism. JPEN J Parenter Enteral Nutr. 1990;14:45S-50S.

142. Owen $O$, Reichle F, Mozzoli M, et al. Hepatic, gut, and renal substrate flux rates in patients with hepatic cirrhosis. J Clin Invest. 1981;68:240-252.

143. Felig P, Wahren J, Karl I, Cerasi E, Luft R, Kipnis DM. Glutamine and glutamate metabolism in normal and diabetic subjects. Diabetes. 1973;22:573-576.

144. Felig P, Wahren J, Lars R. Evidence for interorgan amino acid transport by red blood cells in humans. Proc Natl Acad Sci U S A. 1973;70:1775-1779.

145. Vinnars E, Bergstrom J, Furst P. Effects of starvation on plasma and muscle amino acid concentrations in normal subjects. Clin Nutr. 1987;6:S62.

146. Ruderman NB, Berger $M$. The formation of glutamine and alanine in skeletal muscle. $J$ Biol Chem. 1974;249:5500.5506.

147. Marliss EB, Aoki TT, Pozefsky T, Most AS, Cahill j, G.F. Muscle and splanchnic glutamine and glutamate metabolism in postabsorptive and starved man. / Clin Invest. 1971;50:814-817.

148. Lockwood AH, McDonald JM, Reiman RE, et al. The dynamics of ammonia metabolism in man. Effects of liver disease and hyperammonemia. J Clin Invest. 1979;03:449-460.

149. Elgadi KM, Meguid RA, Oian M, Souba WW, Abcouwer SF Cloning and analysis of unique human glutaminase isoforms generated by tissue-specific alternative splicing. Physiol Genomics. 1999;1:51-02. 


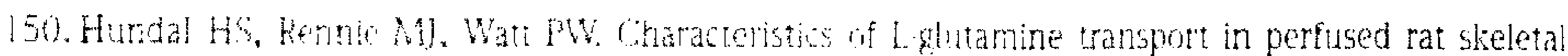

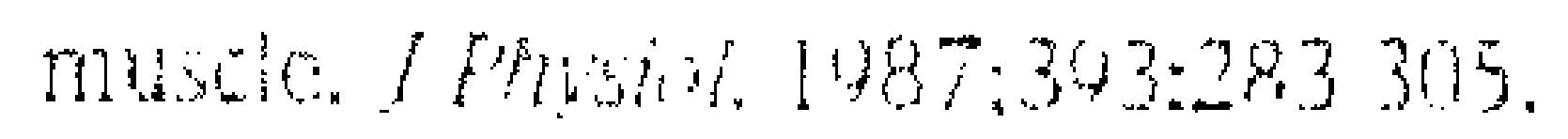

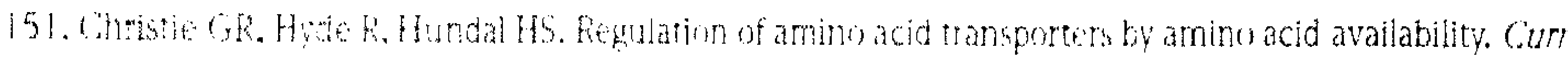
Opint cim vith neth Cith. 2001:4:425-431.

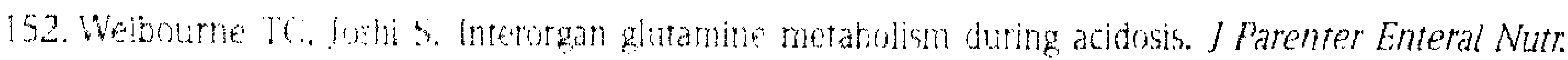
$1000: 14: 7758555$

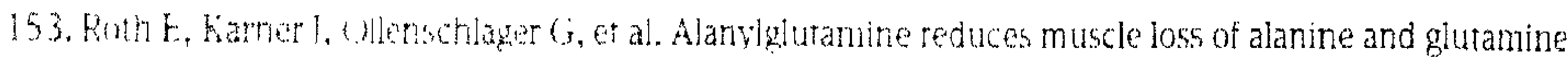

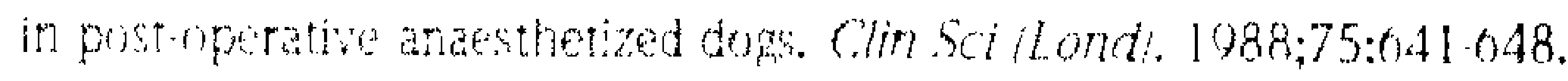

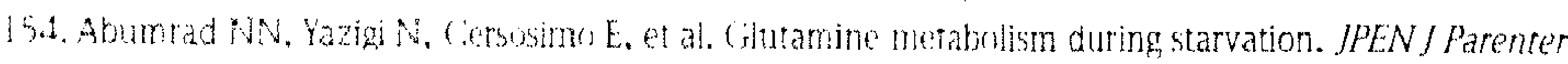
Fintoral Nitr. 100014:715705.

155. Gia M. Khan $\mathrm{K}$, Jeming $\mathrm{G}$. Fffect of mixed meal ingestion on fuel utilization in the whole body and in superficial and deep forearm tisules. Br I Nutr. 1009:81:373.381.

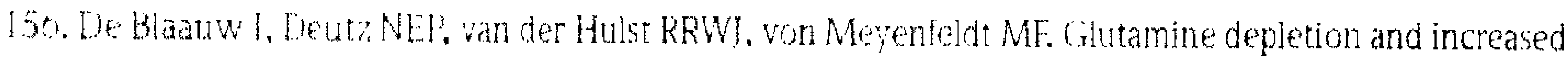
gut permeability in nonanorectic, num weight losing tumor-bearing rats. Gastroenterologs. $1007: 112: 118 \cdot 120$.

157. Babst K. Horig H, Stehle P, et al. Glutamine peptidesupplenented long, term total parenteral nutrition: stfects on intracelluar and extracellular amino acid pattems, nitrogen economy, and tissue morphology in growing rats. IFFN J Farenter Enteral Nutr. 1993:17:500.574.

158. Hulst van der RRW]. Deutz NEF, Mevenfeldt von MF, Hibers JMH, Stockbrügger RW. Decrease of mucasal glutamine concentration in the nutritionally depleted patient. Clinical Nutrition. 1994:13:228.233.

150. Ahlman B. Ijungquist O. Person B, Bindslev 1. , Wernerman J. Intestinal amino acid content in critically ill patients. IPIN J Parenter Enteral Nutr: 1905:19:272.278.

160. Shenoy V, Roig JC, Kubilis P, Neu J. Characterization of glutaminase in the developing rat small intestine. I Nutr. 1990;120:11215.1130S.

101. Tames LA. Lunn PG. Elia M. Glutamine metabolism in the gastrointestinal tract of the rat assessed by the relative activities of glutaminase (FC 3.5.1.2) and glutamine synthetase (EC 0.3.1.2). Br J Nutr. $1098 ; 70: 305-372$.

102. James LA, Lun PG. Middleton S, Elia M. Distribution of glutaminase and glutamine synthetase activities in the human gastrointestinal tract. Clin Sci /Colchl 1908;94:313-319.

103. Nagy LE, Pittler A, Krechmer N. Development of glutaminase along the villus-crypt axis in the jejunum of rat. I Pediatr Gastroetteterol Nutr. 1988:7:907-013.

164. Pinkus LM, Windmueller HG. Phosphate-dependent glutaminase of small intestine: localization and role in intestinal glutamine metabolism. Arch Biochem Biophys. 1977;182:506-517.

105. Kong SE. Hall IC, Cooper D, MCCauley RD. Starvation alters the activity and mRNA levels of glutaminase and glutamine synthestase in the rat inrestine. J Nut Biochem. 2000;11:393-400.

160. Budohoski L, Challis RA, Newshome EA. Effects of starvation on the maximal activities of some Glycolytic and citric acid-cycie enzymes and glutaminase in mucosa of the small intestine of the rat. Biochem J. 1082;206:164:172.

167. McFarlane Anderson N, Bennett H. Alleyne CA. Ammonia production by the small intestine of the rat. Biochim biophvs Actd. 1970;437:238243.

168. Muller AR, Lanyehr IM. Nalesnik MA, et al. Mucosal glutaminase activity and histology as parameters of small bowel prescrvation iniury. J Surg Res. 1004;5r:207215. 
109. Mueller AR, Nalesnik MA, Langrehr JM, et al. Evidence that small bowel preservation causes pritivit!'s basement membrane and endothelial rather than epithelial cell injury. Tranoplantation. 1 y. $3.5 \%: 14 \mathrm{dy}$ 1504.

170. Souba WW, Herskowitz K, Klimberg VS, et al. The effects of sepsis and endrioxemia ur gut giuman metabolism. Ann Surg. 1990;21 1:543-549.

171. Klimberg VS, Souba WW, Salloum RM, et al. Intesinal glutamine metabolism atter mascive small bowa resection. Am J Surg. 1990;159:27-32.

172. Salleh M, Ardawi MS, Majzoub MF, Newsholme EA. Effect of glucocorticoid treatment on glususe and glutamine metabolism by the small intestine of the rat. Clin Sci ILond 1 1088:75:03-100.

173. Zhang GX, Lai JH, Jia TW, Wang WZ, Wang IY. Effect of epidermal growth factor on plutamine: metabolic enzymes in small intestine and skeletal muscle of parenterally fed rats. Nutritim. 1997; 13:052-655.

174. Deutz NEP, Reijven PL, Athanasas G, Soeters PB. Post-operative changes in hepatic, intertimal, spleric and muscle fluxes of amino acids and ammonia in pigs. Clin Sci (Colch). 1092;83:007.014.

175. Lopez HW, Moundras C, Morand C, Demigne C, Remesy C. Opposite fluxes of glutamine and alanine in the splanchnic area are an efficient mechanism for nitrogen sparing in rats. J Nutr. 1998;128:1487.1404.

176. Souba WW, Wilmore DW. Postoperative alteration of arteriovenous exchange of amino acids acros the gastrointestinal tract. Surgery. 1983;94:342-350.

177. Souba WW, Scott TE, Wilmore DW. Intestinal consumption of intravenously administered fuels. JPEN J Parenter Enteral Nutr. 1985;9:18-22.

178. Cersosimo E, Williams PE, Radosevich PM. Hoxworth BT, Lacy WW, Aburnad NN. Role of glutamine in adaptations in nitrogen metabolism during fasting. Am J Physiol. 1980;250:E622-5028.

179. Souba WW, Smith RJ, Wilmore DW. Glutamine metabolism by the intestinal tract. JPEN J Parenter Enteral Nutr. 1985;9:608-617.

180. Haque SM, Chen K, Usui N, et al. Alanyl-glutamine dipeptide supplemented parenteral nutrition improves intestinal metabolism and prevents increased permeability in rats. Ann Surg. 1900;223:334 341.

181. Bruins MJ, Deutz NE, Soeters PB. Aspects of organ protein, amino acid and glucose metabolism in a porcine model of hypermetabolic sepsis. Clin Sci (Lond). 2003:104:127.141.

182. van der Hulst RR, von Meyenfeldt MF, Deutz NE, Soeters PB. Glutamine extraction by the git is reduced in depleted patients with gastrointestinal cancer. Ann Surg. 1997:225:112121.

183. Horvath K, Jami M, Hill ID, Papadimitriou JC, Magder LS, Chanasongcram S. Isucaloric giuramine free diet and the morphology and function of rat small intestine. JPEN J Parenter Enteral Nutn. 1996;20:128-134.

184. Reeds PJ, Burrin DG. Glutamine and the bowel. / Nutr. 2001;131:2505S.

185. Barle H, Ahlman B, Nyberg B, Andersson K, Essen P, Wernerman J. The concentrations of free amino acids in human liver tissue obtained during laparoscopic surgery. Clin Physiol. 1090;10:217.227.

180. Krebs HA. Metabolism of amino acids. IV. The synthesis of glutamine from glutamic acid and ammunia, and the enzymic hydrolysis of glutamine in animal tissues. Biochem J. 1935:20:1951-1960.

187. Remesy C, Moundras C, Morand C, Demigne C. Glutamine or glutamate release by the liver constitutes a major mechanism for nitrogen salvage. Am J Physiol. 1997:272:6257-204.

188. Haussinger D. Nitrogen metabolism in the liver: structural and functional organization and physiological relevance. Biochem J. 1990;267:281-290. 
184. Stall B. Hausinger n. Functimal hepatocyte heterogeneity. Vascular 20xoglutarate is almost

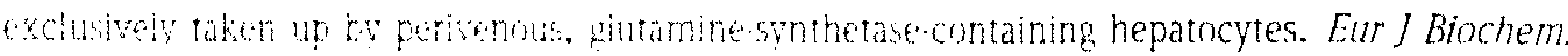
10891810704760

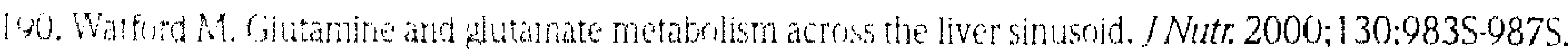

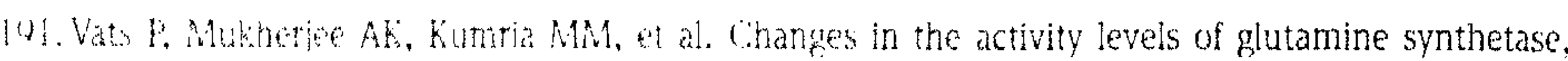
piutaminate and glycogen sunthetase in rats subjected to hypoxic stress. Int I Biometeorol 1040.4220205201 .

142. Nisim I, Brosman MF, Yudkroff M, Nissmin I. Brosnan JT. Studies of hepatic glutamine metabolism in the perfused rat liver with 15N-latoled glutamine. I Riol Chem. 1009;274:28958-28965.

103. Brosnan IT. Brisnan MF. Hepatic gilluminase a special role in urea synthesis? Nutrition. 2002;18:455. $45 \%$

194. Stewart FM, Walser M. Short term regulation of ureagenesis. J Biol Chem. 1980;255:5270-5280.

195. Watfrd M. Heparic gluaminase expression: telationship to kidney-rype glutaminase and to the urea cycle. Fato /. 1903:7:1408-1474.

190. Yang D, Hazey WW, David $\mathrm{F}$ et al. Integrative physiology of splanchnic glutamine and ammonium metatbolism. Am I Fhysiol Endoctinol Metab. 2000;278:E4n9-476.

197. Low SY, Salter M, knowles RG. Pogson I, Rennie MJ. A quantitative analysis of the control of glutanine catabolism in rat liver cells. Biochem l. 1903;205:017-624.

198. Haussinger D, koth E, I.ang F. Gerok. W. Celyular hydration state: an important determinant of protein catabolism in health and disease. The Lancet. 1993;341:1330-1332.

109. Remesy C. Morand C, Demigne C. Fafoumoux P. Control of hepatic utilization of glutamine by transport processes or cellular metabolism in rats fed a high protein diet. / Nutr. 1988;118:560.578.

200. Burger HI, Gebhardt R, Mayer (, Mecke D. Different capacities for amino acid transport in periportal and perivenous hepatocytes isolated by digitonin/collagenase perfusion. Hepatology. 1989;9:22.28.

201. Felig P. Amino acid metabolism in man. Annu Rev Biochem. 1975;44:933-955.

202. Ardawi MS. Glutamine metabolism in the lungs of glucocorticoid treated rats. Clin Sci /Colch/. $1001: 81: 37-42$

203. Lukaszewicz G, Abcouwer SF. Labow BI, Souba WW. Glutamine synthetase gene expression in the lungs of endotoxin-treated and adrenalectomized rats. Am I Physiol. 1997;273:L1182-1190.

204. Abcouwer SF, Bode BP. Souba WW. Glucocorticoids regulate rat glutamine synthetase expression in a tissue specific manner. J Surg Res. 1905;50:50-b5.

205. Labow BI, Abcouwer SF, Lin CM, Souba WW. Glutamine synthetase expression in rat lung is regulated by protein stability. Am J Physiol. 1098;275:L877-880.

200. Plumley DA, Souba WW. Hautamaki RD, et al. Accelerated lung amino acid release in hyperdynamic septic surgical patients. Arch Surg. 1990;125:57.61.

207. Herskiwitz K, Plumley DA, Martin TD, Hautamaki RD, Copeland EM, Souba WW. Lung glutamine flux following open heart surgery. I Surg Res. 1901;51:82-80.

208. Newsholme P, Gordon $S$, Newsholme EA. Rates of utilization and fates of glucose, glutamine, pyruvate, fatty acids and ketone bodies by mouse macrophages. Biochem J. 1987;242:031-636.

209. Horig H, Spagnoli GC, Filgucira L, et al. Exogenous glutamine requirement is confined to late events of T cell activation. / Cell Biochem. 1993;53:343-351.

210. Wilmore DW, Shabert IK. Role of glutamine in immunologic responses, Nutrition. 1998;14:61 8-020.

211. Parry Billings M, livans I, Calder PC, Newsholme FA. Does glutamine contribute to immunusuppression after major burns? Lancet. 1000);336:523-525. 
212. Spittler A, Winkler S, Gotzinger P, et al. Influence of glutamine on the phenotype and function of human monocytes. Blood. 1995;86:1564-1569.

213. Newsholme P. Why is L-glutamine metabolism important to cells of the immune system in health, postinjury, surgery or infection? J Nutr. 2001;131:2515S-2522S.

214. Newsholme EA, Parry Billings $M$. Some evidence for the existence of substrate cycles and their utility in vivo [letter]. Biochem.J. 1992;285:340-341.

215. Calder PC. Glutamine and the immune system. Clin Nutr. 1994;13:2.

210. Cassano GB, Hansson E. Uptake of $[14 \mathrm{C}]$ glutamine in the tissues of the mouse studied by whole-body autoradiography. J Neurochem. 1965;12:851-855.

217. Cersosimo E, Garlick P, Ferretti J. Renal substrate metabolism and gluconeogenesis during hypoglycemia in humans. Diabetes. 2000;49:1186-1193.

218. Battezzati A, Caumo A, Martino F, et al. Nonhepatic glucose production in humans. Am J Physiol. 2003;286:E129-E135.

219. Curthoys NP. Role of mitochondrial glutaminase in rat renal glutamine metabolism. I Nutr. $2001 ; 131: 2491 \mathrm{~S}-2495 \mathrm{~S}$

220. Burch $\mathrm{HB}$, Choi S, McCarthy WZ, Wong PY, Lowry $\mathrm{OH}$. The location of glutamine synthetase within the rat ad rabbit nephron. Biochem Biophys Res Commun. 1978;82:498-505.

221. Nishiitsutsuji-Uwo JM, Ross BD, Krebs HA. Metabolic activities of the isolated perfused rat kidney. Biochem J. 1967;103:852-862.

222. Carter P, Welbourne T. Glutamate transport regulation of renal glutaminase flux in vivo. Am J Physiol. 1997;273:E521-527.

223. Silbernagl S. Tubular reabsorption of L-glutamine studied by free-flow micropuncture and microperfusion of rat kidney. Int J Biochem. 1980;12:9-16.

224. Squires EJ, Hall DE, Brosnan JT. Arteriovenous differences for amino acids and lactate across kidneys of normal and acidotic rats. Biochem J. 1976;160:125-128.

225. Miller BM, Cersosimo E, McRae J, Williams PE, Lacey WW, Abumrad NN. Interorgan relationships of alanine and glutamine during fasting in the conscious dog. J Surg Res. 1983;35:310-318.

226. Cahill GF, Jr. Starvation in man. N Engl J Med. 1970;282:608-675.

227. Cooper AJ, McDonald JM, Gelbard AS, Gledhill RF, Duffy TE. The metabolic fate of $13 \mathrm{~N}$-labeled ammonia in rat brain. J Biol Chem. 1979;254:4982-4992.

228. Cooper AJ. Role of glutamine in cerebral nitrogen metabolism and ammonia neurotoxicity. Ment Retard Dev Disabil Res Rev. 2001;7:280-286.

229. Van Gelder NM. Metabolic interactions between neurons and astroglia: glutamine synthetase, carbonic anhydrase, and water balance. In: Jasper $\mathrm{HH}$, Van Gelder NM, eds. Basic mechanisms of neuronal hyperexcitability. New York: Alan R. Liss; 1983.

230. Behar KL, Rothman DL. In vivo nuclear magnetic resonance studies of glutamate-gamma-aminobutyric acid - glutamine cycling in rodent and human cortex: the central role of glutamine. $J$ Nutr. 2001;131:2498S-2504S.

231. Suarez I, Bodega G, Fernandez B. Glutamine synthetase in brain: effect of ammonia. Neurochem Int. 2002;41:123.142.

232. Nelson D, Rumsey WL, Erecinska M. Glutamine catabolism by heart muscle. Properties of phosphateactivated glutaminas. Biochem J. 1992;282:559-564.

233. Khogali SE, Harper AA, Lyall JA, Rennie MJ. Effects of L-glutamine on post-ischaemic cardiac function: protection and rescue. J Moll Cell Cardiol. 1998;30:819-827. 


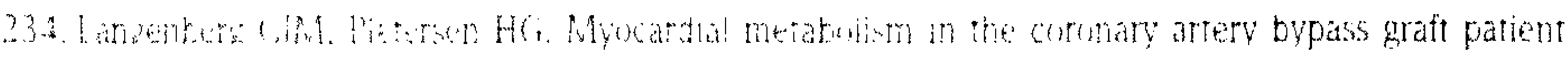

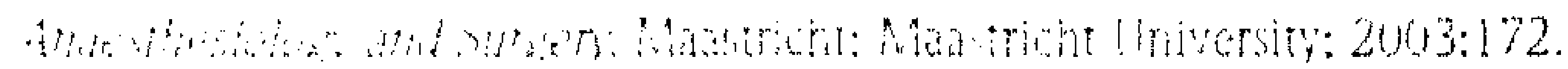

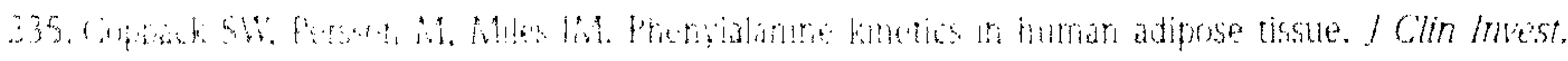

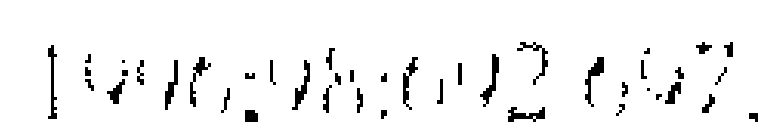

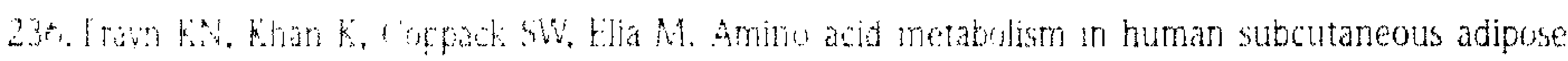

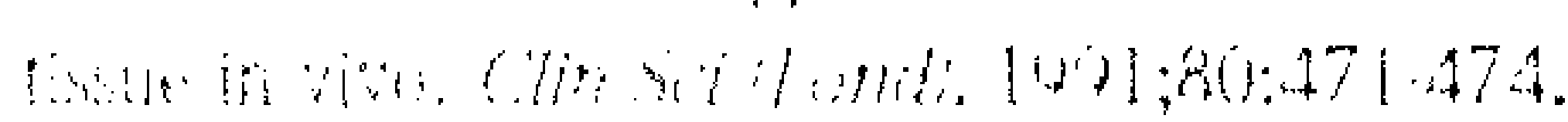

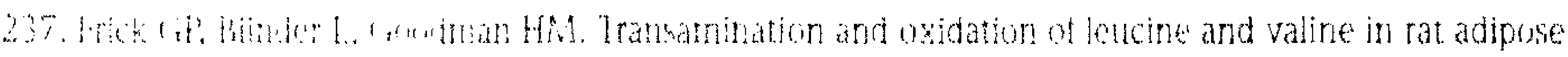

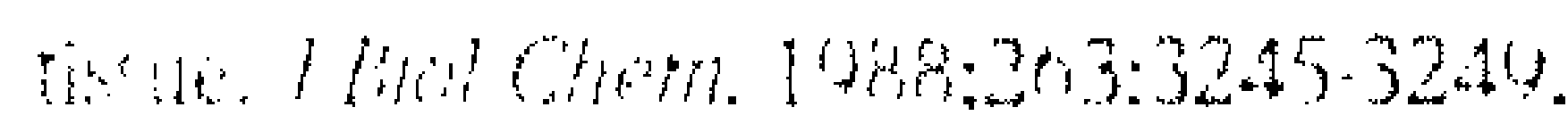

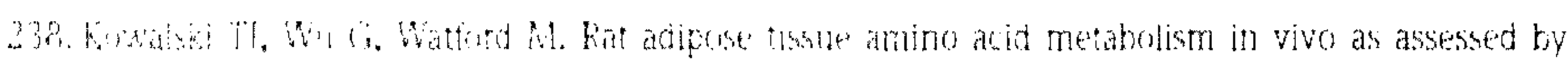

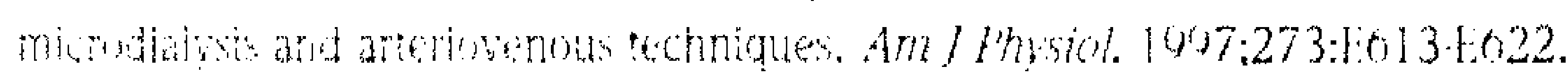

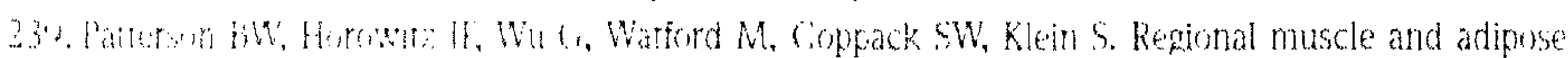

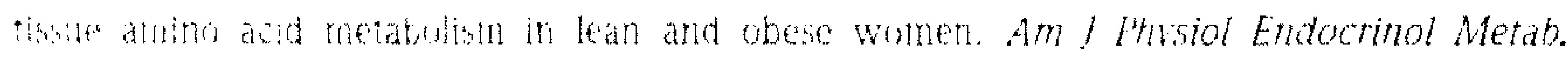
$20022825031.93 \%$

240. Barm Bilings M. Baurite RI, Lamm PM, Mortis Pl, Newholme EA. Effects of major and minor

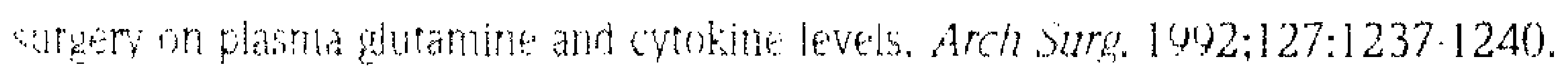

241. win Aker BAC. Hulsewe KWL, Wagenmakers AIM, Soeters PB, won Meyenfeldt MF. Clutamine

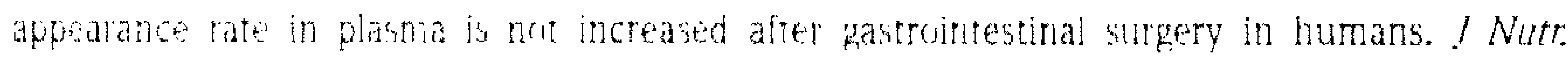
$20000 ; 130: 15 n 01571$.

242. Planche T, Dreing A. Emmerson AC, et al. Platma glutamine and glutamate concentrations in Gabonese childen with Plastnodium falciparum infection. O/ Med. 2002;05:89-97.

243. Blatuw de I. Deutz NEF. Hulsewe KWE, Meyenfeld von M: Attenuated metabolic response to surgery in tumorteaning rats. / Surgy Kes. 2003:110:371-377.

244. Hankard R, fouler (), Ricour C. Rongier M. Colomb V, Darmaun D. Glutamine metabolism in children with short-bowel syndrome: a stable isotope study. Pediatr Res. 1944;30:202-206.

245. Darmaun D, Messing B, Just B. Rongier M, Desjeux J.F. Glutamine metabolism after small intestinal resection in humans. Mrytaklism. 1991:40:42-44.

240. Deutz NEP, Deiong CHC. Athanasas G, Soeters PB. Partial enterectomy in the rat does not diminish muscle glutamine production. Metabolism. 1902:41:1343.1350.

247. Darmaun D, Rongier M, Koziet J, Robert J.J. Glutamine nitrogen kinetics in insulin-dependent diabetic humans. Am I Physiol 1901:201:E713-E718.

248. Mever C, Woerle HJ, Gerich J. Paradoxical changes of muscle glutamine release during hyperinsulinemia euglycemia and hypoglycemia in humans: further evidence for the glucose-glutamine cycte. Metabolism. 2004:53:1208-1214.

249. Yarasheski KE, Zachwieja J]. Gischler J, Crowley J, Horgan MM, Powderly WG. Increased plasma Gln and Leu Ra and inappropriately low muscle protein synthesis rate in AIDS wasting. Am J Physiol. 1048:275:E577-E583.

250. Mittendorfer B. Gore DC, Herndon DN, Wolfe RR. Accelerated glutamine synthesis in critically ill patients cannot maintain normal intramuscular free glutamine concentrations. JPEN J Parenter Enteral Nutr. 1099:23:243-250.

251. Parimi PS, Kadrofske MM, Cruca L.L, Hanson KW, Kalhan SC. Amino acids, glutamine, and protein merabolism in very luw birth weight infants. Pediatr hes. 2005:58:1259-1264.

252. Brillon DI, Theng B, Campbell $\mathrm{k}$ (, , Mathews DE. Effect of cortisol om energy expenditure and amino acid metabolism in humans. Anl I Physiol. 1905;208:1501 15513. 
253. Askanazi I, Carpentier YA, Michelsen CB, et al. Muscle and plasma amino acids following injury. Influence of intercurrent infection. Ann Surg. 1980;192:78-85.

254. Roth E, Funovics J, Muhlbacher F, Schemper M, Sporn P, Fritsch A. Metabolic disorders in severe abdominal sepsis: glutamine deficiency in skeletal muscle. Clin Nutr. 1982;1:25-41.

255. Humbert B, Le Bacquer O, Nguyen P, Dumon H, Darmaun D. Protein restriction and dexamethasone as a model of protein hypercatabolism in dogs: effect of glutamine on leucine turnover. Metabolism. 2001;50:293-298.

250. Max SR, Mill J, Mearow K, et al. Dexamethasone regulates glutamine synthetase expression in rat skeletal muscles. Am J Physiol. 1988;255:E397-E402.

257. Ardawi MS, Majzoub MF. glutamine metabolism in skeletal muscle of septic rats. Metabolism. 1991;40:155-164.

258. Austgen TR, Chakrabarti R, Chen MK, Souba WW. Adaptive regulation in skeletal muscle glutamine metabolism in endotoxin-treated rats. J Trauma. 1992;32:600-606.

259. Hundal HS, Babij P, Watt PW, Ward MR, Rennie MJ. Glutamine transport and metabolism in denervated rat skeletal muscle. Am J Physiol. 1990;259:E148-154.

260. Feng $B$, Konagaya $M$, Konagaya $Y$, et al. Neural control of glutamine synthetase activity in rat skeletal muscles. Am J Physiol. 1990;258:E757-E701.

201. O'Leary MJ, Ferguson CN, Rennie MJ, Hinds CJ, Coakley JH, Preedy VR. Sequential changes in in vivo muscle and liver protein synthesis and plasma and tissue glutamine levels in sepsis in the rat. Clin Sci (Lond). 2001;101:295-304.

262. Clowes GHA, Randall HT, Cha C-J. Amino acid and energy metabolism in septic and traumatized patients. J Parenter Enteral Nutr: 1980;4:195-205.

263. Vesali RF, Klaude M, Rooyackers OE, I TJ, Barle H, Wernerman J. Longitudinal pattern of glutamine/glutamate balance across the leg in long-stay intensive care unit patients. Clin Nutr: 2002;21:505-514.

264. Ahlman B, Andersson K, Ljungqvist O, Persson B, Wernerman J. Elective abdominal operations alter the free amino acid content of the human intestinal mucosa. Eur J Surg. 1995;101:593-601.

265. Kong S, Hall JC, Cooper D, McCauley RD. Starvation alters the activity and mRNA level of glutaminase and glutamine synthetase in the rat intestine. 2000;1 1:393-400.

266. Austgen TR, Chen MK, Flynn TC, Souba WW. The effects of endotoxin on the splanchnic metabolism of glutamine and related substrates. J Trauma. 1991;31:742.751.

267. Haque SM, Chen $\mathrm{K}$, Usui $\mathrm{N}$, et al. Effects of endotoxin on intestinal hemodynamics, glutamine metabolism, and function. Surg Today. 1997;27:500-505.

268. Salloum RM, Copeland EM, Souba WW. Brush border transport of glutamine and other substrates during sepsis and endotoxemia. Ann Surg. 1991;213:401-409.

209. Dudrick PS, Copeland EM, Bland KI, Souba WW. The early response of the jejunal brush border glutamine transporter to endotoxemia. J Surg Res. 1992;52:372-377.

270. Ardawi MS, Majzoub MF, Kateilah SM, Newsholme EA. Maximal activity of phosphate-dependent glutaminase and glutamine metabolism in septic rats. I Lab Clin Med. 1991;118:26-32.

271. Noguchi Y, James JH, Fischer JE, Hasselgren PO. Increased glutamine consumption in small intestine epithelial cells during sepsis in rats. Am J Surg. 1996;172:199-205.

272. von Allmen D, Hasselgren PO, Higashiguchi T, Frederick J, Zamir O, Fischer JE. Increased intestinal protein synthesis during sepsis and following the administration of tumour necrosis factor alpha or interleukin-1 alpha. Biochem J. 1992;286:585-589. 
273. Plauth M, Raible A, Vieillard-Baron D, Bauder-Gross D, Hartmann F. Is glutamine essential for the maintenance of intestinal function? A study in the isolated perfused rat small intestine. Int J Colorectal Dis. 1999;1 14:86-94.

274. Austgen TR, Chen MK, Dudrick PS, Copeland EM, Souba WW. Cytokine regulation of intestinal glutamine utilization. Am J Surg. 1992;163:174-179.

275. McAnena OJ, Moore FA, Moore EE, Jones TN, Parsons P. Selective uptake of glutamine in the gastrointestinal tract: confirmation in a human study. Br J Surg. 1991;78:480-482.

276. Higashiguchi T, Noguchi Y, Meyer T, Fischer JE, Hasselgren P-O. Protein synthesis in isolated enterocytes from septic or endotoxaemic rats: regulation by glutamine. Clinical Science. 1995;89:311-319.

277. Karinch AM, Pan M, Lin CM, Strange R, Souba WW. Glutamine metabolism in sepsis and infection. J Nutr. 2001;131:2535S-2538S.

278. Chen MK, Salloum RM, Austgen TR, et al. Tumor regulation of hepatic glutamine metabolism. JPEN J Parenter Enteral Nutr: 1991;15:159-164.

279. de Blaauw I, Deutz NEP, Boers W, Von Meyenfeldt MF. Hepatic amino acid and protein metabolism in non-anorectic, moderately cachectic tumor-bearing rats. J Hepatol. 1997;26:390-408.

280. Inoue Y, Pacitti AJ, Souba WW. Endotoxin increases hepatic glutamine transport activity. I Surg Res. 1993;54:393-400.

281. Fischer CP, Bode BP, Souba WW. Starvation and endotoxin act independently adn synergistically to coordinate hepatic glutamine transport. J Trauma. 1996;40:688-692.

282. Pacitti AJ, Inoue Y, Souba WW. Tumor necrosis factor stimulates amino acid transport in plasma membrane vesicles from rat liver. J Clin Invest. 1993;91:474-483.

283. Inoue $\mathrm{Y}$, Bode BP, Souba WW. Antibody to tumor necrosis factor attenuates endotoxin-stimulated amino acid transport in rat liver. Surgery. 1994;110:350-305.

284. Fischer CP, Bode BP, Takahashi K, Tanabe KK, Souba WW. Glucocorticold-dependent induction of interleukin- 6 receptor expression in human hepatocytes facilitates interleukin- 6 stimulation of amino acid transport. Ann Surg. 1996;223:610-618.

285. Pacitti AJ, Austgen TR, Souba WW. Mechanisms of increased hepatic glutamine uptake in the endotoxin-treated rat. $J$ Surg Res. 1992;53:298-305.

286. Ewart HS, Oian D, Brosnan JT. Activation of hepatic glutaminase in the endotoxin-treated rat. J Surg Res. 1995;59:245-249.

287. Nissim I. Newer aspects of glutamine/glutamate metabolism: the role of acute pH changes. Am J Physiol. 1999;277;F493-F497.

288. Hutsewe KW, Deutz NE, de Blaauw I, van der Hulst RR, von Meyenfeldt MM, Soeters PB. Liver protein and glutamine metabolism during cachexia. Proc Nutr Soc. 1997;56:801-806.

289. Blackshear PJ, Holloway PA, Alberti KG. Factors regulating amino acid release from extrasplanchnic tissues in the rat. Interactions of alanine and glutamine. Blochem J. 1975;150:379-387.

290. van de Poll MCG, Soeters PB, Deutz NEP. Renal metabolism of amino acids: its role in interorgan amino acid exchange. Am J Clin Nutr: 2004;79:185-197.

291. Souba WW, Plumley DA, Salloum RM, Copeland EM. Effects of glucocorticoids on lung glutamine and alanine metabolism. Surgery. 1990;108:213-218; discussion 218-219.

292. Welbourne TC. Interorgan glutamine flow in metabolic acidosis. Am J Physiol. 1987;253:F1069-1076.

293. Hulsewe KWE, van der Hulst RRWJ, Ramsay G, van Berlo CL, Deutz NEP, Soeters PB. Pulmonary glutamine production: effects of sepsis and pulmonary infiltrates. Intensive Care Med. 2003;29:18331830. 
294. Pan M, Masatumi W, Ryan U, Souba W. Inhibition of pulmonary microvascular endothelial glutamine transport by glucocorticoids and endotoxin. JPEN J Parenter Enteral Nutr. 1995;19:477-481.

295. Berlo van CLH, Hulst van der RRWJ, Maessen JG, et al. Lung glutamine metabolism: effects of starvation, parenteral and enteral nutrition. A study in man. Clinical Nutrition. 1995;15:86-88.

290. Johnson DJ, Brooks DC, Pressler VM, et al. Hypothermic anesthesia attenuates postoperative proteolysis. Ann Surg. 1986;204:419-429.

297. Souba WW, Smith RJ, Wilmore DW. Effects of glucocorticoids on glutamine metabolism in visceral organs. Metabolism. 1985;34:450-456.

298. Austgen TR, Chen MK, Moore W, Souba WW. Endotoxin and renal glutamine metabolism. Arch Surg. $1991 ; 126: 23-27$.

299. Tofteng F, Hauerberg J, Hansen BA, Pedersen CB, Jorgensen L, Larsen FS. Persistent arterial hyperammonemia increases the concentration of glutamine and alanine in the brain and correlates with intracranial pressure in patients with fulminant hepatic failure. I Cereb Blood Flow Metab. $2000 ; 20: 21 \cdot 27$

300. Albrecht J, Norenberg MD. Glutamine: a Trojan horse in ammonia neurotoxicity. Hepatology. 2006;44:788-794.

301. Dechelotte P, Darmaun D, Rongier M, Hecketsweiler B, Rigal O, Desjeux J-F. Absorption and metabolic effects of enterally administered glutamine in humans. Am I Physiol. 1991;260:G677-682.

302. Furst P, Albers S, Stehle P. Glutamine-containing dipeptides in parenteral nutrition. JPEN J Parenter Enteral Nutr. 1990;14:118S-124S.

303. Abumrad NN, Morse EL, Lochs H, Williams PE, Adibi SA. Possible sources of glutamine for parenteral nutrition: impact on glutamine metabolism. Am J Physiol. 1989;257:E228-234.

304. Adibi SA. Renal assimilation of oligopeptides: physiological mechanisms and metabolic importance. Am J Physiol. 1997;272:E723-736.

305. Lochs H, Hubl W, Gasic S, Roth E, Morse EL, Adibi SA. Glycylglutamine metabolism and effects on organ balances of amino acids in postabsorptive and starved subjects. AmJ Physiol. 1992;262:E155.160.

306. Lochs H, Roth E, Gasic S, Hubl W, Morse EL, Adibi SA. Splanchnic, renal, and muscle clearance of alanylglutamine in man and organ fluxes of alanine and glutamine when infused in free and peptide forms. Metabolism. 1990;39:833-830.

307. Lochs $\mathrm{H}, \mathrm{Hubl}$ W. Metabolic basis for selecting glutamine-containing substrates for parenteral nutrition. JPEN J Parenter Enteral Nutr: 1990;14:1 14\$-117S.

308. Babst R, Hörig H, Brand O, et al. Glutamine peptide-supplemented long-term total parenteral nutrition: effects on intracellular and extracellular amino acid patterns, nitrogen economy, and tissue morphology in growing rats. JPEN J Parenter Enteral Nutr: 1993;17:506-574.

309. Cersosimo E, Williams PE, Hoxworth BT, Lacy WW, Abumrad NN. Glutamine blocks lipolysis and ketogenesis of fasting. Am J Physiol. 1986;250:E248-E252.

310. Naka $S$, Saito $H$, Hashiguchi $Y$, et al. Alanylglutamine-enriched total parenteral nutrition improves protein metabolism more than branched chain amino acid-enriched total parenteral nutrition in protracted peritonitis. J Trauma. 1997;42:183-190.

311. Inoue Y, Grant JP, Snyder PJ. Effect of glutamine-supplemented total parenteral nutrition on recovery of the small intestine after starvation atrophy. JPEN J Parenter Enteral Nutr. 1993;17:165-170.

312. Zhou YP, Jiang ZM, Sun YH, Wang XR, Ma EL, Wilmore DW. The effect of supplemental enteral glutamine on plasma levels, gut function, and outcome in severe burns: a randomized, double-blind controlled clinical trial. JPEN J Parenter Enteral Nutr. 2003;27:241-245. 


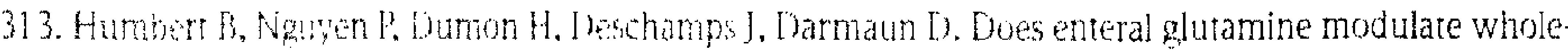

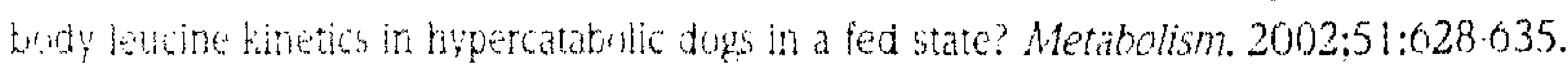

314. Shizha 1, Vaupungutum S. Kido Y. Kinh K. comparative effect of intravenously or intragastrically administred glumme ch smatl intestinal function of the rat. Tokushima.J. Exp.Med. 1990;37:49-57.

315. Hong RW, Helton WS. Ruunds 10. Wilmore DW. Gluaminesupplemented TPN preservers hepatic whuthione and improves survial following chemotherapy. Surgical Forum 1900;41:9-11.

317. Bichnan AL, Moukarzel AA, Bhuta S, ct al. Parenteral nutrition is associated with intestinal morpholugic and tunctional changes in humans. JPEN J Parenter Enteral Nutr. 1995;19:453-460.

317. Long C.L., Borghes L., Stahl R, et al. Impact of enteral feeding of a glutamine supplemented formula on the hyporaminacidemic response in trauma patients. J.Trama. 1990;40:97-102.

318. Fensen (6.. Miller RH. Tatlabiska DC. Fish I, Gianferente L. A double blind, prospective, randomized study uf glutamine enriched compared with standard peptide based feeding in critically ill patients. Am Jolin Nutr. 1006:04:015-021.

319. Freicer I.C, Peres. Bota D, Fisendrath P, Vincent $J$ L, van Gossum A. Gut mucosal and plasma concentration of glutamine: a comparison between two enriched enteral feeding solutions in critically ill patients. Nutrition Joumal. 2003;2:13.

320. Lin MT, Kung SP, Yeh SL, et al. The effect of glutamine supplemented total parenteral nutrition on nitrugen economy depends on severity of diseases in surgical patients. Clin Nutr. 2002;21:213-218.

321. Ziegler TR, Benfell K, Smith RJ, et al. Safety and metabolic effects of L-glutamine administration in humans. JPEN J Parenter Enteral Nutr. 1900;14:1375.146S.

322. Low DK, Benfell K, Smith Rl, et al. Safety of glutamine-enriched parenteral nutrient solutions in humans. Am J Clin Nutr. 1990;52:1101-1100.

323. Stehle $\mathrm{P}$, Zander J, Mertes $\mathrm{N}$, et al. liffect of parenteral glutamine peptide supplements on muscle glutamine loss and nitrogen balance after major surgery. Lancet. 1989:333:231-233.

324. Hammarqvist F, Wernerman J, Ali R, von der Decken A, Vinnars E. Addition of giutamine to total parenteral nutrition after elective abdominal surgery spares free glutamine in muscle, counteracts the fall in muscle protein synthesis, and improves nitrogen balance. Ann Surg. 1989;209:455-461.

325. Hammarqvist F, Sandgren A, Andersson $\mathrm{K}$, et al. Growth hormone together with glutamine-containing total parenteral nutrition maintains muscle glutamine levels and results in a less negative nitrogen balance after surgical trauma. Surgery. 2001;129:576-580.

320. Morlion B], Stehle P, Wachtler P, et al. Total parenteral nutrition with glutamine dipeptide after major abdominal surgery: a randomized, double-blind, controlled study. Ann Surg. 1998;227:302-308.

327. Mertes N, Schulzki C, Goeters C, et al. Cost containment through L-alanyl-L-glutamine supplemented total parenteral nutrition after major abdominal surgery: a prospective randomized double-blind controlled study. Clin Nutr. 2000;14:395-401.

328. Jiang ZM, Cao JD, Zhu XG, er al. The impact of alanyl-glutamine on clinical safety, nitrogen balance, intestinal permeability, and clinical outcome in postoperative patients: a randomized, double blind, controlled study of 120 patients. JPEN J Parenter Enteral Nutr. 1999;23:502-60.

329. van Acker BAC, Hulsewé KWE, Wagenmakers AJM, von Meyenfeldt MF, Soeters PB. Response of glutamine metabolism to glutamine-supplemented parenteral nutrition. AmJ Clin Nutr: 2000;72;790-795.

330. van Zaanen HC, van der lelie H, Timmer JG, Furst P. Sauerwein HP. Parenteral glutamine dipeptide supplementation does not ameliorate chemotherapy-induced toxicity. Cancer 1994;74:2879-2884.

331. Ciriffiths RD, Jones C, Palmer A. Six-month outcome of critically ill patients given glutaminesupplemented parenteral nutrition. Nutrition. 1997;13:295-302. 
332. Poindexter BB, Ehrenkranz RA, Stoll BJ, et al. Effect of parenteral glutamine supplementation on plasma amino acid concentrations in extremely low-birth-weight infants. Am J Clin Nutr. 2003;77:737-743.

333. Fish I, Sporay G, Beyer K, et al. A prospective randomized study of glutamine-enriched parenteral compared with enteral feeding in postoperative patients. Am J Clin Nutr. 1997;65:977-983.

334. Ziegler TR, Young LS, Benfell K, et al. Clinical and metabolic efficacy of glutamine-supplemented parenteral nutrition after bone marrow transplantation. A randomized, double-blind, controlled study. Ann Intern Med. 1992;11 6:821-828.

335. Carroll PV, Jackson NC, Russell-Jones DL, Treacher DF, Sonksen PH, Umpleby AM. Combined growth hormone/insulin-like growth factor I in addition to glutamine-supplemented TPN results in net protein anabolism in critical illness. Am J Physiol. 2004;286:E151-157.

336. van der Hulst RRWJ, van Kreel BK, Meyenfeldt von MF, et al. Glutamine and the preservation of gut integrity. Lancet. 1993;341:1363-1305.

337. Jackson NC, Carroll PV, Russell-Jones DL, Sonksen PH, Treacher DF, Umpleby AM. Effects of glutamine supplementation, GH, and IGH-I on glutamine metabolism in critically ill patients. Am J Physiol Endocrinol Metab. 2000;278:E226.233.

338. Powell-Tuck J, Jamieson CP, Bettany GE, et al. A double blind, randomised, controlled trial of glutamine supplementation in parenteral nutrition. Gut. 1999;45:82-88.

339. Flaring UB, Rooyackers $\mathrm{OE}$, Wernerman J, Hammarqvist F. Glutamine attenuates post-traumatic glutathione depletion in human muscle. Clin Sci (Lond). 2003;104:275-282.

340. Goeters C, Wenn A, Merters N, et al. Parenteral L-alanyl-1-glutamine improves 0-month outcome in critically ill patients. Crit Care Med. 2002;30:2032-2037.

341. Peng X, Yan H, You Z, Wang P, Wang S. Effects of enteral supplementation with glutamine granules on intestinal mucosal barrier function in severe burned patients. Burns. 2004;30:135-139.

342. Umpleby AM, Carroll PV, Russell-Jones DL, Treacher DF, Jackson NC. Glutamine supplementation and GH/IGF-I treatment in critically ill patients: effects on glutamine metabolism and protein balance. Nutrition. 2002;18:127-129.

343. Ziegler TR, Ogden LG, Singleton KD, et al. Parenteral glutamine increases serum heat shock protein 70 in critically ill patients. Intensive Care Med. 2005;31:1079-1080.

344. Coëffier M, Claeyssens S, Hecketsweiler B, Lavoinne A, Ducrotté P, Dechelotte P. Enteral glutamine stimulates protein synthesis and decreases ubiquitin mRNA level in human gut mucosa. Am J Physiol. 2003:285:G260-G273.

345. Roig JC, Meetze WH, Auestad N, et al. Enteral glutamine supplementation for the very low birthweight infant: plasma amino acid concentrations. J Nutr: 1996;126:1115S-1120S.

346. Darmaun D, Roig JC, Auestad N, Sager BK, Neu J. Glutamine metabolism in very low birth weight infants. Pediatr Res. 1997;41:391-396.

347. Long CL, Nelson KM, DiRienzo DB, et al. Glutamine supplementation of enteral nutrition: impact on whole body protein kinetics and glucose metabolism in critically ill patients. JPEN J Parenter Enteral Nutr. 1995;19:470-476.

348. Sheridan RL, Prelack K, Yu YM, Lydon M, Petras L, Young VR. Short-term enteral glutamine does not enhance protein accretion in burned children: a stable isotope study. Surgery. 2004;135.

349. Houdijk APJ, Rijnsburger ER, Jansen J, et al. Randomised trial of glutamine-enriched enteral nutrition on infectious morbidity in patients with multiple trauma. Lancet. 1998;352:772-776.

350. Gore DC, Wolfe RR. Metabolic response of muscle to alanine, glutamine, and valine supplementation during severe illness. JPEN J Parenter Enteral Nutr. 2003;27:307-314. 
351. Darmaun D, Hayes V, Schaeffer D, Welch S, Mauras N. Effects of glutamine and recombinant human growth hormone on protein metabolism in prepubertal children with cystic fibrosis. J Clin Endocrinol Metab. 2004:80:1140.1152.

352. Quan ZF; Yang C. L. N, Li IL. Effect of glutamine on change in early postoperative intestinal permeability and its relation to systemic inflammatory response. World J Gastroenterol. 2004;10:1992-1994.

353. Kaibara A. Yoshida S. Yamasaki K, Ishibashi N. Kakegawa T. Effect of glutamine and chemotherapy on protein metabolism in tumorbearing rats. / Sint Res. 1994:57:143.149.

354. Naka S, Saito H, Hashiguchi Y. et al. Alanyl-giutamine-supplemented total parenteral nutrition improves survival and prutein metabolikm in rat protracted bacterial peritonitis model. JPENJ Parenter Enteral Nutr. 1000:20:417-423.

355. Holecek M, Skopec F, Skalska H. Sprongl L. Effect of alanyl-glutamine on leucine and protein metabrlism in endotoxemic rats. IPEN I Farenter Interal Nutr. 2000;24:215-222.

350. Holecek M. Skupec E, Sprongl I. Mraz I, Skalska H. Pecka M. Effect of alanyl-glutamine on leucine and protein metabulism in irradiated rats. Amino Acids. 2002;22:95-108.

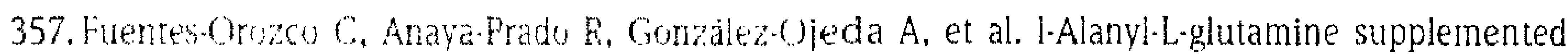
parentera! nutrition improves infectious morbidity in secondary peritonitis. Clin Nutr. 2004:23:13-21.

358. Walser M. Misinterpetation of nitrigent balances when glutamine stores fall or are replenished. Am J Clin Nutr. $1901: 53: 1337.1338$

350. Hiramatsu T. Cortiella I, Marchini IS, (Chapman TE, Young VR. Source and amount of dietary nonspecific nitrugen in relation to wholebody leucine, phenylalanine, and tyrosine kinetcis in young men. Am I Glin Nutr. 1404;54:1347-1355.

300. Unnebere K, Mialand M, Baltekrard L. Jenssen TG, Bjoro T, Revhaug A. Both growth hormone and exogenous glutamine increase gastruintestinal glutamine uptake in trauma. Ann Surg. 1997;225:97. 102.

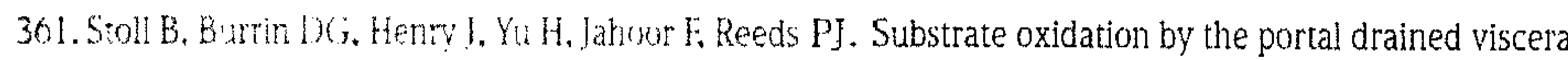
of fed piglete. An l flisiol. 1000:277:E168-E175.

362. Hankard $\mathrm{KG}$. Hammurd D. Haymond MW, Darmaun D. Oral glutamine slows down whole body frotein breakcown in lmcheme muscular dystrophy. Pediatr Res. 1998;43:222-220.

303. Tadros L.B. Willhofr NM. Taylor PM. Rennie MJ. Effects of glutamine deprivation on glutamine transpor and synthesis in primary culture of rat skeletal muscle. Am I Physiol 1993;205:E035.942.

304. Wat FW. Hundal HS. Downie S, Rennie M. Glutamine increases protein synthesis in heart, skeletal muscle, liver and git of dexamethasone-treated rats. Proc Nutr Soc. 1991:013.

365. Esser P. MCNurian MA. Wemerman 1. Vinnars E, Garlick PJ. Uncomplicated surgery, but not general anesthesia, dreceases muscle protein synthesis. Am J Physiol. 1992;262:E253-260.

3ho. Horel M. Wilians Ft. Iabbour K, Flakoll PJ. Chronic hypocaloric parenteral nutrition containing glutarnire promots nepatic rather than skeletal muscle or gut uptake of glutamine after fasting. JPEN

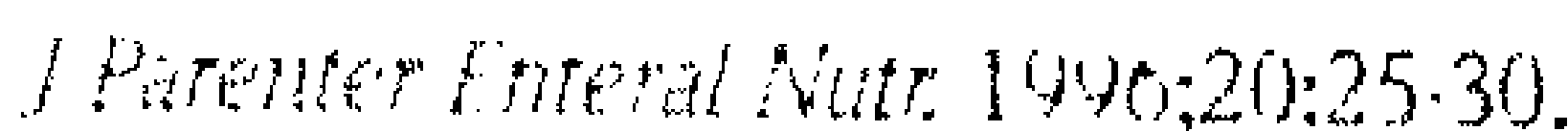

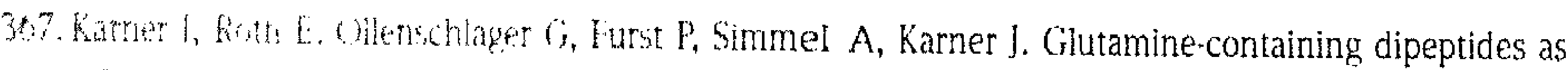

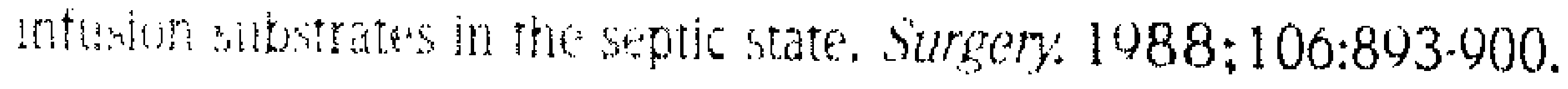

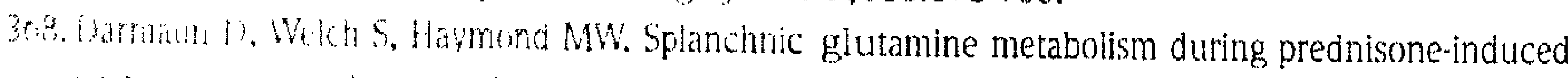

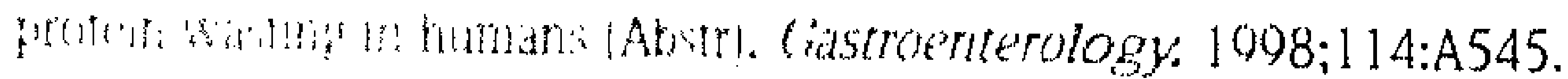

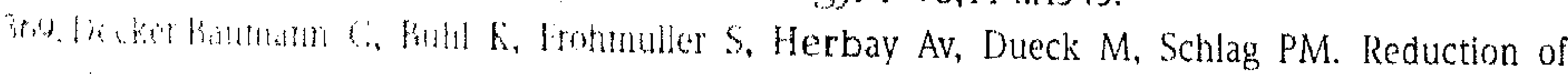

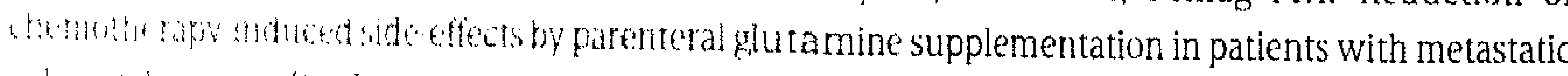

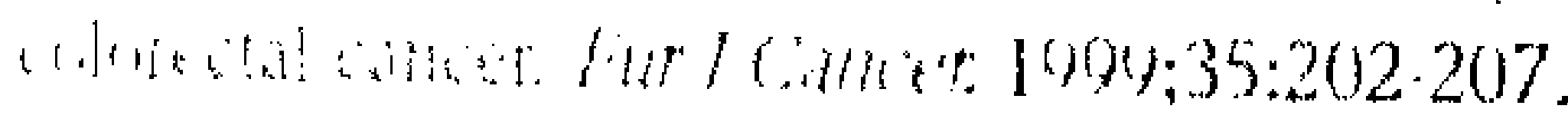


370. Kong SE, Hall JC, Cooper D, McCauley RD. Glutamine-enriched parenteral nutrition regulates the activity and expression of intestinal glutaminase. Biochim Biophys Acta. 2000;1475:67-75.

371. Klimberg VS, Souba WW, Sitren H, al. e. Glutamine enriched total parenteral nutrition supports gut metabolism. Surg Forum. 1989;40:0-8.

372. Dudrick PS, Austgen TR, Chen MK, al. e. Prefeeding with glutamine preserves intestinal gltuaminase activity in the endotxin treated rats (Abstr). J Parenter Enteral Nutr. 1992;16:19S.

373. Chen K, Nezu R, Sando K, et al. Influence of glutamine-supplemented parenteral nutrition on intestinal amino acid metabolism in rats after small bowel resection. Surg Today. 1996;26:618-623.

374. Chen K, Okuma T, ()kamura K, Torigoe Y, Miyauchi Y. Glutanine-supplemented parenteral nutrition improves gut mucosa integrity and function in endotoxemic rats. JPEN J Parenter Enteral Nutr. 1994;18:167-171.

375. Klimberg VS, Salloum RM, Kasper M, et al. Oral glutamine accelerates healing of the small intestine and improves outcome after whole abdominal radiation. Arch Surg. 1990;125:1040-1045.

376. Yoshida S, Leskiw MJ, Schluter MD, et al. Effect of total parenteral nutrition, systemic sepsis, and glutamine on gut mucosa in rats. Am J Physiol. 1992;263:E368-373.

377. Stein TP, Yoshida S, Schluter MD, Drews D, Assimon SA, Leskiw MJ. Comparison of intravenous nutrients on gut mucosal proteins synthesis. JPEN J Parenter Enteral Nutr: 1994;1 8:447-452.

378. Garcia-Arumi E, Schwartz S, Lopez-Hellin J, Arbos MA, Andreu AL, Farriol M. Addition of glutamine does not improve protein synthesis and jejunal mucosa morphology in non-hypercatabolic stress. Physiol Res. 1995;44:233-239.

379. Marchini JS, Nguyen P, Deschamps JY, Maugere P, Krempf $M$, Darmaun D. Effect of intravenous glutamine on duodenal mucosa protein synthesis in healthy growing dogs. Am J Physiol. $1999 ; 276:$ : 747.753.

380. van Acker BAC, Deutz NEP, Soeters PB. Increased intestinal protein synthesis during glutamineenriched enteral nutrition (Abstr). JPEN J Parenter Enteral Nutr: 1996;20:30.

381. Bouteloup-Demange C, Claeyssens S, Maillot C, Lavoinne A, Lerebours E, Dechelotte P. Effects of enteral glutamine on gut mucosal protein synthesis in healthy humans receiving glucocorticoids. Am I Physiol Gastrointest Liver Physiol. 2000;278:G677-681.

382. Mercier A, Eurin D, Poulet-Young V, Marret S, Dechelotte P. Effect of enteral supplementation with glutamine on mesenteric blood flow in premature neonates. Clin Nutr. 2003;22:133-137.

383. Karner J, Roth E. Influence of alanylglutamine infusion on gastrointestinal glutamine and alanine metabolism in anesthetized dogs. Metabolism. 1989;38:73-77.

384. Suzuki I, Matsumoto Y, Adjei AA, Shinjo S, Yamamoto S. Effect of a glutamine-supplemented diet on response to methicillin-resistant Staphylococcus aureus infection in mice. J Nutr Sci Vitaminol. 1993;39:405-410.

385. Liu C-T, Chen K-M, Chang P.L, Lii C.K. Glutamine utilization in activated lymphocytes from rats receiving endotoxin. J Surg Res. $2001 ; 96: 240-254$.

380. Thebault $S$, Deniel N, Marion R, et al. Proteomic analysis of glutamine-treated human intestinal epithelial HCT-8 cells under basal and inflammatory conditions. Proteomics. 2006;0:3926-3937. 


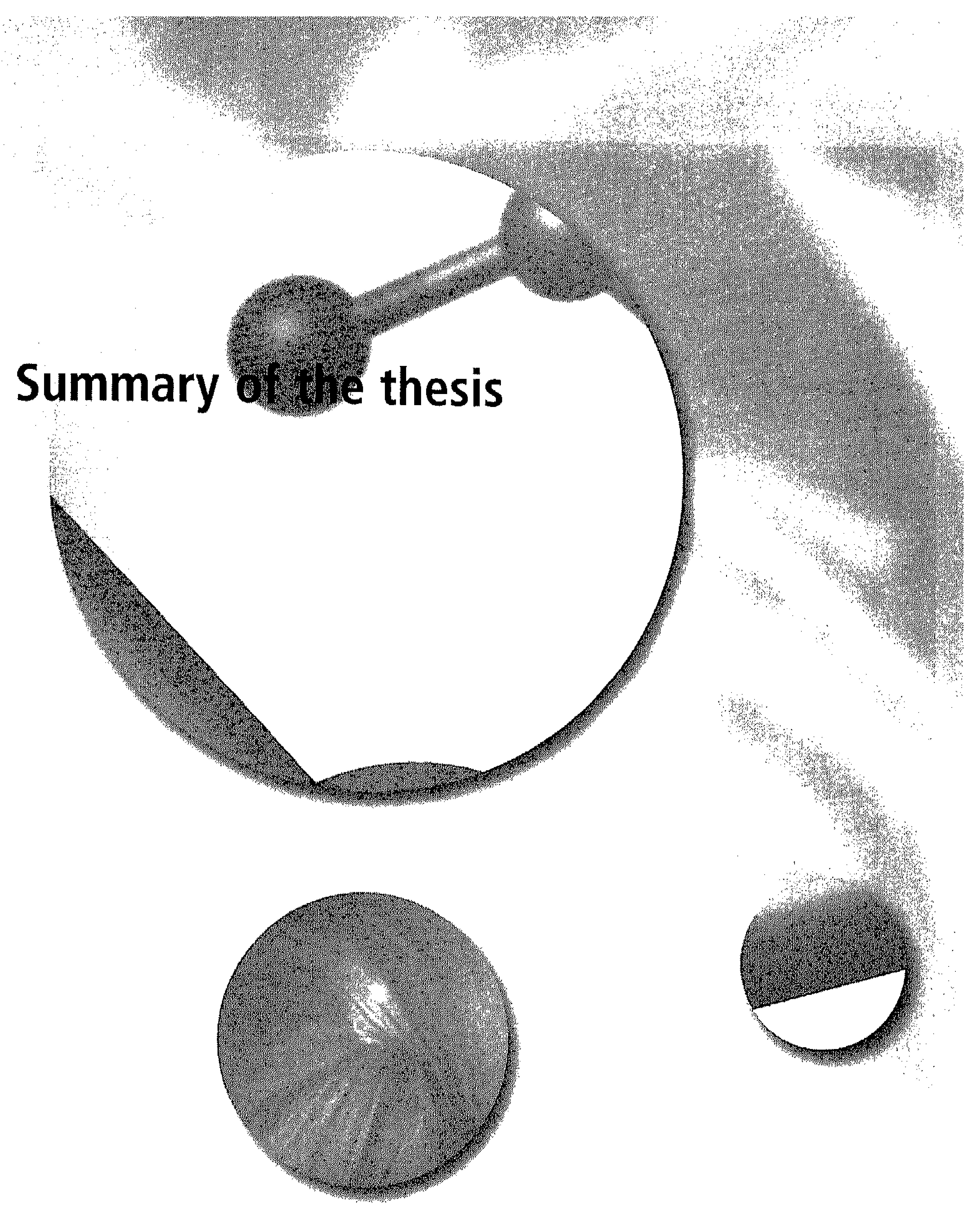




\section{SUMMARY AND CONCLUSIONS}

In the altered amino acid metabolism after (surgical) trauma or in critical illness glutamine plays a central role. Not only is glutamine at the crossroads of diverse metabolic pathways but under stressed conditions it is quantitatively the most important nitrogen containing molecule released by muscle tissue. Furthermore, a large decrease in plasma and muscle glutamine concentrations, correlating with the degree of catabolism, suggests that endogenous glutamine may become deficient under certain conditions, such as during severe stress, or in the presence of diminished muscle cell mass. Indeed, glutamine supplementation in various circumstances has resulted in metabolic but also clinically significant effects. These results, however, were not always concordant leading to discussion about its usefulness in clinical practice. A major problem is that in contrast to pharmaceuticals, glutamine is produced and consumed endogenously. Exogenous glutamine may affect its endogenous metabolism. Finally the route and form in which glutamine is administered differ in various studies compromising comparability.

This thesis consists of three major sections. After a brief introduction and explanation of the underlying hypotheses of this thesis, the first part describes the effects of depletion, (surgical) trauma and critical illness on glutamine metabolism in a pig model and in patients. In part 2 we describe the results of 3 studies investigating the effects of glutamine supplementation on endogenous glutamine metabolism and on gut mucosal metabolism, function and structure. In the last section a review of the literature including the results of the studies described in the first 2 parts is presented. After a summary of biochemical aspects of glutamine and methodologies used to study interorgan metabolism (including stable isotopes and AV balance techniques), (inter)organ metabolism in healthy and metabolically stressed organisms is reviewed followed by the effects of glutamine supplementation on endogenous glutamine metabolism.

In chapter 1 the background leading to the studies presented in this thesis is summarized. Then, after a brief review of the chemical and physiological properties of glutamine, the methodologies concerning techniques used in the studies presented in this thesis are explained.

In Chapters 2-6 we investigated effects of several types of stress on glutamine metabolism. In chapter 2 the results are reported of a study employing stable isotopes to determine glutamine flux in plasma in surgical patients. Despite confirmation of decreased glutamine levels in plasma and muscle tissue, as had been observed earlier, no change in glutamine Ra was demonstrated. This might have been due to differences in protein turnover, changes in glutamine production by other organs, or possibly due to an erroneous outcome as a consequence of a methodological problem as discussed in the first part of the discussion. In chapter 3 interorgan metabolism after surgical stress is described in more detail in a porcine model using both arteriovenous balance and isotopic techniques. On the $2^{\text {nd }}$ day after laparotomy, a large efflux of glutamine was observed which could at least partly be explained by increased protein breakdown rates. Uptake of glutamine by the PDV decreased after surgery, which was associated with a decrease in glutamine disappearance 
rates and an increased dependency on glutamine de novo synthesis. The liver switched from net glutamine production to significant glutamine uptake. The hepatic glutamine oxidation rates increased and relative glutamine synthesis rates decreased. Finally, the kidney appeared not to play a significant role in glutamine interorgan metabolism in the postoperative pig model. In chapter 4 the effects of surgery on gut mucosal glutathione metabolism are described in more detail. Effects of glutamine on mucosal glutathione metabolism may be one of the possible mechanisms through which glutamine sorts its effects. Compared to control values we found significant decreases in gut mucosal glutamine concentrations, but no significant changes in mucosal glutathione concentrations. Absolute glutathione synthesis rates, however, decreased with $38 \%$. Although significant correlations between glutathione concentrations and glutamine and glutamate concentrations were found, these were not specific since several other amino acids showed similar correlations. In steady state conditions these imply that loss of reduced glutathione either by oxidation or export out of the cell is decreased in the postoperative period. Whether this might have detrimental effects remains to be determined. In chapter 5 the role of the lungs in glutamine metabolism in septic patients was studied. Using arteriovenous sampling across the lungs the results in septic patients were compared with data obtained from patients before undergoing coronary bypass surgery. We found that during sepsis a considerable amount of glutamine is furnished by the lungs in contrast to the control population. It was estimated that at least half of this glutamine results from protein breakdown. Severity of illness had no impact on glutamine fluxes in contrast to the presence of pulmonary infiltrates on chest X-rays: in the presence of pulmonary inflammation, glutamine efflux does not differ from zero. This may indicate local glutamine consumption, possibly by activated immune cells. In chapter 6 the effects of nutritional depletion versus inflammatory stress on glutamine metabolism are examined. In this study we determined the relation of (anthropometric) measures of depletion and blood parameters indicating inflammatory activity with glutamine concentrations in plasma and duodenal mucosa. Furthermore relations with mucosal morphology and intestinal permeability were also studied. Inflammation but not depletion significantly affected plasma and tissue glutamine concentrations and also gut barrier function. On the other hand, depletion but not inflammation was associated with diminished villus height.

In the next section we describe the results of 4 studies focusing on the effects of exogenous glutamine supplementation. In chapter 7 we describe the functional results of a prospective randomised study of glutamine supplemented total parenteral nutrition in a nutritionally depleted patient population. Despite previous clues that depletion is a risk factor for glutamine depletion, no significant changes in glutamine concentrations were observed. Moreover, no effects on intestinal permeability, mucosal morphology or gut mucosal inflammation were found. Analysis of differences between patient populations in this study and a previously published report by our group, suggested that possibly not depletion but rather systemic inflammation may have an important effect on glutamine metabolism. This is confirmed by findings presented in chapter 6 . In chapter 8 the effects of glutamine enriched parenteral nutrition on whole body glutamine fluxes in patients are reported. The most important finding was that glutamine endogenous rate of appearance 
is not diminished thereby increasing glutamine availability for consumption. In chapter $\mathbf{9}$, this is studied in more detail in pigs. Pigs received TPN either with or without glutamine in the absence of a catabolic insult. Infusion of TPN increased whole body protein turnover and had anabolic effects on muscle and intestinal tissue. Addition of glutamine to TPN results in enhanced protein deposition in the muscle. Protein synthesis in the gut decreased, but the relative decrease in proteolysis was somewhat larger. Despite increased arterial plasma glutamine concentrations, glutamine enriched TPN did not change muscle glutamine release. Furthermore, it did not increase but rather decreased gut glutamine consumption and mucosal glutamine concentrations in the presence of a lower (although statistically not significant) extraction rate. This was accompanied by an increased de novo glutamine synthesis in the PDV. The supplemented glutamine was not metabolized by the gut, but rather processed by the liver.

In chapter 10 the findings presented in the preceding chapters are discussed in a broader context using data available in the literature. First, glutamine metabolism and methodological issues concerning measurements of (inter)organ metabolism are discussed. Subsequently data regarding glutamine metabolism in healthy and metabolically stressed organisms are reviewed. Finally the effects of glutamine supplementation on endogenous glutamine metabolism are discussed. 


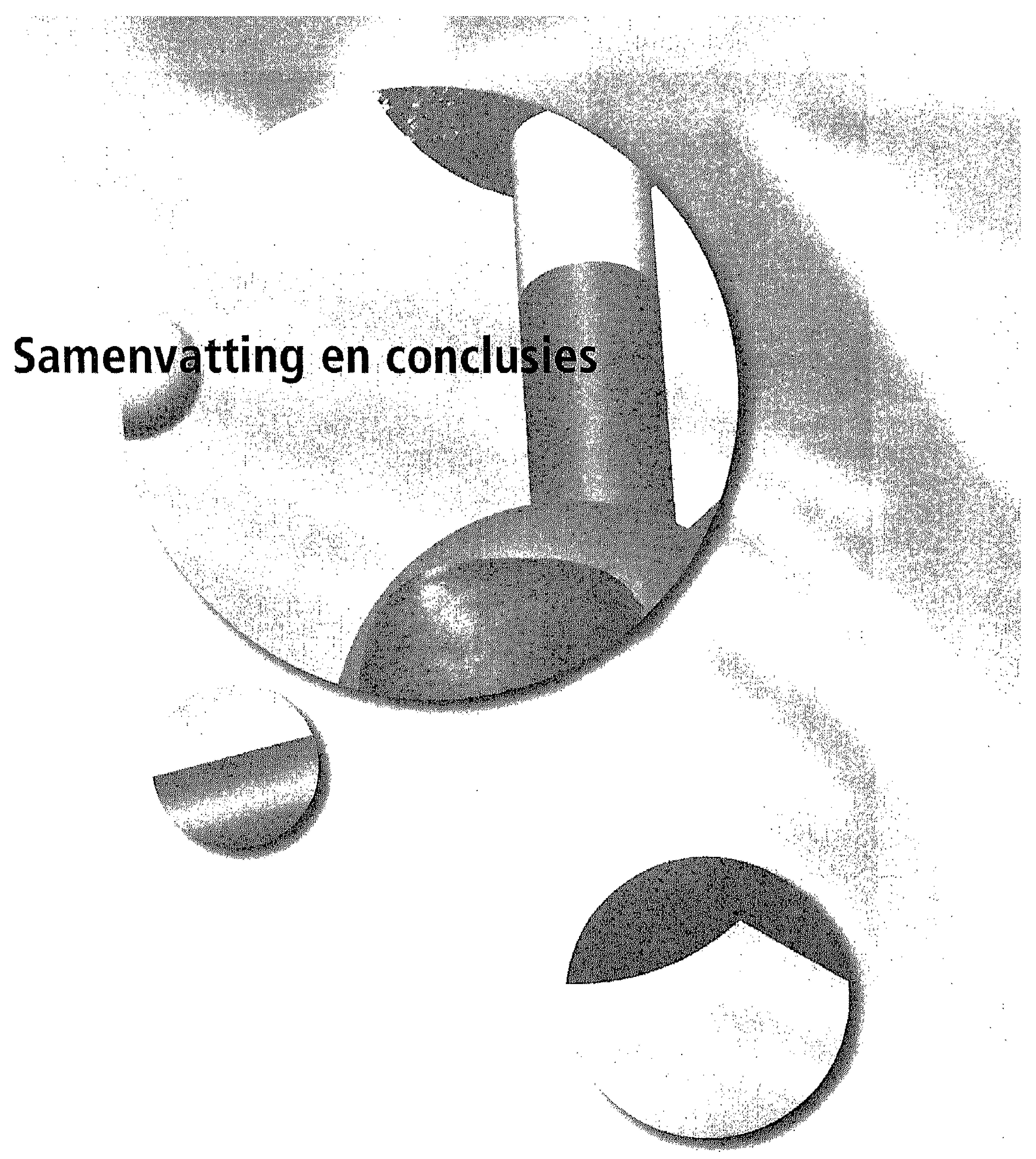




\section{SAMENVATTING EN CONCLUSIES}

Glutamine speelt een centrale rol in het veranderde aminozuur metabolisme na (chirurgisch) trauma of bij de ernstig zieke patiënt. Glutamine speelt een centrale rol in verscheidene metabole processen maar tevens is glutamine de belangrijkste "stikstofdrager" voor transport van stikstof vanuit het spierweefsel naar andere organen tijdens metabole stress. Omdat er een relatie bestaat tussen katabolie en (daling van) plasma en spier glutamine concentraties, werd de hypothese geformuleerd dat endogene glutamine productie insufficiënt kan worden onder bepaalde condities zoals gedurende ernstige (en langdurige) ziekte of indien er sprake is van een geringe spiermassa. Uit verschillende studies blijkt inderdaad dat glutamine toediening metabole maar ook klinische effecten heeft. Echter, niet alle studies hebben dezelfde resultaten zodat er nog geen volledige overeenstemming bestaat over het nut van toepassing in de praktijk. Een van de problemen is dat in tegenstelling tot farmaceutische middelen, glutamine ook endogeen geproduceerd wordt. Exogeen toegediend glutamine zou de endogene productie kunnen beïnvloeden. Daarnaast blijkt de wijze en vorm van toediening duidelijk te verschillen tussen de verschillende studies waardoor de uitkomsten moeilijk te vergelijken zijn.

Dit proefschrift is onderverdeeld in drie delen. $\mathrm{Na}$ de introductie waarbij ook de hypothese die ten grondslag ligt aan dit proefschrift wordt toegelicht, worden in het eerste deel de resultaten besproken van een aantal studies (dierexperimentele en klinische studies) naar de effecten van depletie, (chirurgisch) trauma en ernstige ziekte op glutamine metabolisme. In het $2^{e}$ deel worden 3 studies beschreven die de effecten van glutamine toediening op het endogene glutamine metabolisme en metabole, functionele en structurele aspecten van de darm als onderwerp hadden. In het laatste deel wordt een uitgebreid literatuur overzicht over glutamine metabolisme beschreven waarbij de data van het $1 \mathrm{e}$ en $2^{e}$ deel worden geïncorporeerd. In dit review worden de biochemische aspecten van glutamine en methodologische aspecten van interorgaan metabolisme studies besproken, waaronder gebruik van stabiele isotopen en arterioveneuze balans studies. Vervolgens wordt (inter)orgaan glutamine metabolisme bij gezonde en metabool gestreste organismen behandeld. Tot slot worden ook de effecten van glutamine suppletie op het endogene metabolisme besproken.

In hoofdstuk 1 worden de achtergronden leidend tot dit proefschrift besproken. Vervolgens worden de chemische en fysiologische aspecten van glutamine kort samengevat, waarna de meetmethodieken die in dit proefschrift worden gehanteerd worden toegelicht.

In de hoofdstukken 2.6 bespreken we de effecten van verscheidene typen pathofysiologische stress op het glutamine metabolisme. In hoofdstuk 2 worden de resultaten gepresenteerd van een studie waarbij met stabiele isotopen effecten van chirurgisch trauma op glutamine flux is gemeten. Chirurgisch trauma leidde tot verlaging van glutamine concentraties in plasma en spier, maar glutamine Ra bleef onveranderd. Mogelijke verklaringen hiervoor zijn veranderingen in eiwitmetabolisme, veranderingen in glutamine productie door andere organen dan spier, of wellicht het gevolg van een methodologisch probleem bij de meting van glutamine flux zoals besproken in het eerste 
deel van de discussie. In hoofdstuk 3 wordt het interorgaan metabolisme van glutamine na chirurgische stress nader beschreven aan de hand van experimenten in een varkensmodel waarbij een combinatie van stabiele isotopen en arterioveneuze balans technieken is gebruikt. Op de $2^{\text {e }}$ postoperatieve dag na laparotomie was er sprake van een grote uitvloed van glutamine uit de spier die deels verklaarbaar was door verhoogde eiwitafbraak. Opname van glutamine door de darm nam af net zoals het glutamine verbruik. Er was sprake van een toegenomen glutamine productie door de darm. In plaats van glutamine te produceren, nam de lever na operatie glutamine op. De relatieve bijdragen van de novo synthese nam af en glutamine oxidatie nam toe. De nier bleek uiteindelijk niet veel bij te dragen aan interorgaan glutamine metabolisme in dit model. In hoofdstuk 4 wordt ingezoomd op de effecten van chirurgisch trauma op glutathion metabolisme in het darmslijmvlies. De reden hiervoor is dat de effecten van glutamine mogelijk deels verklaard kunnen worden door veranderingen in het glutathion metabolisme. Na chirurgie vonden we wel een significante daling van glutamine concentraties in het darmslijmvlies maar geen significante veranderingen in glutathion concentraties. Echter, absolute glutathion synthese snelheden namen af met $38 \%$. Ondanks significante correlaties tussen glutamine en glutamaat concentraties met glutathion concentraties waren deze niet specifiek omdat er ook vergelijkbare significante relaties bestonden met andere aminozuren. Onder voorwaarde van steady state, betekent deze bevinding dat in de postoperatieve fase verlies van gereduceerd glutathion (via oxidatie of uitscheiding door de cell moet zijn afgenomen. Of dit klinische relevantie heeft is onduidelijk.

In hoofdstuk 5 is de rol van de longen in het interorgaan metabolisme van glutamine bestudeerd in septische patiënten. Door respectievelijk in de a. pulmonalis en systemisch arterieel in de a. radialis te meten, kon de arterioveneuze balans over de longen worden berekend. De populatie van septische patiënten werd vergeleken met een groep patiënten die een open hart operatie zouden ondergaan. Uit deze studie bleek dat de longen tijdens sepsis een significante hoeveelheid glutamine produceren, in vergelijking met opname van glutamine door de longen in de controle groep. Op basis van de gegevens van deze studie werd geschat dat ongeveer de helft van de glutamine afkomstig was van eiwitafbraak. De ernst van sepsis had geen effect op glutamine fluxen, in tegenstelling tot de aan- of afwezigheid van longinfiltraten op de thorax foto: bij aanwezigheid van een infiltraat was er geen sprake meer van glutamine efflux. Dit zou kunnen wijzen op toegenomen lokale glutamine consumptie bijvoorbeeld door geactiveerde immuuncellen. In hoofdstuk 6 worden de relaties tussen (anthropometrische) maten voor depletie en klinisch chemische dan wel hematologische maten voor ontstekingsactiviteiten met glutamine concentraties in plasma en darmslijmvlies bekeken. Tevens worden relaties met slijmvliesmorfologie en darm barrière functie bestudeerd. Het bleek dat de aanwezigheid van ontstekingsactiviteit maar niet van depletie significant gerelateerd was met glutamine concentraties in darm en plasma, en ook met darmpermeabiliteit. Aan de andere kant was depletie maar niet ontstekingsactiviteit gerelateerd aan veranderingen in darmmorfologie.

In het volgende deel beschrijven we het resultaat van 3 studies die gericht zijn op de effecten van exogene glutamine suppletie. In hoofdstuk 7 worden de functionele effecten gepresenteerd van een prospectieve gerandomiseerde studie naar glutamine verrijkte totale 
parenterale voeding in een deplete patiëntenpopulatie. Ondanks eerdere aanwijzingen dat depletie een risicofactor is voor glutamine depletie, werden geen effecten in glutamine concentraties in plasma gevonden. Ook werden geen effecten op darmpermeabiliteit. darmslijmvlies morfologie of tekenen van toegenomen inflammatoire activiteit in de darm gevonden. Op basis van analyse van de verschillen in patiëntenpopulatie tussen deze en een eerder door de onderzoeksgroep gerapporteerde studie naar de effecten van glutamine verrijkte totale parenterale voeding, werd de hypothese gesteld dat niet zozeer depletie maar veeleer aanwezigheid van systemische ontstekingsactiviteit effect heeft op glutamine metabolisme. Dit werd bevestigd door data die zijn beschreven in hoofdstuk 6 . In hoofdstuk 8 worden de effecten van toediening van glutamine verrijkte totale parenterale voeding op de glutamine flux in plasma bij patiënten beschreven. De belangrijkste bevinding van deze studie was dat de endogene glutamine flux niet afnam door toediening van extra glutamine, waardoor dit resulteerde in een toename van het glutamine aanbod.

In hoofdstuk 9 zijn de metabole effecten van infusie van glutamine verrijkte totale parenterale voeding in meer detail bestudeerd in een varkensmodel. De varkens werden gerandomiseerd tussen 2 groepen die totale parenterale voeding toegediend kregen waarbij de ene groep wel en de andere groep geen glutamine kreeg. Infusie van parenterale voeding resulteerde in een toegenomen turnover van eiwit op totaal lichaamsniveau, en had anabole effecten op spier en darmweefsel. Door toevoeging van glutamine nam eiwitdepositie in de spier verder toe. Aan de andere kant nam eiwitsynthese in de darm af, maar door een sterkere stijging van eiwitafbraak was het netto effect ook positief. Glutamine toediening leidde tot verhoogde plasma concentraties maar niet tot veranderingen in glutamine efflux uit de spier. De opname van glutamine door de darm werd door deze verhoogde concentraties ook niet versterkt, maar nam juist af. Ook mucosa glutamine concentraties daalden. Tegelijkertijd nam de de novo synthese van glutamine door de darm toe.

Het extra toegediende glutamine werd derhalve niet door de darm maar voornamelijk door de lever gemetaboliseerd.

In hoofdstuk 10 worden de hierboven beschreven resultaten in een breder kader geplaatst door ze te integreren in een literatuuroverzicht betreffende glutamine metabolisme. In het eerste deel worden enkele basale aspecten van glutamine metabolisme toegelicht waarbij wat dieper wordt ingegaan op methodologische aspecten van metingen van interorgaan metabolisme. Daarna worden achtereenvolgens de beschikbare gegevens over glutamine metabolisme in gezondheid, tijdens ziekte en bij toediening van exogeen glutamine samengevat. 


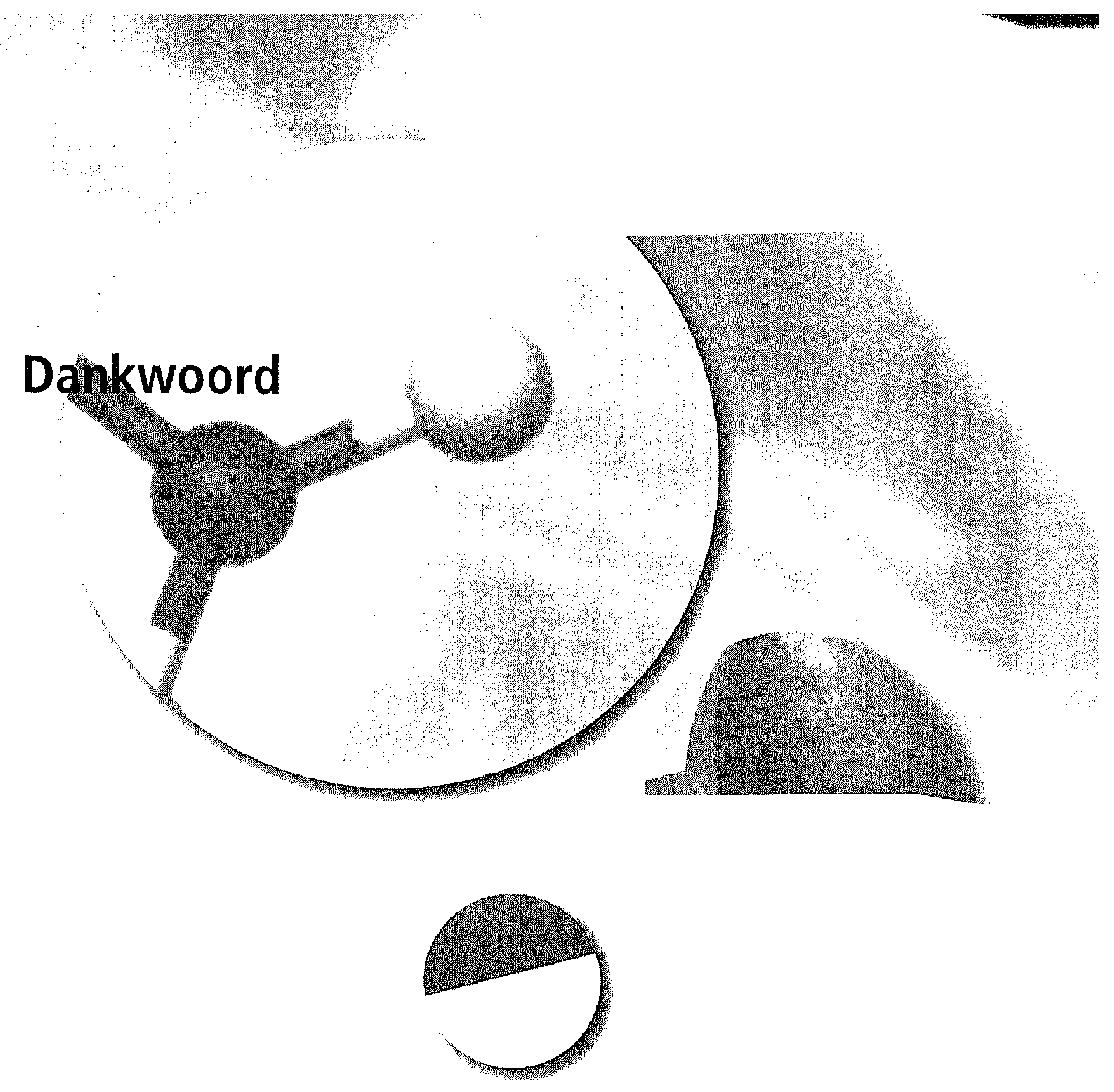




\section{DANKWOORD}

"No one who achieves success does so without acknowledging the help of others. The wise and confident acknowledge this help with gratitude" - Alfred North Whitehead

Bij een promotie aan een universiteit wordt de doctorsgraad verkregen. Dit is de hoogste academische graad. In vroegere tijden werd dikwijls op stellingen gepromoveerd. Echter, sinds 1877 wordt hiervoor een proefschrift geschreven dat in het openbaar verdedigd wordt. Dat is iets wat je niet alleen doet maar waarbij je van velen afhankelijk bent. En dat zo'n proces niet altijd gladjes verloopt, zal ook duidelijk zijn. Na doormaken van vele hoogte- maar ook dieptepunten is het einde van het promotietraject bereikt.

Ik ben hiervoor velen dank verschuldigd maar in de eerste plaats de patiënten en hun families die ondanks de moeilijke situatie waarin zij verkeerden, belangeloos aan de verschillende studies hebben meegewerkt. De leden van het voedingsteam, de apotheek van het AZM, betrokken diëtistes en verpleegkundigen van de verschillende afdelingen dank ik voor de praktische ondersteuning bij de verschillende onderzoeken. Dit geldt eveneens voor de gastroenterologen en medewerkers van de scopieafdeling. Verder zou dit proefschrift nooit tot stand zijn gekomen zonder hulp van de medewerkers van de verschillende laboratoria (het metabole lab, het SIRC, het pathologisch-anatomisch lab, het klinisch-chemisch lab en het farmacologisch lab) en het CPV waar ik veel heb geleerd en waar veel van de data van dit proefschrift vandaan komen. De wetenschappelijke artikelen waaruit dit proefschrift is opgebouwd, konden enkel tot stand komen met hulp van de kritische inbreng van de co-auteurs waarvoor veel dank. Ook de collegae promovendi wil ik bedanken voor de gezelligheid maar ook fysieke en mentale ondersteuning. Mijn promotores Peter Soeters en Maarten von Meyenfeldt hebben mij in staat gesteld om de beschreven studies uit te voeren. Maar naast hun wetenschappelijke en linguiistische kennis van het Engels, heb ik ook op chirurgisch en niet medische terreinen veel van hen geleerd. Peter en Maarten, hartelijk dank voor alle tijd die jullie in mij en dit proefschrift hebben willen steken en voor het gegeven vertrouwen.

Aan het eind van het schrijven van een proefschrift ben je ten slotte ook nog afhankelijk van anderen voor de promotie zelf. De leden van de beoordelingscommissie, prof. dr. R.W. Stockbrügger, prof. dr. M.J.H.M. van Dieijen - Visser, prof. dr. P.J.E.H.M. Kitslaar, prof. dr. P.A.M. van Leeuwen en prof. dr. K.M.L. Leunissen dank ik voor hun bereidheid het manuscript te beoordelen en zitting te nemen in de beoordelingscommissie. Ton Hoofwijk dank ik voor het kritisch doornemen van het proefschrift. Michiel Heeren dank ik dat hij de rol van paranimf op zich heeft willen nemen. Met Bernadette van Acker heb ik de meeste humane experimenten van dit proefschrift uitgevoerd. Ik heb veel geprofiteerd van haar gedrevenheid, accuratesse en niet aflatende bereidheid om me met raad en daad bij te staan. Ik ben dankbaar dat zij als paranimf bij de promotie aanwezig wil zijn.

Mijn vader was altijd zeer betrokken bij de voortgang van het onderzoek, maar kan de promotie zelf helaas niet meer meemaken. Zowel hem als mijn moeder dank ik voor hun niet aflatende steun en vertrouwen en aan hen is dit proefschrift opgedragen.

Vanaf 1815 kon men bij de faculteit geneeskunde na het behalen van de graad Medicinae Doctor (MD) nogmaals promoveren in de chirurgie. Riquette, Hannah, Willem, Julia en Pieter: gelukkig kan dat tegenwoordig niet meer. 



\section{CURRICULUM VITAE}

Karel Hulsewe was born on June 5th 1968 in Velp, The Netherlands. He attended secondary school in Arnhem (Stedelijk Gymnasium) and later in Venlo (Marianum College). After his graduation in 1986, he studied Medicine at Maastricht University, Maastricht, The Netherlands. He graduated from Medical School in 1993 and subsequently entered military service during which he was detached to the Multinational Force and Observers, El Gorah, Sinai, Egypt where he worked in a multinational clinic. In 1994 he started his surgical training which was combined with a research post (AGIKO) at the department of Surgery, Academic Hospital Maastricht (Head Prof. dr. G. Kootstra). In 1995 he received an NWO fellowship (AGIKO stipendium). His surgical training continued at the department of Surgery of the Maasland Ziekenhuis, Sittard (Head dr. A.G.M. Hoofwijk). After graduation in November 2003, he worked as a clinical fellow at the department of hepatobiliary surgery, University Hospital Bellvitge, Barcelona, Spain. Since October 2004 he is a member of the staff of the department of Surgery, Maaslandziekenhuis, Sittard, The Netherlands.

He is married to Riquette Evers, with whom he has 4 children: Hannah, Willem, Julia and Pieter. 